\title{
VISUALISING THE CHARGE AND COOPER PAIR DENSITY WAVES IN CUPRATES
}

\author{
Stephen Edkins
}

A Thesis Submitted for the Degree of PhD at the University of St Andrews

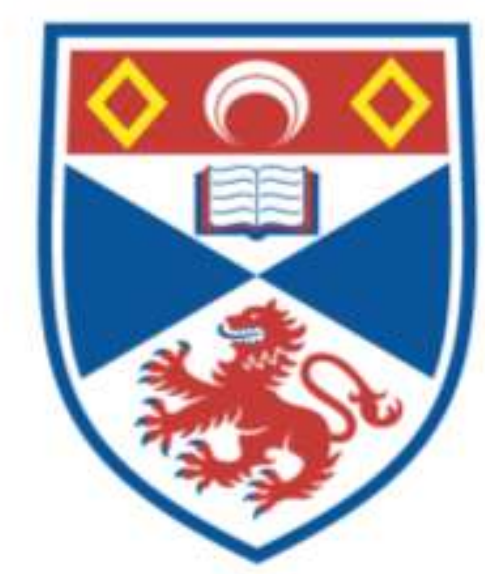

2016

Full metadata for this item is available in

St Andrews Research Repository

at:

http://research-repository.st-andrews.ac.uk/

Please use this identifier to cite or link to this item: http://hdl.handle.net/10023/9888

This item is protected by original copyright 


\title{
Visualising the Charge and Cooper Pair Density Waves in Cuprates
}

\author{
Stephen Edkins
}

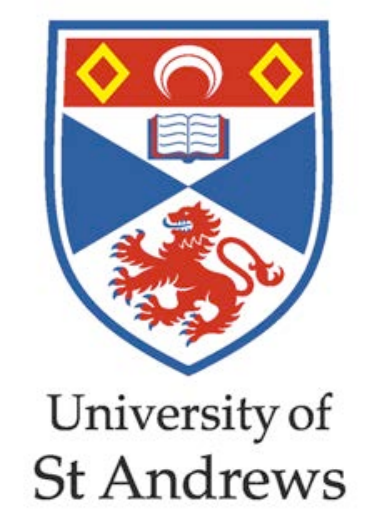

This thesis is submitted in partial fulfilment for the degree of $\mathrm{PhD}$ at the

University of St Andrews

October 2016 



\section{Candidate's declarations:}

I, Stephen David Edkins, hereby certify that this thesis, which is approximately 40,000 words in length, has been written by me, and that it is the record of work carried out by me, or principally by myself in collaboration with others as acknowledged, and that it has not been submitted in any previous application for a higher degree.

I was admitted as a research student in August, 2012 and as a candidate for the degree of Doctor of Philosophy in August, 2012; the higher study for which this is a record was carried out in the University of St Andrews between 2012 and 2016.

Date: $8 / 9 / 2016$

Signature of candidate

\section{Supervisor's declaration:}

I hereby certify that the candidate has fulfilled the conditions of the Resolution and Regulations appropriate for the degree of Doctor of Philosophy in the University of St Andrews and that the candidate is qualified to submit this thesis in application for that degree.

Date: $8 / 9 / 2016$

Signature of supervisor

\section{Permission for publication:}

In submitting this thesis to the University of St Andrews I understand that I am giving permission for it to be made available for use in accordance with the regulations of the University Library for the time being in force, subject to any copyright vested in the work not being affected thereby. I also understand that the title and the abstract will be published, and that a copy of the work may be made and supplied to any bona fide library or research worker, that my thesis will be electronically accessible for personal or research use unless exempt by award of an embargo as requested below, and that the library has the right to migrate my thesis into new electronic forms as required to ensure continued access to the thesis. I have obtained any third-party copyright permissions that may be required in order to allow such access and migration, or have requested the appropriate embargo below.

The following is an agreed request by candidate and supervisor regarding the publication of this thesis:

\section{PRINTED COPY}

No embargo on print copy

\section{ELECTRONIC COPY}

No embargo on electronic copy

Date: $8 / 9 / 2016$

Signature of candidate 



\section{Abstract}

The study of cuprate high-temperature superconductors has undergone a recent resurgence due to the discovery of charge order in several families of cuprate materials. While its existence is now well established, little is known about its microscopic origins or its relationship to high-temperature superconductivity and the pseudogap. The aim of the research presented in this thesis is to address these questions.

In this thesis I will report on the use of spectroscopic-imaging scanning tunnelling microscopy (SI-STM) to visualise the short-ranged charge density wave (CDW) in $\mathrm{Bi}_{2} \mathrm{Sr}_{2} \mathrm{CaCu}_{2} \mathrm{O}_{8+\delta}$ and $\mathrm{Na}_{x} \mathrm{Ca}_{2-x} \mathrm{CuO}_{2} \mathrm{Cl}_{2}$. Building on previous measurements of the intra unit-cell electronic structure of cuprates, I introduce sub-lattice segregated SISTM to individually address the atomic sub-lattices in the $\mathrm{CuO}_{2}$ plane with spatial phase sensitivity. Using this technique I establish that the CDW in $\mathrm{Bi}_{2} \mathrm{Sr}_{2} \mathrm{CaCu}_{2} \mathrm{O}_{8+\delta}$ and $\mathrm{Na}_{x} \mathrm{Ca}_{2-x} \mathrm{CuO}_{2} \mathrm{Cl}_{2}$ has a previously unobserved $d$-symmetry form factor, where a breaking of rotational symmetry within the unit cell is modulated periodically in space.

Towards identifying a mechanism of CDW formation, I establish that the amplitude of CDW modulations in the electronic structure are maximal at the pseudogap energyscale and that these modulations exhibit a spatial phase difference of $\pi$ between filled and empty states. Together with the doping evolution of the CDW wave-vector this highlights the role of the low-energy electronic structure of the pseudogap regime in CDW formation.

To elucidate the relationship between the CDW and the superconducting condensate I will introduce nanometer resolution scanned Josephson tunnelling microscopy (SJTM). In this approach the Cooper pair (Josephson) tunnelling current between a $\mathrm{Bi}_{2} \mathrm{Sr}_{2} \mathrm{CaCu}_{2} \mathrm{O}_{8+\delta}$ sample and a scan-able $\mathrm{Bi}_{2} \mathrm{Sr}_{2} \mathrm{CaCu}_{2} \mathrm{O}_{8+\delta}$ nano-flake STM tip is used to directly visualise the superconducting condensate. I will report the observation of a periodic modulation in the Cooper pair condensate at the same wave-vector as the CDW, the first direct detection of a periodically modulating condensate in any superconductor. 



\section{Acknowledgements}

I would first and foremost like to thank my supervisors Séamus Davis and Andy Mackenzie for their support and guidance, as well as teaching me a great deal. I have benefited greatly from the research environment in both of their groups, of which membership has been a privilege.

The research presented in chapter 4 of this thesis was carried out in close collaboration with Mohammad Hamidian and Kazuhiro Fujita to whom I extend my gratitude for sharing their expertise in spectroscopic-imaging STM. The research presented in chapters 5 and 7 was conducted in collaboration with Mohammad Hamidian and Andrey Kostin. I thank Mohammad for his tutelage in the operation of a cryogenic STM.

The idea of examining the difference between the oxygen sub-lattices of the $\mathrm{CuO}_{2}$ plane in SI-STM spectroscopic maps to reveal a $d$-symmetry form factor CDW was originally suggested by Subir Sachdev. I would like to thank him for sharing this with us as well as for useful discussions. I would also like to thank Andrey Chubukov for his very useful comments in the preparation of chapter 6 .

Finally, I would like to thank all of my colleagues past and present for their help, advice and friendship, as well as my family for their love and support.

I acknowledge studentship funding from the EPSRC via the Scottish Condensed Matter Center for Doctoral Training under grant number EP/G03673X/1. 



\section{Contents}

$\begin{array}{lll}\text { Declaration } & \text { iii }\end{array}$

$\begin{array}{ll}\text { Abstract } & \mathbf{v}\end{array}$

Acknowledgements vii

List of Figures $\quad$ xiii

List of Tables $\quad$ xvii

1 Unconventional Superconductivity and Density Wave Order in Cuprates 1

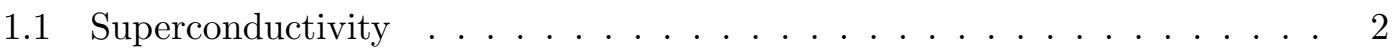

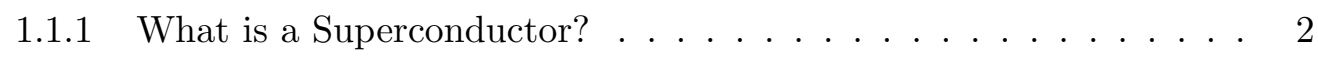

1.1.2 BCS Theory of Superconductivity _. . . . . . . . . . . 5

1.2 High-Temperature Unconventional Superconductivity in Cuprates . . . . . 10

1.2.1 Effective Models of the $\mathrm{CuO}_{2}$ Plane . . . . . . . . . . . . . 12

1.2 .2 Phase Diagram . . . . . . . . . . . . . . . . . . . . . . . . . . . . . . . . 13

1.2 .3 Anti-Ferromagnetism . . . . . . . . . . . . . . . . 14

1.2.4 High-Temperature $d$-Wave Superconductivity . . . . . . . . . . . . 14

1.2 .5 Strange Metal . . . . . . . . . . . . . . . . . . . . 15

1.2 .6 The Pseudogap . . . . . . . . . . . . . . . . . . 16

1.3 Charge Density Waves . . . . . . . . . . . . . . . . . 17

1.4 Unconventional Density Waves in Cuprates . . . . . . . . . . . . . . . . 19

1.5 The Significance of CDW in Cuprates . . . . . . . . . . . . . 21

1.6 Organisation of this Thesis . . . . . . . . . . . . . . 23

2 Spectroscopic-Imaging STM (SI-STM) $\quad 25$

2.1 Quantum Tunnelling as a Spectroscopy . . . . . . . . . . . . . . . . 26

2.1.1 Tunnelling Hamiltonian Formalism . . . . . . . . . . . . . . . 26

2.1.2 Normal - Insulator - Normal (NIN) Tunnelling . . . . . . . . . . . 27

2.1.3 Superconductor-Insulator-Normal (SIN) Tunnelling . . . . . . . . . 29

2.1.4 Superconductor-Insulator-Superconductor (SIS) Tunnelling . . . . 32

2.2 STM - Principles . . . . . . . . . . . . . . . . . . . 32

2.3 STM - Modes of Operation . . . . . . . . . . . . . . . 34

2.3 .1 Topography . . . . . . . . . . . . . . . . 34

2.3 .2 Spectroscopy . . . . . . . . . . . . . . . . 36

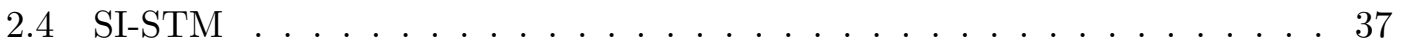


2.4.1 The Setup Effect . . . . . . . . . . . . . . . 38

2.4.2 Spectroscopic Functions $g(\vec{r}, E), I(\vec{r}, E), Z(\vec{r}, E)$, and $R(\vec{r}, E)$. . . 40

2.5 Experimental Apparatus . . . . . . . . . . . . . . . . . 42

2.5.1 STM Head . . . . . . . . . . . . . . . . . . . 42

2.5.2 Cryogenics ..................... . . 42

2.5.3 Vibration and RF Isolation . . . . . . . . . . . . . . 43

2.5.4 Instrumentation .................... 43

2.6 Cuprate Materials . . . . . . . . . . . . . . . . 44

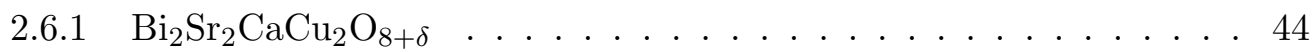

2.6.2 $\mathrm{Na}_{x} \mathrm{Ca}_{2-x} \mathrm{CuO}_{2} \mathrm{Cl}_{2}$.................... . . 46

2.7 Quasi-Particle Interference in Cuprates . . . . . . . . . . . . . 47

2.7.1 QPI - A Qualitative Understanding............. . 48

2.7 .2 The Octet Model . . . . . . . . . . . . . . . . 50

2.8 Mapping Spatial Variations in the Amplitude and Phase of LDOS Modulations ..................... 52

3 Sub-Lattice Segregated SI-STM in Cuprates 55

3.1 Data Acquisition Parameters . . . . . . . . . . . . 56

3.2 Atomic Phase Correction Algorithm . . . . . . . . . . . . . . . 57

3.3 Finding Atomic Positions . . . . . . . . . . . . . . 59

3.4 Sub-Lattice Segregation . . . . . . . . . . . . . . 60

3.5 Intra-Unit-Cell Spatial Registration of Measurements . . . . . . . . . . 62

4 Atomic-scale Electronic Structure of the Cuprate $d$-symmetry Form Factor Charge Density Wave $\quad 65$

4.1 Form Factor Decomposition of Modulations in the $\mathrm{CuO}_{2}$ Plane . . . . . . 66

4.2 Proposals for a $d$-Symmetry Form Factor CDW in Cuprates . . . . . . . . 70

4.3 SI-STM Signatures of a $d$-Symmetry Form Factor CDW . . . . . . . . . . 72

4.4 Detection of a $d$-Symmetry Form Factor CDW in Cuprates . . . . . . . . 75

4.4.1 Form Factor Measurement using Sub-Lattice Segregated SI-STM . 75

4.4.2 Temperature Dependence of the CDW Phenomena . . . . . . . . 83

4.4.3 Short-Range Unidirectional CDW Domains . . . . . . . . . . . 84

4.5 Characteristics of CDW Modulations in the Spectral Function . . . . . . . 88

4.6 Phase Relationship between the Modulation of Filled and Empty States . 91

4.7 Doping-Dependence of the CDW Wave-vector . . . . . . . . . . . . . 94

4.8 Conclusions and Proposed Future Experiments . . . . . . . . . . . . 96

5 The Scanned Josephson Tunnelling Microscope 99

5.1 Fundamentals of SJTM Operation . . . . . . . . . . . . 100

5.2 The Josephson Effect as a Measure of the Superconducting Order Parameter 103

5.3 Josephson Current-Voltage Characteristics in Ultra-Small Junctions . . 109

5.3.1 $I\left(V_{J J}\right)$ Characteristics for Josephson Junctions with Strong Phase

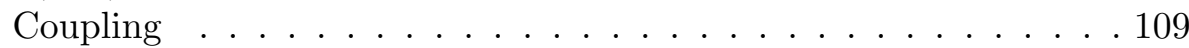

5.3.2 Thermal Phase Fluctuations in Ultra-Small Josephson Junctions . 114

5.3.3 Including both Thermal and Quantum Phase Fluctuations . . . . . 117

5.3.4 Expected Pair-Current $I_{P}(V)$ in Ultra-Small Josephson Junctions 122

5.4 Experimental Strategy for Cuprate SJTM . . . . . . . . . . . . . . . . . . 124

5.5 Superconducting STM Tips for Cuprate SJTM . . . . . . . . . . . . 124 
5.5.1 Fabrication of Superconducting $\mathrm{Bi}_{2} \mathrm{Sr}_{2} \mathrm{CaCu}_{2} \mathrm{O}_{8+\delta}$ Nano-flake Tips 125

5.5.2 Characterisation of Nano-flake Tip Geometry . . . . . . . . . . . . 126

5.5.3 Stability of Tips during $I_{c}(\vec{r})$ Measurement . . . . . . . . . . . . 129

5.6 Experimental Josephson Current-Voltage Characteristics . . . . . . . . . 130

5.7 Validating SJTM as a Probe of the Order Parameter . . . . . . . . . . . . 135

5.7.1 Imaging the Suppression of $\Psi(\vec{r})$ at Individual Zinc Impurities . . 135

5.7.2 Modulation of Superconductivity by the Bulk Structural Super-

Modulation . . . . . . . . . . . . . . 136

5.8 Conclusions and Future Directions of SJTM . . . . . . . . . . . . . 139

5.8.1 Future State of the Art in Condensate Imaging . . . . . . . . . . 139

5.8.2 Inelastic Cooper-pair Tunnelling Spectroscopy . . . . . . . . . . . . 141

6 Pair Density Waves in Cuprates 143

6.1 The Pair Density Wave . . . . . . . . . . . . . . . . . . . . . . . . . 143

6.2 Experimental Evidence for a Cuprate PDW . . . . . . . . . . . . . . . 149

6.2.1 Decoupling of Superconductivity between the $\mathrm{CuO}_{2}$ Planes . . . . 149

6.2 .2 Photoemission Spectra . . . . . . . . . . . . . . . 153

6.3 Coupled Order Parameters . . . . . . . . . . . . . . . . . . . . . 154

6.4 Coupling of PDW to Other Order Parameters . . . . . . . . . . . . . . 156

6.4 .1 Coupling to CDW . . . . . . . . . . . . . 156

6.4 .2 Coupling to SDW . . . . . . . . . . . . . . . 157

6.4.3 Coupling to Uniform Superconductivity . . . . . . . . . . . . . . 157

6.5 Theoretical Proposals for a Cuprate PDW . . . . . . . . . . . . . . . . 158

6.5.1 Variational Numerical Calculations . . . . . . . . . . . . . . . 159

6.5.2 PDW within Mean-Field Models . . . . . . . . . . . . . . . . 160

6.5.3 Amperean Pairing . . . . . . . . . . . . . . . . . . . . . . . . . . . . . . . . . . . . . . . .

6.5.4 Spin-Fermion "Hot-Spot" Models . . . . . . . . . . . . . . . . 163

6.6 Experimentally Distinguishing PDW Scenaria . . . . . . . . . . . . . . . 164

7 Detection of a Cooper-pair Density Wave in $\mathrm{Bi}_{2} \mathrm{Sr}_{2} \mathrm{CaCu}_{2} \mathrm{O}_{8+\delta} \quad 167$

7.1 Four Unit-Cell Periodic Modulations in the Cooper-Pair Current . . . . . 168

7.2 Discussion of $I_{c}(\vec{r})$ Modulations as a Pair Density Wave . . . . . . . . 171

7.3 Conclusions . . . . . . . . . . . . . . . . . . . . 173

8 Summary and Future Directions $\quad 175$

$\begin{array}{lr}\text { A Publications Arising from Doctoral Studies } & 179\end{array}$

A.1 Piezoelectric Strain Tuning . . . . . . . . . . . . . . . . . . . . . 179

A.2 Spectroscopic Imaging STM of the Cuprate CDW . . . . . . . . . . . 179

A.3 Scanned Josephson Tunnelling Microscopy . . . . . . . . . . . . . . . 180

B A Form Factor Description of Local Rotational Symmetry in Density Waves

$\begin{array}{ll}\text { C Calculating Sub-lattice Fourier Transforms } & 185\end{array}$

$\begin{array}{ll}\text { D Sub-Lattice Phase-Resolved Fourier Transforms } & 191\end{array}$ 
E Topographic Imaging of the Cuprate $d$-Symmetry Form Factor Charge Density Wave 193

F Absence of Setup Effect in Relative Spatial Phase Measurements 197

G Antiphase Modulation of Filled and Empty States at $|E|=\Delta_{1}$ 201

H Measuring $R_{N}$ for Cuprate SJTM 203

Bibliography 207 


\section{List of Figures}

1.1 Phase Stiffness in a $1 \mathrm{D}$ Crystal $\ldots \ldots \ldots \ldots . \ldots \ldots$

1.2 Bogoliubov Quasi-Particles . . . . . . . . . . . . . . . . . . . 9

1.3 The $\mathrm{CuO}_{2}$ Plane ......................... . . 10

1.4 A Charge Transfer Insulator . . . . . . . . . . . . . . . . . . . 11

1.5 Cuprate Phase Diagram . . . . . . . . . . . . . . . . . . . 14

$1.6 d$-Wave Superconducting Gap and Pseudogap . . . . . . . . . . . . . . 15

1.7 Bare Particle-Hole Susceptibility in $\mathrm{d}=1,2$ \& $3 \ldots \ldots$. . . . . . . . . 18

1.8 CDW Enhancement upon Suppressing Superconductivity . . . . . . . . . 22

2.1 Schematic of Vacuum Tunnelling . . . . . . . . . . . . . . . . . . . . . . 29

2.2 Schematic of STM Operation . . . . . . . . . . . . . . 33

2.3 Atomically Resolved Topograph . . . . . . . . . . . . . . . . 35

2.4 STM Tunnelling Conductance Spectrum . . . . . . . . . . . . . 36

2.5 IIllustration of SI-STM . . . . . . . . . . . . . . . . . . . . . 38

2.6 The Setup Effect . . . . . . . . . . . . . . . . . . . . 40

2.7 STM Head . . . . . . . . . . . . . . . . . . . . . . . . . . . . . . . . . . . . . . .

2.8 Passive Vibration Isolation . . . . . . . . . . . . . . . . . 44

2.9 SI-STM Instrumentation . . . . . . . . . . . . . . . . . . . . . . . 45

2.10 Unit Cell of $\mathrm{Bi}_{2} \mathrm{Sr}_{2} \mathrm{CaCu}_{2} \mathrm{O}_{8+\delta} \ldots \ldots \ldots \ldots \ldots$. . . . . . . . . . 47

2.11 Unit Cell of $\mathrm{Na}_{x} \mathrm{Ca}_{2-x} \mathrm{CuO}_{2} \mathrm{Cl}_{2} \ldots \ldots \ldots \ldots$. . . . . . . . . 48

2.12 The Octet Model . . . . . . . . . . . . . . . . . . . . . 50

$2.13 d$-Wave Superconducting Gap and Fermi Surface in $\mathrm{Bi}_{2} \mathrm{Sr}_{2} \mathrm{CaCu}_{2} \mathrm{O}_{8+\delta} \ldots 53$

3.1 Sub-lattice Segregation for $\mathrm{Na}_{x} \mathrm{Ca}_{2-x} \mathrm{CuO}_{2} \mathrm{Cl}_{2} \quad \ldots \ldots \ldots$. . . . . . . 61

3.2 Spatially Registered $\mathrm{B}=8.5 \mathrm{~T}$ and $\mathrm{B}=0 \mathrm{~T}$ Topographs . . . . . . . . . . 63

4.1 The Three Sub-lattices in the $\mathrm{CuO}_{2}$ Plane . . . . . . . . . . . . . . 66

4.2 Intra-Unit-Cell Electronic Structure Symmetry in the $\mathrm{CuO}_{2}$ Plane . . . . 68

4.3 Types of $\mathrm{CuO}_{2}$ Intra-Unit-Cell Density Waves . . . . . . . . . . . . . . 71

4.4 Experimental Signatures of $d$-Symmetry Form Factor CDW . . . . . . . . 74

4.5 Oxygen site-specific imaging and segregation of $R(\vec{r}) \ldots \ldots$. . . . . . 77

4.6 Sub-lattice Segregation for $\mathrm{Bi}_{2} \mathrm{Sr}_{2} \mathrm{CaCu}_{2} \mathrm{O}_{8+\delta} \ldots \ldots \ldots \ldots . \ldots . \ldots 78$

4.7 Sub-lattice Phase-Resolved Fourier analysis revealing a $d$-Symmetry Form Factor . . . . . . . . . . . . . . . . . . . . 79

4.8 Predominance of the $d$-Symmetry Form Factor . . . . . . . . . . . . 81

4.9 Representative Unidirectional Domains CDW . . . . . . . . . . . . . . 82

4.10 Unidirectional $d$-Symmetry Form Factor CDW Domains in the Superconducting and pseudogap phases. . . . . . . . . . . . . 85 
4.11 Underdoped Cuprate Tunnelling Spectrum . . . . . . . . . . . . . . . 88

4.12 Dispersion of Bogoliubov Quasi-particle and CDW Modulations . . . . . . 89

4.13 PSD for Measuring Form Factor Amplitudes . . . . . . . . . . . . . . . . . 90

4.14 Equivalent representation of $d$-symmetry Form Factor modulations ‥ 91

4.15 Energy Dependence of Form Factor Amplitudes . . . . . . . . . . . . . 92

4.16 Relationship between CDW Modulations of filled and empty states . . . . 93

4.17 Doping Dependence of Fermi Surface and CDW Wave-Vector . . . . . . . 95

5.1 Conceptual Operation of SJTM . . . . . . . . . . . . . . . . . . . 101

5.2 The RCSJ Model . . . . . . . . . . . . . . . . . . . . . . 110

5.3 The Tilted Washboard Potential _. . . . . . . . . . . . . . . . 111

$5.4 I\left(V_{J J}\right)$ for Coherent Phase Dynamics . . . . . . . . . . . . . . . 112

5.5 Lumped Element Representation of Shunting by Electromagnetic Environment . . . . . . . . . . . . . . . . . . . . . . . . 114

5.6 The Effect of a Parasitic Capacitance . . . . . . . . . . . . . . 116

$5.7 \quad I\left(V_{J J}\right)$ with Strong Thermal Fluctuations . . . . . . . . . . . . 117

5.8 Inelastic Cooper-pair Tunnelling. . . . . . . . . . . . . . . . . . . 120

$5.9 I\left(V_{J J}\right)$ with Strong Quantum and Thermal Phase Fluctuations . . . . . . 123

5.10 Single-Particle Tunnelling Spectrum with Superconducting Tip . . . . . . 125

5.11 Schematic of SIS Single-Particle Tunnelling Between $d$-Wave Superconductors . . . . . . . . . . . . . . . . . . 127

5.12 Spectroscopic/topographic data from two distinct $\mathrm{Bi}_{2} \mathrm{Sr}_{2} \mathrm{CaCu}_{2} \mathrm{O}_{8+\delta}$ tips . 128

5.13 Simulation of topography with BSCCO nanoflake tip . . . . . . . . . . 129

5.14 Demonstration of Tip Stability during SJTM Operation . . . . . . . . . . 130

5.15 SJTM circuit model . . . . . . . . . . . . . . . . . . . . . . . . . . . . . . . . . . . 132

5.16 Experimental SJTM $I\left(V_{J J}\right)$ Characteristics . . . . . . . . . . . . 134

5.17 Typical $I_{c}(\vec{r})$ Measurement . . . . . . . . . . . . . 135

5.18 The Suppression of $I_{c}$ at Individual Zinc Impurities . . . . . . . . . . . 137

5.19 Sequence of measured Ic(r) along line through a Zinc Impurity . . . . . . 138

5.20 Spatial Variations and Modulations in Cuprate Energy Gaps . . . . . . . 139

$5.21 I_{c}(\vec{r})$ Modulation due to Crystal Super-modulation . . . . . . . . . . . . 140

6.1 Cooper Pairing at Non-Zero Centre of Mass Momentum . . . . . . . . . . 145

6.2 Formation of the FFLO State from Zeeman Split Fermi Surfaces . . . . . 147

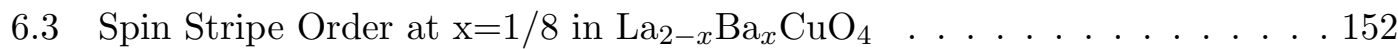

6.4 PDW like Features of the Anti-Nodal Gap in $\mathrm{Bi}_{2} \mathrm{Sr}_{2} \mathrm{CuO}_{6+\delta} \ldots . . . .154$

6.5 Striped Variational Solutions to the $t$ - $J$ Model . . . . . . . . . . . . . . . 159

6.6 Amperean Pairing . . . . . . . . . . . . . . . . . . . . . 162

6.7 Cartoon of Orthogonal CDW/PDW State in a Spin-Fermion Model . . . 163

7.1 Nanometer Resolution Imaging of the Superconducting Condensate by SJTM . . . . . . . . . . . . . . . . . . . . 169

7.2 Visualising the Cooper Pair Density Wave in $\mathrm{Bi}_{2} \mathrm{Sr}_{2} \mathrm{CaCu}_{2} \mathrm{O}_{8+x} \ldots \ldots . .170$

C.1 Sub-lattice decomposition of $d$-Symmetry Form Factor CDW . . . . . . . 187

C.2 Fourier Analysis of CDW using the Convolution Theorem . . . . . . . . . 189

D.1 Sub-Lattice Segregated Fourier Analysis of CDW in $\mathrm{Bi}_{2} \mathrm{Sr}_{2} \mathrm{CaCu}_{2} \mathrm{O}_{8+\delta} \ldots 191$ 
D.2 Sub-Lattice Segregated Fourier Analysis of CDW in $\mathrm{Na}_{x} \mathrm{Ca}_{2-x} \mathrm{CuO}_{2} \mathrm{Cl}_{2} \quad .192$

E.1 d-Symmetry Form Factor CDW Visualised through Topography . . . . 195

F.1 Establishing the Absence of a Setup Effect using Simultaneous Topogra-

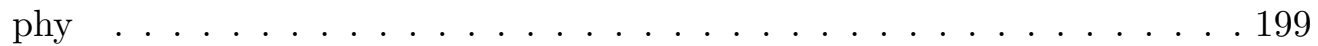

G.1 Energy Dependent Phase Difference . . . . . . . . . . . . . . . . . 202

H.1 Comparison of modulations in $I_{c}(\vec{r})$ and $R_{N}(\vec{r}) \ldots \ldots \ldots$ 



\section{List of Tables}

6.1 PDW Phases in a Quasi Two-Dimensional Tetragonal Lattice . . . . . . . 149

6.2 PDW Scenaria in Cuprates . . . . . . . . . . . . . . . . . 165 

In memory of my father, David John Edkins (1947-2007), who fostered my interest in science and technology. 



\section{Chapter 1}

\section{Unconventional}

\section{Superconductivity and Density}

\section{Wave Order in Cuprates}

Superconductors, known for their ability to conduct electricity without resistance, are fairly common in nature. This is a result of the fact that, despite the strong interactions between their electrons, most of the metals we know can be described by a liquid of electron-like quasi-particles. This liquid, known as a Fermi liquid, is intrinsically unstable to the formation of superconductivity. The vast majority of these superconductors only superconduct below a transition temperature which is within a few degrees of absolute zero, limiting their widespread commercial exploitation.

This thesis concerns a family of superconductors whose superconducting transition temperatures were unprecedentedly high upon their discovery by Bednorz and Muller in 1986 [1]. These materials, known as cuprates because their common constituents are copper and oxygen, won Bednorz and Muller the 1987 Nobel Prize for Physics for their discovery [2]. Although the cuprates lost their status as the highest temperature superconductors in the past couple of years to hydrides at ultra-high pressures, they retain the name "high temperature superconductors" and are still a system of unparalleled interest [3]. The high transition temperatures of the hydrides seems to be well understood in terms of extensions of traditional theory, but the mechanism of the more exotic superconductivity in cuprates is still the subject of active debate. 
In addition to superconductivity, the cuprates also exhibit what is called a charge density wave $(\mathrm{CDW})$. In a CDW the charge density in the material modulates with a periodicity that is different from that of the material's crystal structure. In this thesis I utilise recent developments in scanning tunnelling microscopy (STM), and develop nanometerresolution scanned Josephson tunnelling microscopy (SJTM) with the aim of elucidating the role of CDW in the physics of these materials.

\section{$1.1 \quad$ Superconductivity}

\subsubsection{What is a Superconductor?}

The concept of spontaneous symmetry breaking is a cornerstone of 20th century physics [4]. In condensed matter physics we have chosen to classify different phases of matter by the symmetries they break. If the state of a system is not invariant under an element of the symmetry group of the Hamiltonian to which it is a statistical solution, then it is said to break the corresponding symmetry.

As an example take a macroscopic number of particles in free space which interact with each other. The Hamiltonian which governs these particles is invariant under spatial translations. However, the particles could condense into a crystal. This is clearly a state which breaks translational symmetry as depicted in figure 1.1 in one dimension.

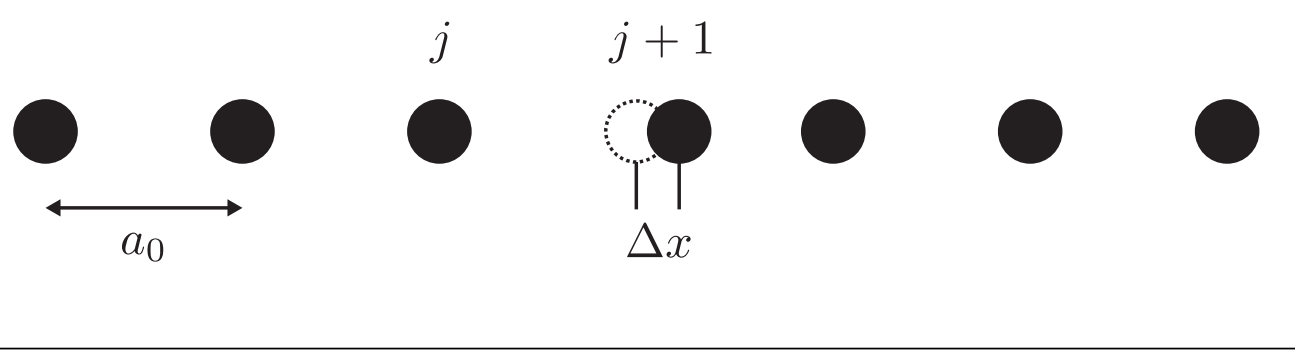

Figure 1.1: Cartoon one-dimensional crystal with lattice constant $a_{0}$. The distance between the $j$ th and $j+1$ th particle is stretched by a distance $\Delta x$.

The crystal has broken translational symmetry by "choosing" where to fix the $j$ th particle in space, the positions of the others following directly from the lattice constant $a_{0}$ in a perfect crystal. Any choice of position for this first particle would have resulted in a state with the same free energy. The crystal has spontaneously broken translational symmetry by its choice of a position for the $j$ th particle from a degenerate manifold. 
We can expand the density of particles in Fourier components,

$$
\langle n(x)\rangle=n_{0}+\operatorname{Re}\left\{n_{Q}(x) e^{i Q x}\right\}+\ldots,
$$

where $Q=2 \pi / a_{0}$. The complex field $n_{Q}(x)=\left|n_{Q}(x)\right| e^{i \arg \left\{n_{Q}(x)\right\}}$ is a sensible choice of order parameter for our crystalline phase. It will be zero above the melting temperature of the crystal and non-zero below. In this language, choosing a particular position for the $j$ th particle corresponds to choosing a particular phase of $n_{Q}(x), \phi(x)=\arg \left\{n_{Q}(x)\right\}$. This corresponds to spontaneously breaking a U(1) symmetry. The concept of spatial phase in a crystal will be a key theme in this thesis which I will return to in chapters 3 and 4, where the spatial phase sensitivity of spectroscopic-imaging STM (SI-STM) will be used to probe the intra unit cell electronic structure of cuprates.

Part of the utility of the classifying phases of matter by their broken symmetries is that the broken symmetry has an attendant generalised rigidity [4], i.e a stiffness to spatial gradients in the order parameter. For the crystal there is a very tangible rigidity to changing the separation between any two atoms, akin to stretching a spring. If we consider the order parameter description of this crystal then we can recast this as a phase rigidity. To change the distance between two particles, $j$ and $j+1$ by an amount $\Delta x$ requires a phase gradient $\phi\left(x_{j+1}\right)-\phi\left(x_{j}\right)=Q \Delta x$, for which there is an energy cost.

If we make the constraint that the order parameter amplitude is spatially uniform so that $n_{Q}(x)=\left|n_{0}\right| e^{i \phi(x)}$ we can make the following expansion of the free energy

$$
F[\phi(x)]=F_{0}+\int d x K(\nabla \phi(x))^{2}+\ldots
$$

where $K$ is a positive constant and $F_{0}$ is the energy of the uniform crystal. Here there is a clear energy cost to gradients in $\phi(x)$; a phase rigidity. Viewed this way, that fact that crystals behave like rigid bodies is a natural consequence of their phase stiffness, which in turn is a direct consequence of breaking translational symmetry.

For a superconductor the relevant broken symmetry of the Hamiltonian is a global phase symmetry. This is the invariance of the Hamiltonian under a transformation of the quasiparticle operators of the form $\hat{\psi}^{\dagger}(\vec{r}) \rightarrow e^{i \theta} \hat{\psi}^{\dagger}(\vec{r})$, as must be the case if the number of particles is conserved. What are the consequences of breaking this symmetry? 
An order parameter describing this phase would be a complex scalar field of the form $\Psi(\vec{r})=|\Psi(\vec{r})| e^{i \phi(\vec{r})}$. If this order parameter is non-zero then everywhere this state breaks the $\mathrm{U}(1)$ phase symmetry of the Hamiltonian. We can construct a Ginzburg-Landau expansion of the Helmholtz free energy functional in this order parameter

$$
F[\Psi(\vec{r})]=\int d^{3} \vec{r} \alpha\left(T-T_{c}\right)|\Psi(\vec{r})|^{2}+\frac{\beta}{2}|\Psi(\vec{r})|^{4}+\frac{1}{2 m}|(-i \hbar \nabla-2 e \vec{A}) \Psi(\vec{r})|^{2}+\frac{|\vec{B}|^{2}}{2 \mu_{0}}+\ldots
$$

where $\vec{A}$ is the magnetic vector potential and $\vec{B}=\nabla \times \vec{A}[5]$. This is the simplest expansion of an electrically charged complex scalar field that is gauge invariant. In anticipation of the microscopic description of a superconductor in terms of Cooper-pairs of electrons I have given the field a charge $2 e$ where $e$ is the electron charge. That the field is scalar implies that these Cooper-pairs are in a spin-singlet state. Below a critical temperature $T_{c}$ the order parameter $\Psi$ will become non-zero to minimise this free energy.

Now consider the case where the order parameter amplitude is everywhere constant so that $\Psi(\vec{r})=\left|\Psi_{0}\right| e^{i \theta(\vec{r})}$. We can write the free energy functional as,

$$
F[\theta(\vec{r})]=F_{0}+\rho_{s} \int d^{3} \vec{r}\left(\nabla \theta(\vec{r})+\frac{2 e}{\hbar} \vec{A}\right)^{2}+\ldots
$$

with $F_{0}$ the free energy of the uniform superconducting state and $\rho_{s}$, the superfluid stiffness, given by

$$
\rho_{s}=\frac{\hbar^{2}}{2 m}\left|\Psi_{0}\right|^{2}
$$

It is informative at this point to revisit the discussion of crystallisation and the analogies that can be drawn between that process and the formation of the superconducting condensate. The $1 \mathrm{D}$ crystal considered above breaks a global U(1) symmetry by choosing a lattice phase. This broken symmetry results in a generalised rigidity, a rigidity against deforming the inter-particle separation from its equilibrium value. This can be expressed as a phase stiffness. This phase stiffness essentially defines this 1D crystalline state of matter.

The same is true of the superconductor. It breaks a $\mathrm{U}(1)$ phase symmetry resulting in a stiffness, $\rho_{s}$, to gradients in the phase of the order parameter $\theta(\vec{r})$. Below I will show that the physical properties of a superconductor follow immediately from this broken 
phase symmetry and its resultant phase stiffness. This phase stiffness is the defining property of a superconductor.

Making a Legendre transformation, $g=f-\mu_{0} \vec{H} \cdot \vec{B}$, of equation 1.4 to the Gibbs free energy and minimising with respect to variations $\delta \vec{A}$ yields a current

$$
\vec{j}_{s}=-\frac{2 e}{\hbar} \rho_{s}\left(\nabla \theta(\vec{r})+\frac{2 e}{\hbar} \vec{A}\right)
$$

This expression has profound consequences.

The first is that superconductors should expel magnetic flux from their bulk: the Meissner effect [6]. To see this we can take the curl of both sides resulting in the following differential equation for the magnetic field

$$
\nabla^{2} \vec{B}=\frac{\vec{B}}{\lambda^{2}}
$$

where $\lambda=\sqrt{\hbar^{2} / 4 \mu_{0} e^{2} \rho_{s}}$ is a phenomenological constant called the penetration depth. This implies that magnetic fields must exponentially decay into the bulk of a superconductor over a length scale $\lambda$. Thus superconductors expel magnetic fields from their bulk.

To act as perfect diamagnets and expel magnetic fields from their bulk, superconductors must produce screening currents that oppose an applied magnetic field. These must be equilibrium currents, implying that they experience zero resistance. These zero resistance equilibrium currents suggest the possibility of non-equilibrium currents with zero resistance which are a hallmark of superconductivity and give it its name.

A zero resistance transport current, the passing of current without any voltage dropped, was measured by Kamerlingh Onnes in mercury in 1911, heralding the discovery of superconductivity [7]. Clearly this property is remarkable and of great technological importance. However, it is not fundamental to superconductivity in the way that the equilibrium screening currents of the Meissner effect are.

\subsubsection{BCS Theory of Superconductivity}

Having considered what a superconductor is from a phenomenological point of view, I now turn to a microscopic description of such a state. Key insight into this came in 1957 
with a paper from Bardeen, Cooper and Schrieffer, the exposition of what is now called the BCS theory of superconductivity [8].

Prior to this Cooper had shown that, in the presence of a Fermi surface and arbitrarily weak attractive interactions, two quasi-particles with opposite momenta are unstable to the formation of a bound state or Cooper pair. One way of demonstrating this instability of the Fermi liquid is to calculate the particle-particle (pairing) susceptibility within the random phase approximation (RPA),

$$
\chi_{p p}(\vec{q}, \omega)=\frac{\chi_{p p}^{0}(\vec{q}, \omega)}{1+g \chi_{p p}^{0}(\vec{q}, \omega)},
$$

which measures the response to a field that acts to pair particles [9]. Here g is an effective point-like interaction between Fermi liquid quasi-particles with spin $\sigma$ of momentum $\vec{k}$ and energy $\epsilon_{\sigma, \vec{k}} \cdot \chi_{p p}^{0}$ is the bare (non-interacting) susceptibility,

$$
\chi_{p p}^{0}(\vec{q}=0, \omega)=\frac{1}{\Omega} \sum_{\vec{k}} \frac{f\left(\epsilon_{\sigma, \vec{k}}\right)-f\left(-\epsilon_{\sigma-\vec{k}}\right)}{\hbar \omega-\epsilon_{\sigma, \vec{k}}-\epsilon_{-\sigma,-\vec{k}}+i 0^{+}},
$$

where $f\left(\epsilon_{\sigma, \vec{k}}\right)$ is the occupation factor of a quasi-particle state labelled by $\vec{k}$ and $\sigma$ and $\Omega$ is the volume of the system.

In the limit where $\omega, \vec{q} \rightarrow 0$

$$
\chi_{p p}^{0}(\vec{q}=0, \omega=0) \sim N(\epsilon=0) \log (\Lambda / T) .
$$

Here $N(\epsilon=0)$ is the density of states of the Fermi energy and $\Lambda$ is an ultra-violet cut-off [9]. Equation 1.10 shows that $\chi_{p p}^{0}(\vec{q}=0, \omega=0)$ is positive and logarithmically divergent with decreasing temperature. This results from the fact that, in the presence of time reversal symmetry, $\epsilon_{\sigma, \vec{k}}=\epsilon_{-\sigma,-\vec{k}}$. Examination of equation 1.8 then shows that for any arbitrarily small attractive interaction $(g<0)$ the particle-particle susceptibility will diverge at non-zero temperature. This signals an intrinsic instability of the metal towards forming Cooper pairs.

In the simplest case, the wave-function of the Cooper-pair can be written as a product of orbital and spin parts. Anti-symmetry under exchange then dictates that if the pair is in a spin-singlet state it must have an even parity orbital wave-function. If the pair is a spin-triplet state it must have an odd parity orbital wave-function. If we were to 
expand the orbital wave-function in spherical harmonics (which is valid in free space), spin-singlet pairs will have angular momentum quantum number $l=0,2,$. which we call $s$ - and $d$-wave respectively, by analogy with the orbitals of the hydrogen atom. Likewise, spin-triplet superconductors will have $l=1,3, \ldots$ corresponding to $p$ - and $f$-wave respectively.

With the insight that the Fermi liquid is unstable to the formation of Cooper-pairs in the presence of an attractive interaction, Bardeen, Cooper and Schrieffer considered the equivalent of the Hamiltonian,

$$
\hat{H}=\sum_{\vec{k} \sigma} \epsilon_{\vec{k}} \hat{c}_{\vec{k} \sigma}^{\dagger} \hat{c}_{\vec{k} \sigma}+\sum_{\vec{k} \vec{q}} V_{\vec{k} \vec{q}} \hat{c}_{\vec{k} \uparrow}^{\dagger} \hat{c}_{-\vec{k} \downarrow}^{\dagger} \hat{c}_{-\vec{q} \downarrow} \hat{c}_{-\vec{q} \uparrow}
$$

where

$$
V_{\vec{k} \vec{q}}= \begin{cases}-V & \text { if }\left|\epsilon_{\vec{k}}\right| \text { and }\left|\epsilon_{\vec{q}}\right| \leq \hbar \omega_{c} \\ 0 & \text { otherwise }\end{cases}
$$

so that there is an attractive interaction between quasi-particles up to a energy cut-off $\hbar \omega_{c}$ away from the Fermi energy. It is manifest in the form of the interaction chosen that any Cooper pairs formed will be of the spin-singlet type and thus must have an even parity orbital wave-function.

Anticipating the formation of a condensate of Cooper-pairs BCS introduced the variational wave-function,

$$
\left|\Psi_{B C S}\right\rangle=\prod_{\vec{k}}\left(\left|u_{\vec{k}}\right|+\left|v_{\vec{k}}\right| e^{i \theta} \hat{c}_{\vec{k} \uparrow}^{\dagger} \hat{c}_{-\vec{k} \downarrow}^{\dagger}\right)|0\rangle,
$$

where $\left|u_{\vec{k}}\right|^{2}+\left|v_{\vec{k}}\right|^{2}=1, \theta$ is an arbitrary phase factor and $|0\rangle$ corresponds a sea of Fermi liquid quasi-particle states filled up to the Fermi wave-vector $\vec{k}_{F}$ [8]. This many-body wave-function takes the form of a superposition of a filled Fermi surface plus a Fermi surface with $0,1,2, \ldots$ Cooper pairs. Making a connection with the previous section, this is a coherent state of Cooper pairs, with minimum uncertainty in the phase and indefinite particle number. It thus manifestly breaks phase symmetry and we can associate $\theta$ with the phase of the condensate.

BCS went on to find the parameters $u_{\vec{k}}$ and $v_{\vec{k}}$ of the ground state by the variational method. In chapter 2 we will need to calculate the single-particle tunnelling current 
between a superconductor and either a normal metal or another superconductor. For this we will need to know the single-particle excitation spectrum of the superconductor. To calculate this one can follow Bogoliubov [10] who considered the following mean-field decoupling of equation 1.11

$$
\hat{H}=\sum_{\vec{k} \sigma} \epsilon_{\vec{k}} \hat{c}_{\vec{k} \sigma}^{\dagger} \hat{c}_{\vec{k} \sigma}+\sum_{\vec{k} \sigma} \Delta_{\vec{k}} \hat{c}_{\vec{k} \uparrow}^{\dagger} \hat{c}_{-\vec{k} \downarrow}^{\dagger}+\Delta_{\vec{k}}^{*} \hat{c}_{-\vec{k} \downarrow} \hat{c}_{-\vec{k} \uparrow}
$$

with mean-field $\Delta_{\vec{k}}$ given by the self-consistency condition

$$
\Delta_{\vec{k}}=\sum_{\vec{q}} V_{\vec{k} \vec{q}}\left\langle\hat{c}_{-\vec{q} \downarrow} \hat{c}_{\vec{q} \uparrow}\right\rangle
$$

The Hamiltonian in equation 1.14 can be diagonalised by a canonical transformation. First we introduce new quasi-particle operators which are mixtures of electron and hole creation operators,

$$
\begin{aligned}
& \alpha_{\vec{k}}=u_{\vec{k}} \hat{c}_{\vec{k}, \uparrow}-v_{\vec{k}} \hat{c}_{-\vec{k}, \downarrow}^{\dagger} \\
& \beta_{\vec{k}}=u_{\vec{k}} \hat{c}_{-\vec{k}, \downarrow}+v_{\vec{k}} \hat{c}_{\vec{k}, \uparrow}^{\dagger},
\end{aligned}
$$

where $\left|u_{\vec{k}}\right|^{2}+\left|v_{\vec{k}}\right|^{2}=1$ to retain Fermionic anti-commutation relations. In terms of these operators the Hamiltonian can be written in the diagonal form,

$$
\hat{H}=\sum_{k} E_{k}\left(\alpha_{\vec{k}}^{\dagger} \alpha_{\vec{k}}+\beta_{\vec{k}}^{\dagger} \beta_{\vec{k}}\right)
$$

where $E_{\vec{k}}=\sqrt{\epsilon_{\vec{k}}^{2}+\left|\Delta_{\vec{k}}\right|^{2}}$ and the coefficients $u_{\vec{k}}$ and $v_{\vec{k}}$ that diagonalise the Hamiltonian are

$$
\left|v_{\vec{k}}\right|^{2}=1-\left|u_{\vec{k}}\right|^{2}=\frac{1}{2}\left(1-\frac{\epsilon_{\vec{k}}}{E_{\vec{k}}}\right) .
$$

Equation 1.18 shows that there is a gap $\Delta_{k}$ to excitations of the superconductor as shown in figure 1.2. On exceeding the gap energy, the excitations are described by acting on the BCS ground state with a mixture of particle and hole creation operators. For example $\alpha_{\vec{k}}^{\dagger}$ creates a quasi-particle which for $E_{\vec{k}}=\Delta_{\vec{k}}$ is an equal admixture of electron and hole creation operators, but with increasing $E_{\vec{k}}$ the admixture of hole creation operator gradually diminishes. These quasi-particles, created by $\alpha_{\vec{k}}^{\dagger}$ and $\beta_{\vec{k}}^{\dagger}$ are often called Bogoliubov quasi-particles or Bogoliubons. 


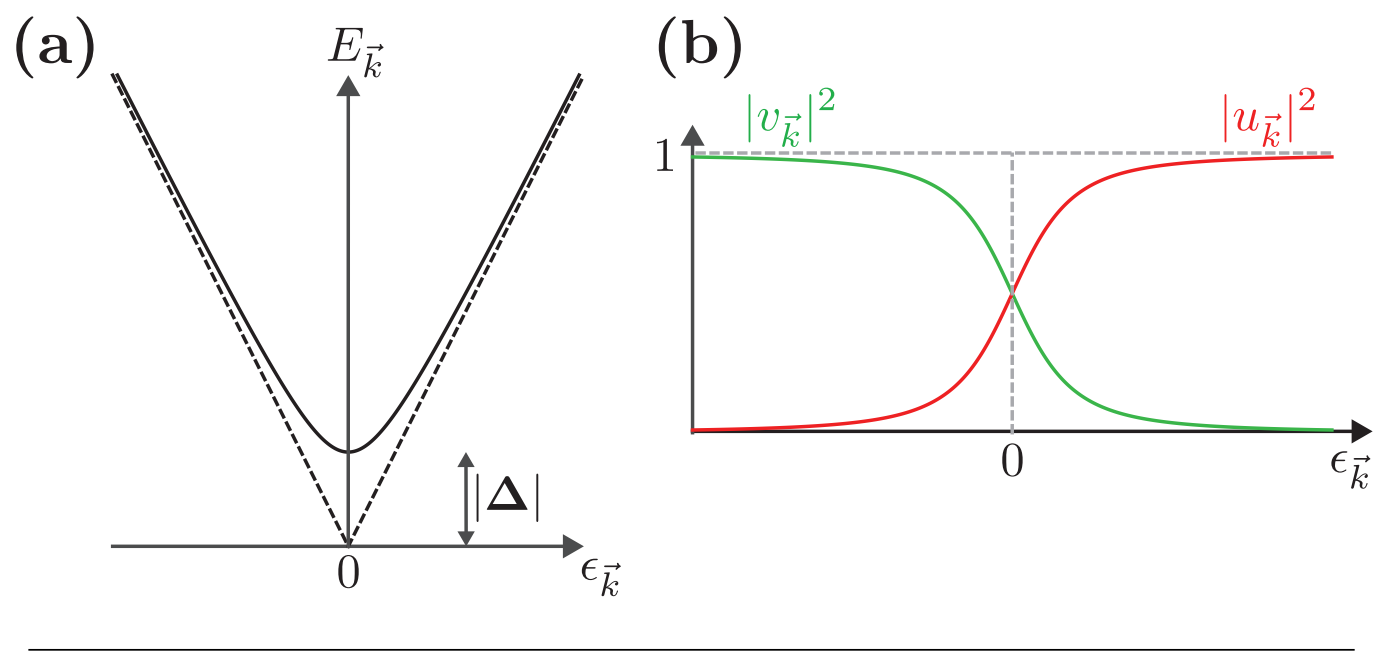

Figure 1.2: (a) Excitation spectrum for BCS superconducting state with $\epsilon_{\vec{k}}=v_{F}(\vec{k}-$ $\vec{k}_{F}$ ) and $s$-wave superconducting gap $\Delta_{k}=\Delta$. Dashed lines are the bare band $\left|\epsilon_{\vec{k}}\right|$. (b) $\left|u_{\vec{k}}\right|^{2}$ and $\left|v_{\vec{k}}\right|^{2}$ for the excitation spectrum shown in (a). At $E_{\vec{k}}=|\Delta|$ the Bogoliubov quasi-particle creation operators are an equal admixture of hole and electron creation operators but with increasing energy rapidly becomes the excitations of the bare band.

What form does this gapping of the Fermi surface take? As I will discuss below, the conventional superconductors have an $s$-wave gap that has the same sign on all parts of the Fermi surface, leading to a fully gapped Fermi surface. Cuprate superconductors on the other hand have a gap that changes sign upon rotation by $90^{\circ}$, and thus necessarily has points where the gap goes through zero called nodes [11]. At these points there are gapless Bogoliubov quasi-particle excitations.

In the conventional superconductors considered by BCS, the effective attractive interaction is a result of the electron-phonon interaction. An electron moving through the lattice disturbs it, leaving a region of net positive charge in its wake. Another electron passing through will feel an attraction to this cloud of positive charge through the Coulomb interaction. Thus, there is an effective attractive interaction between the two electrons at the same point but at different times.

This retarded interaction is afforded by the mismatch is timescales over which electrons and phonons propagate in the material. That the electrons feel an attractive interaction at the same place leads the pair-wave function to be $s$-wave so that the probability of both electrons being in the same place is non-zero, maximising the potential energy gain. Key experiments confirming the predictions of BCS theory and the electron-phonon mechanism are reviewed in reference [12]. 
Having discussed the nature of and mechanism behind conventional superconducting states I will now go to discuss the cuprate high temperature superconductors whose superconductivity in unconventional in both its nature and mechanism.

\subsection{High-Temperature Unconventional Superconductivity in Cuprates}

The common constituent of all cuprate superconductors are the copper oxide planes shown in figure 1.3 (a). These copper oxide layers are incorporated between inert ionic layers which can act as a source of holes or electrons for the $\mathrm{CuO}_{2}$ layers, thereby doping them.

(a)

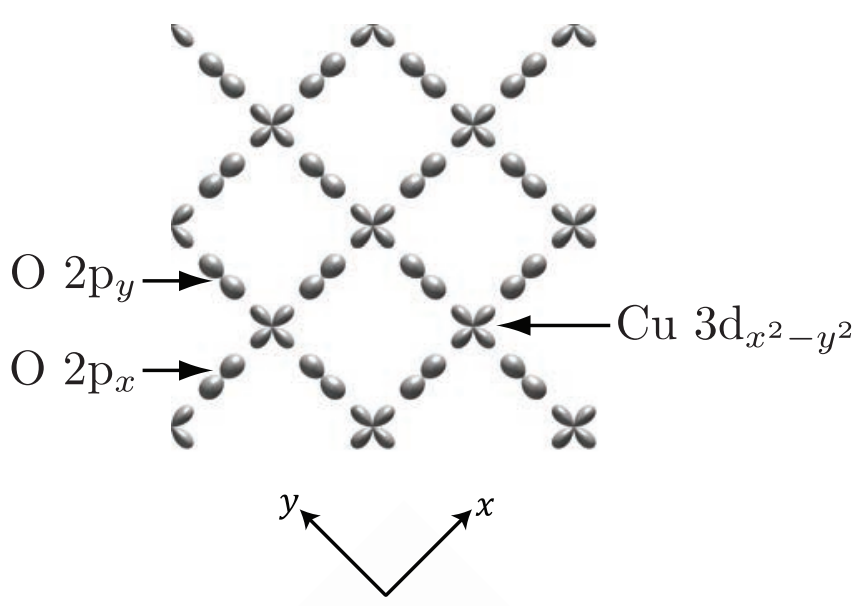

(b)

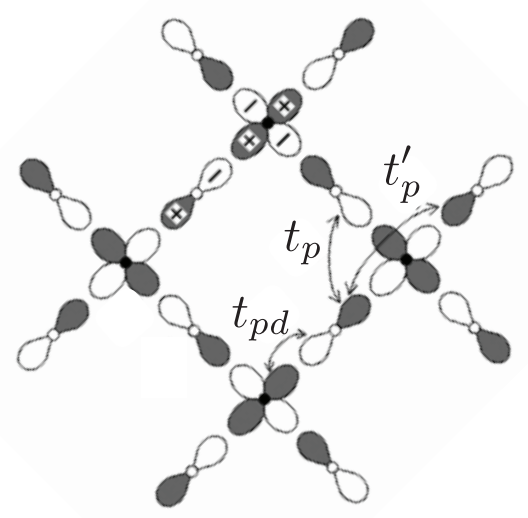

Figure 1.3: (a) $\mathrm{CuO}_{2}$ plaquettes consisting of copper $3 d_{x^{2}-y^{2}}$ and oxygen $2 \mathrm{p}_{x, y}$ orbitals forming a square lattice plane. (b) Arrows indicate orbitals contributing the the hopping integrals $t_{p d}, t_{p}$ and $t_{p}^{\prime}$. Reproduced from reference [13] with permission. 
Each $\mathrm{CuO}_{2}$ plaquette contains one copper atom and two oxygen atoms. Much focus in this thesis will be given to a breaking of rotational symmetry within the unit cell via an inequivalence of these two oxygen sites, which I will term $x$ and $y$-directed oxygens respectively.

Each copper contributes a singly occupied $3 \mathrm{~d}_{x^{2}-y^{2}}$ orbital. $x$ and $y$ directed oxygens each contribute a doubly occupied $2 \mathrm{p}_{x}$ or $2 \mathrm{p}_{y}$ orbital respectively. The compact nature of the $3 \mathrm{~d}_{x^{2}-y^{2}}$ orbital leads to a large energetic cost for double occupation arising from the Coulomb interaction. In cuprates the difference in energy between a singly and doubly occupied $3 \mathrm{~d}_{x^{2}-y^{2}}$ orbital, $\mathrm{U}_{d}$, is $\sim 7-10 \mathrm{eV}[13]$.

This onsite Coulomb repulsion, or Hubbard $U_{d}$, splits the spectral weight from the $3 \mathrm{~d}_{x^{2}-y^{2}}$ orbitals into upper and lower Hubbard bands with a gap, $U_{d}$, for charge excitations between them as shown in figure 1.4. If this were the full story then this would lead to an insulator of the Mott-Hubbard type where hopping of electrons is prohibited by the energetic cost of moving an electron from one $\mathrm{Cu}$ sites to doubly occupy another. Cuprates however fall into a related class of insulators, proposed by Zaanen, Sawatsky and Allen, known as charge-transfer insulators [14].
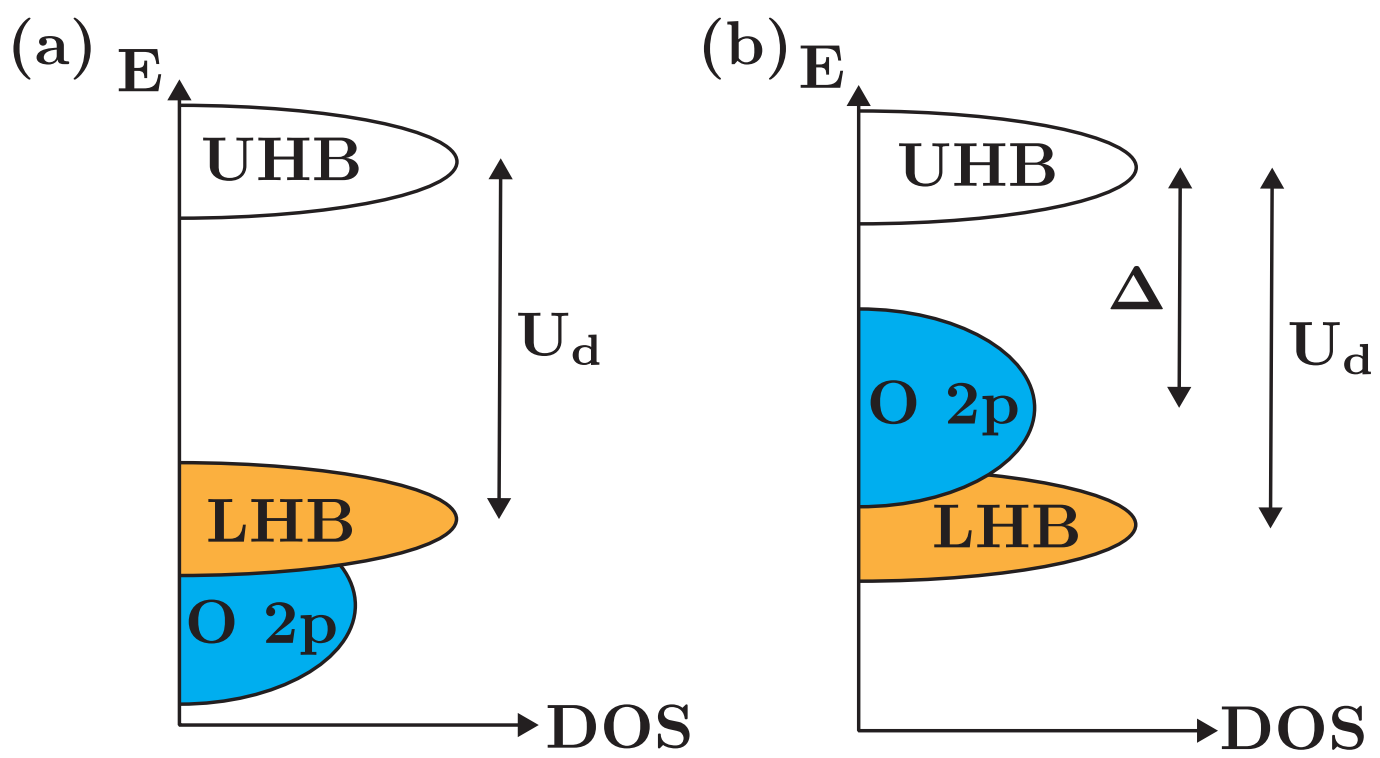

Figure 1.4: (a) Schematic depiction of single-particle spectrum for a Mott-Hubbard insulator showing smallest optical gap, $U_{d}$, is between the lower and upper Hubbard bands (UHB and LHB) derived from the $\mathrm{Cu} 3 \mathrm{~d}_{x^{2}-y^{2}}$ orbitals. (b) Depiction of a charge transfer insulator where the smallest optical gap, $\Delta$, is between the $\mathrm{O} 2 \mathrm{p}$ derived band and the upper Hubbard band (UHB). 
In a charge-transfer insulator the smallest gap for charge excitations is to take an electron from an oxygen $2 \mathrm{p}$ orbital and put it on the copper site. In cuprates, x-ray absorption spectroscopy has shown that this is the smallest charge excitation gap with value, $\Delta \sim$ $2-4 \mathrm{eV}$, which is smaller than $U_{d}[15-17]$. The consequence of this is that when holes are first doped into the $\mathrm{CuO}_{2}$ plane, it is into the oxygen $2 \mathrm{p}$ orbitals.

\subsubsection{Effective Models of the $\mathrm{CuO}_{2}$ Plane}

An effective model describing the essential physics of the $\mathrm{CuO}_{2}$ plane is the three-band Hubbard model with Hamiltonian [18],

$$
\begin{aligned}
\hat{H}=-t_{d p} & \sum_{\langle i, j\rangle \sigma}\left(\hat{d}_{i \sigma}^{\dagger} \hat{p}_{j \sigma}+h . c\right)-t_{p} \sum_{\left\langle j, j^{\prime}\right\rangle \sigma}\left(\hat{p}_{j \sigma}^{\dagger} \hat{p}_{j^{\prime} \sigma}+h . c\right)+t_{p}^{\prime} \sum_{\left\langle j, j^{\prime \prime}\right\rangle \sigma}\left(\hat{p}_{j \sigma}^{\dagger} \hat{p}_{j^{\prime \prime} \sigma}+h . c\right) \\
& +\Delta \sum_{j \sigma} \hat{n}_{p, j \sigma}+U_{d} \sum_{i} \hat{n}_{d, i \uparrow} \hat{n}_{d, i \downarrow}+U_{p} \sum_{i} \hat{n}_{p, j \uparrow} \hat{n}_{p, j \downarrow}+V \sum_{\langle i, j\rangle} \hat{n}_{d, i} \hat{n}_{p, j}, \quad
\end{aligned}
$$

where $\hat{d}_{i \sigma}$ annihilates a hole with spin $\sigma$ on the $\mathrm{Cu} 3 \mathrm{~d}_{x^{2}-y^{2}}$ orbital at site $i$, and $\hat{p}_{j \sigma}$ is that on the $\mathrm{O} 2 \mathrm{p}$ orbital at site $j$. The number operators are given by $\hat{n}_{d, i \sigma}=\hat{d}_{i \sigma}^{\dagger} \hat{d}_{i \sigma}$ and $\hat{n}_{p, j \sigma}=\hat{p}_{j \sigma}^{\dagger} \hat{p}_{j \sigma}$. The summation $\langle i, j\rangle$ represents that over nearest-neighbour $\mathrm{Cu}-\mathrm{O}$ bonds while the sum over $\left\langle j, j^{\prime}\right\rangle$ and $\left\langle j, j^{\prime \prime}\right\rangle$ are for nearest and next-nearest-neighbour O-O bonds as shown in figure $1.3(\mathrm{~b}) . U_{p}$ is an onsite Coulomb repulsion for the oxygen 2 p orbitals and $V$ is an inter-site repulsion between electrons on nearest-neighbour $\mathrm{Cu}$ and $\mathrm{O}$ sites.

Typically however, the theoretical starting point for understanding of cuprate physics is that of the single band Hubbard or $t-J$ model. Zhang and Rice justified the potential applicability of these single-band models through the insight that a hole doped onto into the $\mathrm{CuO}_{2}$ plane might delocalise over the 4 oxygens surrounding it and form a Kondo like singlet with the hole on the $\mathrm{Cu}$ site; known as a Zhang-Rice singlet [19]. This singlet state can then hop through the lattice as if it were a hole in a background of localised spins on $\mathrm{Cu}$ sites with an anti-ferromagnetic coupling. Thus a low-energy effective description of the three band Hubbard model is plausibly given by the $t-J$ 
Hamiltonian,

$$
\hat{H}=-t \sum_{\langle i, j\rangle \sigma} P_{G}\left(\hat{c}_{i \sigma}^{\dagger} \hat{c}_{j \sigma}+h . c\right) P_{G}+J \sum_{\langle i, j}\left(\hat{\vec{S}}_{i} \cdot \hat{\vec{S}}_{i}-\frac{1}{2} \hat{n}_{i} \hat{n}_{j}\right)
$$

where $c_{i, \sigma}$ annhiliates a hole on site $i$ with spin $\sigma, \hat{\vec{S}}_{i}$ is the spin operator and $P_{G}$ is the Gutzwiller projection operator that projects out doubly occupancy of any site.

It is well known that this $t-J$ Hamiltonian can be obtained from the single band Hubbard Hamiltonian,

$$
\hat{H}=-t \sum_{\langle i, j\rangle \sigma}\left(\hat{c}_{i \sigma}^{\dagger} \hat{c}_{j \sigma}+h . c\right)+U \sum_{i} \hat{n}_{i \uparrow} \hat{n}_{i \downarrow}
$$

by pertubatively intergrating out high energy degrees of freedom in the the limit $U / t>>$ 1 [20], shrinking the relevant Hilbert space and leading to a more practically soluble model. Thus, the physics of the $\mathrm{CuO}_{2}$ plane is often approached from one of these single-band models. However, as we will see in chapter 4 this potentially misses some key aspects of cuprate phenomenology.

\subsubsection{Phase Diagram}

A schematic phase diagram of hole-doped cuprates is shown in figure 1.5. The most welldefined features of the phase diagram are an insulator that becomes anti-ferromagnetic for $T<T_{N}$ at small hole concentration, $p$, and a dome of high temperature superconductivity with highest transition temperature at $\mathrm{p} \sim 17 \%$. There is also a region of suppressed spectral weight at the Fermi energy known as the the pseudogap regime and a region of anomalous normal state transport above the superconducting dome at optimal doping, often called the strange metal. These aspects of the phase diagram will be discussed further below. In section $1.4 \mathrm{I}$ will go on to discuss the focus of this thesis: the density wave order which has recently been discovered as ubiquitous in under-doped cuprates. 


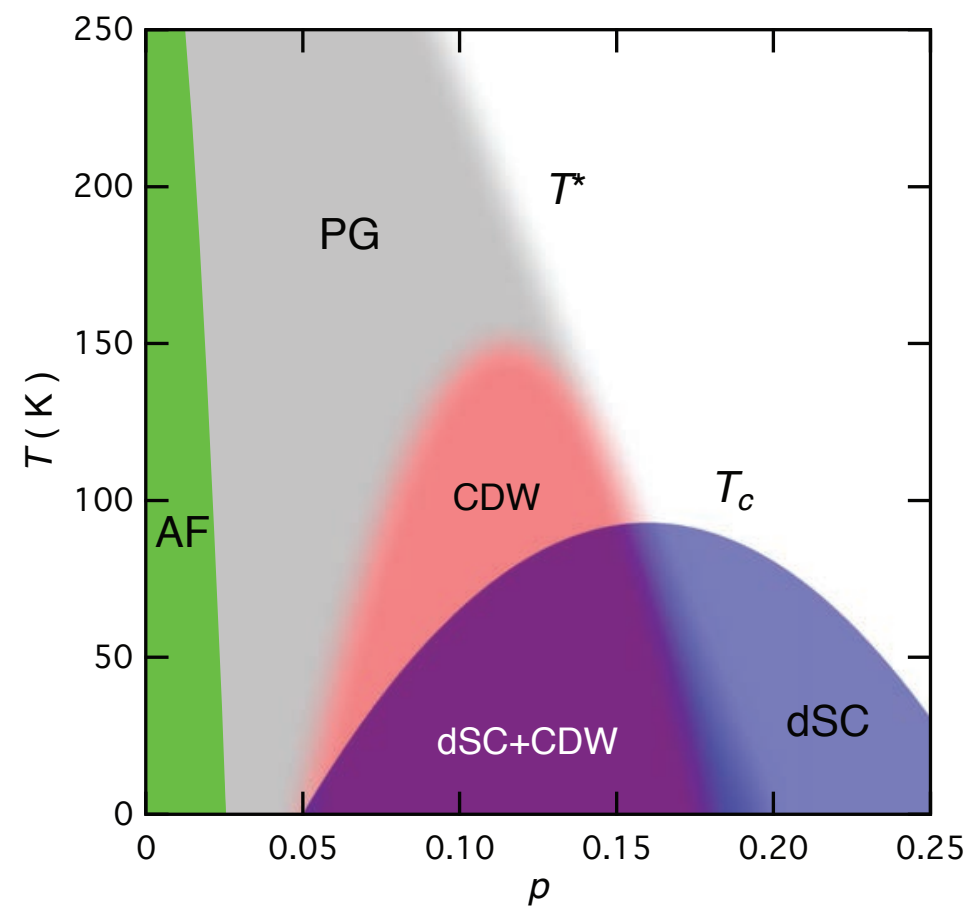

Figure 1.5: Schematic hole-concentration - temperature phase diagram of cuprate superconductors. The green region labelled AF represents an anti-ferromagnetic insulating state. The blue region labelled dSC is a dome of high temperature $d$-wave superconductivity with critical temperature $T_{c}$. The grey shaded region denotes that pseudogap regime, with characteristic temperature $\mathrm{T}^{*}$, terminating at hole-doping, $p \sim$

0.19 holes per $\mathrm{Cu}$. The pink area is a dome of charge density wave order.

\subsubsection{Anti-Ferromagnetism}

The anti-ferromagnetism of the undoped cuprates arises from the Anderson superexchange mechanism, where virtual hopping of electrons between $\mathrm{Cu} 3 \mathrm{~d}_{x^{2}-y^{2}}$ through the $\mathrm{O} 2 \mathrm{p}$ orbitals lowers the energy if the spins of the electrons localised in $3 \mathrm{~d}_{x^{2}-y^{2}}$ orbitals are anti-aligned [20]. This leads to Néel type anti-ferromagnetic order with a critical temperature $T_{N}$. Doping of holes into the $\mathrm{O} 2 \mathrm{p}$ orbitals frustrates this antiferromagnetic coupling and leads to a rapid suppression of $\mathrm{T}_{N}$ with hole doping.

\subsubsection{High-Temperature $d$-Wave Superconductivity}

The superconductivity in cuprates in unconventional in several senses. The first and most striking of these are superconducting critical temperatures as high as $165 \mathrm{~K}$ [21]. To 
learn how such high transition temperatures arise and how we might extend this towards room temperature is the aspiration behind research into cuprate superconductors.

Cuprate superconductivity is also unconventional in a more well defined sense: its pairing symmetry. In the conventional superconductors discussed in section 1.1.2 the pairing amplitude transforms like the trivial representation of the point group (s-wave). In cuprates it is well established that the pairing amplitude is $d_{x^{2}-y^{2}}$ like [11]. It changes sign under $90^{\circ}$ rotations, leading to four line nodes in the gap function along the $(1,1)$ directions. In a tetragonal lattice, the pairing amplitude would transform like the $\mathrm{B}_{1 g}$ representation of the point group, breaking lattice rotation symmetry.

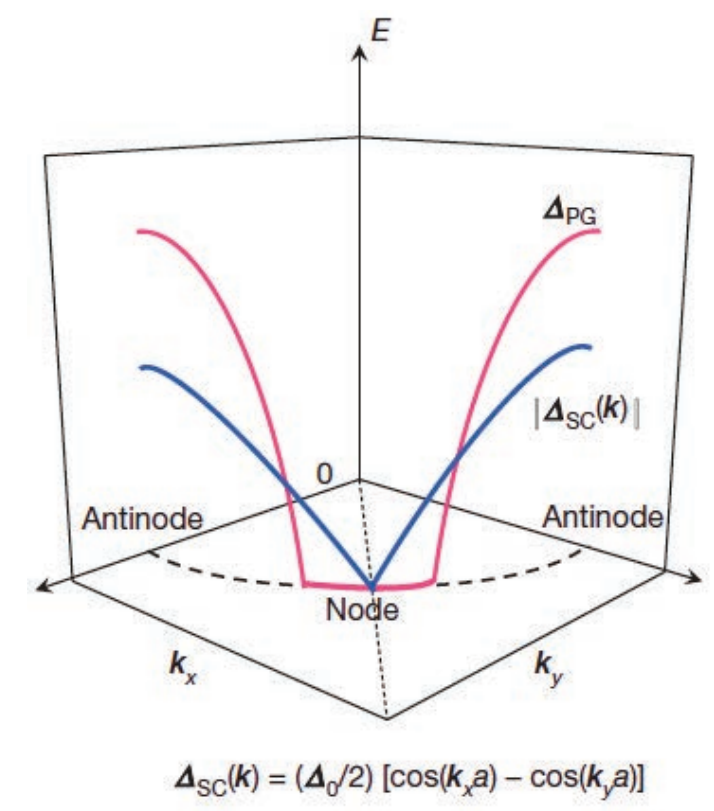

FIGURE 1.6: The form of the superconducting gap $\left(\Delta_{S C}\right)$ and pseudogap in momentum space. The $d_{x^{2}-y^{2}}$ like superconducting gap (blue) has nodes along the $\left(k_{x}, k_{y}\right)=(1,1)$ directions whereas in the pseudogap regime $\left(T_{c}<T<T^{8}\right)$ there are arcs of zero-energy excitations (shown in red). Reproduced from reference [22] with permission.

\subsubsection{Strange Metal}

Above the superconducting dome at optimal doping there is a region of strange metallic behaviour characterised by T-linear resistivity persisting up to the highest temperatures measured. Furthermore, the magnitude of the resistivity is such that if one were to infer a mean free path for current carrying quasi-particles, it would be less than their de-Broglie wavelength, seemingly invalidating the quasi-particle picture. 
This, and similar behaviour seen in other materials [23], thus far defies a coherent theoretical understanding and represents one of the greatest outstanding challenges in our understanding of the cuprates.

\subsubsection{The Pseudogap}

The hole doped cuprate phase diagram harbours a region which exhibits a pseudogap, i.e a partial suppression of spectral weight at the Fermi energy for $T_{c}<T<T^{*}$. This region is indicated by the grey shaded region labelled PG in figure 1.5. The existence of such a suppression has been indicated by a large number of experimental techniques $[22]$.

Angular Resolved Photoemission Spectroscopy (ARPES) has given particular insight into how the pseudogap forms in momentum space [24, 25]. For $T_{c}<T<T^{*}$ there are coherent quasi-particle peaks in a narrow region of momenta near the zone-diagonals (nodal regions). In contrast, the anti-nodal region of the Brillouin zone exhibits a suppression of low energy spectral weight over the pseudogap energy scale and an absence of any quasi-particle peaks. This is illustrated in figure 1.6. With increasing doping the coherent region grows and eventually forms a full Fermi surface of coherent quasiparticles.

STM of underdoped cuprates also exhibits a pseudogap in tunnelling spectroscopy measurements [26-28]. This is characterised by a suppression of tunnelling conductance near the chemical potential over a range of bias voltages $\Delta_{0} / e$, the magnitude and doping dependence of $\Delta_{0}$ being consistent with other measures of the pseudogap energy scale.

A lack of coherence in the anti-nodal regions of the Brillouin zone is also detected by STM quasi-particle interference measurements, albeit for $T<T_{c}$. In these measurements, modulations in the tunnelling conductance caused by scattering interference of Bogoliubov quasi-particles are not observed for states beyond the anti-ferromagnetic zone boundary in momentum space for $p<0.19$. For $p>0.19$, interference patters arising from Bogoliubov quasi-particles extending out to the full Brillouin zone boundary are observed $[29,30]$. The precise relationship between this and the Fermi arcs observed by ARPES is not clear, because for $T<T_{c}$ ARPES indicates coherent quasi-particles in the anti-nodal region for all dopings within the superconducting dome [31]. 
Measurements indicating the existence of a plethora of broken symmetries in the pseudogap regime have been reported, including time-reversal, lattice-translational, pointgroup, spin-rotation and time-reversal symmetry-breaking [32-37]. Of central interest in this thesis are those that break lattice translational symmetry. I will now go on to discuss translational symmetry breaking as well as its discovery and phenomenology in cuprates.

\subsection{Charge Density Waves}

A charge density wave is a state of matter that breaks lattice translational symmetry whilst preserving time-reversal, spin rotation and phase symmetries. In such a state any density-like observable, i.e one that is invariant under time-reversal, spin-rotation and phase-rotation operations, will be modulated with a periodicity different to that of the lattice. One such quantity is charge density, which gives its name to this state of matter [38].

For the square lattice of the $\mathrm{CuO}_{2}$ plane we can expand the charge density wave in order parameters as follows:

$$
\begin{aligned}
& \left\langle\hat{\psi}_{\sigma}^{\dagger}\left(\vec{r}_{1}\right) \hat{\psi}_{\sigma}\left(\vec{r}_{2}\right)\right\rangle= \\
& F\left(\vec{r}_{1}-\vec{r}_{2}\right)\left[\rho_{\vec{Q}_{x}}(\vec{R}) e^{i \vec{Q}_{x} \cdot \vec{R}}+\rho_{-\vec{Q}_{x}}(\vec{R}) e^{-i \vec{Q}_{x} \cdot \vec{R}}+\rho_{\vec{Q}_{y}}(\vec{R}) e^{i \vec{Q}_{y} \cdot \vec{R}}+\rho_{-\vec{Q}_{y}}(\vec{R}) e^{-i \vec{Q}_{y} \cdot \vec{R}}\right]+\ldots
\end{aligned}
$$

where $\vec{R}=\left(\vec{r}_{1}+\vec{r}_{2}\right) / 2$ and $\rho_{\vec{Q}_{x}}, \rho_{-\vec{Q}_{x}}, \rho_{\vec{Q}_{y}}$ and $\rho_{-\vec{Q}_{y}}$ are charge density wave (CDW) order parameters. They are complex scalar fields that carry momenta $\vec{Q}_{ \pm x}$ and $\vec{Q}_{ \pm y}$ running along orthogonal directions $\vec{x}$ and $\vec{y}$. A constraint $\rho_{\vec{Q}_{x}}=\rho_{-\vec{Q}_{x}}^{*}$ ensures that the charge density is real. For $\vec{Q}_{x, y}$ that are incommensurate with the lattice, each order parameter spontaneously breaks a U(1) symmetry associated with the spatial phase of the density wave. If $\vec{Q}$ is commensurate with the lattice, the spontaneously broken symmetry becomes discrete. The function $F\left(\vec{r}_{1}-\vec{r}_{2}\right)$ encodes the local rotational symmetry of the charge density wave or form factor.

In the above I discussed how superconductivity is a particle-particle instability or an instability to forming pairs. In the superconducting state there is a non-zero anomalous 
expectation $\left\langle\hat{c}_{\sigma, \vec{k}}^{\dagger} \hat{c}_{-\sigma,-\vec{k}}^{\dagger}\right\rangle$. The CDW however is a particle-hole instability with a nonzero value of $\left\langle\hat{c}_{\sigma, \vec{k}}^{\dagger} \hat{c}_{\sigma, \vec{k}+\vec{Q}}\right\rangle$. The best known example of such an instability is the Peierls transition [39].

Following the steps we took in section 1.1.2, let us examine the RPA particle-hole susceptibility

$$
\chi_{p h}(\vec{q}, \omega)=\frac{\chi_{p h}^{0}(\vec{q}, \omega)}{1+g \chi_{p h}^{0}(\vec{q}, \omega)},
$$

where

$$
\chi_{p h}^{0}(\vec{q}, \omega)=\frac{1}{\Omega} \sum_{\vec{k}} \frac{f\left(\epsilon_{\sigma, \vec{k}}\right)-f\left(-\epsilon_{\sigma, \vec{k}+\vec{Q}}\right)}{\hbar \omega+\epsilon_{\sigma, \vec{k}}-\epsilon_{\sigma, \vec{k}+\vec{q}}+i 0^{+}} .
$$

The bare (non-interacting) susceptibility for $\chi_{p h}^{0}(\vec{q}, \omega)$ is shown in figure 1.7 for a free Fermi-gas is dimensions $\mathrm{d}=1,2$ and 3 at $T=0$. In $\mathrm{d}=1$ this function diverges at a wave-vector $\vec{q}=2 \vec{k}_{F}$ that connects points on opposite sides of the Fermi surface. This divergence arises because $\epsilon_{\sigma, \vec{k}}=\epsilon_{\sigma, \vec{k}+\vec{q}}$ over a finite domain in $\mathrm{d}=1$. From the denominator of equation 1.25 we can see that this will lead to a divergence in $\chi_{p h}^{0}(\vec{q}, \omega)$, signaling an instability towards CDW order. This property that $\epsilon_{\sigma, \vec{k}}=\epsilon_{\sigma, \vec{k}+\vec{Q}}$ over a finite domain is referred to as perfect nesting.

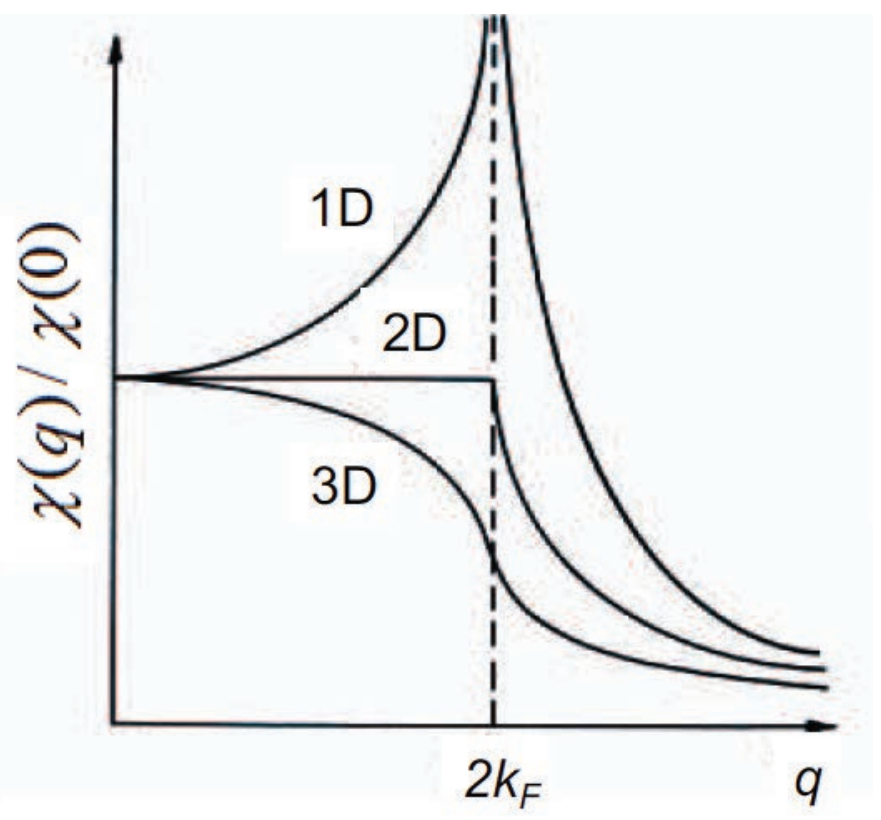

Figure 1.7: Bare particle-hole susceptibility, $\chi(q)=\chi_{p h}^{0}(|\vec{q}|, \omega=0)$ for Fermi gas in dimension $\mathrm{d}=1,2 \& 3$ at $T=0$. The logarithmic divergence at $q=2 k_{F}$ in $\mathrm{d}=1$ signifies a weak coupling instability to CDW order: the Peierls instability. In $d>1$ the susceptibility is not divergent and there is no weak-coupling instability of the Fermi liquid to CDW. 
Peierls recognised that, due to this perfect nesting, a one-dimensional metal would always be unstable to distorting the lattice at wave-vector $\vec{Q}=2 \vec{k}_{F}$ [39]. The decrease in internal energy from opening a gap at the Fermi surface will always out-weigh the increase due to the lattice distortion. This weak-coupling instability (it exists for arbitrarily small repulsive interactions) is known as the Peierls instability.

In $d>1$ the story is quite different. The perfect nesting condition is not fulfilled except in finely tuned circumstances. Consequently there is no divergence of $\chi_{p h}^{0}(\vec{q}, \omega)$ (as show for the free Fermi gas in figure 1.7) and no weak-coupling instability to CDW order, except under finely tuned conditions. This is to be contrasted with the particle-particle susceptibility which always diverges because, in the presence of time-reversal symmetry, $\epsilon_{\sigma, \vec{k}}=\epsilon_{-\sigma,-\vec{k}}$. This can be viewed as another type of perfect nesting which persists to arbitrary dimension. Thus the BCS superconducting instability is, in general, the only weak-coupling instability of the Fermi liquid.

The cuprate superconductors are quasi two-dimensional. For realistic Fermi surfaces, any CDW order in these materials requires a critical effective interaction strength between quasi-particles. Consequently the ordering temperature will depend on the strength of the effective interactions and the wave-vector will be influenced by any momentum dependence of the interaction. Extending equation 1.24 to an interaction with momentum dependence, a rough criterion for forming density wave order at wave-vector $\vec{Q}$ is that $g(\vec{q}) \chi_{p h}^{0}(\vec{q}, 0)=1$. In considering a mechanism for the formation of CDW in cuprates, it is important to keep in mind the importance of both the the Fermi surface (if one exists) and the interaction between its quasi-particles.

\subsection{Unconventional Density Waves in Cuprates}

CDW order has now been detected in several hole-doped cuprate compounds and would thus appear to be ubiquitous in cuprates. This in turn suggests that it is an intrinsic and generic property of doping holes into the $\mathrm{CuO}_{2}$ plane. However, its relationship to the high-temperature superconductivity and pseudogap remains unclear.

The first detection of CDW in cuprates was made in a family of cuprates based on the insulator $\mathrm{La}_{2} \mathrm{CuO}_{4}$. In $\mathrm{La}_{2-x-y} \mathrm{Nd}_{y} \mathrm{Sr}_{x} \mathrm{CuO}_{4+\delta}$ and $\mathrm{La}_{2-x} \mathrm{Ba}_{x} \mathrm{CuO}_{4+\delta}$, "stripe" order, where co-linear anti-ferromagnetic spin and charge modulations $\vec{Q}_{s}$ and $\vec{Q}_{c}$ exist with 
the relationship $\overrightarrow{Q_{c}}=2 \vec{Q}_{s} \approx\left(\frac{1}{4}, 0\right) ;\left(0, \frac{1}{4}\right)$, was detected by neutron scattering [40]. The stripe order is strongest in the vicinity of hole-doping $p \approx 1 / 8$ where there is an anomalous suppression in the bulk superconducting $T_{c}$, known as the " $1 / 8$ th anomaly" [41]. The resonant x-ray scattering study of Abbamonte et al. characterised this stripe order as the segregation of holes in the $\mathrm{CuO}_{2}$ plane into regions of undoped antiferromagnetic insulator separated by domain walls of holes [42].

Short-ranged $\mathrm{CDW}$ order was first detected in $\mathrm{Bi}_{2} \mathrm{Sr}_{2} \mathrm{CaCu}_{2} \mathrm{O}_{8+\delta}$, $\mathrm{Bi}_{2-y} \mathrm{~Pb}_{y} \mathrm{Sr}_{2-x} \mathrm{La}_{x} \mathrm{CuO}_{6+\delta}$ and $\mathrm{Na}_{x} \mathrm{Ca}_{2-x} \mathrm{CuO}_{2} \mathrm{Cl}_{2}$ by scanning tunnelling microscopy [43-46]. Advances in resonant x-ray scattering then led to the detection of short-ranged $\mathrm{CDW}$ order in $\mathrm{YBa}_{2} \mathrm{Cu}_{3} \mathrm{O}_{6+x}$, which was subsequently also detected by hard x-ray diffraction [47-49]. Resonant x-ray scattering also confirmed the presence of CDWs in the bulk of $\mathrm{Bi}_{2} \mathrm{Sr}_{2} \mathrm{CaCu}_{2} \mathrm{O}_{8+\delta}$ and $\mathrm{Bi}_{2-y} \mathrm{~Pb}_{y} \mathrm{Sr}_{2-x} \mathrm{La}_{x} \mathrm{CuO}_{6+\delta}$ with the same wave-vectors as those detected by STM studies, as well as detecting short-range CDW order in $\mathrm{HgBa}_{2} \mathrm{CuO}_{4+\delta}$ [50].

The first hint of $\mathrm{CDW}$ order in $\mathrm{YBa}_{2} \mathrm{Cu}_{3} \mathrm{O}_{6+x}$ was given by the observation of quantum oscillations in $\mathrm{YBa}_{2} \mathrm{Cu}_{3} \mathrm{O}_{6+x}$ whose frequencies correspond to small electron pockets [51]. Quantum oscillations, also implying the existence of small electron pockets, were subsequently detected in Hg-1012 [52]. A reconstruction of the Fermi surface by a CDW was proposed as an explanation of these observations [53].

Developments in high magnetic field x-ray diffraction techniques are providing exciting new information about the cuprate CDW [54-56]. Pulsed field measurements utilising a free electron laser have detected the onset of a $\mathrm{CDW}$ in $\mathrm{YBa}_{2} \mathrm{Cu}_{3} \mathrm{O}_{6+x}$ in applied magnetic fields $\gtrsim 15 \mathrm{~T}$, with a correlation volume of $\sim 10^{5}$ unit cells. This high-field CDW, which I will call CDW-II, appears distinct from the short-ranged charge order present at $\mathrm{B}=0$, in that it is coherent along the direction perpendicular to the $\mathrm{CuO}_{2}$ planes with a periodicity of 1 unit cell. In contrast, the zero-field CDW shows very weak correlations along this direction, with a periodicity of $\approx 2$ unit cells.

These high-field x-ray diffraction measurements also find that CDW-II is uni-directional, with modulations along only one of the $\mathrm{Cu}-\mathrm{O}$ directions $[55,56]$. This is in agreement with earlier high magnetic field NMR measurements showing a splitting of ${ }^{63} \mathrm{Cu}$ lines for $\mathrm{B}>28.5 \mathrm{~T}$, consistent with a uni-directional modulation of charge density along a $\mathrm{Cu}-\mathrm{O}$ direction [57]. 
Details of the experimental evidence for CDW order in cuprates is given in the appropriate chapters throughout this thesis. A recent review can also be found in reference $[58]$.

\subsection{The Significance of CDW in Cuprates}

Although they differ slightly in detail, the CDWs observed in the cuprates have several generic features. The wave-vector(s) are axial, meaning that they are along the $\mathrm{Cu}-\mathrm{O}$ directions $\vec{Q}_{x}=(Q, 0)$ and $\vec{Q}_{y}=(0, Q)$. At zero magnetic field the CDW order is short-ranged with correlation length no longer than 2 wavelengths, except in the case of $\mathrm{La}_{2-x} \mathrm{Ba}_{2} \mathrm{CuO}_{4+\delta}[58]$.

The CDW onset temperature forms a dome centered on $p=0.12$ as shown in figure 1.5. At this doping there is an anomaly in $T_{c}(p)$ so that it either forms a local minimum or a point of inflection, with $T_{c}$ in some materials being suppressed to very low temperatures [59]. In others the effect is weaker at zero field, but the application of a magnetic field suppresses $T_{c}$ to zero at this doping, splitting the superconducting dome in two [60]. This already suggests an intimate relationship between the CDW and superconductivity.

In 2002 Hoffman et al., using SI-STM, discovered halos of periodic conductance modulations surrounding superconducting vortex cores in $\mathrm{Bi}_{2} \mathrm{Sr}_{2} \mathrm{CaCu}_{2} \mathrm{O}_{8+\delta}$ [45]. These modulations, shown in figure 1.8 (a), are indicative of CDW order enhanced by the suppression of superconductivity.

Further evidence of a strong relationship between between superconductivity and CDW order is seen by x-ray scattering studies in a magnetic field. Figure 1.8 (b) shows data from Chang et al on $\mathrm{YBa}_{2} \mathrm{Cu}_{3} \mathrm{O}_{6+x}$ [49]. At zero magnetic field the scattering intensity at the CDW wave-vector increases gradually with decreasing temperature, reaching a maximum at $T_{c}$ and then decreasing again. The application of a magnetic field has no effect on the scattering intensity above $T_{c}$ but the intensity rapidly increases with magnetic field below $T_{c}$. This is suggestive of a strong competition between CDW formation and superconductivity.

The most basic but fundamental question that needs to be addressed is what set of symmetries does the cuprate CDW break? To answer this question is to establish what 

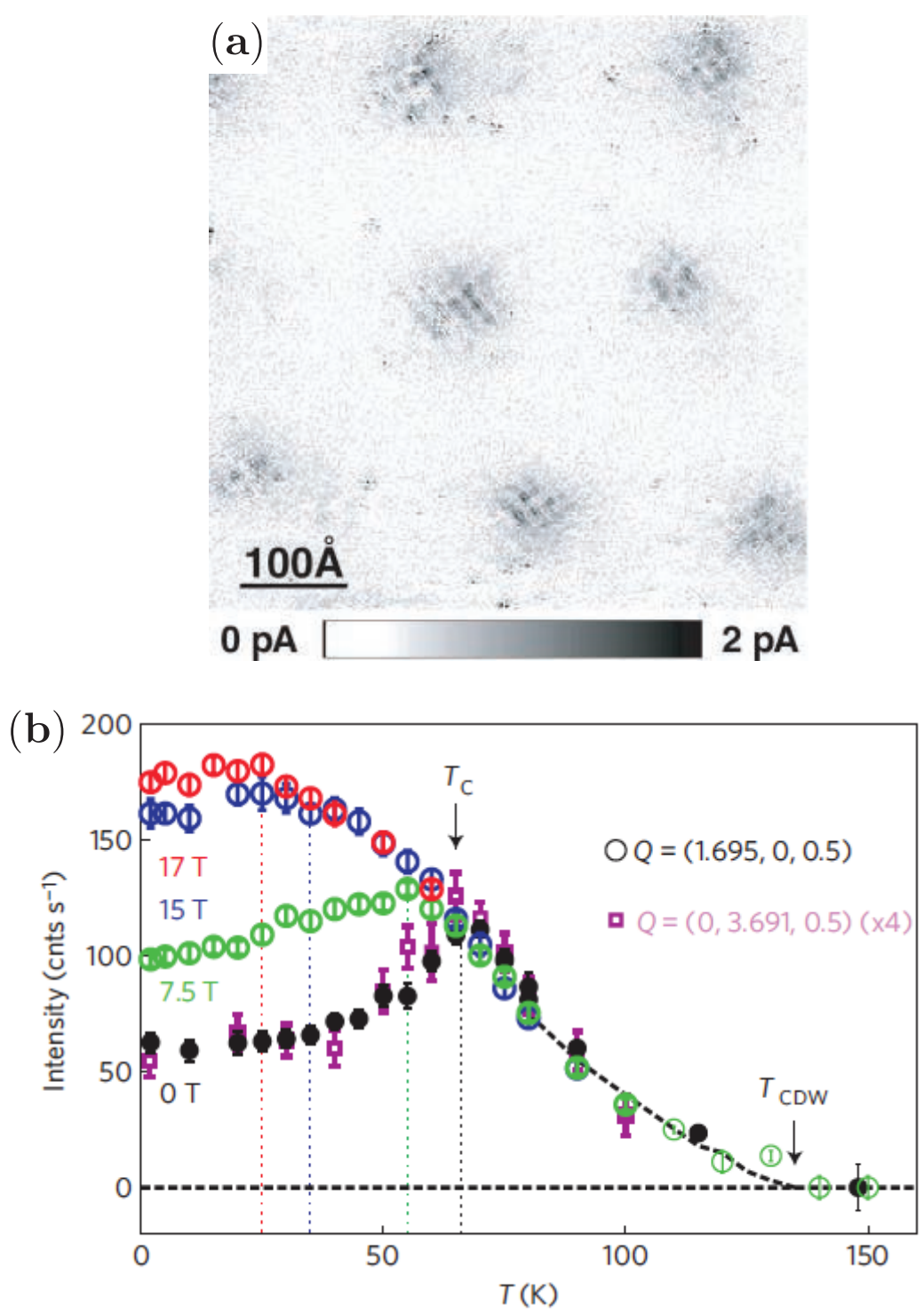

Figure 1.8: (a) Approximately 4a $\mathrm{a}_{0}$ periodic tunnelling conductance modulations surrounding superconducting vortex cores in $\mathrm{Bi}_{2} \mathrm{Sr}_{2} \mathrm{CaCu}_{2} \mathrm{O}_{8+\delta}$, measured using SI-STM. These suggest halos of enhanced CDW order forming due to the suppression of superconductivity. Reproduced from reference [45] with permission. (b) Temperature and magnetic field dependence of x-ray diffraction intensity at CDW wave-vectors in $\mathrm{YBa}_{2} \mathrm{Cu}_{3} \mathrm{O}_{6+x}$. With decreasing temperature, the scattering intensity onsets gradually at a temperature $T_{C D W}>T_{c}$ and rises to a maximum at $T_{c}$ for $\mathrm{B}=0 \mathrm{~T}$ and then decreases again. As a magnetic field is applied this decrease in scattering intensity is lessened and for the $\mathrm{B}=15 \& 17 \mathrm{~T}$ data continues to rise through $T_{c}$ and saturate. Reproduced from reference [49] with permission. 
phase of matter it is. It is clear that translational symmetry is broken but what of the point group symmetry? This would be broken in a stripe-like state with CDW along $\vec{Q}_{x}$ or $\vec{Q}_{y}$ but not both.

It is possible that experimental observations of CDW result from a state which breaks both translational and phase symmetry. This would be a superconductor that breaks translational symmetry, akin to that first proposed by Fulde, Ferrel, Larkin and Ovchinnikov but in the absence of an applied magnetic field [61-63]. Certain forms of this "pair density wave" (PDW) order would also result in a CDW. This is because the density of Cooper-pairs, which are charged, is modulated. A PDW would offer a natural explanation of why the superconductivity and CDW are so strongly coupled to each other in cuprates.

The PDW is a state of matter which has never been directly detected. Establishing its existence in cuprate superconductors would be profound, not just within condensed matter physics but also in high-energy physics and astronomy where PDW states have been predicted to exist in dense quark matter [64].

There is still great interest and uncertainty in the nature of the cuprate pseudogap. It is natural to question the relationship between the charge order and the pseudogap. For instance, is the pseudogap caused by the CDW; either as a static or fluctuating entity? If not, then by what mechanism does the CDW form out of the pseudogap liquid? Key insights into this can be gained from examining the spectral function in the CDW and pseudogap regimes of the cuprate phase diagram.

Ultimately, given such a strong coupling between CDW and superconductivity, it is important to establish the relationship between them. Is CDW order a necessary ingredient or consequence of a minimal model that describes high temperature superconductivity in cuprates? The goal of the research described in this thesis was to investigate issues such as this.

\subsection{Organisation of this Thesis}

Chapter 2 describes spectroscopic imaging STM (SI-STM), the experimental technique used throughout the research reported in this thesis. It also details the materials studied. 
Chapter 3 details the extension of SI-STM to image states of matter with non-trivial structure within the unit cell of a material.

In chapter $4 \mathrm{I}$ describe utilising the techniques detailed in chapters 2 and 3 to characterise the CDW in $\mathrm{Bi}_{2} \mathrm{Sr}_{2} \mathrm{CaCu}_{2} \mathrm{O}_{8+\delta}$ and $\mathrm{Na}_{x} \mathrm{Ca}_{2-x} \mathrm{CuO}_{2} \mathrm{Cl}_{2}$. I will show that the CDW in these materials modulates a broken rotational symmetry within each unit cell. I will also present evidence that the $\mathrm{CDW}$ in cuprates has a tendency to break rotational symmetry globally as well as locally. The effects of the CDW on the electronic structure will also be discussed.

In chapter 5 I will outline the theoretical basis and development of nanometer resolution scanned Josephson tunnelling microscopy (SJTM) in cuprates. In chapter 6 I will describe inhomogeneous superconducting states of matter known as "pair density waves", as well as evidence and theoretical proposals for their existence in cuprates. In chapter 7 I will detail the use of SJTM to detect periodically modulated superconductivity in $\mathrm{Bi}_{2} \mathrm{Sr}_{2} \mathrm{CaCu}_{2} \mathrm{O}_{8+\delta}$, the first direct detection of such a state in any superconductor. Combining this with knowledge of the CDW from chapter 4 and other reported measurements, I will discuss the consequences of this for the cuprate phase diagram.

In chapter 8 I will summarise the main results and conclusions of this thesis. I will also propose future experiments that may address any remaining open questions and discuss future directions for SJTM. 


\section{Chapter 2}

\section{Spectroscopic-Imaging STM (SI-STM)}

In this chapter I detail the main experimental technique used in this thesis: Spectroscopic-Imaging Scanning Tunnelling Microscopy (SI-STM). I outline the theoretical basis for its use as a tunnelling spectroscopy of superconductors as well as data analysis techniques specific to cuprate superconductors. I also give details of the cuprate samples studied.

Scanning tunnelling microscopy (STM) is a technique which uses the tunnelling current of electrons between a sharp metal tip and a sample to image its surface [65]. An exponential dependence of the tunnelling current on the distance between tip and sample makes the STM very sensitive to small changes in surface topography, allowing atomic resolution imaging. STM can also act as a spectroscopic tool by using the tunnel junction between tip and sample to carry out tunnelling conductance spectroscopy, revealing information about the electronic states contributing to the tunnelling current. The advantage of performing spectroscopy with an STM is that it can be done with atomic resolution. The theoretical basis for the topographic and spectroscopic capabilities of STM are outlined in this chapter.

STM came to take a prominent role in the study of quantum matter with the advent of spectroscopic imaging STM (SI-STM) [27, 28]. In SI-STM the topographic and spectroscopic abilities of the STM are systematically combined. The sample's surface topography and spectroscopic information are obtained simultaneously across a two-dimensional 
grid of points, or pixels. In doing so, one is essentially visualising the energy-resolved density of states of electrons in the material. In this chapter I will detail the techniques needed to obtain both real and momentum space information about the wave-functions in cuprate superconductors.

The rapid and fairly specialised development of the field means that for completeness I must touch on a fairly large number of sub-topics, some of which are fairly technical; one way to use this chapter might be to back-reference to it from the main results chapters of the thesis rather than try to take in every detail on first reading.

\subsection{Quantum Tunnelling as a Spectroscopy}

The experiments presented in this thesis rely on measuring the tunnelling current of electrons between a sample and a metal electrode across an insulating barrier [66, 67]. The tunnelling process consists of transferring an electron from a many-body state that is localised in the left electrode to another many-body state localised in the right electrode. Of course, by symmetry we expect the reverse process to have an equal rate, resulting in zero net current. However, under external application of a bias such as a current or voltage this symmetry is broken resulting in a net a current.

The variation of the current with the applied bias can yield information about the many-body states involved. Calculating the rates of these tunnelling processes from first principles is not generally possible. In the following section I will describe the tunnelling Hamiltonian formalism that can be used to extract spectroscopic information from the tunnelling current, without exactly solving the tunnelling problem [68, 69].

\subsubsection{Tunnelling Hamiltonian Formalism}

Within the tunnelling Hamiltonian formalism, the total Hamiltonian is given by

$$
\hat{H}=\hat{H}_{L}+\hat{H}_{R}+\hat{H}_{T}
$$

where $\hat{H}_{L}$ and $\hat{H}_{R}$ describe the many-body states in the left and right electrodes separately, in the absence of each other [69]. $\hat{H}_{T}$ contains all additional terms that arise from coupling the electrodes to each other through the tunnel junction. 
An exact treatment of this problem for two interacting many-body states in the right and left electrodes is not generally analytically tractable. Instead, we can treat $\hat{H}_{T}$ perturbatively. Typically we use a tunnelling Hamiltonian of the form,

$$
\hat{H}_{T}=\sum_{i, j}\left(M_{i, j} \hat{c}_{L, i}^{\dagger} \hat{c}_{R, j}+M_{j, i}^{*} \hat{c}_{R, j}^{\dagger} \hat{c}_{L, i}\right)
$$

where $\hat{c}_{L, i}^{\dagger}$ creates an electron in state $i$ on the left electrode and $\hat{c}_{R, j}^{\dagger}$ creates an electron in state $j$ on the right electrode [69]. I will now examine the consequences of this Hamiltonian for various choices of the Hamiltonians $\hat{H}_{L}$ and $\hat{H}_{R}$ corresponding to NormalInsulator-Normal (NIN), Superconductor-Insulator-Normal (SIN) and SuperconductorInsulator-Superconductor (SIS) tunnel junctions.

\subsubsection{Normal - Insulator - Normal (NIN) Tunnelling}

In the case of two normal metal electrodes the Hamiltonians are given by

$$
\begin{gathered}
\hat{H}_{L}=\sum_{\vec{k}} \epsilon_{\vec{k}} \hat{c}_{L, \vec{k}}^{\dagger} \hat{c}_{L, \vec{k}} \\
\hat{H}_{R}=\sum_{\vec{q}} \epsilon_{\vec{q}} \hat{c}_{R, \vec{q}}^{\dagger} \hat{c}_{R, \vec{q}} \\
\hat{H}_{T}=\sum_{\vec{k}, \vec{q}}\left(M_{\vec{k}, \hat{q}^{\dagger}}^{\dagger} \hat{c}_{L, \vec{k}}+M_{\vec{q}, \vec{k}}^{*} \hat{c}_{R, \vec{q}}^{\dagger} \hat{c}_{L, \vec{k}}\right)
\end{gathered}
$$

where $\hat{c}_{L, \vec{k}}^{\dagger}$ creates an electron of crystal momentum $\vec{k}$ in the left electrode with energy $\epsilon_{k}$ measured relative to the Fermi energy. Here, spin indices have been suppressed under the assumption that no spin-flip processes take place in tunnelling.

We can then perturbatively calculate the current of electrons from the left to the right electrode using Fermi's golden rule as

$$
I_{L \rightarrow R}=\frac{2 \pi e}{\hbar} \sum_{\vec{k}, \vec{q}}\left|M_{\vec{k}, \vec{q}}\right|^{2} f_{\vec{k}}\left[1-f_{\vec{q}}\right] \delta\left(\epsilon_{\vec{k}}-\epsilon_{\vec{q}}\right),
$$

where $f_{\vec{k}}$ is the occupation number of the state $\vec{k}$. Similarly, the reverse current is given by

$$
I_{R \rightarrow L}=\frac{2 \pi e}{\hbar} \sum_{\vec{k}, \vec{q}}\left|M_{\vec{k}, \vec{q}}\right|^{2} f_{\vec{q}}\left[1-f_{\vec{k}}\right] \delta\left(\epsilon_{\vec{q}}-\epsilon_{\vec{k}}\right),
$$


with the net current from left to right given by $I=I_{L \rightarrow R}-I_{R \rightarrow L}$.

Under the simplifying assumption that $T=0$ and the matrix elements, $\left|M_{\vec{k}, \vec{q}}\right|=|M|$, are a constant for states within $\mathrm{eV}$ of the chemical potential then this reduces to the form

$$
I=\frac{2 \pi e}{\hbar}|M|^{2} \int_{0}^{e V} n_{L}(\epsilon+e V) n_{R}(\epsilon) d \epsilon,
$$

where $n(\epsilon)$ is the momentum integrated density of states at energy $\epsilon$ [69].

Under a further assumption that we choose a right hand electrode with a constant density of states $n_{R}(E)=n_{R}(0)$, then equation 2.8 becomes

$$
I=\frac{2 \pi e}{\hbar}|M|^{2} n_{R}(0) \int_{0}^{e V} n_{L}(\epsilon+e V) d \epsilon
$$

and $n_{L}(E)$ can be measured directly through the differential tunnelling conductance

$$
\frac{d I}{d V}=\frac{2 \pi e^{2}}{\hbar}|M|^{2} n_{R}(0) n_{L}(e V)
$$

The physical tunnelling processes resulting in these equations are depicted in figure 2.1. Tunnelling may only occur from filled states to empty states. Hence by applying a positive bias we are probing the empty states of the left electrode (or sample) and with a negative bias its filled states.

The assumption of an energy independent matrix element, $\left|M_{\vec{k}, \vec{q}}\right|=|M|$, is not in any sense innocuous. For metals described by the independent-particle pictures used in equations 2.3 and 2.4, Harrison calculated the matrix elements $M_{\vec{k}, \vec{q}}$ within the WKB approximation [71]. The conclusion was that the energy dependence of the matrix elements exactly cancels that of the density of states. This results in featureless, ohmic tunnelling $I(V)$ curves.

On the face of it, this would indicate the we cannot use tunnelling as a spectroscopy on metals. However, if the sample we wish to measure is not a single-band metal, well described by a Fermi liquid, then the cancellation between the density of states and the matrix element does not hold and spectroscopy becomes possible [68, 69, 71]. Specifically, equations 2.9 and 2.10 which we derived for metals of independent electrons, are actually also valid for tunnelling between a superconductor and a normal metal electrode. In the next section I will outline why this is the case. 


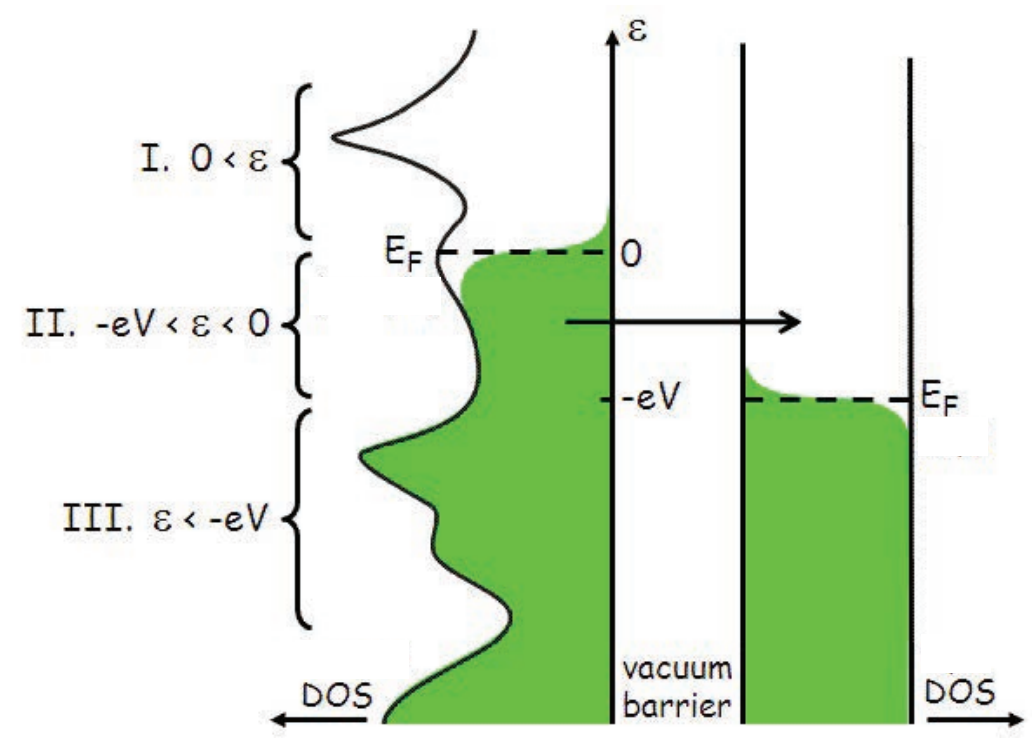

FiguRE 2.1: Schematic of tunnelling from left hand electrode with negative bias applied, to right hand electrode. Green shading indicates filled states. Only energy ranges where left-hand states are filled and right-hand states are empty (i.e region II) contribute to the net tunnelling current. The right hand density of states (DOS) is drawn flat as is required if useful spectroscopic information is to be obtained without deconvolution. Figure adapted from reference [70].

\subsubsection{Superconductor-Insulator-Normal (SIN) Tunnelling}

Now I will discuss tunnelling between a normal metal and a superconductor. This is the configuration of electrodes used in the STM studies of cuprate superconductors in chapter 4, where a tungsten tip is used to perform spectroscopy on cuprate superconductor samples. In this case the normal and superconducting electrode Hamiltonians, $\hat{H}_{N}$ and $\hat{H}_{S}$, are given by

$$
\begin{gathered}
\hat{H}_{N}=\sum_{\vec{q}} \epsilon_{\vec{q}} \hat{c}_{L, \vec{q}}^{\dagger} \hat{c}_{L, \vec{q}} \\
\hat{H}_{S}=\sum_{\vec{k}} E_{\vec{k}}\left(\hat{\alpha}_{\vec{k}}^{\dagger} \hat{\alpha}_{\vec{k}}+\hat{\beta}_{\vec{k}} \hat{\beta}_{\vec{k}}^{\dagger}\right)
\end{gathered}
$$

where

$$
\begin{aligned}
& \alpha_{\vec{k}}=u_{\vec{k}} \hat{c}_{R, \vec{k}, \uparrow}-v_{\vec{k}} \hat{c}_{R,-\vec{k}, \downarrow}^{\dagger} \\
& \beta_{\vec{k}}=u_{\vec{k}} \hat{c}_{R,-\vec{k}, \downarrow}+v_{\vec{k}} \hat{c}_{R, \vec{k}, \uparrow}^{\dagger}
\end{aligned}
$$


and $u_{\vec{k}}$ and $v_{\vec{k}}$ are the BCS coherence factors $[8,69]$.

As presented in chapter 1, the ground state of condensed pairs in the superconducting electrode is described by the BCS wave-function [8]. Its Bogoliubov quasi-particle excitations are a superposition of holes and electrons and are annihilated by the $\alpha_{q}$ and $\beta_{q}$ operators. Their energy spectrum is given by

$$
E_{\vec{k}}=\sqrt{\epsilon_{\vec{k}}^{2}+|\Delta|^{2}}
$$

where $\epsilon_{\vec{k}}$ are the single-particle energies of the normal state band from which the superconducting instability arises and $\Delta$ is the superconducting gap.

In order to consider the current between the electrodes arising from the tunnelling Hamiltonian it is convenient to define new operators which definitely create either an electron or hole in the superconductor, rather than a superposition of the two [72, 73]. These four operators are

$$
\begin{aligned}
& \hat{\alpha}_{e, \vec{k}}^{\dagger}=u_{\vec{k}}^{*} \hat{c}_{\vec{k} \uparrow}^{\dagger}-v_{\vec{k}}^{*} \hat{S}^{\dagger} \hat{c}_{-\vec{k} \downarrow} \\
& \hat{\alpha}_{h, \vec{k}}^{\dagger}=u_{\vec{k}}^{*} \hat{S}_{\vec{k} \uparrow}^{\dagger}-v_{\vec{k}}^{*} \hat{c}_{-\vec{k} \downarrow} \\
& \hat{\beta}_{e, \vec{k}}^{\dagger}=u_{\vec{k}}^{*} \hat{c}_{-\vec{k} \downarrow}^{\dagger}+v_{v}^{*} \hat{S}^{\dagger} \hat{c}_{\vec{k} \uparrow} \\
& \hat{\beta}_{h, \vec{k}}^{\dagger}=u_{\vec{k}}^{*} \hat{S}_{-\vec{k} \downarrow}^{\dagger}+v_{\vec{k}}^{*} \hat{c}_{\vec{k} \uparrow}
\end{aligned}
$$

where $\hat{\alpha}_{e, \vec{k}}^{\dagger}$ creates an electron with wave-vector $\vec{k}$ and $\hat{S}^{\dagger}$ adds a Cooper-pair to the condensate. We can invert these definitions to express the electron creation operator in terms of the new operators, yielding

$$
\hat{c}_{k \uparrow}^{\dagger}=u_{\vec{k}} \hat{\alpha}_{e, \vec{k}}^{\dagger}+v_{\vec{k}}^{*} \hat{\beta}_{h, \vec{k}}
$$

Now consider a term in the tunnelling Hamiltonian $M_{\vec{k}, \vec{q}} \hat{c}_{L, q}^{\dagger} \hat{c}_{R, k}$. Using equation 2.21 this will contribute a term in the current proportional to $\left|u_{\vec{k}}\right|^{2}\left|M_{\vec{k}, \vec{q}}\right|^{2}$ because the second term in equation 2.21 annihilates the BCS ground state. The physical interpretation of the coefficient $\left|u_{\vec{k}}\right|^{2}$ is that it is the probability that a state $\vec{k}$ is not occupied in the BCS wave-function and hence is able to accept an incoming electron. 
The coherence factors $u_{\vec{k}}$ and $v_{\vec{k}}$, which encode details of the superconducting wavefunction, actually drop out of the full expression for the tunnelling current to leave a remarkably simple result. There is another state $\vec{k}^{\prime}$ with $E_{\vec{k}^{\prime}}=E_{\vec{k}}$ but with $\epsilon_{\vec{k}^{\prime}}=$ $-\epsilon_{\vec{k}}$. This will contribute to the current a term $\left|u_{\vec{k}^{\prime}}\right|^{2}\left|M_{\vec{k}^{\prime}, \vec{q}^{\prime}}\right|^{2}=\left|v_{\vec{k}}\right|^{2}\left|M_{\vec{k}^{\prime}, \vec{q}^{\prime}}\right|^{2}$ because $|u(-\epsilon)|=|v(\epsilon)|$. If $\left|M_{\vec{k}^{\prime}, \vec{q}}\right| \approx\left|M_{\vec{k}, \vec{q}}\right|$, since $\vec{k}$ and $\vec{k}^{\prime}$ are near the same point on the Fermi surface then the total contribution from these two terms will be proportional to $\left(\left|u_{\vec{k}}\right|^{2}+\left|v_{\vec{k}}\right|^{2}\right)\left|M_{\vec{k} \vec{q}}\right|^{2}=\left|M_{\vec{k}, \vec{q}}\right|^{2}$ because $\left|u_{\vec{k}}\right|^{2}+\left|v_{\vec{k}}\right|^{2}=1$ as a result of fermionic particle statistics.

At $T=0$ this independence of the coherence factors results in an SIN current

$$
I_{S I N}=\frac{2 \pi e}{\hbar} \sum_{\vec{k}, \vec{q}}\left|M_{\vec{k}, \vec{q}}\right|^{2} \delta\left(E_{\vec{k}}-\left|\epsilon_{\vec{q}}\right|\right) .
$$

From this it is evident that we can treat the tunnelling from a normal metal into a superconductor as if the superconductor were described by independent particles in a semiconductor with density of states

$$
\frac{n_{s}(E)}{n_{s 0}}= \begin{cases}\frac{E}{\sqrt{E^{2}-|\Delta|^{2}}} & E>|\Delta| \\ 0 & E<|\Delta| .\end{cases}
$$

Here $n_{S}(E)$ is the Bogoliubon density of states and $n_{s 0}$ the density of states of the underlying normal band, assumed to be constant. Here I show the result for an $s$-wave superconducting gap. For a $d$-wave gap $n_{S}(E)$ can simply be replaced by the $d$-wave Bogoliubon density of states.

Under the assumption that $\left|M_{\vec{k}, \vec{q}}\right|=|M|$, we arrive at

$$
I_{S I N}=\frac{2 \pi e}{\hbar}|M|^{2} \int_{0}^{e V} n_{s}(\epsilon+e V) n_{n}(\epsilon) d \epsilon,
$$

which is the same form for the tunnelling current derived for a metal of non-interacting quasi-particles derived in section 2.1.2. If we choose a normal metal electrode with a constant density of states $n_{0}$ (such as a tungsten STM tip) then the differential tunnelling conductance

$$
\frac{d I}{d V}=\frac{2 \pi e^{2}}{\hbar}|M|^{2} n_{0} n_{s}(e V)
$$

measures $n_{s}(E)$ directly $[68,69,71,73]$. 


\subsubsection{Superconductor-Insulator-Superconductor (SIS) Tunnelling}

If we only consider the tunnelling of single electrons between two superconducting electrodes then the semiconductor model presented in the previous section still holds due to a similar simplification of the coherence factors [74]. This results in a superconductorinsulator-superconductor tunnelling current

$$
I_{S I S}=\frac{2 \pi e}{\hbar}|M|^{2} \int_{0}^{e V} n_{S 1}(E-e V) n_{S 2}(E) d E
$$

$n_{S 1}(E)$ and $n_{S 2}(E)$ would be given by equation 2.23 with superconducting gaps $\Delta_{1}$ and $\Delta_{2}$ respectively. Neither of the electrodes has a constant density of states near the Fermi energy, resulting in a more complicated expression for the differential tunnelling current [74]:

$$
\frac{d I}{d V}=\frac{2 \pi e^{2}}{\hbar}|M|^{2}\left[n_{S 1}(E) n_{S 2}(E+e V)+\int_{0}^{e V} n_{S 1}(E) \frac{d}{d(e V)} n_{S 2}(E+e V) d E\right] .
$$

This will be used in chapter 5 for the scanned Josephson tunnelling microscope, which uses a superconducting STM tip to measure a superconducting sample.

In addition to the single electron tunnelling processes between superconductors considered here, there is also the possibility of the tunnelling of Cooper-pairs between the electrodes [72]. This is known as the Josephson effect and it will be discussed in chapter 5 , where I will demonstrate the use of Cooper-pair tunnelling to directly visualise the Cooper-pair condensate in $\mathrm{Bi}_{2} \mathrm{Sr}_{2} \mathrm{CaCu}_{2} \mathrm{O}_{8+\delta}$.

Having outlined the theoretical basis for vacuum tunnelling and its use as a spectroscopy I will now go on to describe how it is used in an STM.

\subsection{STM - Principles}

The STM consists of a sharp conducting tip that can be rastered across a conducting sample surface. This is typically achieved by attaching the tip to a piezoelectric scanner tube as shown schematically in figure 2.2 [65]. A bias voltage, $V$, is applied between the two and the vacuum tunnelling current measured. We shall see below that its utility as an extremely sensitive topographic probe derives from the exponential dependence 
of the tunnelling current on the tip-sample distance. The principles of tunnelling spectroscopy detailed in the previous section can also be applied to STM to give access to the atomically resolved local density of states (LDOS).

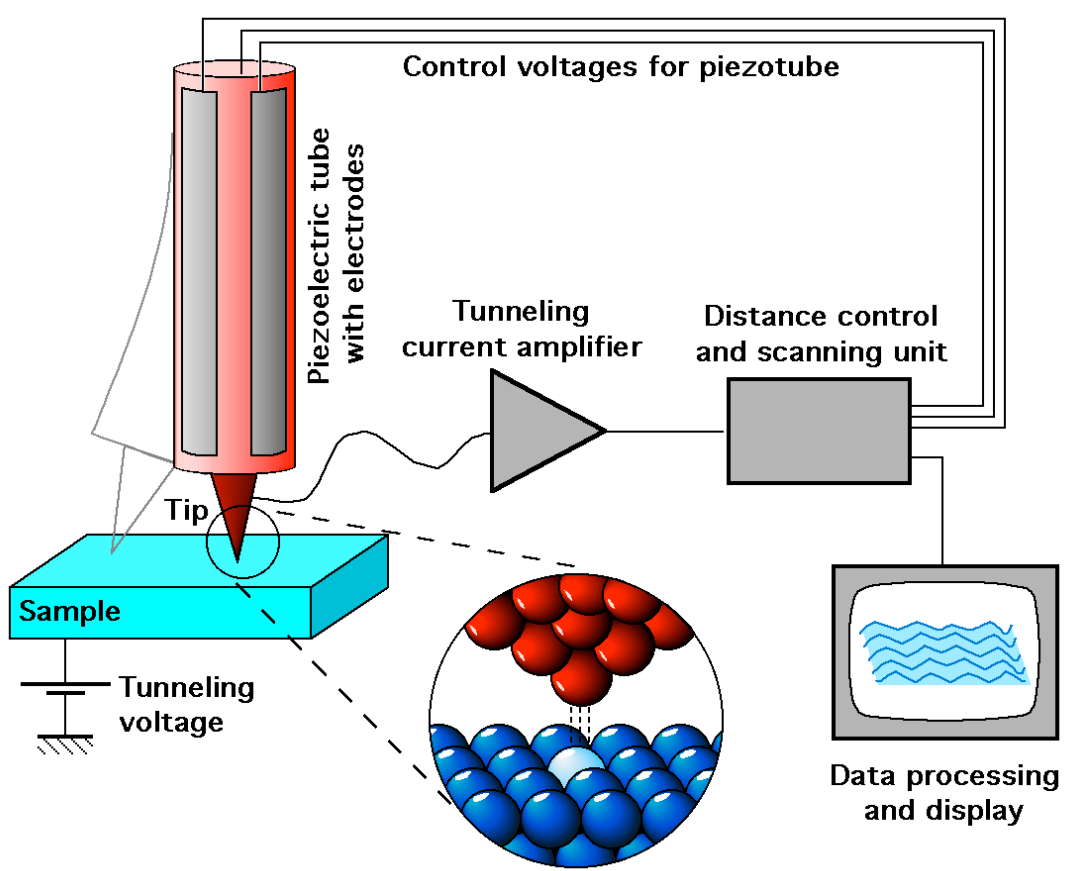

FIgURE 2.2: Schematic of STM operation. This figure illustrates tunnelling between a voltage-biased sample and an atomically sharp tip. In constant current operation the piezoelectric tube is used to scan the tip in the $\mathrm{x}$ and $\mathrm{y}$ directions. As this is done the tunnelling current is measured. A feedback system (PID) is used to adjust $z$ using the piezo-tube so as to keep the current constant. Reproduced from reference [75]

Focussing on the case of SIN tunnelling between a normal metal STM tip and a superconducting sample pertinent to chapter 4 , the elastic tunnelling current between tip and sample under applied bias voltage $V$ at $T=0$ is

$$
I_{S I N}(\vec{r}, z, E=e V)=\frac{2 \pi e}{\hbar} n_{T}(0)|M(\vec{r}, z)|^{2} \int_{0}^{e V} n_{S}(\vec{r}, \epsilon) d \epsilon
$$

$n_{s}(\vec{r}, E)$ is the superconducting LDOS of the sample at position $\vec{r}=(x, y), z$ is the tip-sample separation and energy $E=e V$. The density of states of the STM tip, $n_{T}$, has been taken to be constant here. In the measurements presented in this thesis this was achieved using tungsten STM tips which have a flat density of states near the Fermi energy. Thus, we see that at a given position, $I_{S I N}(\vec{r}, z, V)$ is directly proportional to the energy-integrated density of states. 
The spatially resolved differential tunnelling conductance is given by

$$
g(\vec{r}, z, E=e V)=\frac{2 \pi e^{2}}{\hbar}|M(\vec{r}, z)|^{2} n_{0} n_{S}(\vec{r}, E=e V) .
$$

Thus at a given position, $g(\vec{r}, V)$ is directly proportional to the local density of states $n_{S}(\vec{r}, E)$.

To see why STM is such an effective atomic scale probe we now need to examine the dependence of the matrix elements $|M(\vec{r}, z)|$ on the tip sample distance $z$. Bardeen has shown [68] that if the many-body tip and sample states are known then in principle the matrix elements $\left|M_{\mu \nu}\right|$ can be calculated via

$$
M_{\mu \nu}=\frac{\hbar^{2}}{2 m} \int \mathrm{d} \vec{S} \cdot\left(\psi_{\mu}^{*} \nabla \psi_{n u}-\psi_{\nu} \nabla \psi_{m u}^{*}\right)
$$

where the integral is over any surface lying entirely within the vacuum region. These wave-functions are typically not known but an analytical model that provides much insight was provided by Tersoff and Hamann [76]. They showed that

$$
\left|M_{\mu \nu}\right|^{2} \propto \exp (-2 \kappa z)
$$

where $\kappa=\sqrt{\frac{2 m \phi}{\hbar^{2}}}$ with $\phi$ being the work functions of the tip and sample (which are assumed to be the same), $z$ the tip-sample distance and $m$ the electron mass. $\kappa^{-1}$ represents the maximum decay length of sample wave-functions into the vacuum.

This exponential decay with $z$ is what we might have expected from considering a simple 1D square barrier of height $\phi$ within the WKB approximation. It gives STM an extreme sensitivity to changes in the topographic height of the sample. Below I will discuss how equations 2.28, 2.29 and 2.31 are utilised in the topographic and spectroscopic imaging modalities of STM.

\subsection{STM - Modes of Operation}

\subsubsection{Topography}

To record a topograph a set-point bias $V_{s}$ is applied between tip and sample. A feedback loop is then used to keep $I\left(\vec{r}, Z, e V_{s}\right)$ constant at a set-point value $I_{s}$, by varying $z$, as 
the tip rasters across a sample [77]. In this way one is able to resolve sub-Angström changes in sample topography. An STM topograph, $T(\vec{r})$, consists of an image in which each pixel at position $\vec{r}$ records the value of $z$ required to attain the set-point current.

Let us term $\int_{0}^{e V_{s}} n_{S}(\epsilon, \vec{r}) d \epsilon$ the integrated LDOS. If this and the matrix element are uniform across the sample then the STM "topograph" will be a good approximation to the true topography of the surface.

Figure 2.3 shows an atomically resolved topograph of the $\mathrm{BiO}$ surface of $\mathrm{Bi}_{2} \mathrm{Sr}_{2} \mathrm{CaCu}_{2} \mathrm{O}_{8+\delta}$. Here the atomic contrast is known to result from the $\mathrm{Bi}$ atoms on the insulating surface layer. This layer does not contribute to the low energy LDOS and hence the atomic contrast in figure 2.3 must arise through atomic scale variations in the tunnelling matrix elements.

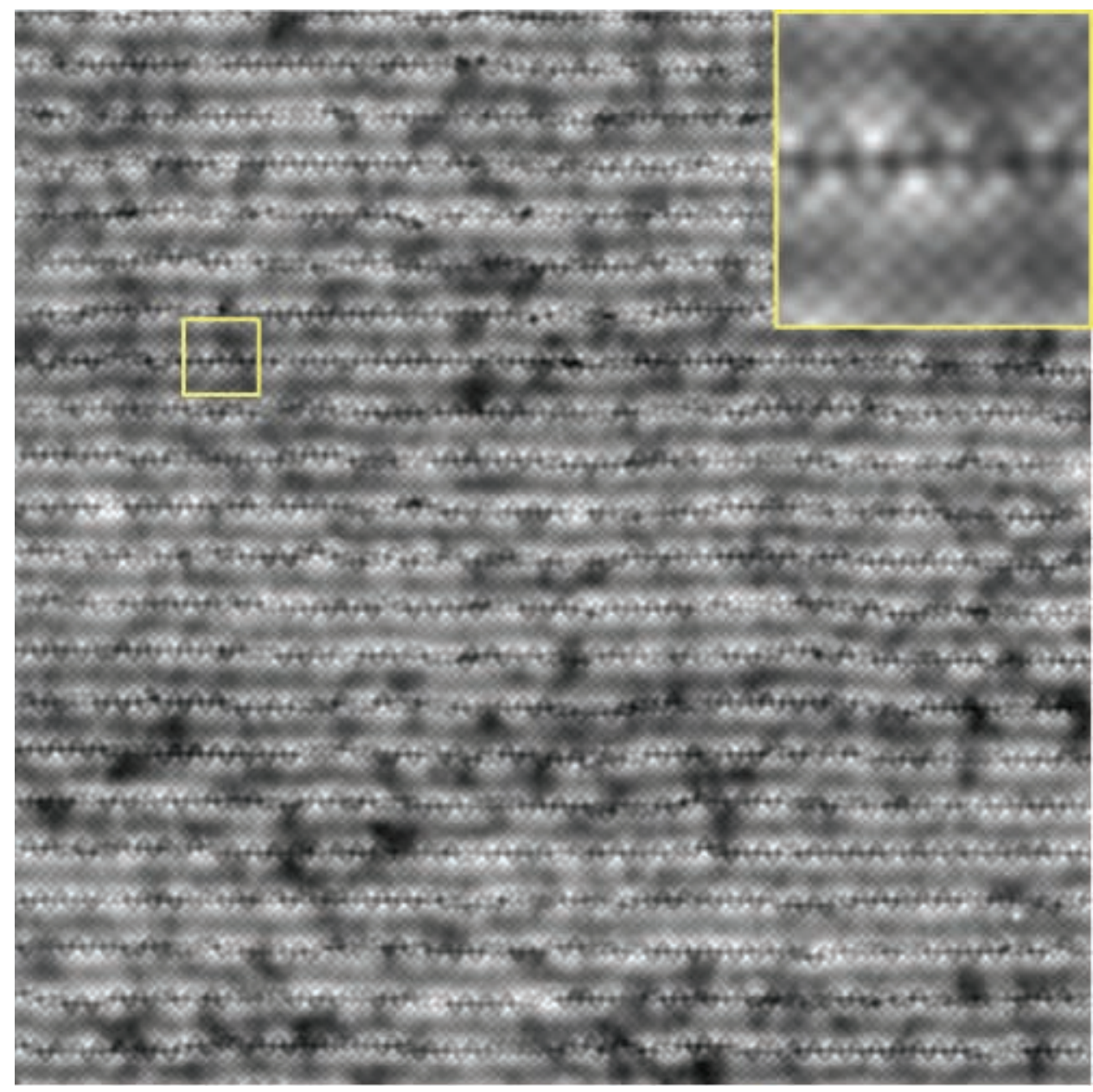

Figure 2.3: Atomically resolved topograph, $T(\vec{r})$, of a $\mathrm{Bi}_{2} \mathrm{Sr}_{2} \mathrm{CaCu}_{2} \mathrm{O}_{8+\delta}$ sample in a $65 \times 65 \mathrm{~nm}$ field of view. Inset is magnified image of the section outlined in yellow. 


\subsubsection{Spectroscopy}

As shown above in equation 2.29, the differential conductance $g(\vec{r}, V)=d I(\vec{r}, z, V) / d V$ at bias voltage $\mathrm{V}$ is proportional to $n_{S}(\vec{r}, e V)$. Thus by holding the tip at constant $z$, sweeping the bias voltage and recording $g(\vec{r}, V)$ one can measure $n_{S}(\vec{r}, e V)$ [77]. While it is possible to measure $I(\vec{r}, z, V)$ and then take a numerical derivative to give $g(\vec{r}, V)$, a considerably better signal to noise ratio can be obtained by using a small $\mathrm{AC}$ modulation of $\mathrm{V}$ from a lock-in amplifier to measure this quantity directly. Let $V(t)=V_{0}+V_{m} \cos (\omega t+\phi)$ then,

$I(\vec{r}, z, V(t))=I\left(\vec{r}, z, V_{0}\right)+\left.\frac{\mathrm{d} I(\vec{r}, z, V)}{\mathrm{d} V}\right|_{V_{0}} V_{m} \cos (\omega t+\phi)+\left.\frac{\left.\mathrm{d}^{2} I(\vec{r}, z, V)\right)}{\mathrm{d} V^{2}}\right|_{V_{0}} V_{m}^{2} \cos ^{2}(\omega t+\phi)+\ldots$,

and hence measurement of the amplitude of the first harmonic gives $g(\vec{r}, z, V)$.

Taking a measurement of this type at a single point on the sample, $\vec{r}$, for a range of DC bias voltages results in a conductance spectrum, proportional to the LDOS, such as that shown in figure 2.4. I will now go on the discuss the systematic collection of these conductance spectra on a fine array of points on the sample to allow an energy resolved visualisation of the electronic structure. This is known as spectroscopic imaging STM (SI-STM).

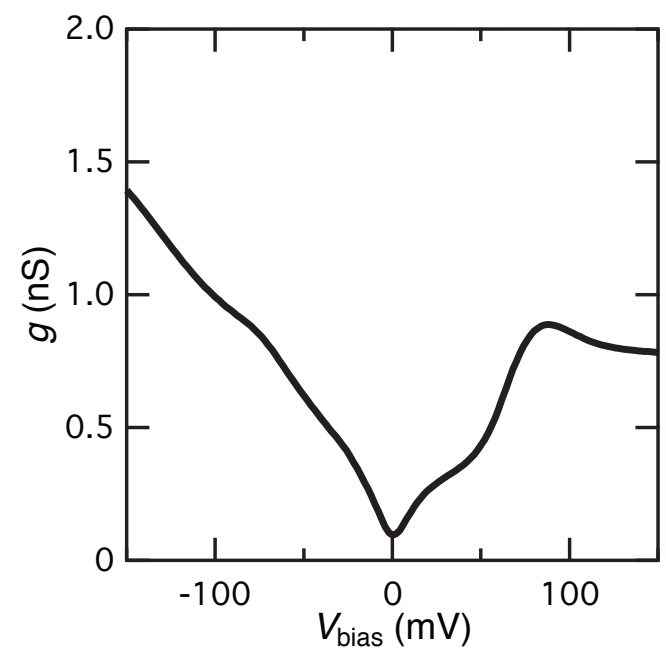

Figure 2.4: Typical differential conductance spectrum, $g(V)$, for a $\mathrm{Bi}_{2} \mathrm{Sr}_{2} \mathrm{CaCu}_{2} \mathrm{O}_{8+\delta}$ sample with $p=6 \%$. 


\subsection{SI-STM}

In spectroscopic imaging STM (SI-STM) current and conductance spectra, $I(\vec{r}, z, E=$ $e V)$ and $g(\vec{r}, z, E=e V)$, are collected on a fine 2D array of points on the sample to provide energy resolved maps of the local density of states and its integral [27, 28]. Simultaneously, a topographic image is also collected so that the spectroscopic maps can be registered to the lattice.

The procedure for collecting data of this type is as follows:

1. Define an regular array of points $\vec{r}_{1}, \vec{r}_{2} \ldots \vec{r}_{N}$ on the surface of the sample where measurements will be made. Each of these points will form a pixel in the resulting spectroscopic maps and simultaneous topograph.

2. Specify a set-point voltage and current, $V_{s}$ and $I_{s}$, which will be used at all pixels to establish a tunnel junction. With the feedback loop turned on and a bias voltage $V_{s}$ applied, the tip is moved to the first point in the measurement array and allowed to attain the set-point current $I_{s}$ by varying $z$.

3. The feedback loop is then turned off and the value of $z$ recorded as $T\left(\vec{r}_{1}\right)$.

4. An AC voltage modulation is then added to the DC bias. The DC bias $V_{0}$ is sequentially moved through a series of values $V_{1}, V_{2}, \ldots, V_{i}, \ldots, V_{M}$, pausing at each to average and record the values of current and differential conductance as $I\left(\vec{r}_{1}, E_{i}=e V_{i}\right)$ and $g\left(\vec{r}_{1}, E_{i}=e V_{i}\right)$. These are the spectra that make up the first pixel of the spectroscopic maps $I(\vec{r}, E)$ and $g(\vec{r}, E)$.

5. Ramp voltage back to $V_{S}$, turn on feedback loop and move to next pixel in array. Repeat steps 3-5 until a measurement has been made at every pixel

The results of this procedure are spectroscopic maps $I(\vec{r}, E)$ and $g(\vec{r}, E)$ measured for $\vec{r}=\vec{r}_{1}, \vec{r}_{2} \ldots \vec{r}_{N}$ and $E=e V_{1}, V_{2}, \ldots, V_{i}, \ldots, V_{M}$, as well as the simultaneous topograph $T(\vec{r})$. The SI-STM data collection modality and resultant data are depicted in figure 2.5 . 

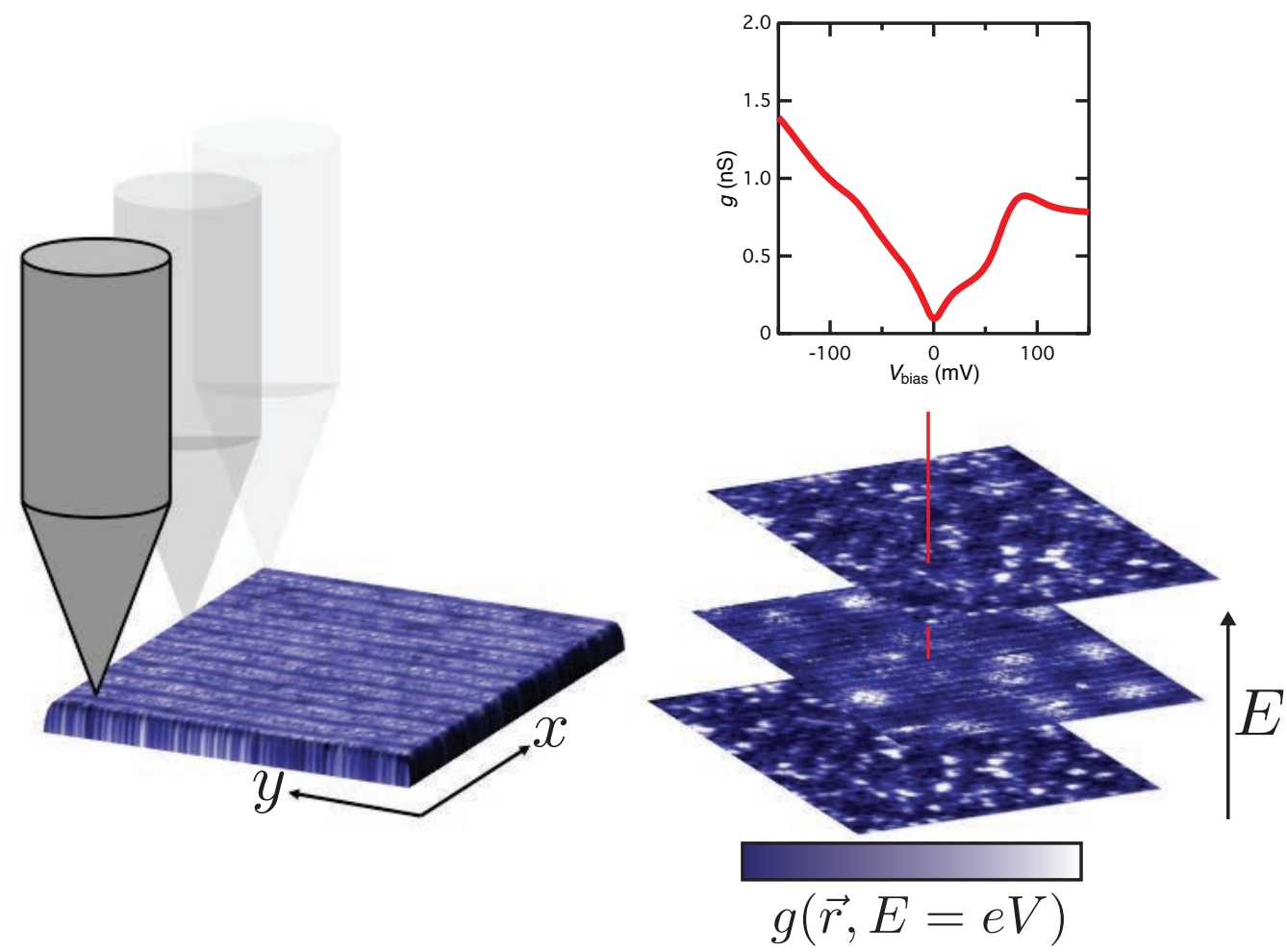

FIgURE 2.5: Schematic illustration of SI-STM. The STM tip sequentially establishes a junction at an array of points on the surface of the sample and records current and conductance spectrum at each. This results in spectroscopic maps $I(\vec{r}, E)$ and $g(\vec{r}, E)$ as well a simultaneous topograph $T(\vec{r}) . g(\vec{r}, E)$ is shown on the left hand side in three dimensions $(x, y, E)$. The colour scale in each slice corresponds to $g(\vec{r}, E)$ at a fixed energy. The red line parallel to the energy axis shows the path through this 3D space which results in the conductance spectrum (also shown in red) at a fixed point on the sample.

\subsubsection{The Setup Effect}

SI-STM provides energy resolved electronic structure images that can be used to identify the presence of LDOS modulations. However the protocol described above, by which a tunnelling junction is established in SI-STM, can imprint conductance modulations from one set of energies onto another. This systematic error, which results in a misidentification of the energy of states undergoing spatial modulations, is called the setup effect. Therefore, determining the physically real modulations and especially their correct energies can present a grave challenge. Ignoring the setup effect, as is often the case, leads to incorrect characterisation of the electronic structure of materials.

The basic observable in STM experiments is the tunnelling current, $I(\vec{r}, z, E=e V)$, which depends on the bias between the tip and the sample, $V$, the tip sample separation, $z$, and the position on the sample surface, $r=\overrightarrow{(x}, y)$, according to 


$$
I(\vec{r}, z, V)=f(\vec{r}, z) \int_{0}^{e V} n(\vec{r}, \epsilon) d \epsilon
$$

The function $f(\vec{r}, z)$ captures spatial variations due to surface corrugation, work function, matrix elements and proximity $z$ of the tip to the surface. The integral of the LDOS, $\int_{0}^{e V} n(\vec{r}, \epsilon) d \epsilon$, includes spatial variation of the electronic structure.

SI-STM entails establishing a stable tunnel junction at every $\vec{r}$ by using the same setpoint current and bias voltage $I_{S}$ and $V_{S}$. These set-point values then constrain the pre-factor of the integral in equation 2.33 via

$$
\begin{gathered}
I\left(\vec{r}, z, V_{S}\right)=I_{S}=f(\vec{r}, z) \int_{0}^{e V_{S}} n(\vec{r}, \epsilon) d \epsilon \\
\Rightarrow f(\vec{r}, z)=\frac{I_{S}}{\int_{0}^{e V_{S}} n(\vec{r}, \epsilon) d \epsilon}
\end{gathered}
$$

and thus the expressions for $I(\vec{r}, E=e V)$ and $g(\vec{r}, E=e V)$ are given by

$$
\begin{gathered}
I(\vec{r}, E=e V)=I_{S} \frac{\int_{0}^{e V} n(\vec{r}, \epsilon) d \epsilon}{\int_{0}^{e V_{S}} n(\vec{r}, \epsilon) d \epsilon} \\
g(\vec{r}, E=e V)=I_{S} \frac{n(\vec{r}, e V)}{\int_{0}^{e V_{S}} n(\vec{r}, \epsilon) d \epsilon} .
\end{gathered}
$$

The term in the denominator is responsible for the setup effect since it imprints the integral of the LDOS over the range of energies 0 to $e V_{s}$ onto every energy in $I(\vec{r}, E=e V)$ or $g(\vec{r}, E=e V)$.

Figure 2.6 demonstrates how the choice of setup bias to establish the tunnelling junction strongly influences the acquired data. While both the left and right panels are spatial images of $g(\vec{r}, E=e V)$ taken at $V=50 \mathrm{mV}$ in the same field of view, the left was measured with $V_{S}=150 \mathrm{mV}$ setup bias while the right with $V_{S}=50 \mathrm{mV}$. It is clear that the spatial patterns of the same set of states in the material are imaged differently based on the setup bias parameter.

In order to faithfully resolve which modulations in $g(\vec{r}, E)$ arise from which energies in the LDOS, one must find a setup bias, $V_{S}$, for which $\int_{0}^{e V_{S}} n(\vec{r}, \epsilon) d \epsilon$ does not exhibit any modulations at the wave-vector of interest. In general, this is difficult because if $n(\vec{r}, E)$, for $E<e V_{S}$, has modulations at wave-vector $\vec{Q}$ then it is likely that $\int_{0}^{e V_{S}} n(\vec{r}, \epsilon) d \epsilon$ also will, unless there is an evolution of the modulation phase with energy. 

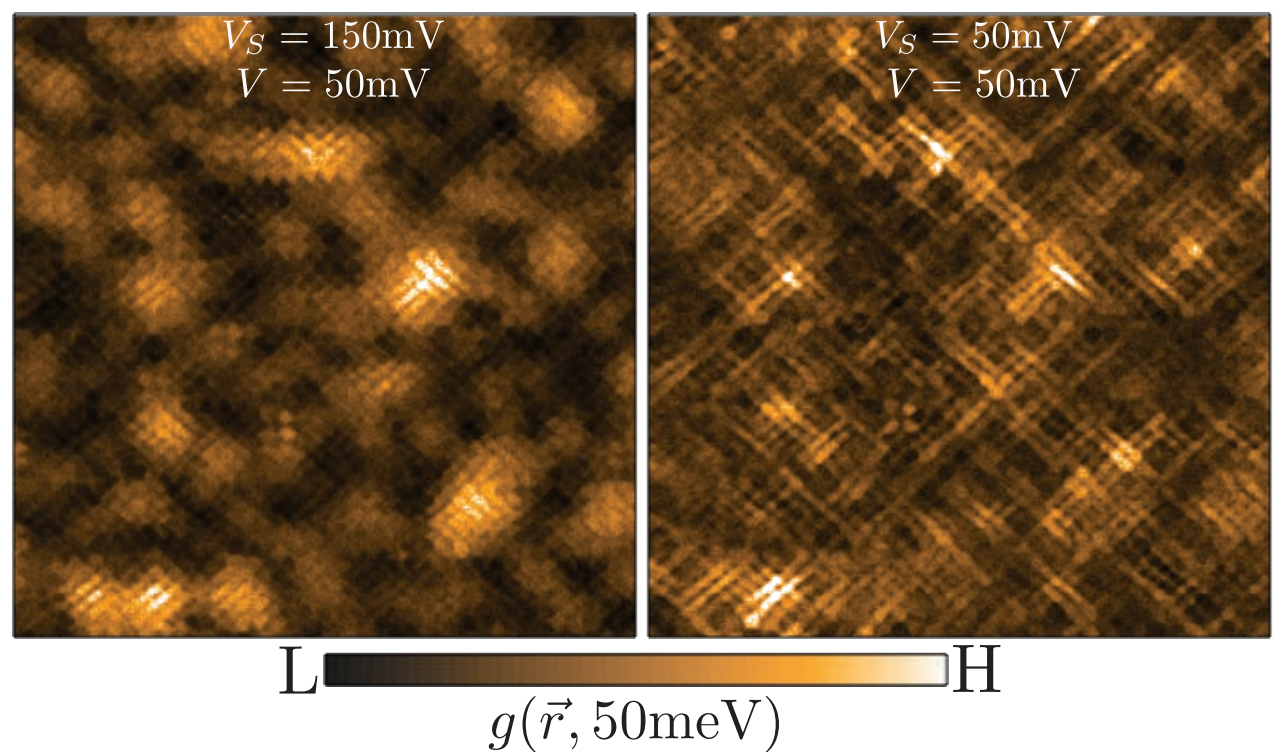

Figure 2.6: The two panels show the spatial differential conductance of an underdoped $\mathrm{Bi}_{2} \mathrm{Sr}_{2} \mathrm{CaCu}_{2} \mathrm{O}_{8+\delta}$ sample at $E=50 \mathrm{meV}$ acquired in the same field of view. The left panel was acquired with a setpoint bias of $V_{S}=150 \mathrm{mV}$ while the right with one of $V_{S}=150 \mathrm{mV}$.

In cases where no suitable set-point can be found, we can use ratios of $g(\vec{r}, E=e V)$ at different voltages, typically of equal magnitude but opposite polarity. Such combinations have the effect of cancelling the setup effect described here and will be discussed in the following section.

\subsubsection{Spectroscopic Functions $g(\vec{r}, E), I(\vec{r}, E), Z(\vec{r}, E)$, and $R(\vec{r}, E)$}

I will now consider how, by defining new spectroscopic functions, one can remove the setup effect described in section 2.4.1. Equations 2.36 and 2.37 show us that at a given location, $\vec{r}$, and setup bias, $V_{S}$, the conductance and current values at all bias voltages contain the same constant factor $I_{S} / \int_{0}^{e V_{S}} n(\epsilon, \vec{r}) d \epsilon$. Thus, by defining a function that is a ratio of two conductances or currents at the same location, one obtains a function that is independent of $V_{S}$ and hence setup effect free. In doing so, however, one must combine conductance or current values from different bias voltages and convolve LDOS modulations from different energies. 
In this thesis the two setup free functions analysed are

$$
Z(\vec{r},|E|)=\frac{g(\vec{r},+|E|)}{g(\vec{r},-|E|)}=\frac{n(\vec{r},+|E|)}{n(\vec{r},-|E|}
$$

and

$$
R(\vec{r},|E|)=\frac{I(\vec{r},+|E|)}{I(\vec{r},-|E|)}=\frac{\int_{0}^{|E|} n(\vec{r}, \epsilon) d \epsilon}{\int_{0}^{|E|} n(\vec{r},-\epsilon) d \epsilon} .
$$

These functions remove the setup effect by sacrificing the distinction between filled and empty states (negative and positive bias voltages respectively). $Z(\vec{r},|E|)$ is the ratio of tunnelling probabilities for states at opposite polarity bias and similarly $R(\vec{r},|E|)$ is the ratio of integrated tunnelling probabilities. As such they are often called tunnelling asymmetries.

One effect of these functions is that they naturally enhance any modulations present in the tunnelling conductance that have a $\pi$ phase shift between states above and below the Fermi energy. As we will see in chapter 4, this is the case for the predominantly $d$-symmetry form factor CDW present in $\mathrm{Bi}_{2} \mathrm{Sr}_{2} \mathrm{CaCu}_{2} \mathrm{O}_{8+\delta}$ and $\mathrm{Na}_{x} \mathrm{Ca}_{2-x} \mathrm{CuO}_{2} \mathrm{Cl}_{2}$. The use of $Z(\vec{r},|E|)$ and $R(\vec{r},|E|)$ naturally enhances the signals arising from these phenomena.

While $Z(\vec{r},|E|)$ measures the tunnelling asymmetry between states at energies $e V$ above and below the Fermi energy, $R(\vec{r},|E|)$ measures the tunnelling asymmetry of all states up to an energy $\mathrm{eV}$ above and below the Fermi energy. $R(\vec{r},|E|)$ then naturally gains a greater weight from modulations that are non-dispersive in energy, such as those from a charge density wave.

The final benefit of $Z(\vec{r},|E|)$ and $R(\vec{r},|E|)$ is that they correct for digitisation errors in the junction setup process. This is not a fundamental problem (as with the setup effect) but a purely technical malady. The high voltages applied to the piezo scanner tube are produced by a 16-bit digital to analogue converter (DAC) whose output is passed through a high voltage amplifier. The bin size of the DAC then determines a precision with which the set-point current can be attained by z-axis motion of the STM tip. This means that all pixels in an STM are taken at very slightly different values of $I_{S}$. Under the low mechanical and electrical noise conditions used for data acquisition in this thesis, this can be the dominant source of noise in the Fourier transform of $g(\vec{r}, V)$. Luckily, 
equations $2.36,2.37,2.38$ and 2.39 imply that this effect is cancelled exactly in $Z(\vec{r},|E|)$ and $R(\vec{r},|E|)$.

\subsection{Experimental Apparatus}

I will now describe briefly the specific experimental implementation of cryogenic SI-STM used to collect the data presented in this thesis.

\subsubsection{STM Head}

The STMs used in these studies all follow a construction similar to that shown in figure 2.7. Their design and construction is detailed in reference [78].

The STM tip is held atop a split electrode piezoelectric tube. By applying voltages to the electrodes, the $\mathrm{x}, \mathrm{y}$ and $\mathrm{z}$ position of the tip can be controlled with sub-Angström precision over a total range of $\sim 1 \mu \mathrm{m}$. This tube is held inside a sapphire prism. This can be actuated up and down by the slip-stick motion of shear piezo stacks, giving coarse positioning in the z-direction. This assembly is housed inside a rigid Macor body, designed to give its vibrational modes as high a frequency as possible.

Sample are inserted into the top of the STM head on brass sample holders which mate with the head assembly and are held tightly in place by a BeCu spring plate. This plate also applies the bias voltage to the sample, with the tip being held at ground.

Unless otherwise stated all measurements reported in this thesis were made using a chemically etched tungsten STM tip.

\subsubsection{Cryogenics}

The data presented in this thesis were taken at temperatures between $45 \mathrm{~K}$ and $45 \mathrm{mK}$. All instruments were mounted in vacuum cans immersed in liquid ${ }^{4}$ He resulting in a cryogenic ultra-high vacuum where the STM head is housed. The measurements presented in chapters 5 and 7 utilise a dilution refrigerator.

All samples were inserted from room temperature into the vacuum using an insertion rod and cleaved at $\sim 4 \mathrm{~K}$. From here they were then inserted directly into the STM head. 

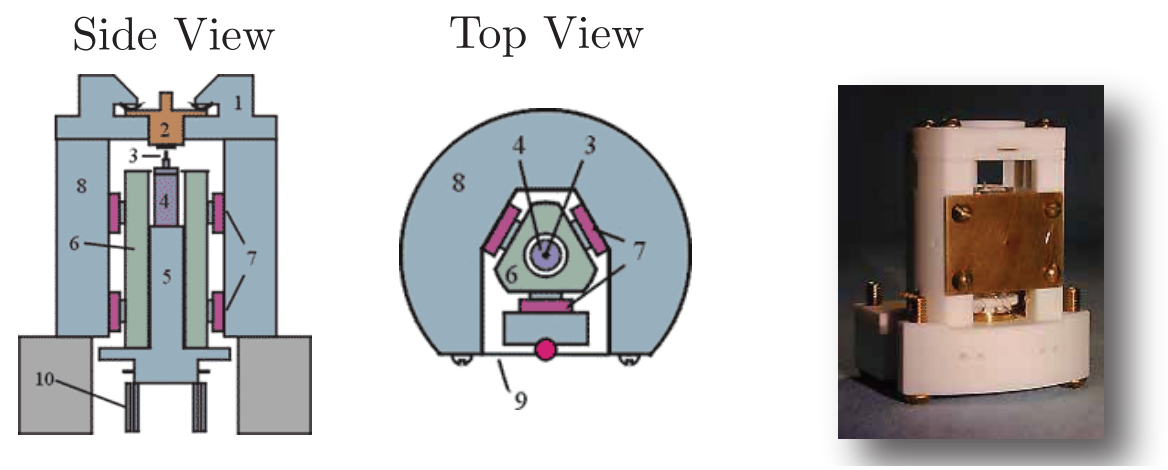

FiguRe 2.7: Schematic drawing and photograph of STM head. Parts labelled 1-10 are: 1) Sample Receptacle 2) Sample Holder 3) Tip 4) Piezo-tube Scanner 5) Scanner Holder 6) Sapphire Prism 7) Shear Piezo Stacks 8) Macor Body 9) BeCu Spring Plate

10) Capacitive Position Sensor.

\subsubsection{Vibration and RF Isolation}

Mechanical vibrations introduce noise into STM measurements primarily by varying the tip-sample distance. To maximise the signal to noise ratio of our measurement we utilise passive vibration isolation. This method relies on successive stages having very different resonant frequencies. A concrete block $\left(\sim 10\right.$ tonnes, $\left.f_{\text {res }} \sim 1 \mathrm{~Hz}\right)$ is separated from the foundations of the building and supported on air springs. The cryostat is then suspended from a vibrational isolation table $\left(\sim 1\right.$ tonne, $\left.f_{\text {res }} \sim 10 \mathrm{~Hz}\right)$, again using air springs.

Acoustic isolation is achieved by constructing a room around the cryostat on the floating block, incorporating sound absorbing material. This is then housed inside another room whose walls are filled with sand and the interior lined with sound absorbing material. This is shown in figure 2.8 .

For the scanned Josephson experiments reported in chapters 5 and 7 the floating experimental room is RF shielded.

\subsubsection{Instrumentation}

The instrumentation used in our SI-STM measurements is shown in figure 2.9. Tipsample current measurements are made with a low-noise current to voltage pre-amplifier situated inside the floating experimental room. Differential conductance measurements are made using a lock-in amplifier. The DC bias voltage, feedback loop and high voltage signal to control the piezoelectrics are provided by a Topometrix SPM controller, interfaced to a computer running custom designed control software [80]. 


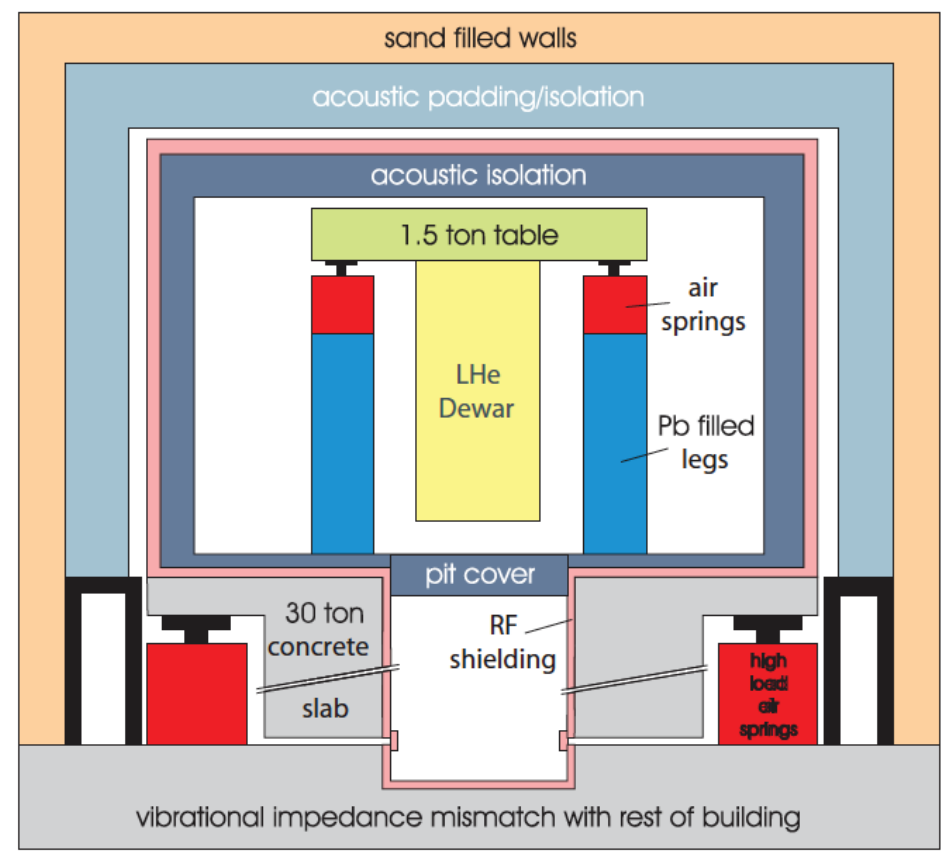

Figure 2.8: Passive vibration isolation utilised in SI-STM experiments. Reproduced from reference [79]

\subsection{Cuprate Materials}

In this thesis, SI-STM and SJTM are applied to cuprate superconductors to visualise charge density wave (CDW) modulations as well as those in the Cooper-pair condensate. Here I will briefly describe the cuprate samples studied.

The common feature of all cuprate superconductors are the $\mathrm{CuO}_{2}$ planes. The variety in this family of materials arises from how these planes are incorporated into a crystal. In this thesis measurements of two cuprate materials, $\mathrm{Bi}_{2} \mathrm{Sr}_{2} \mathrm{CaCu}_{2} \mathrm{O}_{8+\delta}$ and $\mathrm{Na}_{x} \mathrm{Ca}_{2-x} \mathrm{CuO}_{2} \mathrm{Cl}_{2}$, are presented. These materials were chosen for their compatibility with SI-STM because they both cleave easily and reproducibly to yield atomically flat surfaces that are not reconstructed. Below I will briefly describe the crystallography and properties of these two cuprate superconductors, as well as the fabrication of the samples measured in our studies.

\subsection{1 $\quad \mathrm{Bi}_{2} \mathrm{Sr}_{2} \mathrm{CaCu}_{2} \mathrm{O}_{8+\delta}$}

In $\mathrm{Bi}_{2} \mathrm{Sr}_{2} \mathrm{CaCu}_{2} \mathrm{O}_{8+\delta}$ the $\mathrm{CuO}_{2}$ planes are sandwiched between $\mathrm{BiO}, \mathrm{SrO}$ and $\mathrm{Ca}$ 


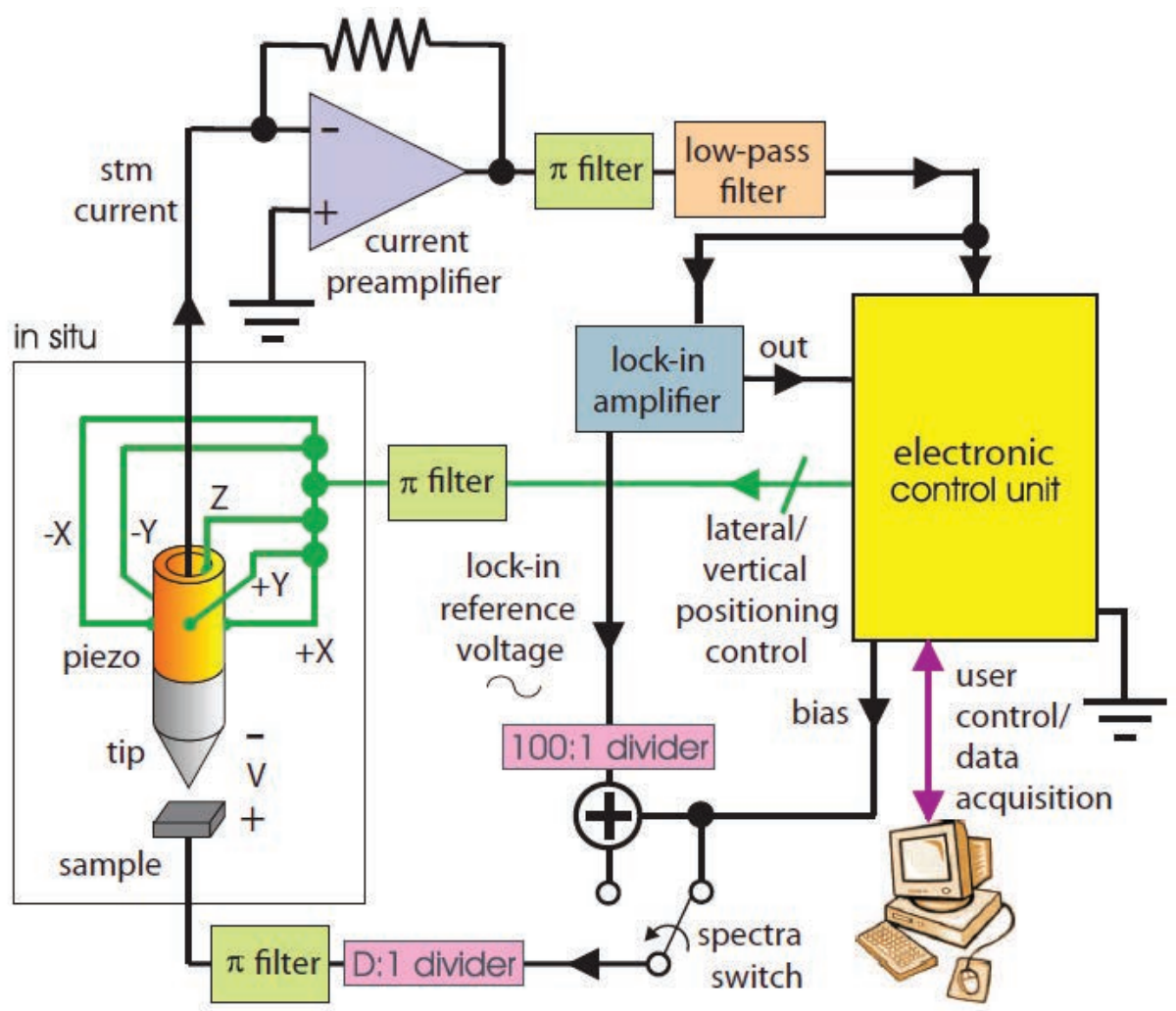

FigURE 2.9: A bias applied to the sample is sourced from the electronic control unit (ECU), a Topometrix SPM controller. The resulting tunnelling current is measured using a low noise current-voltage pre-amplifier situated at the cryostat head in the floating experimental room. The position of the tip is controlled by a piezoelectric scanner tube. The control voltages for this are supplied by the ECU. In topographic mode the $z$ piezo-tube voltage is controlled by a PID feedback loop in the ECU to keep the tunnelling current constant. In spectroscopic mode, this feedback loop is turned of and a constant $z$ piezo voltage used. An AC bias modulation from a lock-in amplifier is added to the sample bias. This lock-in amplifier measures the differential conductance through the resulting AC modulation in the tunnelling current. Reproduced from reference [81].

layers, as shown in figure 2.10. There are two $\mathrm{CuO}_{2}$ layers per $\mathrm{BiO}$ layer, leading to the description of $\mathrm{Bi}_{2} \mathrm{Sr}_{2} \mathrm{CaCu}_{3} \mathrm{O}_{10+\delta}$ as a bi-layer compound, with $\mathrm{Bi}_{2} \mathrm{Sr}_{2} \mathrm{CuO}_{6+\delta}$ and $\mathrm{Bi}_{2} \mathrm{Sr}_{2} \mathrm{Ca}_{2} \mathrm{Cu}_{2} \mathrm{O}_{8+\delta}$ being its single and triple layer relatives respectively. $\mathrm{Bi}_{2} \mathrm{Sr}_{2} \mathrm{CaCu}_{2} \mathrm{O}_{8+\delta}$ has the $\mathrm{I}_{4} / m m m$ space group with $4 \mathrm{CuO}_{2}$ planes per unit cell, with an offset of $(1 / 2,0,0)$ between the two bilayers. The mirror plane between adjacent $\mathrm{BiO}$ layers leads to $\mathrm{BiO}$ being the natural cleave plane of the material. Note that bismuth atoms always lie directly above copper atoms, allowing one to determine the copper positions from the surface topography. The nearest neighbour distance between copper atoms forming approximately square lattice planes is $3.82 \AA$.

The $\mathrm{CuO}_{2}$ plaquettes are not exactly square in $\mathrm{Bi}_{2} \mathrm{Sr}_{2} \mathrm{CaCu}_{2} \mathrm{O}_{8+\delta}$ due to the presence 
of a bulk incommensurate structural super-modulation [82, 83]. This buckling of the whole crystal, at $45^{\circ}$ to the $\mathrm{Cu}-\mathrm{O}$ directions (along the b-axis in figure 2.10) results from a lattice mismatch between the $\mathrm{BiO}$ and other layers of the crystal. Importantly, this modulation leaves intact a mirror symmetry between the two $\mathrm{Cu}-\mathrm{O}$ directions in the $\mathrm{CuO}_{2}$ plane. Thus, any breaking of this symmetry is spontaneous.

The $\mathrm{Bi}_{2} \mathrm{Sr}_{2} \mathrm{CaCu}_{2} \mathrm{O}_{8+\delta}$ samples used for the measurements presented here were grown by K. Fujita in the group of S. Uchida at the University of Tokyo. For hole dopings $p \leq 0.08$ $\mathrm{Bi}_{2.2} \mathrm{Sr}_{1.8} \mathrm{Ca}_{0.8} \mathrm{Dy}_{0.2} \mathrm{Cu}_{2} \mathrm{O}_{8+\delta}$ samples were used whereas for $p>0.08$ the composition $\mathrm{Bi}_{2.1} \mathrm{Sr}_{1.9} \mathrm{CaCu}_{2} \mathrm{O}_{8+\delta}$ was employed.

High-quality single crystals were grown using the travelling solvent floating zone method. The samples were synthesised from dried powders of $\mathrm{Bi}_{2} \mathrm{O}_{3}, \mathrm{SrCO}_{3}, \mathrm{CaCO}_{3}$ and $\mathrm{CuO}$. The crystal growth was carried out in air and at growth speeds of $0.15-0.2 \mathrm{~mm} / \mathrm{h}$ for all samples. Annealing was used to vary the hole doping of each sample. Oxidation annealing is performed in air or under oxygen gas flow, and deoxidation annealing is done in vacuum or under nitrogen gas flow. Inductively coupled plasma spectroscopy was used for the composition analysis and a vibrating sample magnetometer was used for measurement of $T_{c}$. Each sample was inserted into the cryogenic ultra-high vacuum of the SI-STM system and cleaved at $T \approx 4 \mathrm{~K}$ to reveal an atomically flat $\mathrm{BiO}$ surface.

\subsection{2 $\mathrm{Na}_{x} \mathrm{Ca}_{2-x} \mathrm{CuO}_{2} \mathrm{Cl}_{2}$}

The other material for which measurements are reported in this thesis is $\mathrm{Na}_{x} \mathrm{Ca}_{2-x} \mathrm{CuO}_{2} \mathrm{Cl}_{2}$. In this material the $\mathrm{CuO}_{2}$ planes are incorporated between $\mathrm{CaCl}$ layers, as shown in figure 2.11. The material is tetragonal, with the $\mathrm{I}_{4} / \mathrm{mmm}$ space group and does not have any incommensurate structural super-modulation. Hole doping is achieved by substituting $\mathrm{Ca}^{2+}$ ions for $\mathrm{Na}^{+}$ions, introducing holes into the $\mathrm{CuO}_{2}$ planes. In this thesis I present data from $\mathrm{Na}_{x} \mathrm{Ca}_{2-x} \mathrm{CuO}_{2} \mathrm{Cl}_{2}$ samples with $p=12 \%$.

The $\mathrm{Na}_{x} \mathrm{Ca}_{2-x} \mathrm{CuO}_{2} \mathrm{Cl}_{2}$ samples were grown by $\mathrm{Y}$. Kohsaka at the University of Tokyo [85]. A flux method was used for growth, with high pressures used to increase the solubility of Na. The samples were synthesised from powders of $\mathrm{Ca}_{2} \mathrm{CuO}_{2} \mathrm{Cl}_{2}, \mathrm{NaClO}_{4}$ and $\mathrm{NaCl}$ in a high pressure anvil cell at pressures of 2-5.5 GPa. 


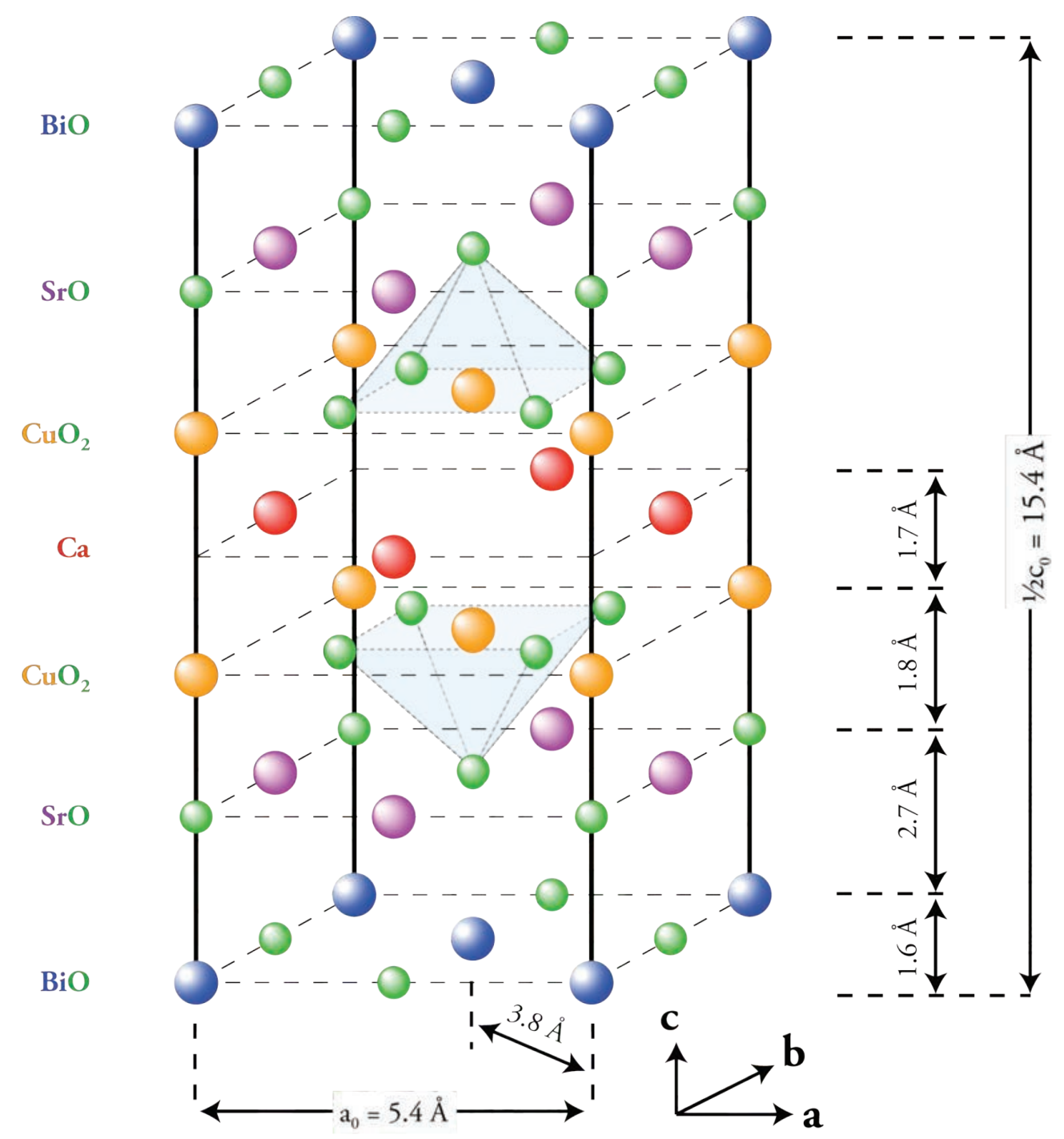

Figure 2.10: Cartoon unit cell of the bi-layer cuprate $\mathrm{Bi}_{2} \mathrm{Sr}_{2} \mathrm{CaCu}_{2} \mathrm{O}_{8+\delta} \cdot \mathrm{CuO}_{2}$ planes are incorporated between $\mathrm{Ca}, \mathrm{SrO}$ and $\mathrm{BiO}$ layers. Only half of the unit cell is shown along the c-axis. The second half of the unit cell is a repeat of that shown but offset by $(1 / 2,0,0)$. The natural cleavage plane is the $\mathrm{BiO}$ layer. $\mathrm{Bi}$ atoms are directly above the $\mathrm{Cu}$ atoms in the crystal structure allowing the $\mathrm{Cu}$ positions to be identified from surface topography. Reproduced from reference [84].

\subsection{Quasi-Particle Interference in Cuprates}

Quasi-particle interference (QPI) is a technique used to extract the energy-momentum dispersion of quasi-particles in a material using SI-STM $[27,28,86]$. It uses the characteristic wave-lengths present in the density of states modulations formed by quasiparticles scattering from impurities, often called Friedel oscillations. With a suitable model, one can use the bias voltage dependence of these characteristic wave-lengths to determine the quasi-particle dispersion. 


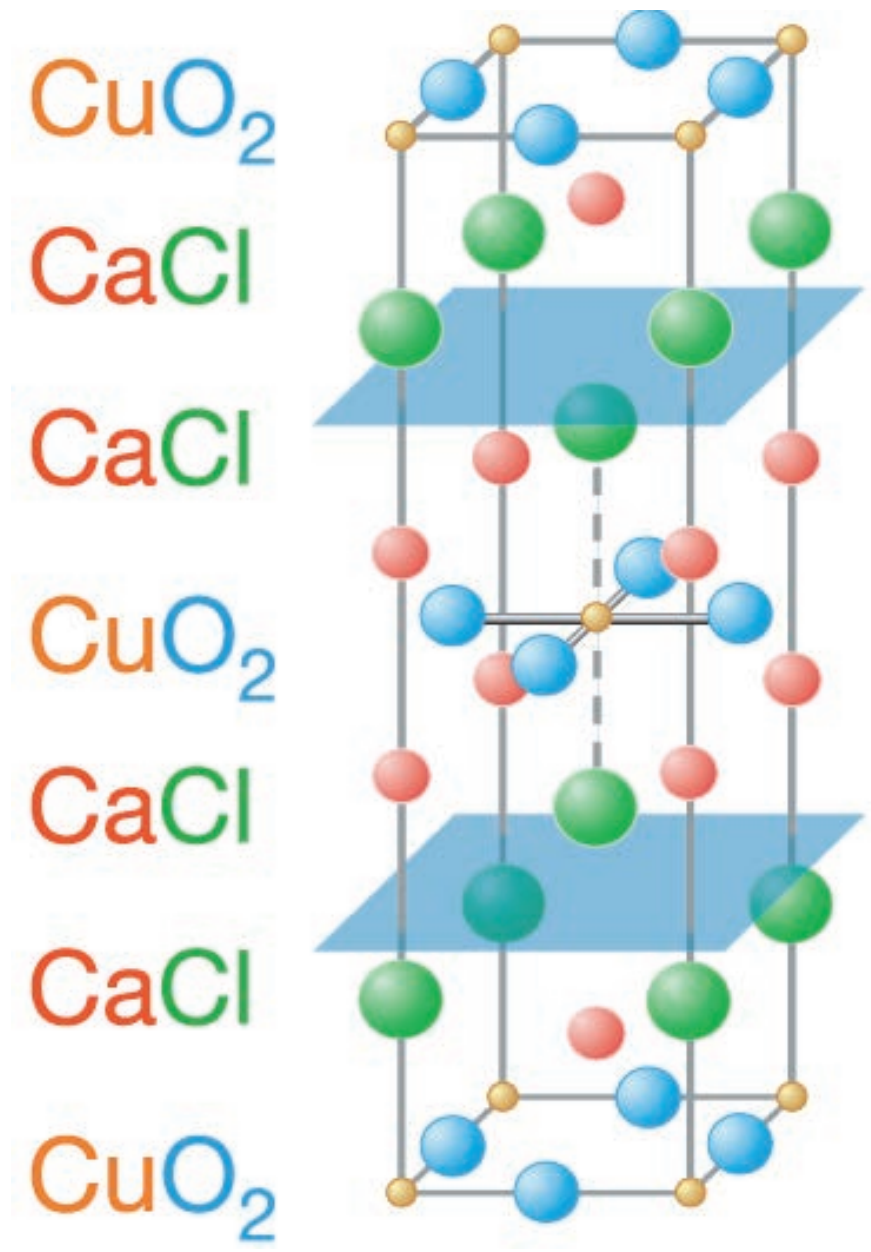

Figure 2.11: Cartoon unit cell of $\mathrm{Na}_{x} \mathrm{Ca}_{2-x} \mathrm{CuO}_{2} \mathrm{Cl}_{2} \cdot \mathrm{CuO}_{2}$ planes are incorporated between $\mathrm{CaCl}$ layers. Hole doping is achieved by substituting $\mathrm{Ca}^{2+}$ ions for $\mathrm{Na}^{+}$ions, introducing holes into the $\mathrm{CuO}_{2}$ planes. Reproduced from reference [46].

This technique is applicable to both normal Landau quasi-particles as well as Bogoliubov quasi-particles in a superconductor. In chapter $4 \mathrm{I}$ will use Bogoliubov quasi-particle interference in $\mathrm{Bi}_{2} \mathrm{Sr}_{2} \mathrm{CaCu}_{2} \mathrm{O}_{8+\delta}$ to determine geometric details of the Fermi surface. In the following I will outline how this technique works and its specific application to cuprates.

\subsubsection{QPI - A Qualitative Understanding}

For a clean metal where the quasi-particle states are described by Bloch functions, $\Psi_{\vec{k}}=$ $e^{i \vec{k} \cdot \vec{r}} u_{\vec{k}}(\vec{r})$, the spatial dependence of the LDOS, $n(\vec{r}, E=e V s)=\sum_{\vec{k}}\left|\Psi_{\vec{k}}\right|^{2} \delta\left(E-\epsilon_{\vec{k}}\right)$, only contains modulations with the periodicity of the atomic lattice, encoded in $u_{\vec{k}}$. 
The introduction of impurities into the lattice leads to elastic scattering between Bloch states, which are no longer the true eigenstates. The new eigenstates will be linear superpositions of Bloch states that lie on the same constant energy contour in $\vec{k}$-space. This mixing of $\vec{k}$-states leads to oscillations in $n(\vec{r}, E)$ with wave-vectors $\{\vec{q}\}$ linking points on the contour of constant energy $E$. This can be illustrated as follows:

$$
\begin{aligned}
|\Psi(\vec{r})|^{2} & =\left|a_{1} u_{\overrightarrow{k_{1}}}(\vec{r}) e^{i \overrightarrow{k_{1} \cdot \vec{r}}}+a_{2} u_{\overrightarrow{k_{2}}}(\vec{r}) e^{i \overrightarrow{k_{2}} \cdot \vec{r}}\right|^{2} \\
& =\left|a_{1} u_{\overrightarrow{k_{1}}}(\vec{r})\right|^{2}+\left|a_{2} u_{\overrightarrow{k_{2}}}(\vec{r})\right|^{2}+\left(a_{1} u_{\overrightarrow{k_{1}}}(\vec{r}) a_{2}^{*} u_{\overrightarrow{k_{2}}}^{*}(\vec{r}) e^{i \vec{q} \cdot \vec{r}}+c . c\right)
\end{aligned}
$$

where $\vec{q}=\overrightarrow{k_{1}}-\overrightarrow{k_{2}}$. This will be observed as modulations of the local density of states, $n(\vec{r}, E)$ with wavelength $\lambda=\frac{2 \pi}{|\vec{q}|}$.

As discussed above in section 2.4, SI-STM yields spectroscopic maps $I(\vec{r}, E)$ and $g(\vec{r}, E)$. Further, $g(\vec{r}, E)$ is proportional to the local density of states $n(\vec{r}, E)$. For a fixed energy, $g(\vec{r}, E)$ is a two-dimensional image and will contain quasi-particle interference modulations from scattering between the contours of constant energy $E$. By taking a two-dimensional Fourier transform to yield $\tilde{g}(\vec{q}, E)$ we can extract the LDOS modulation wave-vectors and hence infer geometric information about the constant energy contours (CCEs) in the quasi-particle dispersion. By tracking the wave-vectors present in these Fourier transforms systematically with energy we can potentially extract the quasi-particle dispersion in a material.

To illustrate this consider a quasi-2D material with a cylindrical Fermi surface with Fermi wavevector $\left|\overrightarrow{k_{F}}\right|$ in the presence of a point impurity (whose scattering form factor will be momentum independent). The power spectrum of a conductance map taken at zero bias will show a strong cusp at a circle of radius $|\vec{q}|=2\left|\overrightarrow{k_{F}}\right|$ because this is where the joint density of states for scattering is largest.

In this example it is easy to work backwards from the power spectrum to deduce the Fermi-surface and by varying the bias we can determine the dispersion relation. However, in cases where the scattering potential is not isotropic or the constant energy contours are complicated this process becomes non-trivial and is one of the major challenges of analysing this type of experiment. Luckily in cuprates, we have a well established model of Bogoliubov quasi-particle interference called the "octet model", which I will now introduce. 


\subsubsection{The Octet Model}

It is well established that cuprate superconductors exhibit a $d$-wave superconducting gap of the form,

$$
\Delta_{\vec{k}}=\frac{\Delta_{0}}{2}\left(\cos \left(k_{x} a_{0}\right)-\cos \left(k_{y} a_{0}\right)\right),
$$

with four nodes along the zone diagonals. This leads to a Bogoliubov quasi-particle dispersion

$$
E_{\vec{k}}=\sqrt{\epsilon_{\vec{k}}^{2}+\Delta_{\vec{k}}^{2}}
$$

where $\epsilon_{\vec{k}}$ is the dispersion of the underlying normal band. In the cuprates $\partial_{k_{\perp}} \Delta_{\vec{k}} \ll \partial_{k_{\|}} \epsilon_{\vec{k}}$ $\left(\partial_{k_{\|}} \epsilon_{\vec{k}} / \partial_{k_{\perp}} \Delta_{\vec{k}} \approx 20\right.$ at the gap nodes [87]), where $k_{\perp}$ and $k_{\|}$are perpendicular and parallel to the Fermi wave-vector respectively. This results in banana-like constant energy contours (CECs) in the Bogoliubon dispersion as shown in the figure 2.12.

(a)

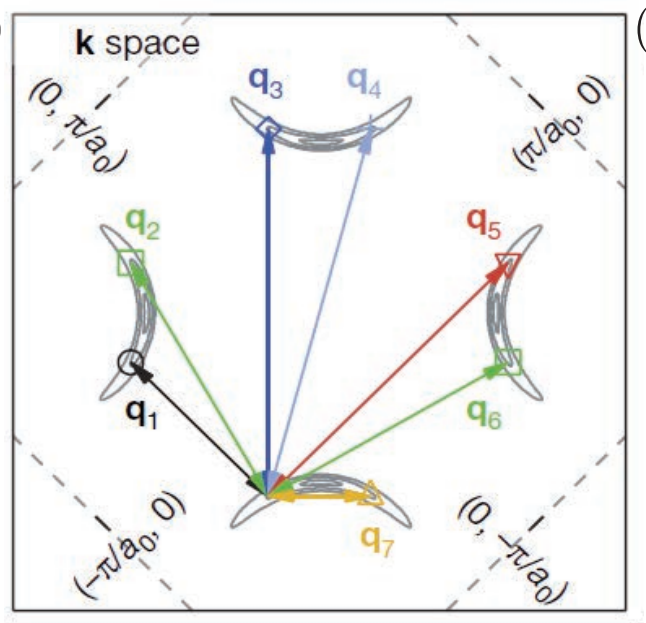

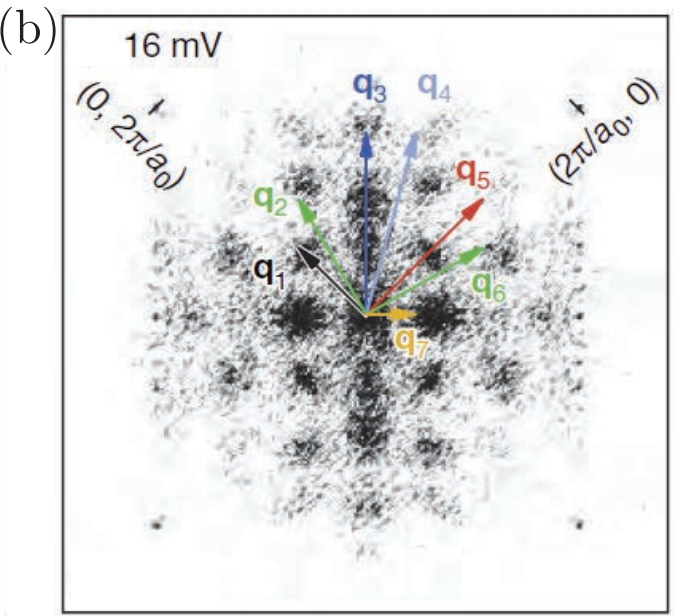

Figure 2.12: (a) Banana shaped constant energy contours (CECs) of the $d$-wave Bogoliubov quasi-particle dispersion in $\vec{k}$-space. In the octet model, quasi-particle interference modulations in the LDOS primarily arise from scattering between the eight tips of these bananas. This results in 7 scattering vectors $\vec{q}_{1}, \ldots, \vec{q}_{7}$. (b) Measured fourier transform $Z(\vec{q},|E|)$ from underdoped $\mathrm{Bi}_{2} \mathrm{Sr}_{2} \mathrm{CaCu}_{2} \mathrm{O}_{8+\delta}$ shown LDOS modulations at the wave-vectors $\vec{q}_{1}, \ldots, \vec{q}_{7}$. Reproduced from reference [27].

As discussed in the previous section, the strongest modulations are usually seen at wavevectors for which the joint density of states for scattering between points on the CECs are maximal. One way this can arise is through nesting, where large sections of a Fermi surface are parallel to each other in $\vec{k}$-space. 
In cuprates the situation is different. The points with the largest density of states

$$
n(\vec{k}, E) \propto \frac{1}{\left|\nabla_{\vec{k}}(E)\right|}
$$

are those at the eight tips of the banana like CECs. In the absence of nesting, the largest joint density of states for scattering will also be between these eight points [70, 87]. Thus QPI LDOS modulations will arise from the set of $8 \times 7=56$ pairings that connect these.

These 56 pairings are not all distinct because the same scattering vector connects multiple points. Taking this into account there are 16 independent $\vec{q}$ vector measurements that we can make at each bias voltage. But again, some of these are related by the crystal symmetry, allowing us to make multiple independent measurements of the 7 scattering vectors,

$$
\begin{aligned}
& \vec{q}_{1}(E)=\left(2 k_{x}, 0\right) \\
& \vec{q}_{2}(E)=\left(k_{x}+k_{y}, k_{y}-k_{x}\right) \\
& \vec{q}_{3}(E)=\left(k_{x}+k_{y}, k_{x}+k_{y}\right) \\
& \vec{q}_{4}(E)=\left(2 k_{x}, 2 k_{y}\right) \\
& \vec{q}_{5}(E)=\left(0,2 k_{y}\right) \\
& \vec{q}_{6}(E)=\left(k_{x}-k_{y}, k_{x}+k_{y}\right) \\
& \vec{q}_{7}(E)=\left(k_{x}-k_{y}, k_{y}-k_{x}\right),
\end{aligned}
$$

where $\vec{k}=\left(k_{x}, k_{y}\right)$ corresponds to the momentum space coordinates of the banana tips at a given energy.

Ultimately, we wish to determine an energy momentum-dispersion relation $\vec{k}(E)$. To do this we can invert equations 2.45-2.51 to yield,

$$
\begin{aligned}
& 2 k_{x}(E)=\left|\vec{q}_{1}\right|, q_{2 x}-q_{2 y}, q_{6 y}-q_{6 x},\left|\vec{q}_{3}-\vec{q}_{7}\right| / \sqrt{2},\left|\vec{q}_{5}\right|-\sqrt{2}\left|\vec{q}_{7}\right|, q_{4 x} \\
& 2 k_{y}(E)=\left|\vec{q}_{5}\right|, q_{2 x}+q_{2 y}, q_{6 y}+q_{6 x},\left|\vec{q}_{3}+\vec{q}_{7}\right| / \sqrt{2}, \sqrt{2}\left|\vec{q}_{7}\right|-\left|\vec{q}_{1}\right|, q_{4 y} .
\end{aligned}
$$

These expressions show that this octet model is geometrically over-constrained. At each energy, $E$, we are able to make 6 independent estimates of $\vec{k}(E)$. Although I have given 
some justification for this octet model above, its strength lies in its geometrical overdetermination. That the 6 independent estimates of $\vec{k}(E)$ agree demonstrates that the octet model is an internally consistent model of QPI in cuprates.

Having measured $\vec{k}(E)$, how should we interpret this information? Due to the condition $\partial_{\vec{k}} \Delta_{\vec{k}} \ll \partial_{\vec{k}} \epsilon_{\vec{k}}$ being realised in cuprates, and equation $2.42, E \approx \Delta_{\vec{k}}$. For every measurement energy, the superconducting gap takes this value at the position $\vec{k}(E)$ in momentum space. Thus we can directly determine the momentum space gap function as shown in figure 2.13 .

As can be seen in figure 2.12, the tips of the banana shaped CECs lie very close to the position of the underlying Fermi surface in momentum space. Thus, by plotting all of the points $\vec{k}(E)$, one can trace out the position of the Fermi surface in momentum space as shown in figure 2.13 .

This technique will be used in chapter 4 to extract the Fermi surface geometry of $\mathrm{Bi}_{2} \mathrm{Sr}_{2} \mathrm{CaCu}_{2} \mathrm{O}_{8+\delta}$ for a range of dopings. Here, instead of using the full octet of scattering vectors, $\vec{q}_{4}$ alone is used. The set of relations 2.52 and 2.53 show that this is sufficient.

\subsection{Mapping Spatial Variations in the Amplitude and Phase of LDOS Modulations}

SI-STM spectroscopic maps contain a vast amount of information, with modulations occurring on multiple length scales and in multiple directions. Often we are only interested in the amplitude and phase of a small sub-set of these modulations. These quantities can be extracted from our data sets by filtering their complex Fourier transforms so that only wave-vectors proximal to those of interest remain. For instance, if we are interested in modulations of the spectroscopic map $M(\vec{r}, E)$ at wave-vector $\vec{Q}$ we would filter its Fourier transform $\tilde{M}(\vec{q}, E)$ to yield

$$
M_{\vec{Q}}(\vec{q}, E)=\tilde{M}(\vec{q}, E) e^{-\frac{(\vec{q}-\vec{Q})^{2}}{2 \Lambda^{2}}}
$$


(a)

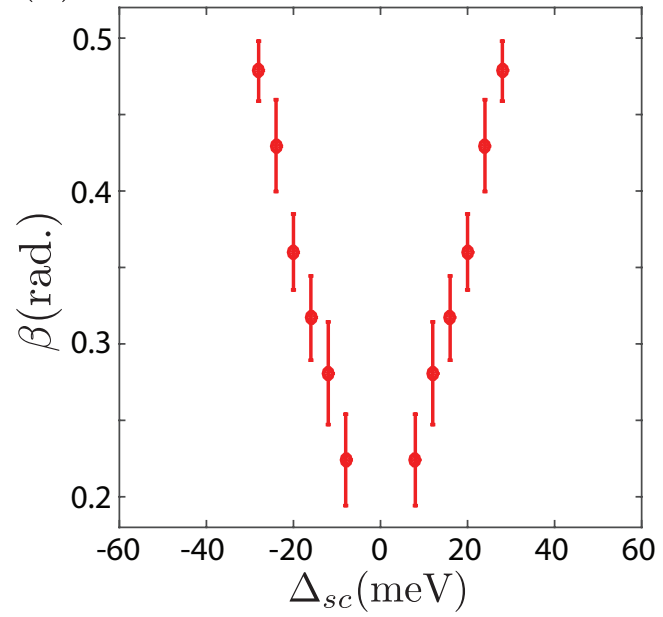

(b)

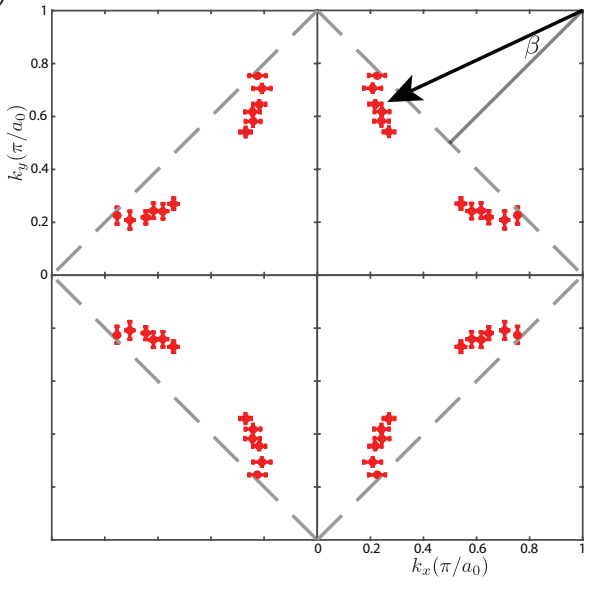

FIGURE 2.13: (a) $d$-wave superconducting gap function measured for $\mathrm{Bi}_{2} \mathrm{Sr}_{2} \mathrm{CaCu}_{2} \mathrm{O}_{8+\delta}$ with $p=17 \%$ using quasi-particle interference. Gap values are plotted against $\beta$, the angle from the nodal $(\pi, \pi)$ directions. (b) $\left(k_{x}, k_{y}\right)$ values of all points shown in (a). They trace out the underlying Fermi surface. In $\mathrm{Bi}_{2} \mathrm{Sr}_{2} \mathrm{CaCu}_{2} \mathrm{O}_{8+\delta}$ with $p<19 \%$ no quasi-particle interference is observed from quasi-particles beyond the anti-ferromagnetic Brillouin zone (dashed lines). Red points represent the mean of 6 independent $k_{x}$ and $k_{y}$ estimates made from measured wave-vectors $\vec{q}_{1}, \ldots, \vec{q}_{7}$ using equations 2.52 and 2.53 . Error bars represent the standard deviation of these 6 independent estimates.

where $\Lambda^{-1}$ is the characteristic length scale below which variations in the amplitude or phase of modulations at $\vec{Q}$ will not be resolved in this process. I will refer to this process as Fourier filtration.

Using an inverse Fourier transform one can then proceed to create the complex real-space map,

$$
M_{\vec{Q}}(\vec{r}, E)=\frac{2}{(2 \pi)^{2}} \int d \vec{q} e^{i \vec{q} \cdot \vec{r}} \tilde{M}_{\vec{Q}}(\vec{q}, E),
$$

that only contains modulations at wave-vectors proximal to $\vec{Q}$.

To make a real-space map of modulations at wave-vector $\vec{Q}$ one simply takes the amplitude of $M_{\vec{Q}}(\vec{r})$,

$$
A_{\vec{Q}}(\vec{r}, E)=\sqrt{\left(\operatorname{Re} M_{\vec{Q}}(\vec{r}, E)\right)^{2}+\left(\operatorname{Im} M_{\vec{Q}}(\vec{r}, E)\right)^{2}} .
$$

Similarly the phase of these modulations, $\phi(\vec{r})$, is given by

$$
\phi(\vec{r})=\arctan \frac{\operatorname{Im}_{\vec{Q}}(\vec{r}, E)}{\operatorname{Re} M_{\vec{Q}}(\vec{r}, E)} .
$$


In other cases we are interested in removing a specific Fourier component from an image because it is visually dominating and we wish to examine a smaller modulation at another wave-vector. In this case we can remove those modulations at wave-vectors $\pm \overrightarrow{Q^{\prime}}$ to create the real-space map

$$
M_{ \pm \vec{Q}^{\prime}}^{\prime}(\vec{r}, E)=\frac{2}{(2 \pi)^{2}} \int d \vec{q} e^{i \vec{q} \cdot \vec{r}}\left[\tilde{M}(\vec{q}, E)-\tilde{M}_{ \pm \vec{Q}^{\prime}}^{\prime}(\vec{q}, E)\right]
$$

where

$$
M_{ \pm \vec{Q}}^{\prime}(\vec{q}, E)=\tilde{M}(\vec{q}, E) \times\left[e^{-\frac{\left(\vec{q}-\vec{Q}^{\prime}\right)^{2}}{2 \Lambda^{2}}}+e^{-\frac{\left(\vec{q}+\vec{Q}^{\prime}\right)^{2}}{2 \Lambda^{2}}}\right]
$$




\section{Chapter 3}

\section{Sub-Lattice Segregated SI-STM}

\section{in Cuprates}

In this chapter I will detail novel methods, developed by myself and others in our group over the past 6 years, extending spectroscopic-imaging STM (SI-STM) to imaging states which have significant intra-unit-cell structure. I will a introduce a method of finding and segregating different atomic sites or sub-lattices. I will also outline the technical steps required to use the complex Fourier transform of SI-STM spectroscopic maps to probe intraunit-cell symmetry breaking. Focussing on cuprate superconductors, these methods will be used in chapter 4 to determine the intricate intra-unit-cell structure of the charge density wave in cuprates.

SI-STM is a real-space probe and therefore in principle retains all phase information about spatial modulations, in contrast to scattering probes. One has access to the full complex Fourier transform of conductance maps, $\tilde{g}(\vec{q}, E)$, as opposed to just the power spectrum $|\tilde{g}(\vec{q}, E)|^{2}$.

This turns out to be incredibly useful for studying the structure of electronic states within the unit cell. Consider the $\mathrm{CuO}_{2}$ plane with one copper and two oxygens per unit cell. In a perfectly periodic lattice, these sets of different atoms or sub-lattices will contribute to its Fourier transform with different phases. For instance, in cuprates if we measure the function $\operatorname{Re} \tilde{Z}\left(\left(\frac{2 \pi}{a_{0}}, 0\right), E\right)-\operatorname{Re} \tilde{Z}\left(\left(0, \frac{2 \pi}{a_{0}}\right), E\right)$, we are directly measuring only 
differences in electronic structure between the two oxygen sites in the unit cell [32]. The utility of phase-resolved SI-STM lies in this sub-lattice specificity.

In order to enact such a scheme one must remove artifacts introduced in the measurement process that distort the lattice in SI-STM spectroscopic maps away from perfect periodicity. A procedure for correcting these distortions to give a perfect lattice with the origin of co-ordinates lying on a specific atomic site, first introduced by Lawler et al. in reference [32], will be described below.

Another advantage of mapping SI-STM data to a perfectly periodic lattice is that it enables you to easily find the positions of atoms belonging to different sub-lattices. Spectroscopic maps can then be segregated into several maps, each containing only the sites of a single sub-lattice.

All of these techniques will be heavily employed in chapter 4 where they are used to make a direct detection of a $d$-symmetry form factor charge density wave in cuprates, a state where a breaking of the symmetry between the oxygen sub-lattices within the unit cell is periodically modulated in space.

As with the previous chapter, the rapid and fairly specialised development of the field means that for completeness I must touch on a fairly large number of sub-topics, some of which are fairly technical; one way to use this chapter might be to back-reference to it from the main results chapters of the thesis rather than try to take in every detail on first reading.

\subsection{Data Acquisition Parameters}

In order to implement sub-lattice segregated, phase-resolved SI-STM one requires a large number of pixels within every unit cell. To effectively discriminate between different sub-lattices we need at least three physical measurements along each $\mathrm{Cu}-\mathrm{O}-\mathrm{Cu}$ bond; a minimum of 9 pixels per $\mathrm{CuO}_{2}$ plaquette. Measurements presented in this thesis typically have between 9 and 25 pixels per unit cell. For $\mathrm{Bi}_{2} \mathrm{Sr}_{2} \mathrm{CaCu}_{2} \mathrm{O}_{8+\delta}$ this gives a pixel density $0.62-1.71 \AA^{-2}$ corresponding to between $\approx 400 \times 400$ and $650 \times 650$ measurement pixels in a typical $50 \mathrm{~nm} \times 50 \mathrm{~nm}$ field of view. 


\subsection{Atomic Phase Correction Algorithm}

I will now describe an algorithm for correcting picometer distortions in STM images caused by the non-ideality of the scanner. Such an algorithm is required to sensibly obtain intra-unit-cell information using the phase of spectroscopic map Fourier transforms and is employed throughout this thesis. This algorithm was first designed and implemented by Lawler et al. in reference [32].

Consider an atomically resolved STM topograph, $T(\vec{r})$, of a square lattice where two orthogonal wave-vectors generate the atomic corrugations. These are the reciprocal lattice vectors $\vec{Q}_{a}=\left(\vec{Q}_{a x}, \vec{Q}_{a y}\right)$ and $\vec{Q}_{b}=\left(\vec{Q}_{b x}, \vec{Q}_{b y}\right)$ with $\left|\vec{Q}_{a}\right|=\left|\vec{Q}_{b}\right|=2 \pi / a_{0}$. The ideal topographic image of this perfect square lattice can be written as

$$
T_{\text {ideal }}(\vec{r})=T_{0}\left[\cos \left(\vec{Q}_{a} \cdot \vec{r}\right)+\cos \left(\vec{Q}_{b} \cdot \vec{r}\right)\right]
$$

In SI-STM, $T(\vec{r})$ and its simultaneously measured spectroscopic current map, $I(\vec{r}, V)$, and differential conductance map, $g(\vec{r}, V)$, are measured on a square array of pixels with coordinates labeled $\vec{r}=(x, y)$. The Fourier transform of $T(\vec{r}), \tilde{T}(\vec{q})=\operatorname{Re} \tilde{T}(\vec{q})+i \tilde{T}(\vec{q})$, then exhibits two distinct peaks at $\vec{q}=\vec{Q}_{a}$ and $\vec{Q}_{b}$.

In an actual experiment, $T(\vec{r})$ suffers picometer scale distortions from the ideal representation in equation 3.1 because of the time-dependent response of the piezo-tube over the period of data acquisition ( $\sim 1$ week). This has the effect of applying a slowly varying displacement field, $\vec{u}(\vec{r})$, to the atoms in the experimental topograph $T_{\exp }(\vec{r})$. Identical distortions are also found in the spectroscopic data. Thus, a topographic image, including distortions, is schematically written as

$$
T_{\exp }(\vec{r})=T_{0}\left[\operatorname { c o s } \left(\vec{Q}_{a} \cdot(\vec{r}+\vec{u}(\vec{r}))+\cos \left(\vec{Q}_{b} \cdot(\vec{r}+\vec{u}(\vec{r}))\right]\right.\right.
$$

Then, to remove the effects of $\vec{u}(\vec{r})$ requires a local transformation $\vec{r}+\vec{u}(\vec{r}) \mapsto \vec{r}^{\prime}$ so that the corrected topograph $T_{\text {corr }}(\vec{r})=T_{\exp }\left(\vec{r}^{\prime}\right)$. If $\vec{u}(\vec{r})$ is known exactly this results in a perfect square lattice in $T_{\text {corr }}(\vec{r})$. Thus we must establish a method to reliably estimate $\vec{u}(\vec{r})$. 
It helps to recast this problem in terms of the phase of the fundamental component of atomic modulations at a given point $\vec{r}$

$$
\begin{aligned}
& \phi(\vec{r})=\vec{Q}_{a} \cdot \vec{r}+\theta_{a}(\vec{r}) \\
& \phi(\vec{r})=\vec{Q}_{a} \cdot \vec{r}+\theta_{a}(\vec{r}),
\end{aligned}
$$

where $\theta_{i}(\vec{r})=\vec{Q}_{i} \cdot \vec{u}(\vec{r})$ is an additional slowly varying phase generated by the displacement field. If there were no distortions and the $T_{\exp }(\vec{r})$ image were perfectly periodic then $\theta_{i}(\vec{r})$ would be constant. In turn this recasts equation 3.2 as

$$
T_{\exp }(\vec{r})=T_{0}\left[\cos \left(\phi_{a}(\vec{r})\right)+\cos \left(\phi_{b}(\vec{r})\right)\right]
$$

From this perspective, the two-dimensional lattice in equation 3.5 is a function of phase alone. For example, the apex of every atom in the topographic image has the same phase (modulo $2 \pi$ ) regardless of where it is in the image. When viewed in the $\vec{r}$ coordinates, the distance between such points of equal phase in the "perfect" lattice and distorted lattice is not the same. The problem of correcting $T_{\exp }(\vec{r})$ then reduces to finding a transformation to map points of equal phase in $T_{\exp }(\vec{r})$ and $T_{\text {ideal }}(\vec{r})$ onto each other. This is equivalent to finding a local transformation which makes $\theta_{a}(\vec{r})$ and $\theta_{b}(\vec{r})$ constants over all space; call them $\bar{\theta}_{a}$ and $\bar{\theta}_{b}$ respectively.

$\theta_{a}(\vec{r})$ and $\theta_{b}(\vec{r})$ are determined by a "computational lock-in" method first used by Slezak et al. [88]. $T_{\exp }(\vec{r})$ is first Fourier filtered, with filter width $\lambda$, separately for the wavevectors $\vec{Q}_{a}$ and $\vec{Q}_{b}$ to create two images $T_{a}^{\lambda}(\vec{r})$ and $T_{b}^{\lambda}(\vec{r})$. These are then multiplied by reference sine and cosine functions with periodicity set by $\vec{Q}_{a}$ and $\vec{Q}_{b}$ to yield four images

$$
\begin{aligned}
X_{a}(\vec{r}) & =T_{a}^{\lambda}(\vec{r}) \times \cos \left(\vec{Q}_{a} \cdot \vec{r}\right), Y_{a}(\vec{r})=T_{a}^{\lambda}(\vec{r}) \times \sin \left(\vec{Q}_{a} \cdot \vec{r}\right) \\
X_{b}(\vec{r}) & =T_{b}^{\lambda}(\vec{r}) \times \cos \left(\vec{Q}_{b} \cdot \vec{r}\right), Y_{b}(\vec{r})=T_{b}^{\lambda}(\vec{r}) \times \sin \left(\vec{Q}_{b} \cdot \vec{r}\right)
\end{aligned}
$$

The phases $\theta_{a}(\vec{r})$ and $\theta_{b}(\vec{r})$ are then given by

$$
\theta_{a}(\vec{r})=\arctan \frac{Y_{a}(\vec{r})}{X_{a}(\vec{r})}, \theta_{b}(\vec{r})=\arctan \frac{Y_{b}(\vec{r})}{X_{b}(\vec{r})}
$$


It is then fairly simple to construct the transformation that needs to be applied to $T_{\exp }(\vec{r})$. Let $\vec{r}$ be a point on the unprocessed $T_{\exp }(\vec{r})$ and let $\vec{r}^{\prime}$ be the point of equal phase on the perfect lattice periodic image, which needs to be determined. This produces a set of relations

$$
\begin{aligned}
& \vec{Q} \cdot \vec{r}+\theta_{a}(\vec{r})=\vec{Q}_{a} \cdot \vec{r}^{\prime}+\overline{\theta_{a}} \\
& \vec{Q} \cdot \vec{r}+\theta_{b}(\vec{r})=\vec{Q}_{b} \cdot \vec{r}^{\prime}+\bar{\theta}_{b}
\end{aligned}
$$

To solve for $u(\vec{r})$ rewrite equations 3.9 and 3.10 in matrix form

$$
\mathbf{Q}\left(\begin{array}{c}
x^{\prime} \\
y^{\prime}
\end{array}\right)=\mathbf{Q}\left(\begin{array}{c}
x \\
y
\end{array}\right)-\mathbf{Q}\left(\begin{array}{c}
\overline{\theta_{a}}-\theta_{a}(\vec{r}) \\
\overline{\theta_{b}}-\theta_{b}(\vec{r})
\end{array}\right)
$$

where

$$
\mathbf{Q}=\left(\begin{array}{cc}
Q_{a x} & Q_{a y} \\
Q_{b x} & Q_{b y}
\end{array}\right)
$$

Because $\vec{Q}_{a}$ and $\vec{Q}_{b}$ are orthogonal, $\mathbf{Q}$ is invertible allowing one to solve for the displacement field $\vec{u}(\vec{r})$ which maps $\vec{r}$ to $\vec{r}^{\prime}$ :

$$
\vec{u}(\vec{r})=\mathbf{Q}^{-1}\left(\begin{array}{c}
\overline{\theta_{a}}-\theta_{a}(\vec{r}) \\
\overline{\theta_{b}}-\theta_{b}(\vec{r})
\end{array}\right) .
$$

In practice, we use the convention $\theta_{i}=0$ which generates a "perfect" lattice with an atomic peak centered at the origin. This is equivalent to setting to zero the imaginary component of the Bragg peaks in the Fourier transform.

To construct to corrected topograph, $T_{\text {corr }}(\vec{r})$, one simply makes the assignment $T_{\text {corr }}(\vec{r})=T_{\exp }(\vec{r}+u(\vec{r}))$

\subsection{Finding Atomic Positions}

In order to examine tunnelling spectra of the individual atomic sub-lattices in a material one must first find the locations of the different species of atom in the field of view. Using the atomic phase correction procedure detailed in section 3.2 this is fairly simple in cuprates. Having mapped the topograph to a near perfect square lattice and having 
set the origin of co-ordinates on an atomic site, we know that apices of the atoms in the topograph appear at points where $\vec{Q}_{a} \cdot \vec{r}=0 \bmod 2 \pi$ and $\vec{Q}_{b} \cdot \vec{r}=0 \bmod 2 \pi$.

In $\mathrm{Bi}_{2} \mathrm{Sr}_{2} \mathrm{CaCu}_{2} \mathrm{O}_{8+\delta}$ the cleavage plane is a $\mathrm{BiO}$ surface and the atomic contrast originates from $\mathrm{Bi}$ atoms. It has been shown that the copper sites in the $\mathrm{CuO}_{2}$ plane are registered to the $\mathrm{Bi}$ atoms to within a phase accuracy of $2 \%$ of $2 \pi$ [89]. Thus in spectroscopic maps we identify the positions of copper $(\mathrm{Cu})$, x-directed oxygen $\left(\mathrm{O}_{x}\right)$ and y-directed oxygen $\left(\mathrm{O}_{y}\right)$ using the criteria

$$
\vec{r} \in\left\{\begin{array}{llll}
\vec{r}_{C u} & \theta_{a}=0 & \bmod 2 \pi \text { and } \theta_{b}=0 & \bmod 2 \pi \\
\vec{r}_{O_{x}} & \theta_{a}=\pi & \bmod 2 \pi \text { and } \theta_{b}=0 & \bmod 2 \pi \\
\vec{r}_{O_{y}} & \theta_{a}=0 & \bmod 2 \pi \text { and } \theta_{b}=\pi & \bmod 2 \pi
\end{array}\right.
$$

In $\mathrm{Na}_{x} \mathrm{Ca}_{2-x} \mathrm{CuO}_{2} \mathrm{Cl}_{2}$ the atoms primarily giving rise to atomic contrast in topography are chlorine which, again, are positioned above the copper atoms in the $\mathrm{CuO}_{2}$ plane. As such the criteria listed in equation 3.14 are also used for $\mathrm{Na}_{x} \mathrm{Ca}_{2-x} \mathrm{CuO}_{2} \mathrm{Cl}_{2}$.

\subsection{Sub-Lattice Segregation}

Once lists of the positions of atoms lying in different sub-lattices have been constructed as detailed in section 3.3, we can segregate maps according to sub-lattice specific maps. From the three lists of atomic co-ordinates $\left\{\vec{r}_{C u}\right\},\left\{\vec{r}_{O_{x}}\right\}$ and $\left\{\vec{r}_{O_{y}}\right\}$, three masking images, $S_{C u}(\vec{r}), S_{O_{x}}(\vec{r})$ and $S_{O_{y}}(\vec{r})$ are created. These are discrete, having the same number of pixels as the map we wish to segregate into sub-lattices, $M(\vec{r})$.

For each of the elements in $\left\{\vec{r}_{C u}\right\}$, the closest pixel in $S_{C u}(\vec{r})$ is assigned the value 1 and all other pixels the value zero. The same process is carried out for $\left\{\vec{r}_{O_{x}}\right\}$ and $S_{O_{x}}$; $\left\{\vec{r}_{O_{y}}\right\}$ and $S_{O_{y}}$. To minimise the mismatch between the true atomic positions and the pixel positions, the maps can be interpolated to higher pixel density.

If we wish to select more than 1 pixel around each atom, the isolated non-zero values in $S_{C u}(\vec{r}), S_{O_{x}}(\vec{r})$ and $S_{O_{y}}(\vec{r})$ can be replaced with appropriately sized square blocks of ones. Typically between 1 and 9 pixels are used per atom. 
The sub-lattice segregated functions $C u(\vec{r}), O_{x}(\vec{r})$ and $O_{y}(\vec{r})$ are then given by

$$
\begin{aligned}
& C u(\vec{r})=S_{C u}(\vec{r}) M(\vec{r}) \\
& O_{x}(\vec{r})=S_{O_{x}}(\vec{r}) M(\vec{r}) \\
& O_{y}(\vec{r})=S_{O_{y}}(\vec{r}) M(\vec{r}) .
\end{aligned}
$$
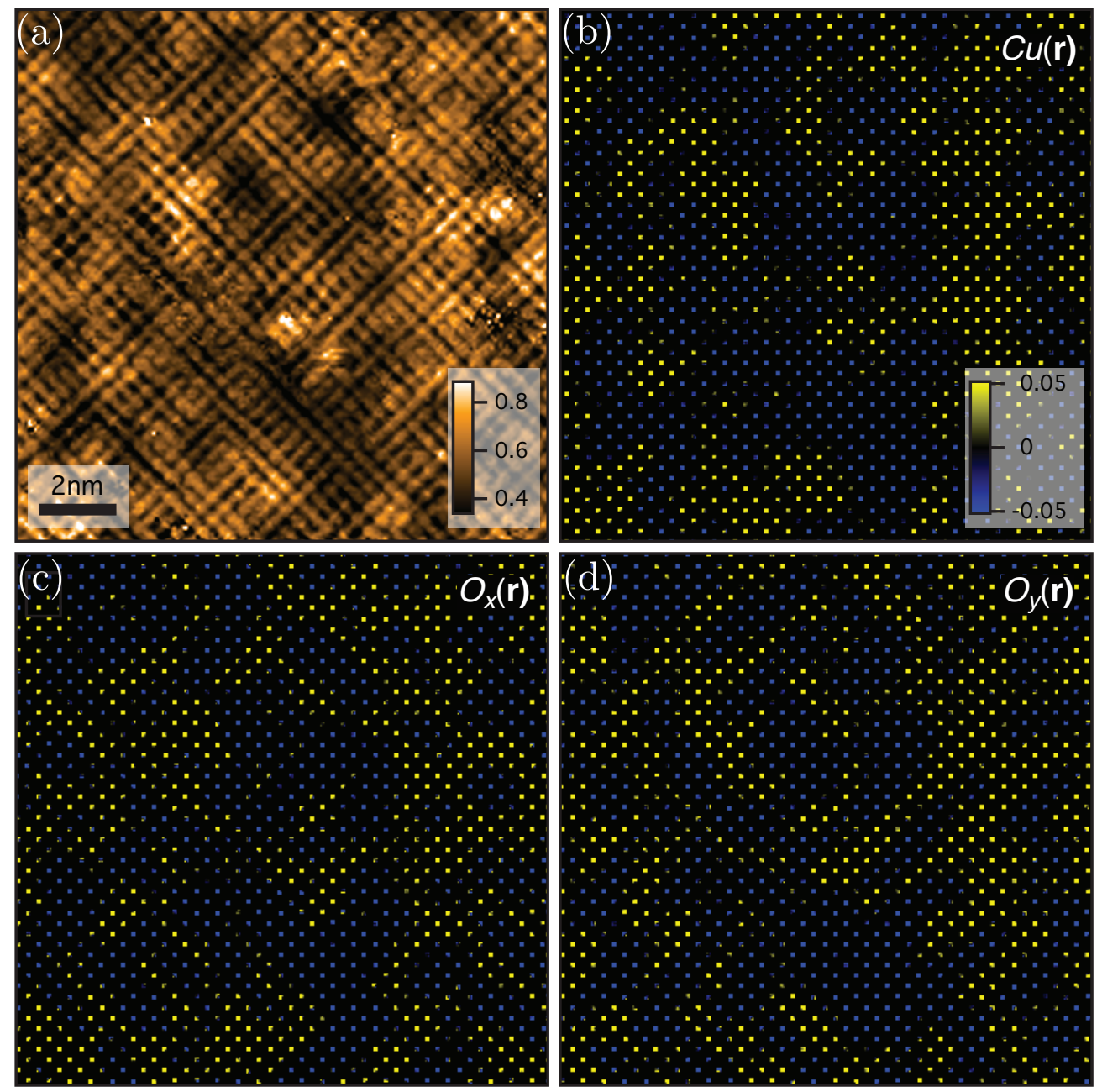

Figure 3.1: (a) Measured $Z(\vec{r},|E|=150 \mathrm{mV})$ for an $\mathrm{Na}_{x} \mathrm{Ca}_{2-x} \mathrm{CuO}_{2} \mathrm{Cl}_{2}$ sample with $p \sim 12 \pm 1 \%$. (b) Copper sub-lattice segregated image, $C u(\vec{r})$, with copper sites selected from (a). (c) x-direction oxygen sub-lattice segregated image, $O_{x}(\vec{r})$, with x-oxygen sites selected from (a). (d) y-directed oxygen sub-lattice segregated image, $O_{y}(\vec{r})$, with y-oxygen sites selected from (a). The spatial mean has been subtracted from (b)-(d).

Figure 3.1 (a) shows $Z(\vec{r},|E|=150 \mathrm{mV})$ for an $\mathrm{Na}_{x} \mathrm{Ca}_{2-x} \mathrm{CuO}_{2} \mathrm{Cl}_{2}$ sample with $p \sim 12 \pm$ 1\%. In figures 3.1 (b)-(d) I show sub-lattice segregated images $C u(\vec{r}), O_{x}(\vec{r})$ and $O_{y}(\vec{r})$ derived from (a). 


\subsection{Intra-Unit-Cell Spatial Registration of Measurements}

Sometimes one would like to examine how the intra-unit-cell electronic structure changes as a function of a tuning parameter, such as setup bias or magnetic field. To do this we must register data sets, measured in the same field-of-view under identical conditions apart from the change in tuning parameter, and register them to each other with picometer accuracy. Unfortunately this is not possible to implement directly in the measurement because of time-dependent variations in the response of the piezo-tube to applied voltages. However, this can be implemented at the data analysis stage.

For the two data sets to be registered, we will use their topographs, $T_{1}(\vec{r})$ and $T_{2}(\vec{r})$, to align identical atoms in one data set with those in the other. The algorithm is as follows:

1. Apply the phase-correction algorithm detailed in section 3.2 to both $T_{1}(\vec{r})$ and $T_{2}(\vec{r})$ to remove picometer distortions of the image introduced by non-ideality of the piezo-tube.

2. $T_{1}(\vec{r})$ and $T_{2}(\vec{r})$ are then roughly aligned. This corrects for any small difference in field-of-view between the measurements. It is implemented through an optimisation process where rigid spatial translations are applied to $T_{2}(\vec{r})$ to yield $T_{2}^{\prime}(\vec{r})$. The parameter that is optimised is the cross-correlation of $T_{2}^{\prime}(\vec{r})$ and $T_{1}(\vec{r})$.

3. For both $T_{2}^{\prime}(\vec{r})$ and $T_{1}(\vec{r})$ the positions of the $\mathrm{Cu}, \mathrm{O}_{x}$ and $\mathrm{O}_{y}$ atoms are determined. The positions of atoms $1 \ldots$ i in $T_{1}(\vec{r})$ form a set $\left\{\vec{r}_{i}{ }^{1}\right\}$. The identical set of atoms in $T_{2}^{\prime}(\vec{r})$ have positions $\left\{\vec{r}_{i}^{2}\right\}$. The positions $\left\{\vec{r}_{i}^{2}\right\}$ are used to form a mesh by Delaunay triangulation. Then, an affine transformation within each triangle of the mesh is applied to move the points $\left\{\vec{r}_{i}^{2}\right\}$ to positions $\left\{\vec{r}_{i}^{1}\right\}$, creating a topograph $T_{2}^{\prime \prime}(\vec{r})$.

4. After this process, the atoms in topographs $T_{1}(\vec{r})$ and $T_{2}^{\prime \prime}(\vec{r})$ are registered with an accuracy determined by the the filter size $\lambda$ used in step 1, typically picometers (see section 3.2).

5. All transformations applied to $T_{2}(\vec{r})$ and $T_{2}^{\prime}(\vec{r})$ are then applied to the simultaneously acquired spectroscopic maps. 
Two registered topographs of a $\mathrm{Bi}_{2} \mathrm{Sr}_{2} \mathrm{CaCu}_{2} \mathrm{O}_{8+\delta}$ surface taken with $\mathrm{B}=8.25 \mathrm{~T}$ and $\mathrm{B}=0 \mathrm{~T}$ respectively, registered with picometer accuracy, are shown in figure 3.2.

(a)

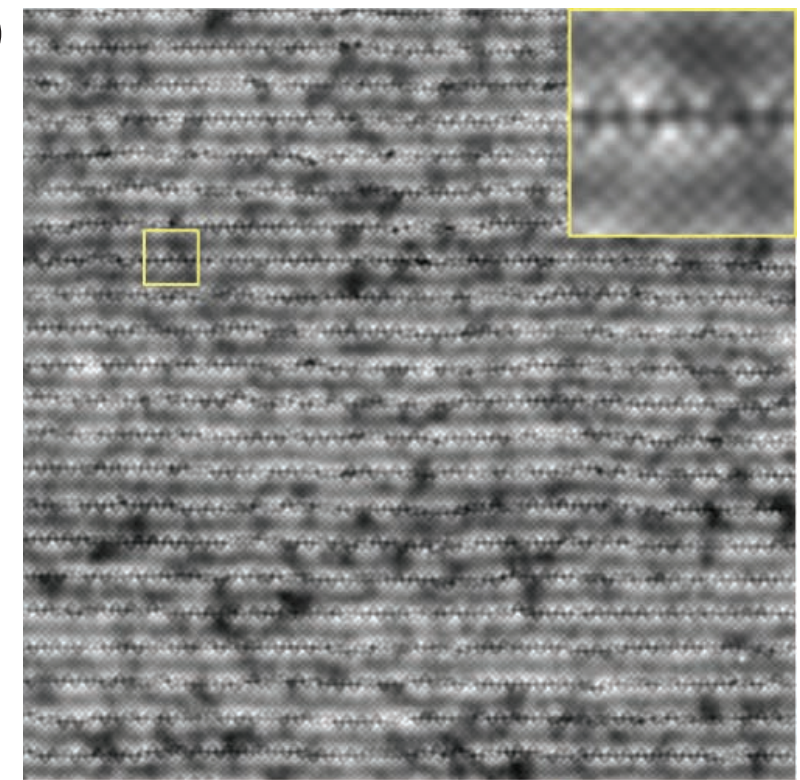

(b)

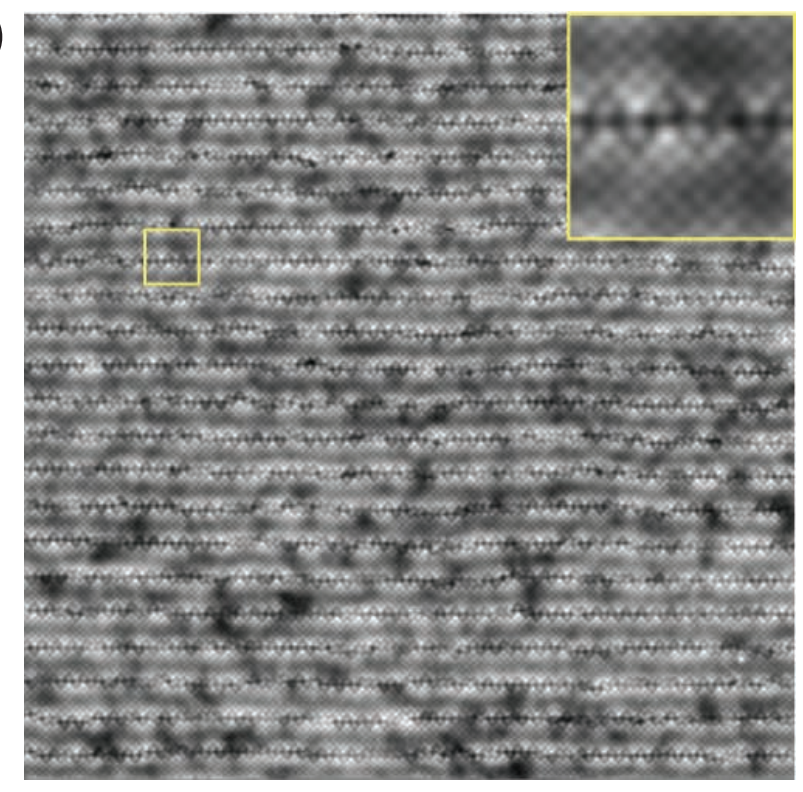

Figure 3.2: Spatially registered topographs of $\mathrm{Bi}_{2} \mathrm{Sr}_{2} \mathrm{CaCu}_{2} \mathrm{O}_{8+\delta}$ taken at (a) $\mathrm{B}=8.25 \mathrm{~T}$ and $(\mathrm{b}) \mathrm{B}=0 \mathrm{~T}$. The images show a $65 \mathrm{~nm} \times 65 \mathrm{~nm}$ region of the sample and are registered to within $10 \mathrm{pm}$. Insets show magnified images of the region indicated by the yellow squares. 



\section{Chapter 4}

\section{Atomic-scale Electronic Structure of the Cuprate $d$-symmetry Form Factor Charge Density Wave}

The results of this chapter are reported in "Direct Phase-Sensitive Identification of a $d$-Form Factor Density Wave in Underdoped Cuprates", Proceedings of the National Academy of Sciences, 111, E3026 (2014) and "Atomic-Scale Electronic Structure of the Cuprate $d$-Symmetry Form Factor Density Wave State", Nature Physics, 12, 150 (2016).

This chapter details the use of sub-lattice segregated spectroscopicimaging STM to reveal the intra unit cell electronic structure of the charge density wave in the cuprate superconductors $\mathrm{Bi}_{2} \mathrm{Sr}_{2} \mathrm{CaCu}_{2} \mathrm{O}_{8+\delta}$ and $\mathrm{Na}_{x} \mathrm{Ca}_{2-x} \mathrm{CuO}_{2} \mathrm{Cl}_{2}$.

In chapter 1 I reviewed recent experimental evidence demonstrating that unconventional density wave order is a generic feature of cuprate superconductors. Many authors have predicted that the charge density wave (CDW) in cuprates should exhibit a $d$-symmetry form factor, characterised by the modulation of a breaking of intra unit cell rotational symmetry [90-106]. In this chapter I will demonstrate that the short-ranged CDW order in $\mathrm{Bi}_{2} \mathrm{Sr}_{2} \mathrm{CaCu}_{2} \mathrm{O}_{8+\delta}$ and $\mathrm{Na}_{x} \mathrm{Ca}_{2-x} \mathrm{CuO}_{2} \mathrm{Cl}_{2}$ has a predominantly $d$-symmetry form factor, hitherto unobserved in nature. 
While CDW order appears to be ubiquitous amongst cuprate materials, the mechanism of its formation, which lattice symmetries it breaks, and its relationship to the pseudogap and high-temperature superconductivity are unknown. Furthermore, little is understood about how the CDW is derived from or imparts itself upon the electronic structure of the $\mathrm{CuO}_{2}$ plane. In this chapter I will utilise the development of sub-lattice segregated spectroscopic-imaging STM (SI-STM), detailed in chapter 3, to address these questions.

\subsection{Form Factor Decomposition of Modulations in the $\mathrm{CuO}_{2}$ Plane}

In this chapter I will report on the detection of a predominantly $d$-symmetry form factor CDW in cuprates. Such a CDW consists of an intra-unit-cell breaking of rotational symmetry that is modulated periodically in space. In cuprates, the $\mathrm{CuO}_{2}$ plane has three sub-lattices (figure 4.1): copper sites at positions $\left\{\vec{r}_{C u}\right\}$, x-directed oxygen sites at positions $\left\{\vec{r}_{O_{x}}\right\}$ and y-directed oxygen sites at positions $\left\{\vec{r}_{O_{y}}\right\}$.

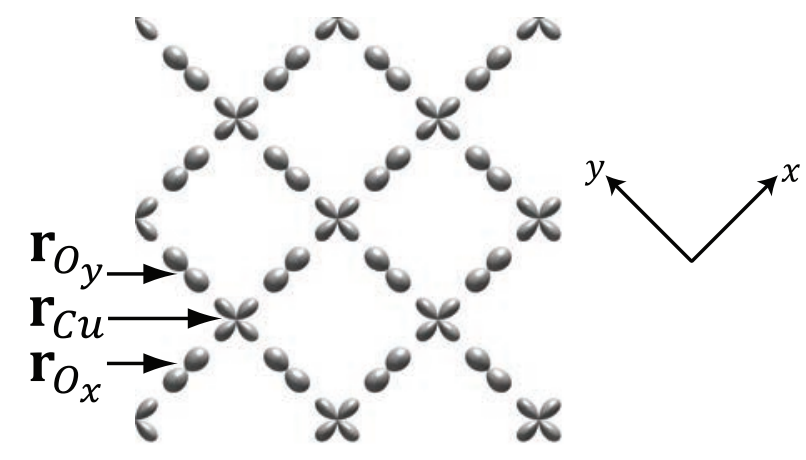

Figure 4.1: $\mathrm{Cu}, \mathrm{O}_{x}$ and $\mathrm{O}_{y}$ orbitals, on the three sub-lattices in the $\mathrm{CuO}_{2}$ plane; $\vec{r}_{C u}$ sub-lattice contains only the $\mathrm{Cu}$ sites, $\vec{r}_{O_{x}}$ sub-lattice contains only the $\mathrm{O}_{x}$ sites, and $\vec{r}_{O_{y}}$ sub-lattice contains only the $O_{y}$ sites.

Density waves consisting of modulations on the $\mathrm{x}$-directed oxygen sites $\left(\mathrm{O}_{x}\right)$ that are distinct from those on the y-directed oxygen sites $\left(\mathrm{O}_{y}\right)$ can be challenging to conceptualise. Among the many ways of organising density waves in the $\mathrm{CuO}_{2}$ plane, one is to think of them as a modulation on the copper sites $(\mathrm{Cu})$, a modulation on the $\mathrm{O}_{x}$ sites, and a modulation on the $\mathrm{O}_{y}$ sites. The results presented in this chapter, however, present a compelling case that another organisation captures the density wave observed 
in a remarkably simple way. This way organises them by form factors that I will call $s$, $s^{\prime}$ and $d$.

The wave-vector of a CDW itself breaks rotational symmetry. This means that the form factor of a CDW does not alone determine its rotational symmetries. Consequently there is a freedom in the choice of form factor definition. Here I adopt the convention introduced by Sachdev and La Placa in reference [99] which is detailed in appendix B. In the discussion below I will refer to spatial modulations of a density $\rho(\vec{r})$. This density could be any density-like observable (i.e., invariant under time-reversal and spin rotations), such as charge or local density of states.

We can think of the form factor organisation as a modulation of intra-unit-cell $(\vec{q}=0)$ electronic configurations whose point group symmetry is well defined, as shown in figure 4.2. The $\vec{q}=0 s$-symmetry form factor has a density,

$$
\rho\left(\vec{r}_{C u}\right)=A_{s}, \rho\left(\vec{r}_{O_{x}}\right)=0, \rho\left(\vec{r}_{O_{y}}\right)=0
$$

the $\vec{q}=0 s^{\prime}$-symmetry form factor has density,

$$
\rho\left(\vec{r}_{C u}\right)=0, \rho\left(\vec{r}_{O_{x}}\right)=A_{s^{\prime}}, \rho\left(\vec{r}_{O_{y}}\right)=A_{s^{\prime}}
$$

and the $\vec{q}=0 d$-symmetry form factor has density,

$$
\rho\left(\vec{r}_{C u}\right)=0, \rho\left(\vec{r}_{O_{x}}\right)=A_{D}, \rho\left(\vec{r}_{O_{y}}\right)=-A_{D}
$$

As these three intra-unit-cell configurations preserve lattice translational symmetry, they correspond to specific representations of the point group symmetry of the lattice ${ }^{1}$. Phaseresolved Fourier transforms of each arrangement could reveal their point group symmetry from the relative signs of the Bragg amplitudes. The $s$ - and $s$-symmetry form factors both share $90^{\circ}$ rotational symmetry in their Bragg-peak amplitudes, whereas the Braggpeak amplitudes for a $d$-symmetry form factor change sign under $90^{\circ}$ rotations as shown in figure 4.2. Thus, by studying the magnitude and sign of the Bragg amplitudes in

\footnotetext{
${ }^{1}$ Note that $s$ - and $s$-symmetry form factors both transform as the trivial representation of the point group and thus, even at $\vec{q}=0$, are not symmetry distinct.
} 
phase-resolved sub-lattice segregated SI-STM images, one can extract the degree to which any translationally invariant intra-unit-cell arrangement has an $s$, an $s$, or a $d$-symmetry form factor.

The intra-unit-cell $(\vec{q}=0)$ nematic previously reported by phase-resolved STM measurements in underdoped cuprates, in this description, consists of an admixture of all three of these form factors $[29,32,89]$. The presence of a non-zero $d$-symmetry component is that which indicates a breaking of the point group symmetry.
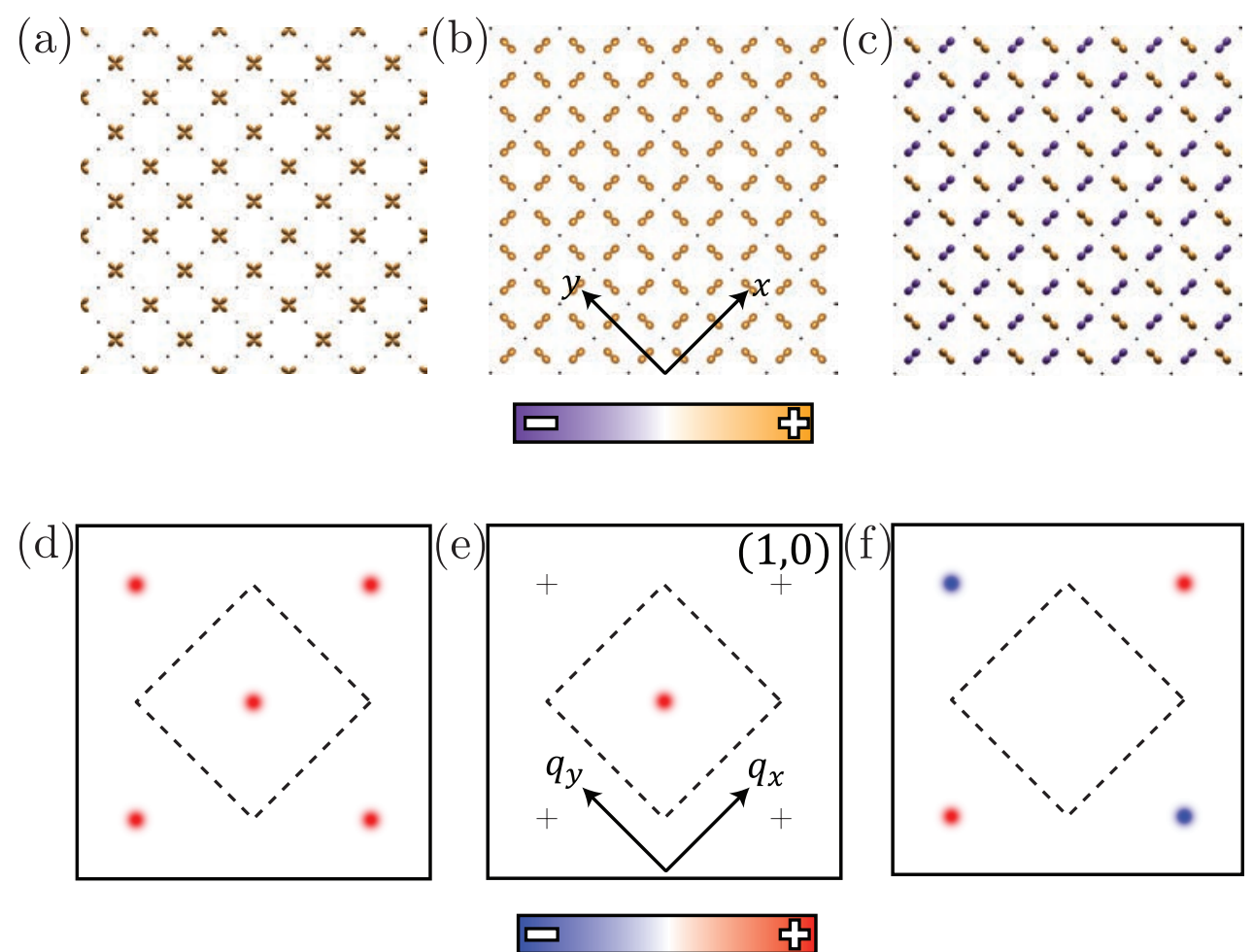

Figure 4.2: (a) Schematic of uniform density on the Cu atoms ( $s$-symmetry). The inactive $\mathrm{O}$ sites are now indicated by black dots. (b) Schematic of uniform density on the $\mathrm{O}$ atoms (also an $s$-symmetry referred to here as extended-s or $s$-symmetry). The inactive $\mathrm{Cu}$ sites are indicated by black dots. (c) Schematic pattern with oppositesign density on $\mathrm{O}_{x}$ and $\mathrm{O}_{y}$ (d-symmetry). The inactive $\mathrm{Cu}$ sites are indicated by black dots. (d) Real component of Fourier transform of the $s$-symmetry intra-unit-cell patterns derived only from $\mathrm{Cu}$ sub-lattice in (a) and with no CDW modulation. The Bragg peaks have the same sign, indicating the intra-unit-cell states have $s$-symmetry. (e) Real component of Fourier transform of the $s$ 'symmetry intra-unit-cell patterns derived only from $\mathrm{O}_{x}$ and $\mathrm{O}_{y}$ sub-lattices in (b) and with no CDW. The only Bragg peaks within the $\mathrm{CuO}_{2}$ reciprocal unit cell is at $(0,0)$. (f) Real component of Fourier transform of the $d$-symmetry intra-unit-cell patterns derived only from $\mathrm{O}_{x}$ and $\mathrm{O}_{y}$ sublattices as shown in (c) and with no CDW modulation. The Bragg peaks now have the opposite sign, indicating the intra-unit-cell states have $d$-symmetry.

Modulating these form factors at $\vec{Q} \neq 0$, we then obtain, 


$$
\begin{aligned}
& \rho_{s}(\vec{r})= \begin{cases}A_{s} \cos \left(\vec{Q} \cdot \vec{r}+\phi_{s}\right), & \vec{r}=\vec{r}_{C u} \\
0, & \vec{r}=\vec{r}_{O_{x}} \\
0, & \vec{r}=\vec{r}_{O_{y}}\end{cases} \\
& \rho_{s^{\prime}}(\vec{r})= \begin{cases}0, & \vec{r}=\vec{r}_{C u} \\
A_{s^{\prime}} \cos \left(\vec{Q} \cdot \vec{r}+\phi_{s^{\prime}}\right), & \vec{r}=\vec{r}_{O_{x}} \\
A_{s^{\prime}} \cos \left(\vec{Q} \cdot \vec{r}+\phi_{s^{\prime}}\right), & \vec{r}=\vec{r}_{O_{y}}\end{cases} \\
& \rho_{D}(\vec{r})= \begin{cases}0, & \vec{r}=\vec{r}_{C u} \\
A_{D} \cos \left(\vec{Q} \cdot \vec{r}+\phi_{D}\right), & \vec{r}=\vec{r}_{O_{x}} \\
-A_{D} \cos \left(\vec{Q} \cdot \vec{r}+\phi_{D}\right), & \vec{r}=\vec{r}_{O_{y}}\end{cases}
\end{aligned}
$$

where $\phi_{S, S^{\prime}, D}$ are the phases of each of the CDW form factor components. If, for simplicity, we choose $\phi_{S}=\phi_{S^{\prime}}=\phi_{D}=\phi(\vec{r})$ and allow for spatial disorder of the phase of the CDW we arrive at

$$
\rho(\vec{r})=\left[S(\vec{r})+S^{\prime}(\vec{r})+D(\vec{r})\right] \cos (\vec{Q} \cdot \vec{r}+\phi(\vec{r})),
$$

with $S(\vec{r})=A_{S}$ if $\vec{r} \in \vec{r}_{C u}$ and otherwise zero; $S^{\prime}(\vec{r})=A_{S^{\prime}}$ if $\vec{r} \in \vec{r}_{O_{x}}$ or $\vec{r}_{O_{y}}$ and otherwise zero; $D(\vec{r})=A_{D}$ if $\vec{r} \in \vec{r}_{O_{x}},-A_{D}$ if $\vec{r} \in \vec{r}_{O_{y}}$ and otherwise zero.

Switching back to the individual sub-lattice picture we can see that an $s$-symmetry form factor CDW consists of a modulation of the $\mathrm{Cu}$ sites alone. An $s$ '-symmetry form factor CDW will modulate the $\mathrm{O}_{x}$ and $\mathrm{O}_{y}$ sites with equal amplitude and in-phase. In the case of a $d$-symmetry form factor CDW, the $\mathrm{O}_{x}$ and $\mathrm{O}_{y}$ sites are modulated with equal amplitude but in anti-phase. This $\pi$ phase shift between the oxygen sub-lattices is the essence of the $d$-symmetry form factor and encodes the local breaking of rotation symmetry. Cartoons of these different form factor modulations are shown figures 4.3 (a)-(c).

A generic CDW can actually have $S, S^{\prime}$, and $D$ all nonzero because the directionality of the modulation wave-vector $\vec{Q}$ breaks rotational symmetry itself. Therefore, to measure the form factor components of a CDW, one should consider the structure of its Fourier 
transform. Figures 4.3 (d)-(f) show the Fourier transforms for $s^{-}, s^{\prime}$ and $d$-symmetry form factor CDWs respectively.

For an $s$-symmetry form factor CDW with wave-vector $\vec{Q}_{x}$, the Fourier transform (figure 4.3 (d)) will exhibit peaks at the primary wave-vectors $\pm \vec{Q}_{x}$, as well as Bragg-satellites $\vec{Q}^{\prime}=(1,0) \pm \vec{Q}_{x}$ and $\vec{Q}^{\prime \prime}=(0,1) \pm \vec{Q}_{x}$. In the absence of disorder all peaks will have the same sign. An $s$ '-symmetry form factor CDW will also have peaks the primary wave-vector $\pm \vec{Q}_{x}$, but not at the Bragg-satellites (figure 4.3 (e)).

A $d$-symmetry form factor has modulations on both oxygen sub-lattices but with a $\pi$ phase shift between them. This leads to a cancellation of the peaks at the primary wave-vectors $\pm \vec{Q}_{x}$ within the first Brillouin zone (dashed box figure 4.3 (f)). However, there will be peaks at the Bragg satellites $\vec{Q}^{\prime}=(1,0) \pm \vec{Q}_{x}$ and $\vec{Q}^{\prime \prime}=(0,1) \pm \vec{Q}_{x}$ but with a sign change between them. An equivalent effect is seen for the $\vec{q}=0$ case in figure $4.2(\mathrm{f})$.

These distinct patterns of peaks in the Fourier transforms allow us to measure the relative proportion of each form factor component in a CDW. A strategy for doing so using sub-lattice segregated SI-STM will be discussed in section 4.3, with specific emphasis on the experimental signatures of a predominantly $d$-symmetry form factor CDW. The next section will discuss theoretical proposals for why a cuprate CDW might possess this quality.

\subsection{Proposals for a $d$-Symmetry Form Factor CDW in Cuprates}

Many recent theories of a cuprate CDW predict that it should exhibit a predominantly $d$-symmetry form factor [90-106]. These theories can be assigned to one of three broad categories with regard to their physical reason for the predominance of the $d$-symmetry form factor.

One class of models used to consider a cuprate CDW are the spin-fermion "hot-spot" models [94-97, 101, 103, 104]. In these models a complete hole-like Fermi surface arising from an effective one-band model of the $\mathrm{CuO}_{2}$ plane is coupled to anti-ferromagnetic spin fluctuations. The effective interaction between fermions can lead to both particle-particle 

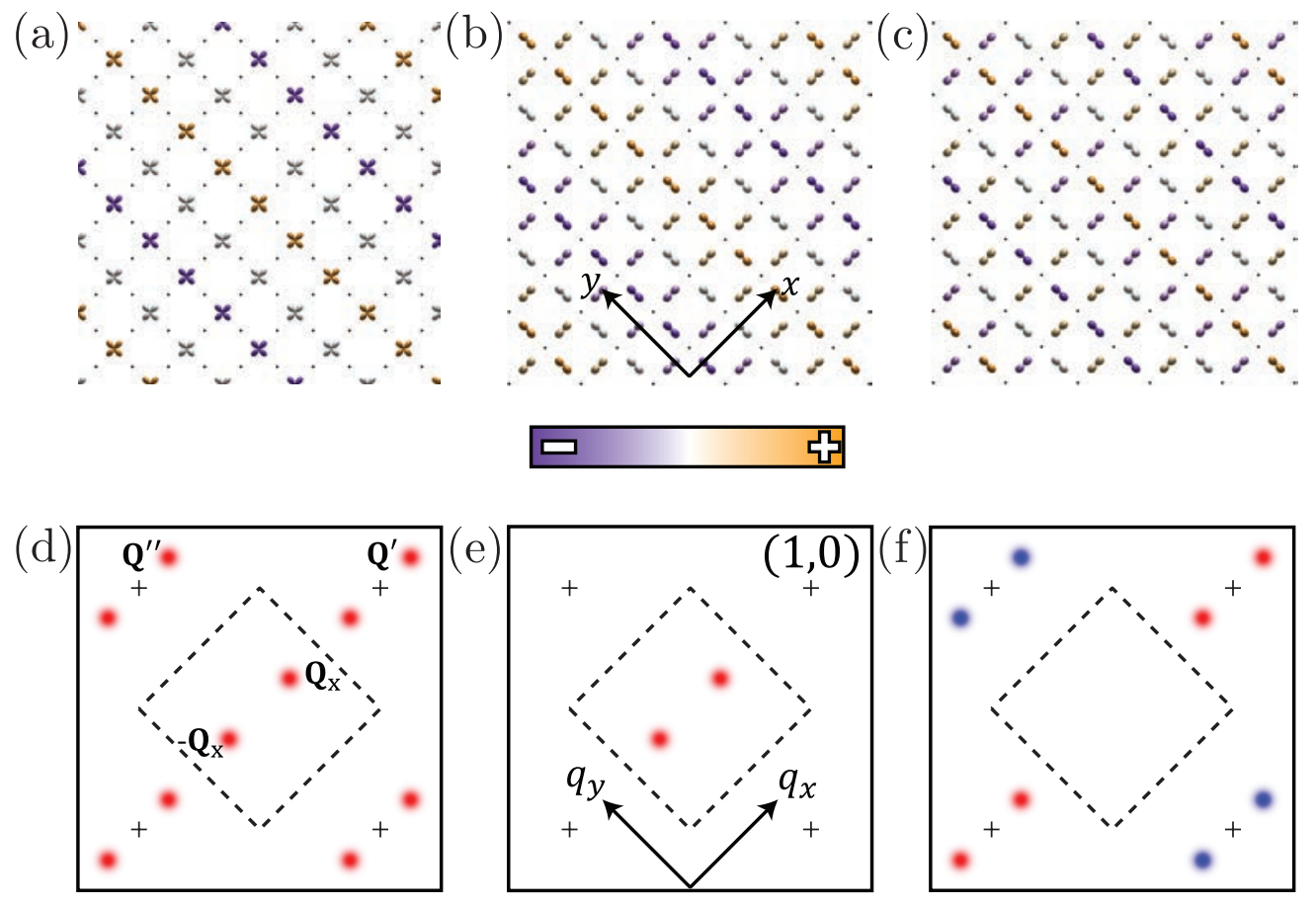

口 फ

Figure 4.3: (a) Spatial modulation with wave-vector $\vec{Q}=(Q, 0)$ of the $s$-symmetry intra-unit-cell patterns in figure 4.2 (a) is described by $\rho_{S}(\vec{r})=S(\vec{r}) \cos (\vec{Q} \cdot \vec{r})$; only $\mathrm{Cu}$ sites are active. The inactive $\mathrm{O}$ sites are indicated by black dots. (b) Spatial modulation with wave-vector $\vec{Q}$ of the patterns in figure 4.2 (b) described by $\rho_{S}^{\prime}(\vec{r})=S^{\prime}(\vec{r}) \cos (\vec{Q} \cdot \vec{r})$; only $\mathrm{O}_{x}$ and $\mathrm{O}_{y}$ sites are active but they are always equivalent within each unit cell. The inactive $\mathrm{Cu}$ sites are indicated by black dots. (c) Spatial modulation with wavevector $\vec{Q}$ of the patterns in figure 4.2 (c) described by $\rho_{D}(\vec{r})=D(\vec{r}) \cos (\vec{Q} \cdot \vec{r})$; only $\mathrm{O}_{x}$ and $\mathrm{O}_{y}$ sites are relevant but now they are always inequivalent and indeed $\pi$ out of phase. (d) $\operatorname{Re} \tilde{\rho}_{S}(\vec{q})$, the real component of Fourier transform of the pattern in (a). For this $s$-form factor CDW, the CDW satellites of Bragg peaks $\vec{Q}^{\prime}$ and $\vec{Q}^{\prime \prime}$ exhibit the same sign. (e) $\operatorname{Re} \tilde{\rho}_{S^{\prime}}(\vec{q})$, the real component of Fourier transform of the pattern in (b). For this $s^{\prime}$-form factor CDW, the peaks at $\vec{Q}$ are clear but there are no Bragg-satellites at $\vec{Q}^{\prime}$ and $\vec{Q}^{\prime \prime}$. (f) $\operatorname{Re} \tilde{\rho}_{D}(\vec{q})$, the real component of Fourier transform of the pattern in (c). For this $d$-symmetry form factor CDW, the CDW Bragg-satellite peaks at $\vec{Q}^{\prime}$ and $\vec{Q}^{\prime \prime}$ exhibit opposite sign. More profoundly, because they are out of phase by $\pi$ the contributions of $\mathrm{O}_{x}$ and $\mathrm{O}_{y}$ sites in each unit cell cancel, resulting in the disappearance of the CDW modulation peaks $\vec{Q}$ within the Brillouin zone (dashed box). 
and particle-hole instabilities. The former is an instability to $d_{x^{2}-y^{2}}$ superconductivity and the latter an instability towards a CDW with a predominantly $d$-symmetry form factor [94]. In both cases the $d$-wave character of the order arises from the momentum dependence of the effective interaction. The effective interaction is maximal and repulsive on-site, but attractive on nearest neighbour sites [107]. This naturally leads to $d$-wave like ordering.

Another potential physical reason for a predominantly $d$-symmetry form factor CDW in cuprates is coupling to a phonon with $\mathrm{d}_{x^{2}-y^{2}}$ character [92, 93]. Such a scenario is supported by "kinks" in the band dispersion, as determined by ARPES, associated with coupling to a $B_{1 g}$ phonon [108] as well as the softening of phonon modes at the CDW wave-vector in $\mathrm{YBa}_{2} \mathrm{Cu}_{3} \mathrm{O}_{6+x}[109]$.

The third scenario is that the predominantly $d$-symmetry form factor of the CDW arises as a result of the Coulomb interaction within the unit cell $[98,102,105,106]$. The large on-site Coulomb repulsion (Hubbard $\mathrm{U}$ ) for the $\mathrm{Cu} 3 \mathrm{~d}$ orbitals acts to suppress charge modulations on this site, naturally favouring a modulation in the occupation of oxygen $2 \mathrm{p}_{x, y}$ orbitals instead (an $s^{\prime}$ or $d$-symmetry form factor). The Coulomb interaction between the $\mathrm{x}$ and $\mathrm{y}$ directed oxygen sites will then favour a predominantly $d$-symmetry from factor CDW. Additionally this would also minimise the net charge accumulation within the unit cell and hence reduce the long range coulomb interaction between unit cells [110].

\subsection{SI-STM Signatures of a $d$-Symmetry Form Factor CDW}

With the recent development of STM techniques to image intra-unit-cell electronic structure detailed in chapter $3[27,29,32,89]$, it was suggested by Subir Sachdev that a practical approach to determining the form factor of underdoped cuprate CDWs would be to partition each such image of the $\mathrm{CuO}_{2}$ electronic structure into three separate images. The first image contains only the measured values at $\mathrm{Cu}$ sites, $\mathrm{Cu}(\vec{r})$, and the other two images $O_{x}(\vec{r})$ and $O_{y}(\vec{r})$ contain only the measurements at the $\mathrm{x} / \mathrm{y}$-directed oxygen sites. Once the original electronic structure image is thus separated, the phase-resolved 
Fourier transform of $C u(\vec{r}), O_{x}(\vec{r})$ and $O_{y}(\vec{r})\left(\tilde{C u}(\vec{q}), \tilde{O_{x}}(\vec{q}), \tilde{O}_{y}(\vec{q})\right)$ may, in principle, be used to reveal the form factor of a CDW.

Figure 4.4 (a) shows a $\vec{q}=0$ state with a $d$-symmetry form factor, a state known as an intra-unit-cell nematic. Its phase-resolved Fourier transform (figure 4.4 (b)) has a sign change between Bragg peaks that are related by $90^{\circ}$ rotations. In figure 4.4 (c) this $\vec{q}=0$ state is modulated at the wave-vector $\vec{Q}=\vec{Q}_{x}$ to give a $d$-symmetry form factor CDW. By considering the two trajectories parallel to $\vec{Q}_{x}$ marked $\mathrm{O}_{x}$ and $\mathrm{O}_{y}$, we see that the amplitude of the wave on $\mathrm{O}_{x}$ is exactly $\pi$ out of phase with that along the adjacent trajectory on $\mathrm{O}_{y}$. For this reason, when its Fourier transform is determined in figure 4.4 (d), no peaks occur at the primary wave-vectors $\pm \vec{Q}_{x}$, inside the first Brillouin zone (dashed box). The second distinctive feature of the Fourier transform is that the Bragg satellite peaks at $\vec{Q}^{\prime}=(1,0) \pm \vec{Q}_{x}$ and $\vec{Q}^{\prime \prime}=(0,1) \pm \vec{Q}_{x}$ have opposite sign.

A $d$-symmetry form factor CDW with modulations along both $\mathrm{x}$ and y axes at $\vec{Q}=$ $(Q, 0) ;(0, Q)$ should then exhibit two key characteristics exemplified by figure 4.4 (e) whose equivalent experimental information is contained in $\operatorname{Re} \tilde{O}_{x}(\vec{q})+\operatorname{Re} \tilde{O_{y}}(\vec{q})$. The first is that modulation peaks at $\pm \vec{Q}$ should disappear in $\operatorname{Re} \tilde{O_{x}}(\vec{q})+\operatorname{Re} \tilde{O_{y}}(\vec{q})$ whereas the Bragg-satellite peaks at $\vec{Q}^{\prime}=(1,0) \pm \vec{Q}$ and $\vec{Q}^{\prime \prime}=(0,1) \pm \vec{Q}_{x}$ should exist with opposite sign as shown in figure 4.4 (e) (the same being true for $\operatorname{Im}_{x}(\vec{q})+\operatorname{Im}_{\tilde{O}_{y}}(\vec{q})$ ).

The second predicted characteristic is that the primary CDW peaks at $\pm \vec{Q}$ should exist clearly in $\operatorname{Re} \tilde{O}_{x}(\vec{q})-\operatorname{Re} \tilde{O}_{y}(\vec{q})$ whereas their Bragg-satellite peaks at $\vec{Q}^{\prime}=(1,0) \pm \vec{Q}$ and $\vec{Q}^{\prime \prime}=(0,1) \pm \vec{Q}_{x}$ should disappear (the same being true for $\operatorname{Im} \tilde{O_{x}}(\vec{q})-\operatorname{Im}_{y}(\vec{q})$ ). This is required because, if all $\mathrm{O}_{y}$ sites are multiplied by -1 , as when we take the difference $\operatorname{Re} \tilde{O_{x}}(\vec{q})-\operatorname{Re} \tilde{O_{y}}(\vec{q})$, a $d$-symmetry form factor CDW is converted to an $s$ 'form factor CDW. For this reason, the signature of a $d$-symmetry form factor CDW in $\operatorname{Re} \tilde{O}_{x}(\vec{q})-\operatorname{Re} \tilde{O}_{y}(\vec{q})$ is that it should exhibit the characteristics of figure 4.4 (f). A calculation demonstrating that these signatures are robust, in the presence of arbitrary disorder of the CDW amplitude and phase is presented in appendix C. 

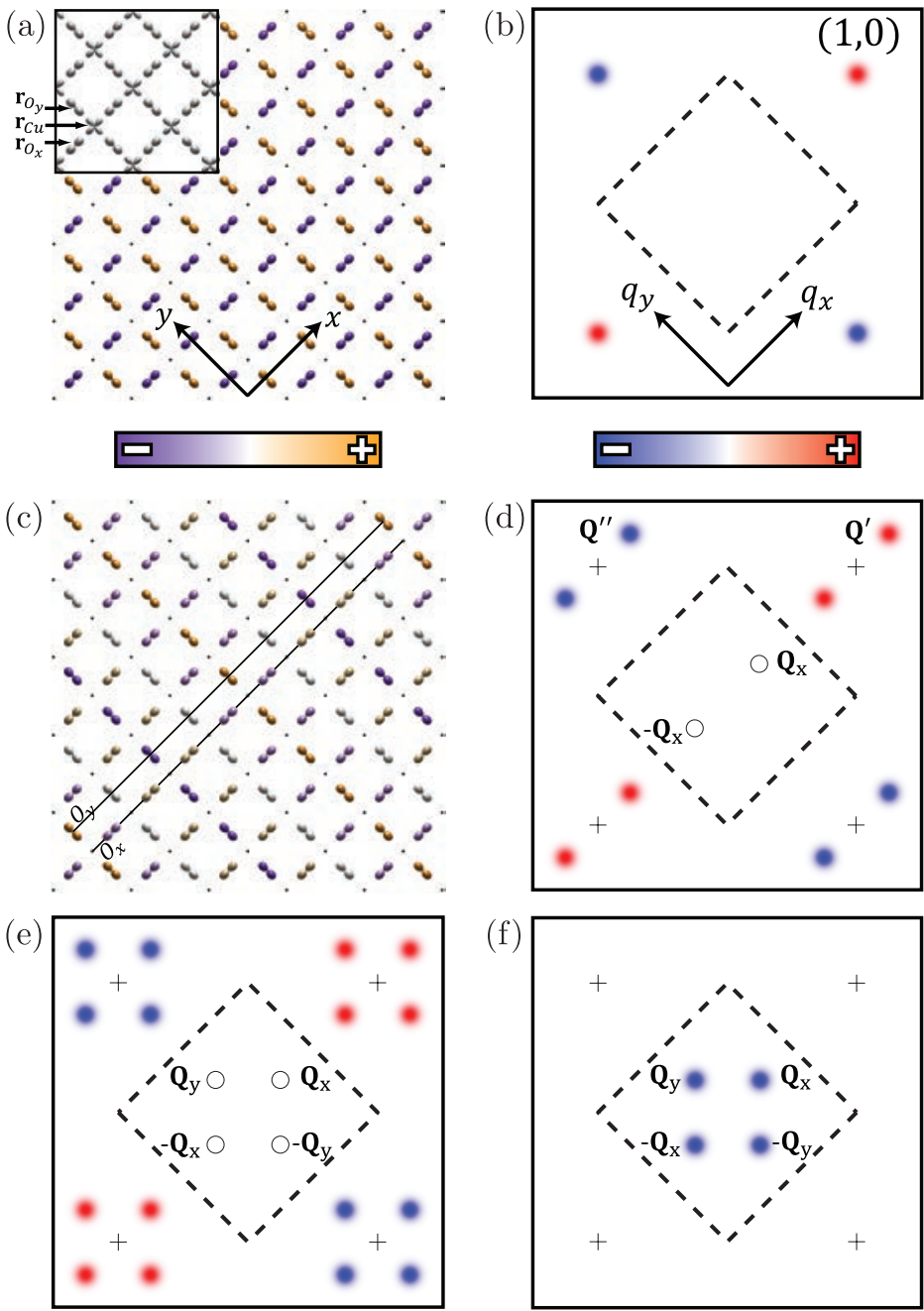

Figure 4.4: (a) Schematic of translationally invariant electronic structure pattern with opposite-sign density on $\mathrm{O}_{x}$ and $\mathrm{O}_{y}$ ( $d$ symmetry). The inactive $\mathrm{Cu}$ sites are indicated by gray circles. Inset shows elementary $\mathrm{Cu}, \mathrm{O}_{x}$, and $\mathrm{O}_{y}$ orbitals, on the three sub-lattices in the $\mathrm{CuO}_{2}$ plane. (b) Fourier transform of the $\vec{q}=0 \mathrm{C}_{4}$-breaking pattern of opposite-sign density on $\mathrm{O}_{x}$ and $\mathrm{O}_{y}$ in (a). The Bragg peaks have the opposite sign, as a result of the intra-unit-cell states having $d$-symmetry. (c) Schematic of a $d$-symmetry form factor CDW $\rho_{D}(\vec{r})=D(\vec{r}) \cos \left(\vec{Q}_{x} \cdot \vec{r}\right)$ with the origin chosen at a $\mathrm{Cu}$ site and modulating only along the x-axis. The colors represent the density $\rho_{D}(\vec{r})$ at every oxygen site. Two thin lines are shown; one labeled $\mathrm{O}_{x}$ traverses parallel to $\vec{Q}_{x}$ and passes only through oxygen sites oriented along the $\mathrm{x}$ axis, and the second one labeled $\mathrm{O}_{y}$ again traverses parallel to $\vec{Q}_{x}$ but passes only through oxygen sites oriented along the y axis. The colour scale demonstrates how the amplitude of the CDW is exactly $\pi$ out of phase along these two trajectories. (d) The real component of the Fourier transform of the pattern in (c). For this $d$-symmetry form factor CDW, the Bragg-satellite peaks at $\vec{Q}^{\prime}$ and $\vec{Q}^{\prime \prime}$ exhibit opposite sign. More profoundly, because they are out of phase by $\pi$, the modulations of $\mathrm{O}_{x}$ and $\mathrm{O}_{y}$ sites cancel, resulting in the disappearance of the CDW modulation peaks at $\pm \vec{Q}$ within the first Brillouin zone (dashed box). (e) The Fourier transform $\operatorname{Re} \tilde{O_{x}}(\vec{q})+\operatorname{Re} \tilde{O_{y}}(\vec{q})$ of a dsymmetry form factor CDW having modulations at $\vec{Q}=(Q, 0) ;(0, Q)$; the CDW satellites at $\vec{Q}^{\prime}$ and $\vec{Q}^{\prime \prime}$ exhibit opposite sign, and the primary CDW modulation peaks at $\pm \vec{Q}$ have disappeared from within the first Brillouin zone, as indicated by open circles. (f) The Fourier transform $\operatorname{Re} \tilde{O_{x}}(\vec{q})-\operatorname{Re} \tilde{O_{y}}(\vec{q})$ of a dsymmetry form factor CDW having modulations at $\vec{Q}=(Q, 0) ;(0, Q)$; the CDW satellites of inequivalent Bragg peaks at $\vec{Q}^{\prime}$ and $\vec{Q}^{\prime \prime}$ now cancel whereas the primary CDW modulation peaks at $\pm \vec{Q}$ are robust within the first Brillouin zone. 


\subsection{Detection of a $d$-Symmetry Form Factor CDW in Cuprates}

In this section I will present sub-lattice segregated, phase-resolved SI-STM data on the short-ranged incommensurate $\mathrm{CDW}$ in $\mathrm{Bi}_{2} \mathrm{Sr}_{2} \mathrm{CaCu}_{2} \mathrm{O}_{8+\delta}$ and $\mathrm{Na}_{x} \mathrm{Ca}_{2-x} \mathrm{CuO}_{2} \mathrm{Cl}_{2}$ [27, $58,111]$. These data provide a direct determination of the CDW form factor, allowing the hypothesis that these CDWs exhibit a predominantly $d$-symmetry form factor to be tested.

Throughout this section I will refer to the non-dispersing modulations at wave-vectors $\vec{Q}_{x} \approx(0.25,0) 2 \pi / a_{0}$ and $\vec{Q}_{y} \approx(0,0.25) 2 \pi / a_{0}$ observed in SI-STM spectroscopic maps as a CDW. This is justified because, despite STM not directly measuring local charge density, the quantities measured by STM are scalar, phase invariant and time-reversal invariant quantities. This does not in itself mean that the state is not best described microscopically by another order such as a spin density wave or pair density wave but, as described in section 6.3, these orders will generally induce attendant CDW components. The only modulations that may manifest in the local density of states are those of the symmetry allowed CDW components. In this sense, the assignment of non-dispersive modulations in SI-STM spectroscopic maps to CDW modulations is rigorously justified.

I will begin by analysing the energy integrated function $R(\vec{r}, E)=I(\vec{r},+|E|) / I(\vec{r},-|E|)$ which, as detailed in section 2.4.2, naturally includes greater contributions from the non-dispersing modulations expected of a CDW. It also removes the setup-effect artifact discussed in chapter 2. In section 4.5 I will go on to discuss how the CDW imparts itself on the energy-resolved tunnelling spectra using the functions $g(\vec{r}, E)$ and $Z(\vec{r}, E)=$ $g(\vec{r},+|E|) / g(\vec{r},-|E|)$.

\subsubsection{Form Factor Measurement using Sub-Lattice Segregated SI-STM}

As detailed in chapter 2, to study non-trivial intra-unit-cell structure it is highly advantageous to collect data with many pixels within each unit cell, whilst maintaining a large field-of-view (FOV). This allows for effective discrimination of distinct atomic sites in the lattice. Data acquired under these circumstances are displayed in figure 4.5 (a) which shows the measured $R\left(\vec{r},|E|=150 \mathrm{meV}\right.$ ) for a $\mathrm{Bi}_{2} \mathrm{Sr}_{2} \mathrm{CaCu}_{2} \mathrm{O}_{8+\delta}$ sample 
with hole concentration $p \sim 8 \pm 1 \%$. This field-of-view (FOV) contains $\sim 15,000$ each of individually resolved $\mathrm{Cu}, \mathrm{O}_{x}$, and $\mathrm{O}_{y}$ sites. Figure 4.5 (b) shows a magnified part of this $R(\vec{r})$ with $\mathrm{Cu}$ sites indicated by blue dots.

Figure 4.5 (c) is the simultaneous topographic image from which the positions of the $\mathrm{Cu}, \mathrm{O}_{x}$, and $\mathrm{O}_{y}$ atoms are determined. The atomic contrast in this topograph comes primarily from the $\mathrm{Bi}$ atoms of the $\mathrm{BiO}$ surface layer. These atoms sit directly above the $\mathrm{Cu}$ sites in the $\mathrm{CuO}$ plane and indicate their positions [89]. Oxygen sites are assumed to lie equidistant between $\mathrm{Cu}$ sites. The detailed algorithm for determining atomic positions is discussed in chapter 3 .

Figures $4.5(\mathrm{~d})$ and (e) show the segregation of measured $R(\vec{r})$ into two oxygen sublattice images $O_{x}(\vec{r})$ and $O_{y}(\vec{r})$ determined from figure 4.5 (b). Larger FOV $\mathrm{Cu}, \mathrm{O}_{x}$ and $\mathrm{O}_{y}$ sub-lattice segregated images, derived from figure 4.5 (a), are shown in figure 4.6.

Now consider the complex Fourier transforms of $O_{x}(\vec{r})$ and $O_{y}(\vec{r}), \tilde{O}_{x}(\vec{q})$ and $\tilde{O}_{y}(\vec{q})$, as shown in figures 4.7 (a) and (b). I show only the real part here but both real and imaginary parts are shown in appendix D. Each of these exhibit broad peaks at wavevectors $\vec{Q}_{x, y}$ within the first Brillouin zone. They also exhibit Bragg-satellite peaks at wave-vectors $\vec{Q}^{\prime}=(1,0) 2 \pi / a_{0} \pm \vec{Q}_{x, y}$ and $\vec{Q}^{\prime \prime}=(0,1) 2 \pi / a_{0} \pm \vec{Q}_{x, y}$. In section 4.3 we concluded that to search for a CDW with a predominantly $d$-symmetry form factor we should look at the combinations of these sub-lattice Fourier transforms $\tilde{O}_{x}(\vec{q}) \pm \tilde{O}_{y}(\vec{q})$. Upon calculating the sum $\operatorname{Re} \tilde{O}_{x}(\vec{q})+\operatorname{Re} \tilde{O}_{y}(\vec{q})$ as shown in figure 4.7 (c), we find no CDW modulation peaks in the vicinity of $\vec{Q}_{x, y}$. This is exactly as expected for a CDW with a $d$-symmetry form factor and results from the $\pi$ phase shift between the modulation on $\mathrm{x}$ and $\mathrm{y}$ directed oxygen sub-lattices.

The peaks at $\vec{Q}_{x, y}$ inside the first Brillouin zone that are weak in figures 4.7 (a) and (b) and absent in figure 4.7 (c) are clearly visible in $\operatorname{Re} \tilde{O}_{x}(\vec{q})-\operatorname{Re} \tilde{O}_{y}(\vec{q})$ as shown in figure 4.7 (d). Hence the absence of this feature in $\operatorname{Re} \tilde{O}_{x}(\vec{q})+\operatorname{Re} \tilde{O}_{y}(\vec{q})$ cannot be ascribed to broadness of the features surrounding $\vec{q}=0$; rather, it is due to a high-fidelity phase cancelation between the modulations on $\mathrm{O}_{x}$ and $\mathrm{O}_{y}$ occurring with $\vec{q} \sim \vec{Q}_{x, y}$.

Finally, the Bragg-satellite peaks at $\vec{Q}^{\prime}$ and $\vec{Q}^{\prime \prime}$ that were clear in $\operatorname{Re} \tilde{O}_{x}(\vec{q})+\operatorname{Re} \tilde{O}_{y}(\vec{q})$ are absent in $\operatorname{Re} \tilde{O}_{x}(\vec{q})-\operatorname{Re} \tilde{O}_{y}(\vec{q})$. Comparison of these observations with predictions for a $d$-symmetry form factor CDW in figure 4.4 demonstrates that the modulations at $\vec{Q}_{x, y}$ 


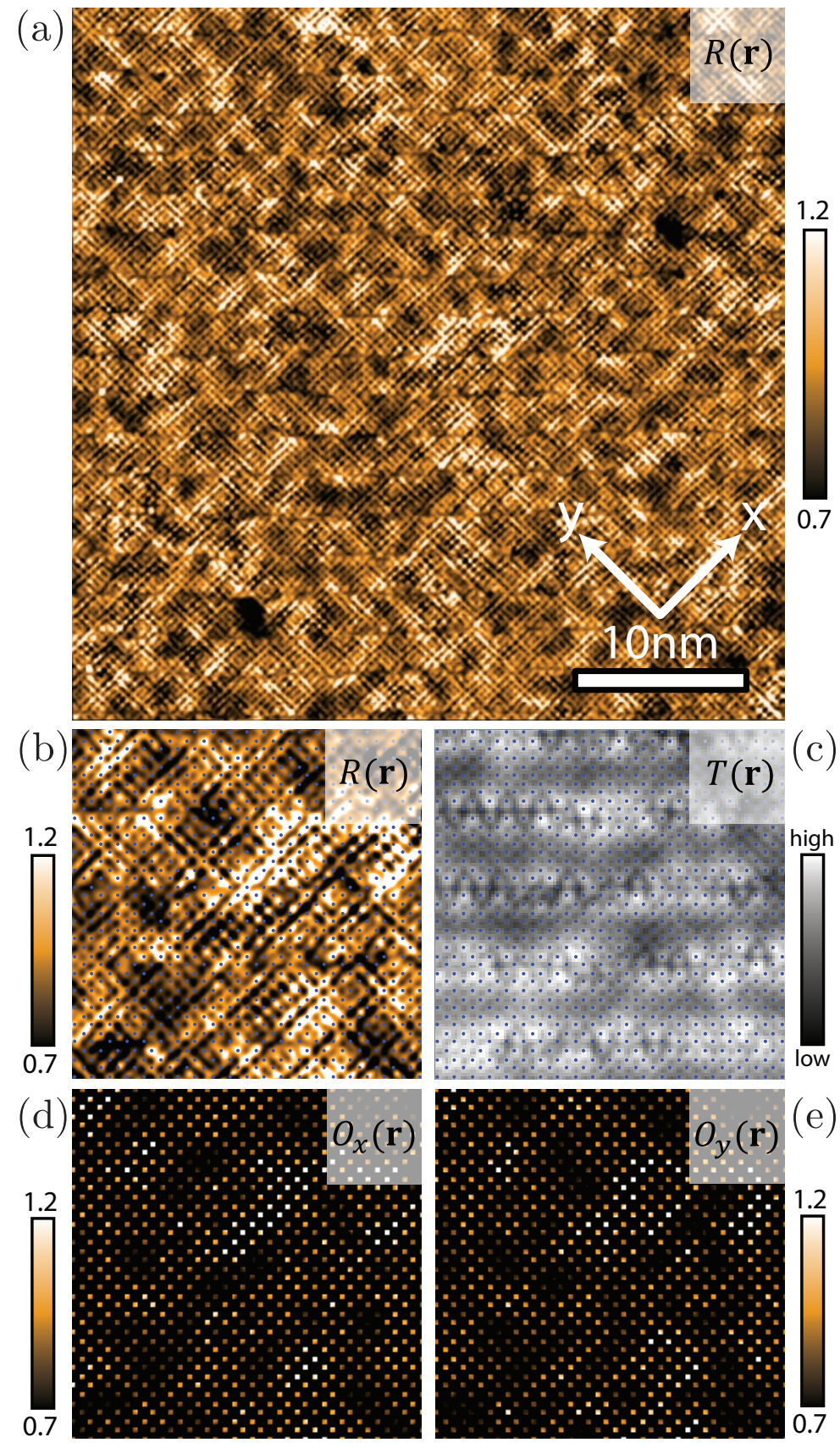

Figure 4.5: (a) Measured $R(\vec{r})$ with $\sim 16$ pixels within each $\mathrm{CuO}_{2}$ unit cell and $\sim 45 \mathrm{~nm}$ square FOV for a $\mathrm{Bi}_{2} \mathrm{Sr}_{2} \mathrm{CaCu}_{2} \mathrm{O}_{8+\delta}$ sample with $p=8 \pm 1 \%$. This $R(\vec{r})$ electronic structure image reveals extensive local intra-unit-cell $\mathrm{C}_{4}$ symmetry breaking $[32,112,113]$. (b) Smaller section of $R(\vec{r})$ in FOV of (a), now showing the location of the $\mathrm{Cu}$ lattice as blue dots. The well-known breaking of $\mathrm{C}_{4}$ rotational symmetry within virtually every $\mathrm{CuO}_{2}$ unit cell and the modulations thereof are obvious. (c) Topographic image of FOV in (b) showing $\mathrm{Cu}$ lattice sites as identified from the $\mathrm{Bi}$ atom locations as blue dots. By using the Lawler-Fujita algorithm [32, 89] spatialphase accuracy for the $\mathrm{CuO}_{2}$ plane of $\sim 0.01 \pi$ is achieved throughout. (d) In the same FOV as (b), we measure the value of $R$ at every $\mathrm{O}_{x}$ site and show the resulting function $O_{x}(\vec{r})$. (e) In the same FOV as (b), we measure the value of $R$ at every $\mathrm{O}_{y}$ site and show the resulting function $O_{y}(\vec{r})$. 

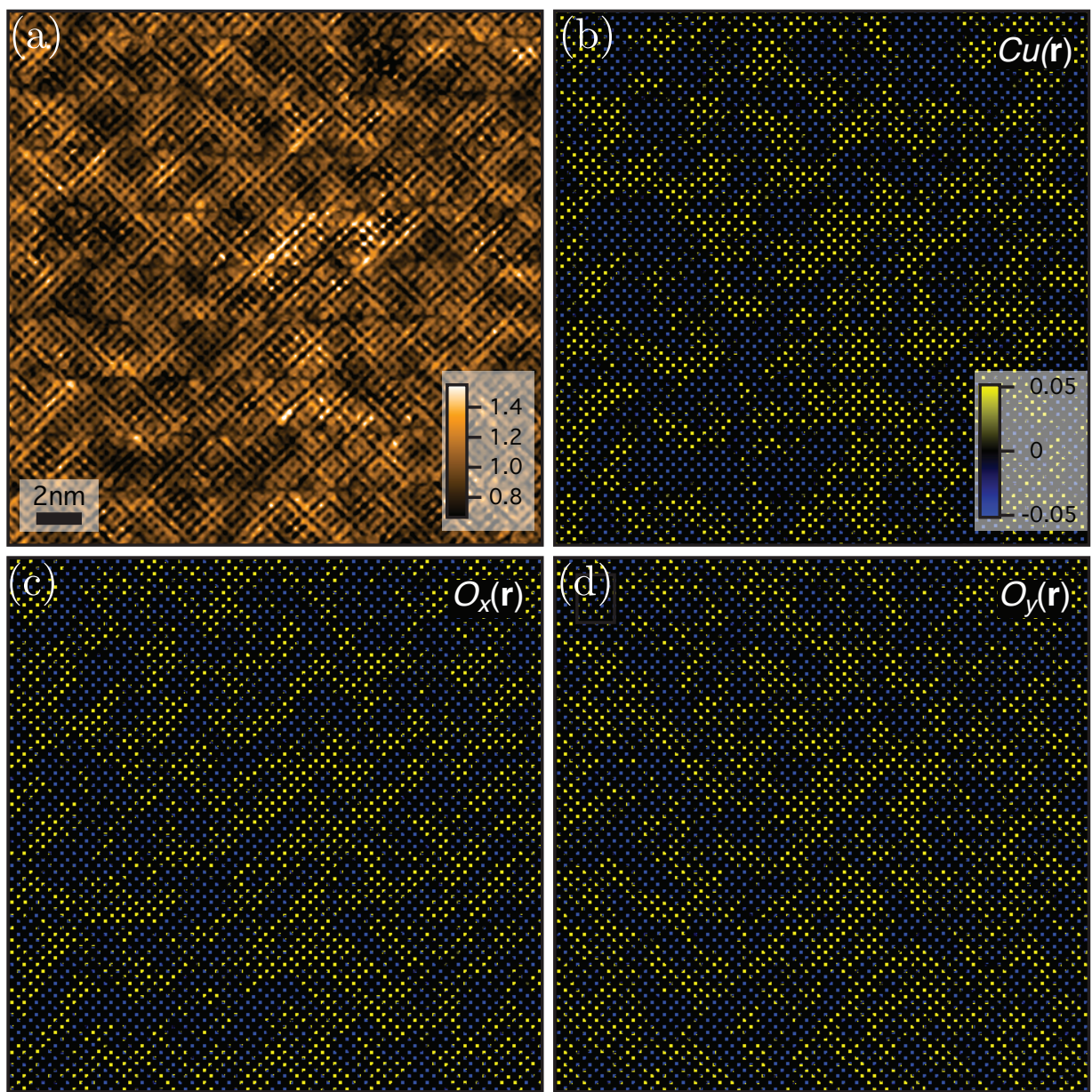

Figure 4.6: (a) Measured $R(\vec{r})$ for $\mathrm{Bi}_{2} \mathrm{Sr}_{2} \mathrm{CaCu}_{2} \mathrm{O}_{8+\delta}$ sample with $p \sim 8 \pm 1 \%$. These data are a subset of figure 4.5 (a). (b) Copper site segregated image, $C u(\vec{r})$, with copper sites selected from (a). (c) x-directed oxygen sub-lattice segregated image, $O_{x}(\vec{r})$, with x-oxygen sites selected from (a). (d) y-directed oxygen-site segregated image, $O_{y}(\vec{r})$, with y-oxygen sites selected from (a). The spatial mean has been subtracted from

$$
\text { (b)-(d). }
$$

maintain a phase difference of approximately $\pi$ between $O_{x}$ and $\mathrm{O}_{y}$ within each unit cell and therefore constitute a CDW with a predominantly $d$-symmetry form factor.

To demonstrate that these phenomena are not a specific property of a given crystal symmetry, surface termination layer, or cuprate material family, we carry out the identical analysis on data from $\mathrm{Na}_{x} \mathrm{Ca}_{2-x} \mathrm{CuO}_{2} \mathrm{Cl}_{2}$ samples with $p \sim 12 \pm 1 \%$. For this compound, figures 4.7 (e) and (f) show the measured $\operatorname{Re} \tilde{O}_{x}(\vec{q})$ and $\operatorname{Re} \tilde{O}_{y}(\vec{q})$. Again, the absence of CDW peaks at $\vec{Q}_{x, y}$ in figure $4.7(\mathrm{~g})$, which shows $\operatorname{Re} \tilde{O}_{x}(\vec{q})+\operatorname{Re} \tilde{O}_{y}(\vec{q})$, is due to cancelation between $\mathrm{O}_{x}$ and $\mathrm{O}_{y}$ contributions, as these peaks are visible in $\operatorname{Re} \tilde{O}_{x}(\vec{q})-\operatorname{Re} \tilde{O}_{y}(\vec{q})$. 


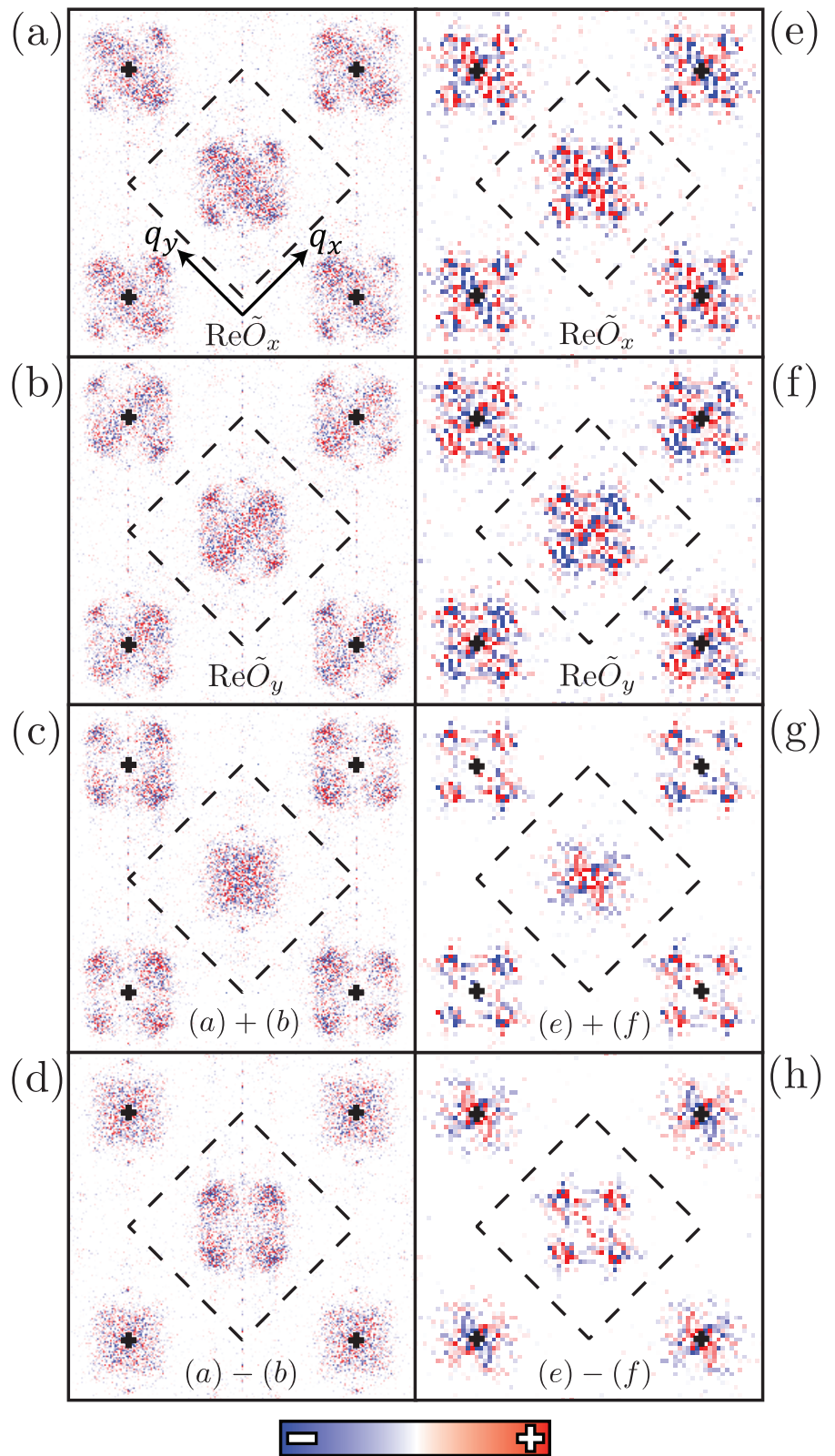

Figure 4.7: (a) Measured $\operatorname{Re} \tilde{O}_{x}$ from $R(\vec{r})$ in figure 4.5 (a). It shows four CDW peaks at $\vec{Q}_{x, y}$ and $16 \mathrm{CDW}$ Bragg-satellite peaks. (b) $R e \tilde{O}_{x}$ from $R(\vec{r})$ in figure 4.5 (a). Again, it shows four CDW peaks at $\vec{Q}_{x, y}$ and $16 \mathrm{CDW}$ Bragg-satellite peaks. (c) Measured $\operatorname{Re} \tilde{O}_{x}(\vec{q})+\operatorname{Re} \tilde{O}_{y}(\vec{q})$ from (a) and (b). The four CDW peaks at $\vec{Q}_{x, y}$ are not detectable whereas the CDW Bragg-satellite peaks are enhanced and well resolved. Compared to figure 4.4, these are the expected phenomena of a $d$-symmetry form factor CDW. (d) Measured $\operatorname{Re} \tilde{O}_{x}(\vec{q})-\operatorname{Re} \tilde{O}_{y}(\vec{q})$ from (a) and (b). The primary CDW peaks at $\vec{Q}_{x, y}$ are strongly enhanced whereas the CDW Bragg-satellite peaks have disappeared. Compared to figure 4.4, these are once again the expected phenomena of a $d$-symmetry form factor. (e) Measured $\operatorname{Re} \tilde{O}_{x}$ for an $\mathrm{Na}_{x} \mathrm{Ca}_{2-x} \mathrm{CuO}_{2} \mathrm{Cl}_{2}$ sample with $p \sim 12 \pm 1 \%$. It shows four CDW peaks at $\vec{Q}_{x, y}$ and $16 \mathrm{CDW}$ Bragg-satellite peaks. (f) Measured $R e \tilde{O}_{x}$ for $\mathrm{Na}_{x} \mathrm{Ca}_{2-x} \mathrm{CuO}_{2} \mathrm{Cl}_{2}$. It also shows four CDW peaks at $\vec{Q}_{x, y}$ and $16 \mathrm{CDW}$ Bragg-satellite peaks. (g) Measured $\operatorname{Re} \tilde{O}_{x}(\vec{q})+\operatorname{Re} \tilde{O}_{y}(\vec{q})$ from (e) and (f). The four CDW peaks at $Q_{x, y}$ are no longer detectable whereas the CDW Bragg-satellite peaks are enhanced and well resolved. Compared to figure 4.4, these are the expected phenomena of a $d$-symmetry form factor CDW. (h) Measured $\operatorname{Re} \tilde{O}_{x}(\vec{q})-\operatorname{Re} \tilde{O}_{y}(\vec{q})$ from (e) and (f). The four CDW peaks at $\vec{Q}_{x, y}$ are enhanced whereas the CDW Bragg-satellite peaks have disappeared. Compared to figure 4.4, these confirm the conclusion that the CDW has a predominantly $d$-symmetry form factor. 
$\operatorname{Re} \tilde{O}_{x}(\vec{q})-\operatorname{Re} \tilde{O}_{y}(\vec{q})$ reveals again that the peaks at $\vec{Q}_{x, y}$ inside the first Brillouin zone that are invisible in figure 4.7 (g) become vivid in figure 4.7 (h), whereas the Bragg-satellites disappear. One can see directly that these results are comprehensively consistent with observations in figures 4.7 (a)-(d), meaning that the CDW of $\mathrm{Na}_{x} \mathrm{Ca}_{2-x} \mathrm{CuO}_{2} \mathrm{Cl}_{2}$ also exhibits a robust $d$-symmetry form factor. This observation rules out materials systematics as the source of the signal and therefore signifies that these $d$-symmetry form factor charge correlations are an intrinsic property of the underdoped $\mathrm{CuO}_{2}$ plane, at least in $\mathrm{Bi}_{2} \mathrm{Sr}_{2} \mathrm{CaCu}_{2} \mathrm{O}_{8+\delta}$ and $\mathrm{Na}_{x} \mathrm{Ca}_{2-x} \mathrm{CuO}_{2} \mathrm{Cl}_{2}$.

One can quantify the magnitude of the $s^{-}, s^{\prime}-$, and $d$-symmetry form factor components of the observed CDW near $\vec{Q}_{x, y}$. As form factor specific measures of CDW amplitude I introduce the complex Fourier transforms

$$
\begin{gathered}
S(\vec{q})=\tilde{C u}(\vec{q}) \\
S^{\prime}(\vec{q})=\frac{1}{2}\left[\tilde{O}_{x}(\vec{q})-\tilde{O}_{y}(\vec{q})\right] \\
D(\vec{q})=\frac{1}{2}\left[\tilde{O}_{x}(\vec{q})-\tilde{O}_{y}(\vec{q})\right],
\end{gathered}
$$

where the factors of two arise because there are twice as many atomic sites included in $S^{\prime}(\vec{r})$ and $D(\vec{r})$ as in $S(\vec{r})$. In figures 4.8 (a)-(c) I show the power spectral densities $|S(\vec{q})|^{2},\left|S^{\prime}(\vec{q})\right|^{2}$ and $|D(\vec{q})|^{2}$. Note that (b) and (c) show the same pattern of peaks as figures 4.7 (c) and (d), indicating that the conclusions drawn from the real part of the Fourier transform hold for the full complex Fourier transform. In figure 4.8 (d), line-cuts along the dashed lines through $\vec{Q}_{x, y}$ in figure 4.8 show that the $d$-form factor component is by far the predominant component. No peak above the background is evident at $\vec{Q}_{x, y}$ in $|S(\vec{q})|^{2}$ and $\left|S^{\prime}(\vec{q})\right|^{2}$.

This information can now be used to understand the real-space patterns observed by intra-unit-cell resolution STM $[32,112,113]$. In figure 4.9 (a) I show one typical wavelength of the CDW in underdoped $\mathrm{Bi}_{2} \mathrm{Sr}_{2} \mathrm{CaCu}_{2} \mathrm{O}_{8+\delta}$ and $\mathrm{Na}_{x} \mathrm{Ca}_{2-x} \mathrm{CuO}_{2} \mathrm{Cl}_{2}$. Here the CDW modulations are almost entirely along the $x$-direction and the electronic structure retains lattice translational symmetry along $y$. I will term this a unidirectional domain.

In figure 4.9 (b) I plot a simulation of one $4 \mathrm{a}_{0}$ wavelength of a unidirectional $d$-symmetry form factor CDW. Comparison with figure 4.9 (a) is a compelling real-space confirmation that the $\mathrm{CDW}$ in $\mathrm{Bi}_{2} \mathrm{Sr}_{2} \mathrm{CaCu}_{2} \mathrm{O}_{8+\delta}$ and $\mathrm{Na}_{x} \mathrm{Ca}_{2-x} \mathrm{CuO}_{2} \mathrm{Cl}_{2}$ is an $\approx 4 \mathrm{a}_{0}$ period 
(a)

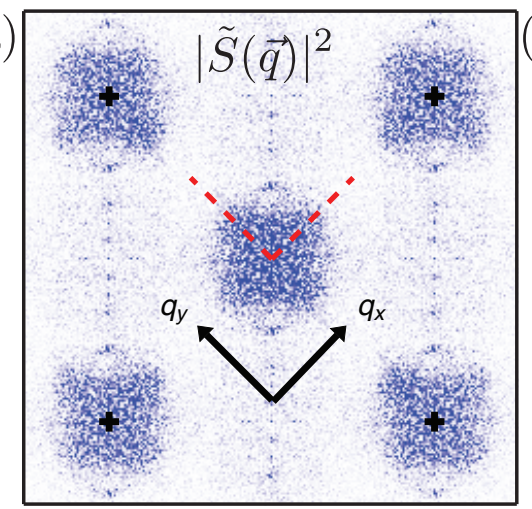

(c)

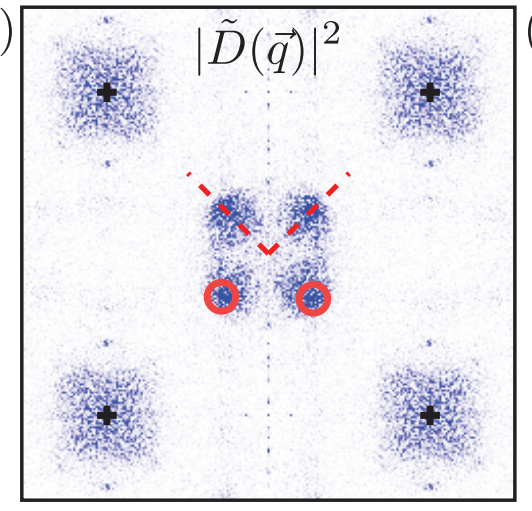

(b)

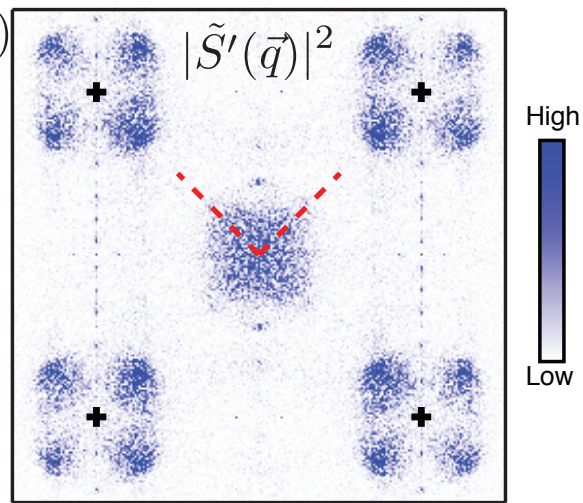

(d)

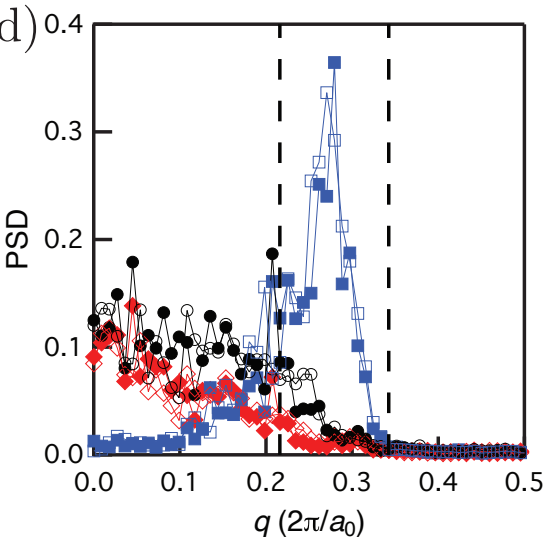

Figure 4.8: Power spectral-density (PSD) Fourier transforms (a) $|S(\vec{q})|^{2}$, (b) $\left|S^{\prime}(\vec{q})\right|^{2}$ and (c) $|D(\vec{q})|^{2}$. The spectral weight proximal to $\vec{Q}_{x, y}$ in these images provides a measure of the relative weight of the $s_{-}, s^{\prime}-$, and $d$-symmetry form factor contributions to the CDW respectively. (d) $|S(\vec{q})|^{2}$ (black), $\left|S^{\prime}(\vec{q})\right|^{2}$ (red) and $|D(\vec{q})|^{2}$ (blue) plotted along the dashed line through $\vec{Q}_{x, y}$ shown in (a)-(c). The $d$-symmetry form factor is clearly predominant.

modulation with a predominately $d$-symmetry form factor.

Recent advances in resonant x-ray scattering methods have allowed access to similar information in the bulk [58]. A special geometry where the sample is rotated by an angle $\alpha$ about an axis parallel to the CDW ordering wave-vector $\vec{Q}$ is used. Through analysis of the scattering intensity for scans in momentum space through $\vec{Q}$ as a function of $\alpha$ and of incident photon polarisation these studies concluded that the charge order in $\mathrm{YBa}_{2} \mathrm{Cu}_{3} \mathrm{O}_{6+x}$ has a predominantly $d$-symmetry form factor [114].

Resonant x-ray scattering is primarily sensitive to modulations in the valence electronic states [58]. In contrast, conventional (non-resonant) x-ray diffraction experiments are sensitive to modulations in the total electronic charge, typically dominated by the concomitant displacement of atomic positions and core electrons. By studying the CDW 

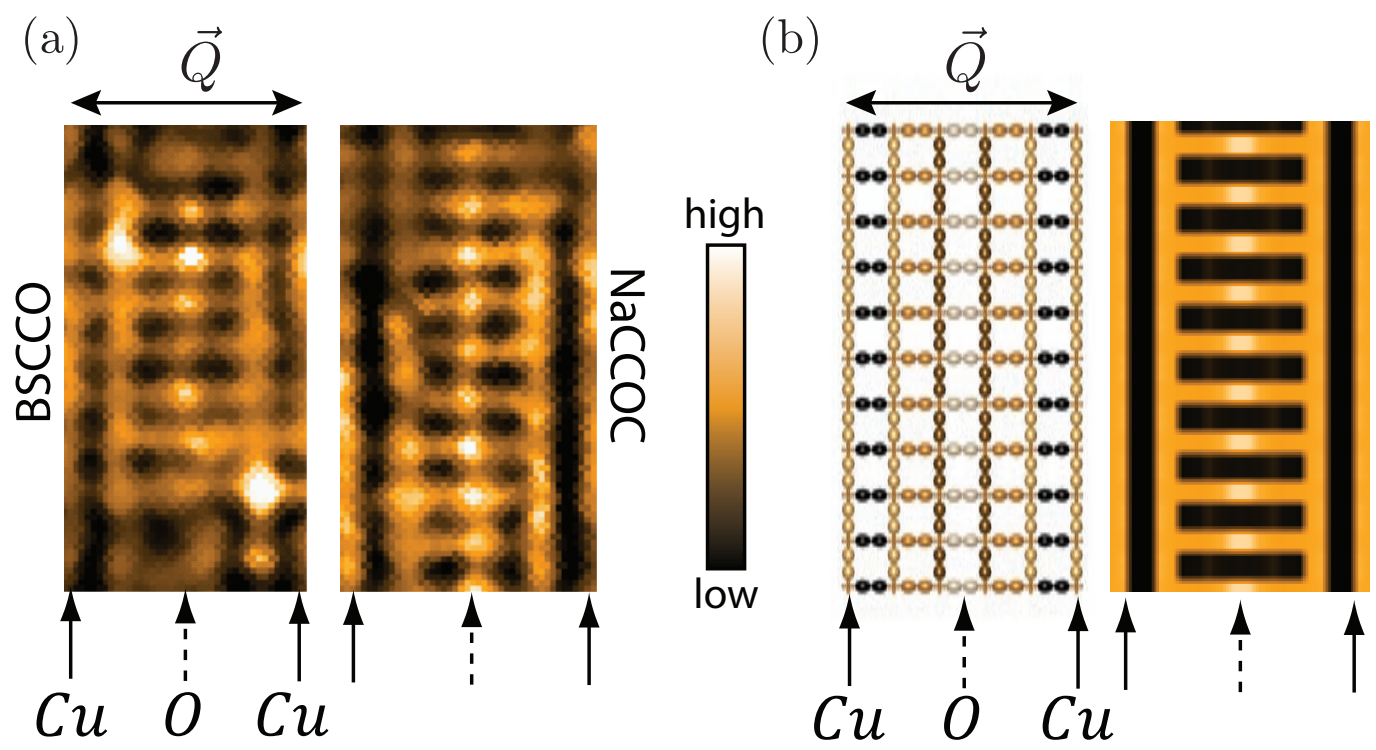

Figure 4.9: (a) Measured $R(\vec{r})$ images of local unidirectional CDW patterns that commonly occur in $\mathrm{Bi}_{2} \mathrm{Sr}_{2} \mathrm{CaCu}_{2} \mathrm{O}_{8+\delta}$ and $\mathrm{Na}_{x} \mathrm{Ca}_{2-x} \mathrm{CuO}_{2} \mathrm{Cl}_{2}$ [32, 112, 113]. The $\mathrm{Cu}$ and $\mathrm{O}_{x}$ sites (labeled by solid and dashed arrows, respectively) were determined independently and directly from topographic images. (b) (Left) $d$-symmetry form factor CDW model with $\vec{Q}=(0.25,0) 2 \pi / a_{0}$ and amplitude maximum on the central $\mathrm{O}_{x}$ site (dashed arrow). (Right) The calculated density-wave pattern from this model. Therefore, a $d$-symmetry form factor CDW model with this particular spatial phase provides an excellent explanation for the patterns observed in (a) and reported previously in [32, $112,113]$.

hard x-ray diffraction peaks in many Brillouin zones for $\mathrm{YBa}_{2} \mathrm{Cu}_{3} \mathrm{O}_{6+x}$, Forgan et al. found the three-dimensional pattern of atomic displacements associated with the CDW. These results are consistent with a predominantly $d$-symmetry form factor [115].

As a counterpoint this, resonant x-ray scattering studies of $\mathrm{La}_{2-x} \mathrm{Ba}_{x} \mathrm{CuO}_{4+\delta}$ have revealed that the charge order has a predominantly $s$ 'symmetry form factor [116]. This is just one of the many differences between charge order in $\mathrm{La}_{2} \mathrm{CuO}_{4}$ based cuprates and the other cuprate materials explored in this chapter.

It is perhaps not surprising that the predominance of one specific form factor of the CDW is not universal across cuprate materials. After all, these form factors do not uniquely determine the point group symmetry of the CDW. Hence, long-range ordered versions of the CDW present in different materials would not constitute different states of matter (based on the set of symmetries they break). However, differences in predominant form factor between materials may give some insight into the microscopic mechanism of charge ordering in these materials. 
The high-temperature superconductivity and the CDW form factor share a $d_{x^{2}-y^{2}}$ symmetry in $\mathrm{Bi}_{2} \mathrm{Sr}_{2} \mathrm{CaCu}_{2} \mathrm{O}_{8+\delta}, \mathrm{Na}_{x} \mathrm{Ca}_{2-x} \mathrm{CuO}_{2} \mathrm{Cl}_{2}$ and $\mathrm{YBa}_{2} \mathrm{Cu}_{3} \mathrm{O}_{6+x}$. Does this observation have any significance? For a certain class of cuprate CDW theories; yes. In spin-fermion "hot-spot" models, CDW and $d$-wave superconductivity are formed by the exchange of $(\pi, \pi)$ spin-flucuations between the eight "hot-spots" where the Fermi surface intersects the anti-ferromagnetic zone boundary [117]. In this situation, there is a deep connection between the symmetry of the superconductivity and the form factor of the CDW because they arise from the same fermions and the same interactions. However, the applicability of these models to real cuprate materials has been questioned [110].

In a less model specific way, $d$-wave superconductivity and short-ranged $d$-symmetry form factor CDW are, in a sense, natural partners. The form factor for scattering of Bogoliubov quasi-particles from a puddle of $d$-symmetry form factor CDW is vanishing at the $d$-wave nodes, thus protecting the coherence of nodal quasi-particles in what have been termed "cold-spots" [118]. It is tempting to draw a connection between the $s$ 'symmetry form factor (for which no such protection exists) and the extremely small $T_{c}$ in the stripe phase of in $\mathrm{La}_{2-x} \mathrm{Ba}_{x} \mathrm{CuO}_{4+\delta}$.

Combining these x-ray studies with our STM results we can conclude that the charge order in $\mathrm{Bi}_{2} \mathrm{Sr}_{2} \mathrm{CaCu}_{2} \mathrm{O}_{8+\delta}, \mathrm{Na}_{x} \mathrm{Ca}_{2-x} \mathrm{CuO}_{2} \mathrm{Cl}_{2}$ and $\mathrm{YBa}_{2} \mathrm{Cu}_{3} \mathrm{O}_{6+x}$ all have a predominantly $d$-symmetry form factor. This discovery is in accord with many theoretical treatments of charge order in the $\mathrm{CuO}_{2}$ plane [90-106]. However, this does not in itself allow us to discriminate between different mechanism of CDW formation. For this we will require additional information. To this end, the following sections will investigate the temperature dependence of the CDW, whether it is unidirectional (stripe-like) or bidirectional (chequerboard-like) and how the CDW manifests itself in the energy resolved tunnelling spectrum.

\subsubsection{Temperature Dependence of the CDW Phenomena}

So far all data shown were taken deep in the superconducting state $\left(T \ll T_{c}\right)$. However, x-ray scattering studies show that the CDW correlations onset at a temperature $T_{\mathrm{CDW}}>$ $T_{c}$ [58]. I will now compare and contrast sub-lattice segregated SI-STM data taken with $T<T_{\mathrm{CDW}}$ but for $T<T_{c}$ and $T>T_{c}$. Figure 4.10 (a) shows a typical $Z(\vec{r}, 150 \mathrm{meV})$ 
image of $\mathrm{Bi}_{2} \mathrm{Sr}_{2} \mathrm{CaCu}_{2} \mathrm{O}_{8+x}$ with $p=8 \%$ for $T<T_{c}$ in the superconducting phase. Figure 4.10 (b) shows the equivalent $Z(\vec{r}, 150 \mathrm{meV})$ for $T>T_{c}$ in the cuprate pseudogap phase for a sample of the same doping. Both visually in real-space and through their Fourier transforms $|\tilde{D}(\vec{q})|^{2}$, no pronounced differences in phenomenology are observed between the two.

The clear peaks at $\vec{Q}_{x, y}$ in $|\tilde{D}(\vec{q})|^{2}$ (figures 4.10 (c) and (d)) with approximately the same line-width indicate a predominantly $d$-symmetry form factor CDW with approximately the same correlation length above and below $T_{c}$. Figure 4.10 shows some evidence for an enhanced $s^{\prime}$ component of the CDW above $T_{c}$, but this has not been systematically studied.

These observations show that the CDW detected in $\mathrm{Bi}_{2} \mathrm{Sr}_{2} \mathrm{CaCu}_{2} \mathrm{O}_{8+\delta}$ for $T \ll T_{c}$ persists to temperatures above $T_{c}$ (but below $T_{\mathrm{CDW}}$ ) with little change in phenomenology. That these conductance modulations (specifically those at $|E| \approx \Delta_{1}$ ) are essentially unchanged between $T<T_{c}$ and $T>T_{c}$ is significant for their interpretation. It implies that these modulations are not the spectroscopic signature of a modulation in charge caused by the co-existence of the $d$-wave superconductivity with a pair density wave with wave-vector $\vec{Q}_{x, y}$. As will be explained in detail in section 6.6, this scenario would entail a disappearance of the conductance modulations above $T_{c}$ which is clearly not observed in experiment.

\subsubsection{Short-Range Unidirectional CDW Domains}

I will now go on to discuss how the objects shown in figure 4.9 (a) are tiled in space to fill a large field of view. Although elements indistinguishable from figure 4.9 (a) can be seen in figures 4.10 (a) and (b), no long-range order is obvious. In Fourier space this manifests itself as the broad peaks in figures 4.10 (c) and (d). These correspond to a spatial correlation length of $\approx 24 \AA\left(6.3 a_{0}\right.$ or $\sim 1.6 \mathrm{CDW}$ wavelengths).

To explore the spatial arrangements of the CDW I introduce the complex functions $D_{x}(\vec{r})$ and $D_{y}(\vec{r})$, which are a spatially-resolved measure of the $d$-symmetry form factor amplitude. $D(\vec{q})$ is Fourier filtered around the wave-vectors $\vec{Q}_{x, y}$ to determine the complex-valued $D_{x, y}(\vec{r})$, as detailed in section 2.8. Summarising, we take take the full complex Fourier transform $D(\vec{q})$ and Fourier filter to create two complex real-space maps 

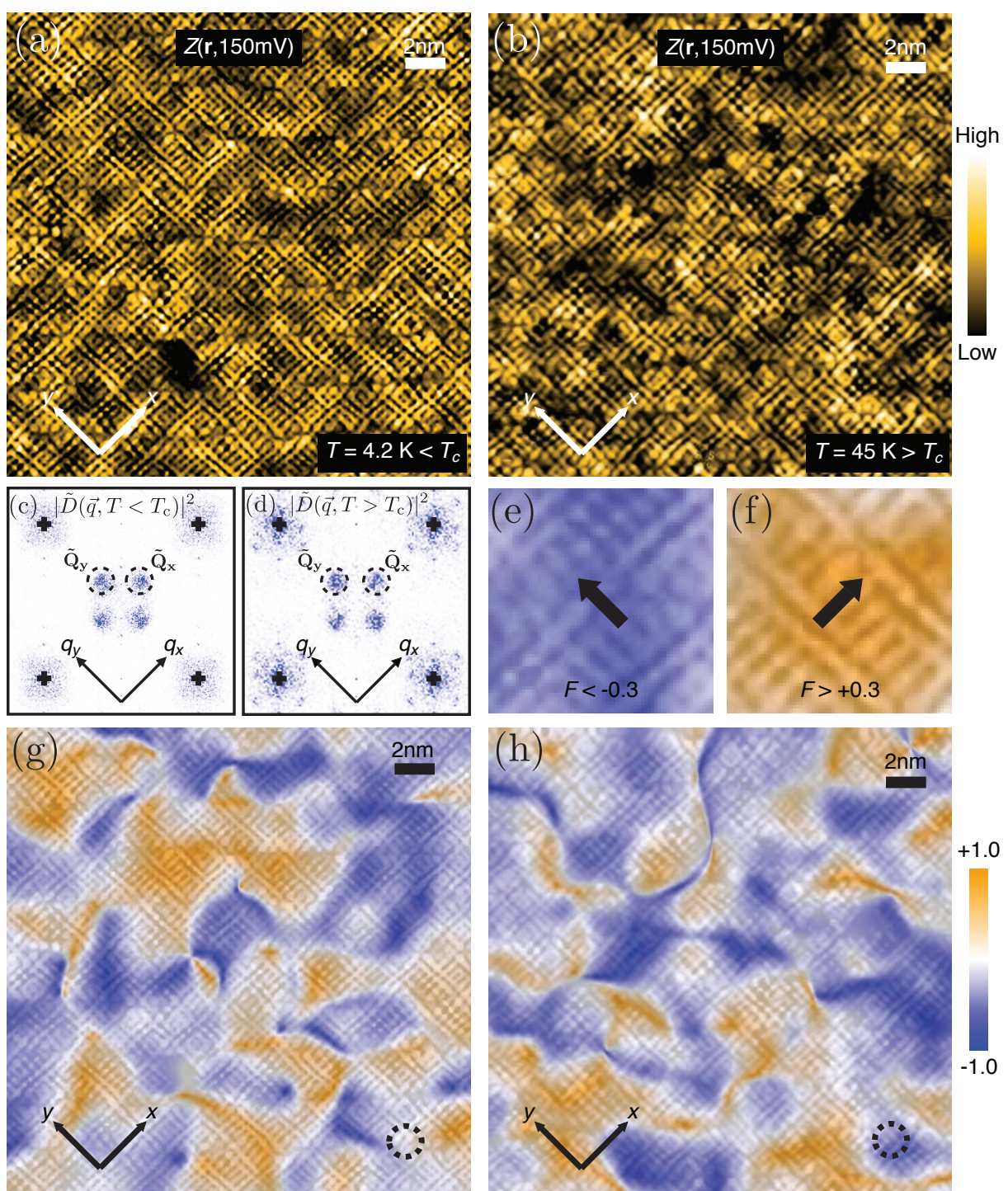

Figure 4.10: (a) Measured $Z(\vec{r}, 150 \mathrm{meV})$ at $T<T_{c}$ in the superconducting phase of $p \sim 8 \%$ hole-doped $\mathrm{Bi}_{2} \mathrm{Sr}_{2} \mathrm{CaCu}_{2} \mathrm{O}_{8+\delta}(T=4.2 \mathrm{~K})$. (b) Measured $Z(\vec{r}, 150 \mathrm{meV})$ at $T>T_{c}$ in the pseudogap phase of $p \sim 8 \%$ hole-doped $\mathrm{Bi}_{2} \mathrm{Sr}_{2} \mathrm{CaCu}_{2} \mathrm{O}_{8+\delta}(T \sim 45 \mathrm{~K})$. The CDW phenomena are essentially indistinguishable from observations at $T<T_{c}$. (c) The $d$-symmetry form factor power spectral density $\left|D^{Z}(\vec{q},|E|)\right|^{2}=\mid\left(\tilde{O}_{x}^{Z}(\vec{q},|E|)-\right.$ $\left.\tilde{O}_{y}^{Z}(\vec{q},|E|)\right) /\left.2\right|^{2}$ determined from sub-lattice segregated analysis of data in (a). (d) $\left|D^{Z}(\vec{q},|E|)\right|^{2}$ determined from sub-lattice segregated analysis of data in (b). The $\vec{q}-$ space structure of the CDW phenomenology is identical in the pseudogap phase and in the superconducting phase (apart from a slightly larger $s$-symmetry form factor component). (e,f) Using only the regions within the dashed circles in (c) and (d) the $\vec{r}$-space amplitudes of the CDW in (a) and (b) are calculated for modulations along $\vec{Q}_{x}$ and $\vec{Q}_{y}$. Then using $F(\vec{r})=\left(\left|D_{x}(\vec{r})\right|-\left|D_{y}(\vec{r})\right|\right) /\left(\left|D_{x}(\vec{r})+\right| D_{y}(\vec{r}) \mid\right)$ regions primarily modulating along y-axis with $-1.0<F(\vec{r})<-0.3$ are shaded blue. Regions primarily modulating along x-axis with $+0.3<F(\vec{r})<+1.0$ are shaded orange. (g) Configuration of unidirectional $d$-symmertry form factor CDW modulations contained in (a) at $T<T_{c}$. The unidirectionality colour scale for $F(\vec{r})$ demonstrated in (e) and (f) is overlaid on the data in (a). The dashed circle shows the $\vec{r}$-space radius equivalent to the $\vec{q}$-space filter used to generate the $D_{x, y}(\vec{r})$ images by Fourier filtering. (h) Domain configuration of unidirectional $d$-symmetry form factor CDW modulations contained in figure at $T>T_{c}$. The unidirectionality colour scale for $F(\vec{r})$ demonstrated in (e) and

(f) is overlaid on the data in (b). The dashed circle has same definition as in (g). 
of $d$-symmetry Form Factor CDW amplitude at wave-vectors $\vec{Q}_{x, y}$,

$$
\begin{aligned}
& D_{x}(\vec{r})=\frac{2}{(2 \pi)^{2}} \int d \vec{q} e^{i \vec{q} \cdot \vec{r}} \tilde{D}(\vec{q}) e^{-\frac{\left(\vec{q}-\vec{Q}_{x}\right)^{2}}{2 \Lambda^{2}}} \\
& D_{y}(\vec{r})=\frac{2}{(2 \pi)^{2}} \int d \vec{q} e^{i \vec{q} \cdot \vec{r}} \tilde{D}(\vec{q}) e^{-\frac{\left(\vec{q}-\vec{Q}_{y}\right)^{2}}{2 \Lambda^{2}}}
\end{aligned}
$$

where $\Lambda^{-1}$ is the characteristic length scale over which variations in $D_{x, y}(\vec{r})$ can be resolved and is set by the filter width in Fourier space. The magnitudes of these functions,

$$
\begin{aligned}
& \left|D_{x}(\vec{r})\right|=\sqrt{\left.\left(\operatorname{Re} D_{x}(\vec{r})\right)^{2}+\operatorname{Im} D_{x}(\vec{r})\right)^{2}} \\
& \left|D_{y}(\vec{r})\right|=\sqrt{\left.\left(\operatorname{Re} D_{y}(\vec{r})\right)^{2}+\operatorname{Im} D_{y}(\vec{r})\right)^{2}}
\end{aligned}
$$

represent the local amplitude of $d$-symmetry form factor CDW modulations along $\vec{Q}_{x}$ and $\vec{Q}_{y}$ respectively. As such, we may visualise the local directionality of CDW domains through

$$
F(\vec{r})=\frac{\mid D_{x}\left(\vec{r}|-| D_{y}(\vec{r} \mid\right.}{\mid D_{x}\left(\vec{r}|+| D_{y}(\vec{r} \mid\right.} .
$$

Through its sign, this function will identify regions where the CDW modulation is primarily along the $\mathrm{x}$ or $\mathrm{y}$ axis.

Figures 4.10 (e) and (f) shows how regions of $-1.0<F(\vec{r})<-0.3$ (shaded blue) are primarily modulating along y-axis whereas regions $+0.3<F(\vec{r})<+1.0$ (shaded orange) are primarily modulating along x-axis (those with $-0.3<F(\vec{r})<+0.3$ shaded white appear at boundaries). Figures 4.10 (g) and (h) reveal the results of this analysis for the data in figures 4.10 (a) and (b) respectively.

Overall, the system is configured into spatial regions within which the CDW along only one direction is dominant. By overlaying the colour scale for $F(\vec{r})$ on the data in figures 4.10 (a) and (b) to create figures 4.10 (g) and (h), one can see directly these unidirectional domains of short-ranged CDW.

That the CDW in $\mathrm{Bi}_{2} \mathrm{Sr}_{2} \mathrm{CaCu}_{2} \mathrm{O}_{8+\delta}$ is short-ranged (short correlation length) is quantitatively consistent with x-ray scattering studies [119]. Similarly short correlation lengths $(\lesssim 80 \AA)$ are observed in $\mathrm{Bi}_{2-y} \mathrm{~Pb}_{y} \mathrm{Sr}_{2-x} \mathrm{La}_{x} \mathrm{CuO}_{6+\delta}, \mathrm{HgBa}_{2} \mathrm{CuO}_{4+\delta}$ and $\mathrm{YBa}_{2} \mathrm{Cu}_{3} \mathrm{O}_{6+x}$ $[50,58,120,121]$. That the CDW is not long-ranged in these materials is not surprising. A Ginzburg-Landau analysis shows that an incommensurate CDW cannot be truly long ranged ordered in a system with quenched charge disorder, which is present in all of 
these materials [122]. But is disorder the factor limiting correlation lengths or are the short correlation lengths a more intrinsic property of the $\mathrm{CuO}_{2}$ plane?

The question of whether disorder is limiting the zero magnetic field CDW correlation length in $\mathrm{YBa}_{2} \mathrm{Cu}_{3} \mathrm{O}_{6+x}$ was addressed by Achkar et al [123]. They used resonant x-ray scattering to measure oxygen-ordered $\mathrm{YBa}_{2} \mathrm{Cu}_{3} \mathrm{O}_{6+x}$ samples before and after disordering the oxygens in the chain layer by heating and quench-cooling. They concluded that while the additional disorder did reduce the scattering intensity in the CDW peaks, it did not significantly alter the wave-vector or correlation length. This suggests that disorder is not the factor limiting the CDW correlation length in $\mathrm{YBa}_{2} \mathrm{Cu}_{3} \mathrm{O}_{6+x}$.

That the in-plane correlation length is seen to peak and then decrease again below $T_{c}$ with decreasing temperature in $\mathrm{YBa}_{2} \mathrm{Cu}_{3} \mathrm{O}_{6+x}$ suggests the role of superconductivity in limiting the correlation length [124, 125]. Further weight is added to this by the observation in $\mathrm{YBa}_{2} \mathrm{Cu}_{3} \mathrm{O}_{6+x}$, at $\mathrm{B}=30 \mathrm{~T}$, of a magnetic field induced $\mathrm{CDW}$ with a resolution limited in-plane correlation length $\xi \geq 230 \AA$ [56].

In our STM studies of both $\mathrm{Bi}_{2} \mathrm{Sr}_{2} \mathrm{CaCu}_{2} \mathrm{O}_{8+\delta}$ and $\mathrm{Na}_{x} \mathrm{Ca}_{2-x} \mathrm{CuO}_{2} \mathrm{Cl}_{2}$ we find that the short-range ordered CDW takes the form of small locally unidirectional domains. These observations of coexisting nanoscale unidirectional regions are in reasonable agreement with deductions from x-ray studies of $\mathrm{YBa}_{2} \mathrm{Cu}_{3} \mathrm{O}_{6+x}$ [126]. Again, the question of whether this unidirectionality is property of the quenched disorder or instead something intrinsic to the $\mathrm{CuO}_{2}$ plane arises [127].

Interestingly the high field CDW observed in $\mathrm{YBa}_{2} \mathrm{Cu}_{3} \mathrm{O}_{6+x}$ appears to be completely unidirectional $[55,56]$. However, the orthorhombicity of $\mathrm{YBa}_{2} \mathrm{Cu}_{3} \mathrm{O}_{6+x}$ means that this is not a true breaking of rotational symmetry because the $\mathrm{Cu}-\mathrm{O}$ directions were not equivalent to begin with. One could argue, though, that the symmetry breaking field provided by the orthorhombicity is sufficiently weak that the observed unidirectionality reflects an intrinsic property of the $\mathrm{CuO}_{2}$ plane. This is supported by theoretical work finding that unidirectional (striped) states have a lower energy than bi-directional (chequerboard) states [95, 128].

One way to determine whether this unidirectionality is truly an intrinsic property of the $\mathrm{CuO}_{2}$ plane would be to search for a inequivalence of the magnetic field induced CDW at wave-vectors $\vec{Q}_{x}$ and $\vec{Q}_{y}$ in a material whose crystal structure has symmetry 
equivalent $\mathrm{Cu}-\mathrm{O}$ directions. The observation of any inequivalence in the field enhanced $\mathrm{CDW}$ amplitude between the two $\mathrm{Cu}-\mathrm{O}$ directions would be a positive identification of an intrinsic tendency toward unidirectional CDW order in the $\mathrm{CuO}_{2}$ planes.

\subsection{Characteristics of CDW Modulations in the Spectral Function}

Figure 4.11 shows how a typical tunnelling conductance spectrum representative of strongly underdoped cuprates exhibits two characteristic energies. The lower energy scale $\Delta_{0}$ represents the maximum energy at which Bogoliubov quasi-particle interference (QPI) modulations are observed [29, 112] while the higher energy scale $\Delta_{1}$ (dashed blue line) is the cuprate pseudogap energy scale, as determined from its comparison with the doping dependence of the pseudogap in photoemission [27].

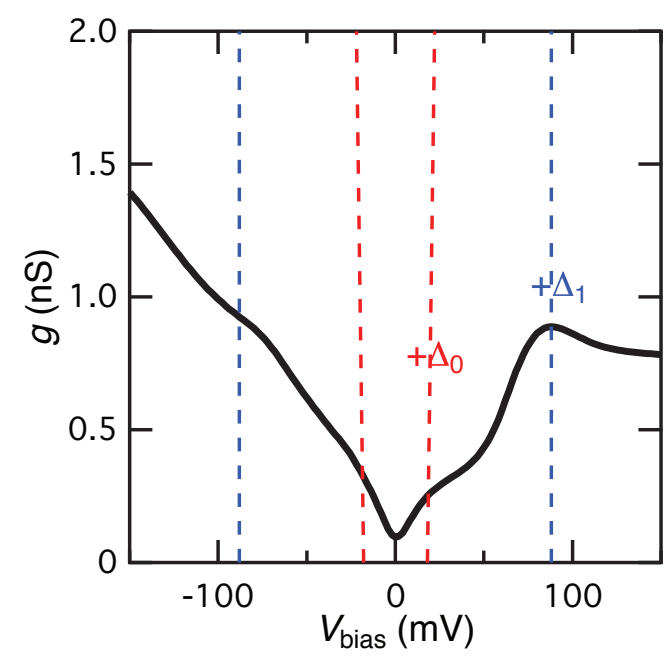

Figure 4.11: The tunnelling differential conductance spectrum $g(E=e V)=d I / d V$ for a $\mathrm{Bi}_{2} \mathrm{Sr}_{2} \mathrm{CaCu}_{2} \mathrm{O}_{8+\delta}$ sample with $p \sim 6 \%$, typical of underdoped cuprates. The energy $\Delta_{0}$ beyond which Bogoliubov QPI is not observed [29, 112] and the pseudogap energy $\Delta_{1}$ are indicated by dashed red and blue lines respectively.

These two energy scales, $\Delta_{0}$ and $\Delta_{1}$, are also manifest in the set of spatial modulations observed in $Z(\vec{r},|E|)$. For $|E|<\Delta_{0}$ a set of dispersive wave-vectors $\vec{q}_{1}, \vec{q}_{2}, \ldots \vec{q}_{7}$ arising from Bogoliubov QPI is observed in $Z(\vec{q},|E|)[27]$. For energies $|E|>\Delta_{0}$ these dispersive wave-vectors give way to ultra-slowly dispersing CDW modulations at $\vec{q}_{1}^{*} \approx \vec{q}_{1}\left(\Delta_{0}\right)$ and $\vec{q}_{5}^{*} \approx \vec{q}_{5}\left(\Delta_{0}\right)$, as shown in figure 4.12 . Importantly, $\vec{q}_{5}^{*}=2 \pi / a_{0}-\vec{q}_{5}^{*}$, signifying that these two wave-vectors correspond to two different form factor components of the same CDW 
modulation. Those at $\vec{q}_{1}^{*}$ correspond to CDW modulations with $s$ - $/ s^{\prime}$ - symmetry form factor whereas those at $\vec{q}_{5}^{*}$ have $d$-symmetry form factor. With increasing energy the $s$ - $/ s$ ' form factor CDW modulations quickly die off and those with $d$-symmetry form factor grow to a maximum at the pseudogap energy scale $|E|=\Delta_{1}[29,112]$.

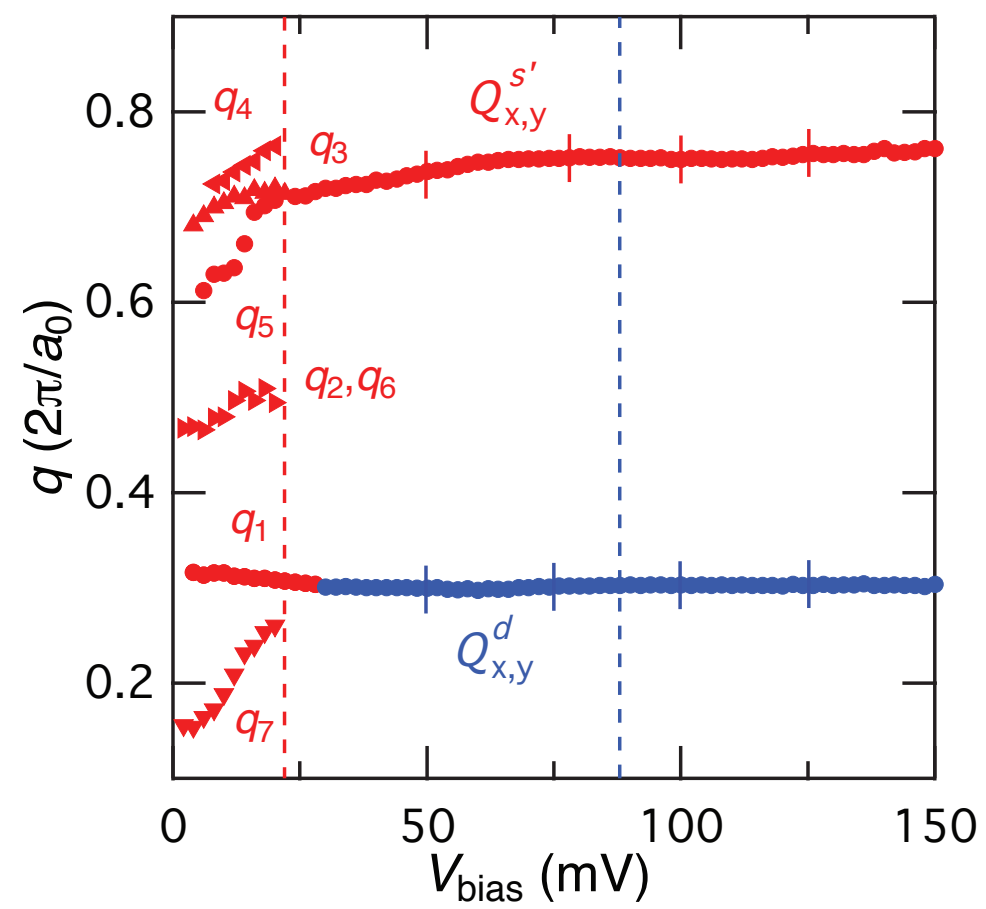

FIGURE 4.12: The energy dispersion of seven dispersive modulations characteristic of Bogoliubov QPI in cuprate superconductors $\left(\vec{q}_{1} \ldots \vec{q}_{7}\right)$ are shown as red triangles, except for $\vec{q}_{1}$ and $\vec{q}_{5}$ which are plotted as red circles. These Bogoliubov QPI modulations are all simultaneously observable only below the energy $\Delta_{0}$, as indicated by dashed red line. Here we demonstrate that they exhibit a predominantly $s$ '-symmetry form factor, indicated by the red colour. At energies above $\Delta_{0}, Z(\vec{r}, E)$ evolves quickly to consist of only non-dispersive $\vec{Q}_{x}$ and $\vec{Q}_{y}$ wave-vectors of the $d$-symmetry form factor CDW. We plot the dispersion of these modulations by showing the energy dependence of the maxima in $\left|\tilde{D}^{Z}(\vec{q}, E)\right|$ as blue squares, with the blue bars representing the full width at half maximum (FWHM) of each such peak. The same physical modulations when analysed using $\left|S^{\prime Z}(\vec{q},|E|)\right|$ appear as the non-dispersive wave-vectors shifted by one reciprocal lattice vector shown as red circles, with the red bars representing the FHWM of each such peak.

In the previous section I showed, through the use of $R(\vec{r})$, that CDW modulations in the tunnelling current, arising from the integrated contributions of states up to an energy $|E|=150 \mathrm{meV}$ away from the chemical potential, exhibit a predominantly $d$ symmetry form factor. In the following I will use the energy resolved functions $g(\vec{r}, E)$ and $Z(\vec{r}, E)=g(\vec{r},+|E|) / g(\vec{r},-|E|)$ to investigate which electronic states, labelled by energy and momentum, are primarily responsible for these modulations. 
To this end I introduce the energy-resolved counterparts to equations 4.8 derived from $Z(\vec{r},|E|)$,

$$
\begin{gathered}
S^{Z}(\vec{q},|E|)=\tilde{C u}^{Z}(\vec{q},|E|) \\
S^{Z}(\vec{q},|E|)=\frac{1}{2}\left[\tilde{O}_{x}^{Z}(\vec{q},|E|)-\tilde{O}_{y}^{Z}(\vec{q},|E|)\right] \\
D^{Z}(\vec{q},|E|)=\frac{1}{2}\left[\tilde{O}_{x}^{Z}(\vec{q},|E|)-\tilde{O}_{y}^{Z}(\vec{q},|E|)\right] .
\end{gathered}
$$

Examples of these form factor specific Fourier transforms are shown in figure 4.13. Peaks proximal to the wave-vectors $\vec{Q}_{x}$ and $\vec{Q}_{y}$ (circled by broken lines) signify CDW modulations with $s^{-}, s^{\prime}$ - and $d$-symmetry form factors respectively. Note that $d$-symmetry form factor modulations appear in $S^{\prime}(\vec{r},|E|)$ and visa versa but shifted by a reciprocal lattice vector as show in figure 4.14 .

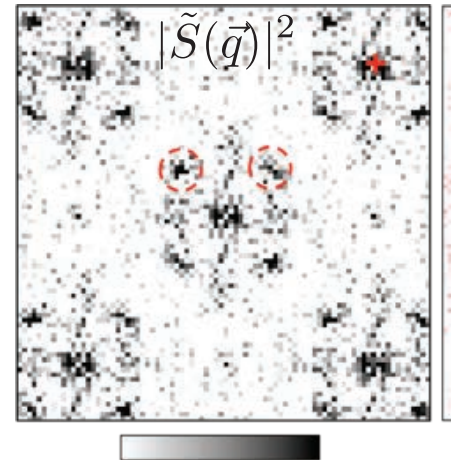

(a)

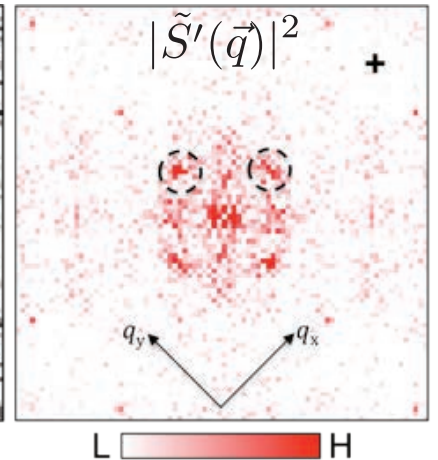

(b)

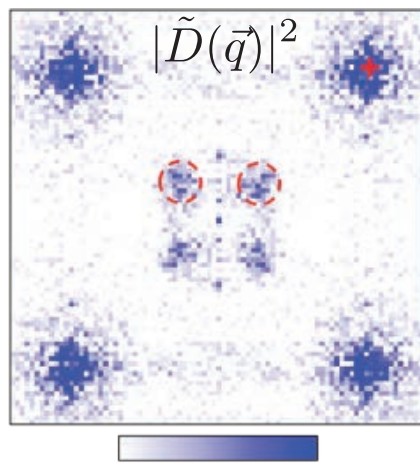

(c)

Figure 4.13: Power spectral densities (a) $\left|S^{Z}(\vec{q}, 18 \mathrm{meV})\right|^{2}$, (b) $\left|S^{\prime Z}(\vec{q}, 18 \mathrm{meV})\right|^{2}$, and (c) $\left|D^{Z}(\vec{q}, 88 \mathrm{meV})\right|^{2}$. The amplitude of the $s^{-}, s^{\prime}$ - and $d$-symmetry form factor components in a modulation at $\vec{Q}_{x, y}$ are calculated by integrating the weight inside the dashed circles for each of (a)-(c) respectively. The energies shown are chosen to be near where each of the form factor amplitudes are maximal. Bragg peak locations are marked by + .

The amplitude of each form factor component at a given energy can be quantified by summing the spectral weight inside the the red circles proximal to $\vec{Q}_{x, y}$ to yield energyresolved form factor amplitudes $S(|E|), S^{\prime}(|E|)$ and $D(|E|)$. As shown in figure 4.15, for $|E|<\Delta_{0}$, where dispersive Bogoliubov QPI modulations are observed, the $s$ - and $s^{\prime}$-symmetry form factors are dominant $\left(S(|E|), S^{\prime}(|E|)>D(|E|)\right)$. For $|E|>\Delta_{0}$, $S(|E|)$ and $S^{\prime}(|E|)$ diminish rapidly. In contrast $D(|E|)$ grows rapidly for $|E|>\Delta_{0}$ to a maximum at the pseudogap energy scale $|E|=\Delta_{1}$. This demonstrates that the nondispersive CDW modulations have a predominantly $d$-symmetry form factor, in accord 


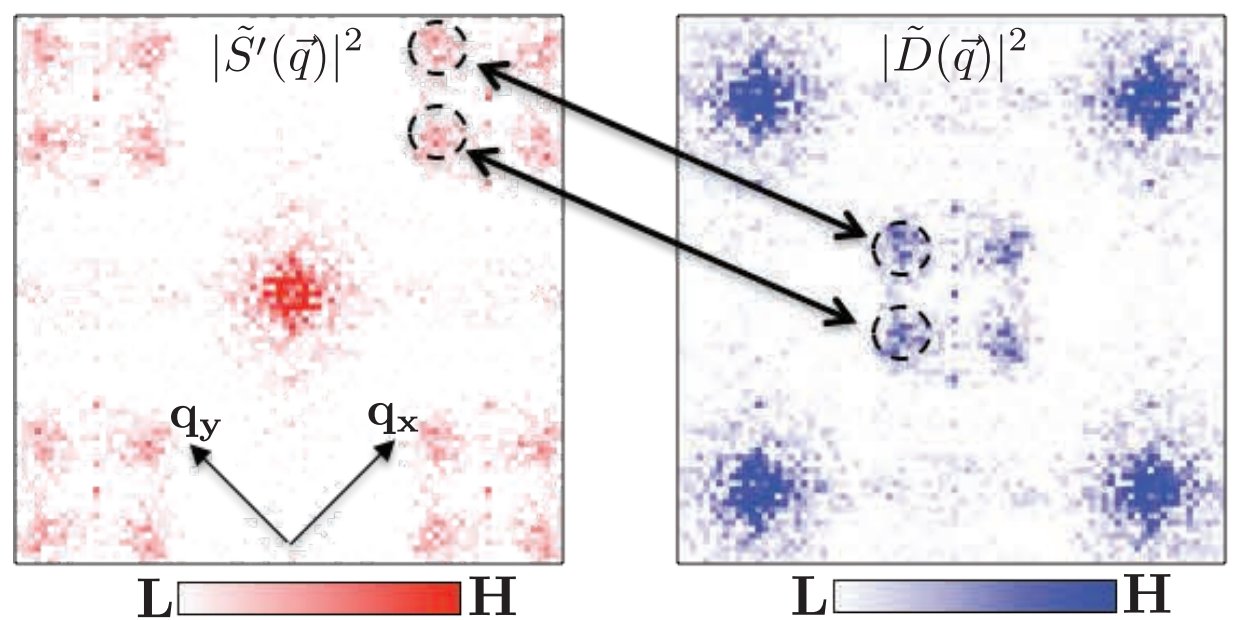

Figure 4.14: $\left|S^{\prime Z}(\vec{q}, 88 \mathrm{meV})\right|($ left $)$ and $\left|D^{Z}(\vec{q}, 88 \mathrm{meV})\right|$ (right). The black arrows demonstrate that the $d$-symmetry form factor modulations at wave-vector $\vec{Q}_{x, y}$ present $|E|=\Delta_{1}$ in underdoped $\mathrm{Bi}_{2} \mathrm{Sr}_{2} \mathrm{CaCu}_{2} \mathrm{O}_{8+\delta}$ manifest themselves in both $\left|S^{\prime Z}(\vec{q}, 88 \mathrm{mev})\right|$ and $\left|D^{Z}(\vec{q}, 88 \mathrm{meV})\right|$ but shifted by a reciprocal lattice vector.

with the analysis of the energy integrated function $R(\vec{r})$. Further, it demonstrates that the CDW results in modulations in the electronic structure which are maximal in amplitude for states with $|E| \approx \Delta_{1}$.

\subsection{Phase Relationship between the Modulation of Filled and Empty States}

Key information on the microscopic cause of the CDW is also contained in the relationship between modulations of states above and below the Fermi energy. To explore this issue in $\mathrm{Bi}_{2} \mathrm{Sr}_{2} \mathrm{CaCu}_{2} \mathrm{O}_{8+x}$, I show in figures 4.16 (a) and (b) the measured $g(\vec{r},+94 \mathrm{meV})$ from filled states and $g(\vec{r},-94 \mathrm{meV})$ from empty states respectively, each at the pseudogap energy scale $\Delta_{1}$ for this doping $(p \sim 6 \%)$.

For these two images the sub-lattice Fourier transform, $\tilde{D}^{g}(\vec{q}, E)$, is calculated and reveals a predominantly $d$-symmetry form factor modulation with wave-vectors near $\vec{Q}_{x}$ and $\vec{Q}_{y}$. Next, we use $\tilde{D}^{g}(\vec{q}, E)$ to determine the complex-valued functions $D_{x}^{g}(\vec{r})$ and $D_{y}^{g}(\vec{r})$. These encode the amplitude and spatial phase of the CDW modulation along $Q_{x}$ and $Q_{y} . D^{g}(\vec{q}, E)$ is Fourier filtered around the wave-vectors $\vec{Q}_{x, y}$ to determine the complex-valued $D_{x, y}^{g}(\vec{r})$, as detailed in section 2.8. Summarising, we take the full 


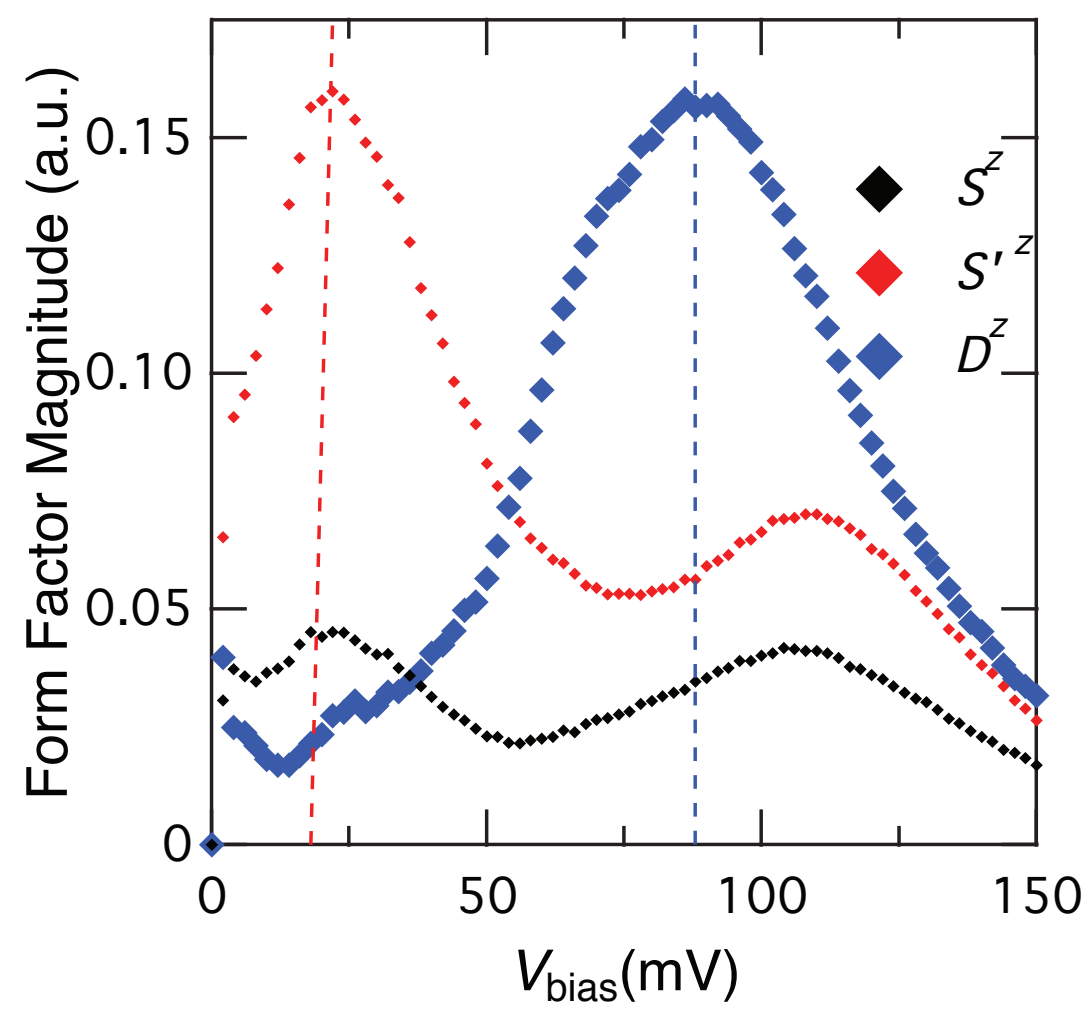

Figure 4.15: Measured energy dependence of form factor amplitudes $S(E)$ (black), $S^{\prime}(E)$ (red) and $D(E)$ (blue). To calculate these, $\vec{q}$ is integrated over the region inside the solid red circles in figure 4.13. These data reveal that the $d$-symmetry form factor amplitude is concentrated at energies surrounding $90 \mathrm{meV}$, which, at this hole density, is the independently measured pseudogap energy scale $\Delta_{1}$.

complex Fourier transform $D^{g}(\vec{q})$ and Fourier filter to create two complex real-space maps of $d$-symmetry form factor CDW amplitude at wave-vectors $\vec{Q}_{x, y}$,

$$
\begin{aligned}
& D_{x}(\vec{r})=\frac{2}{(2 \pi)^{2}} \int d \vec{q} e^{i \vec{q} \cdot \vec{r}} \tilde{D}(\vec{q}) e^{-\frac{\left(\vec{q}-\vec{Q}_{x}\right)^{2}}{2 \Lambda^{2}}} \\
& D_{y}(\vec{r})=\frac{2}{(2 \pi)^{2}} \int d \vec{q} e^{i \vec{q} \cdot \vec{r}} \tilde{D}(\vec{q}) e^{-\frac{\left(\vec{q}-\vec{Q}_{y}\right)^{2}}{2 \Lambda^{2}}}
\end{aligned}
$$

where $\Lambda^{-1}$ is the characteristic length scale over which variations in $D_{x, y}(\vec{r})$ can be resolved, and is set by the filter width in Fourier space. The phase of the CDW modulation along $\vec{Q}_{y}$, say, is then given by

$$
\phi_{y}(\vec{r}, E)=\arctan \left(\operatorname{Im} D_{y}^{g}(\vec{r}, E) / \operatorname{Re} D_{y}^{g}(\vec{r}, E)\right) .
$$

This is shown for $E=+94 \mathrm{meV}$ in figure 4.16 (c) and for $E=-94 \mathrm{meV}$ in figure 4.16 (d). Visual comparison reveals that these two $\phi_{y}(\vec{r}, \pm E)$ images are out of phase with each 


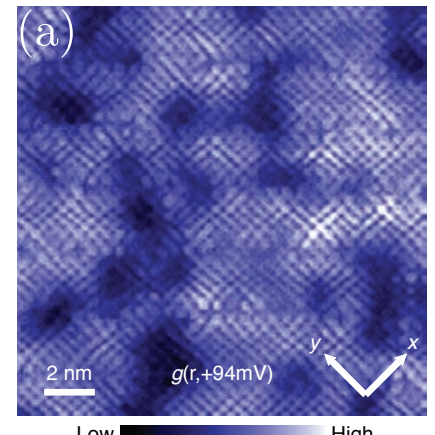

Low High

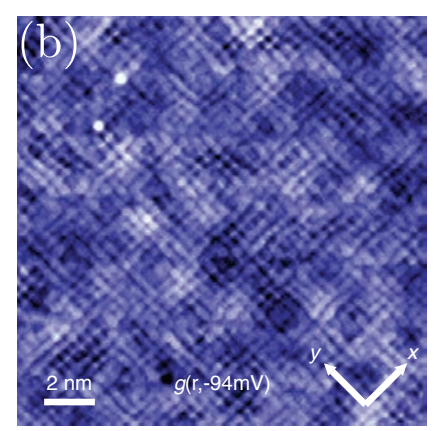

Low High

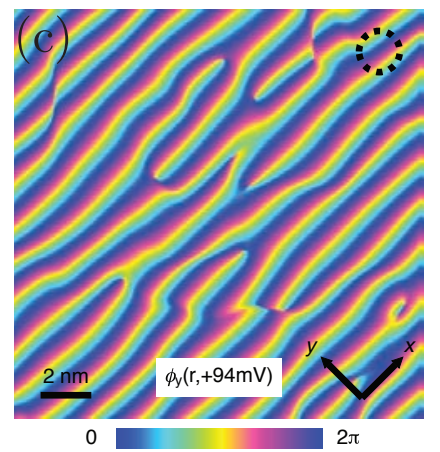

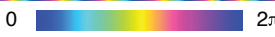

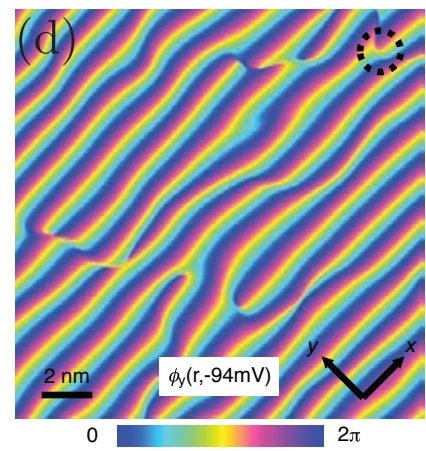

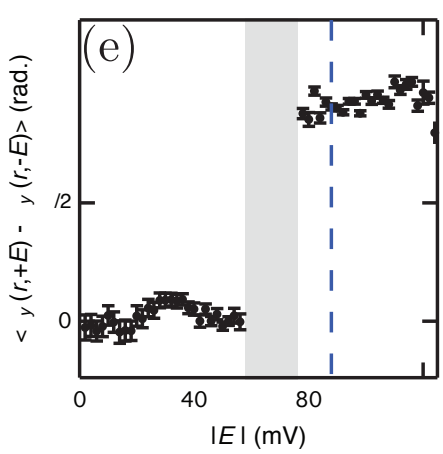

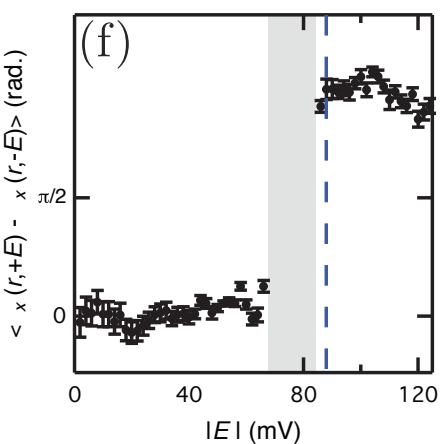

Figure 4.16: (a) Differential tunnelling conductance image $g(\vec{r},+94 \mathrm{meV})$. (b) Differential tunnelling conductance image $g(\vec{r},-94 \mathrm{meV})$. The colour scale is reversed compared to (a). (c) The spatial phase of the $d$-symmetry form factor CDW modulating along the y-direction, $\phi_{y}(\vec{r})$ is calculated using equation 4.21 from $g(\vec{r},+94 \mathrm{meV})$ shown in (a). The dashed circle shows the $\vec{r}$-space radius equivalent to the $\vec{q}$-space filter used to generate the $\phi_{x . y}(\vec{r}, E)$ images by Fourier filtering (see section 2.8). (d) The spatial phase of the $d$-symmetry form factor CDW modulating along the y-direction $\phi_{y}(\vec{r})$ is calculated using equation 4.21 from $g(\vec{r},-94 \mathrm{meV})$ shown in (b). (e) From the field of view of (a) and (b), we show the energy dependence of the relative phase of $g(\vec{r},+E)$ and $g(\vec{r},-E)$ modulations along the y-axis: $\phi_{y}(\vec{r},+E)-\phi_{y}(\vec{r},-E)$ when summed over every pair of identical pixel locations $\vec{r}$. (f) Similarly we show the relative phase of $g(\vec{r},+E)$ and $g(\vec{r},-E)$ for modulations along the x-axis: $\phi_{x}(\vec{r},+E)-\phi_{x}(\vec{r},-E)$. The low-energy modulations at $+E$ and $-E$ are in phase spatially and so have a relative phase difference of 0 . As the pseudogap energy, $\Delta_{1}(\approx 90 \mathrm{meV}$ at this doping), is approached the relative spatial phase of empty state and filled state CDW modulations varies wildly in the narrow energy grange shaded grey, and then quickly develops a robust phase shift of $\pi$.

other by $\pi$. Indeed, the spatial-average value of $\phi_{y}(\vec{r},+E)-\phi_{y}(\vec{r},-E)$ as a function of $E$ (over the whole field of view of figures 4.16 (a) and (b) ) is shown in figure 4.16 (e). It reveals that the relevant $\vec{Q}_{y}$ components of $g(\vec{r},+E)$ and $g(\vec{r},-E)$ are in phase with each other at low energy but rapidly evolve at $|E|>70 \mathrm{meV}$ and become $\pi$ out of phase at $|E| \sim \Delta_{1}$ (figures 4.16 (a) and (b)). The shaded region indicates evolution through a situation where some areas exhibit $\phi \sim 0$ and some $\phi \sim \pi$, but this is quickly resolved on reaching the pseudogap energy $\Delta_{1}$. In appendix F I demonstrate that this cannot be an effect of the "setup-effect" artifact described in chapter 2. 
Similar analysis of the phases $\phi_{x}(\vec{r}, \pm E)$ of $\vec{Q}_{x}$ modulations yields a virtually identical result (figure 4.16 (f)). These phenomena occur throughout the underdoped region of the $\mathrm{Bi}_{2} \mathrm{Sr}_{2} \mathrm{CaCu}_{2} \mathrm{O}_{8+\delta}$ phase diagram (see appendix $\mathrm{G}$ ). This demonstrates that a $\pi$ phase shift between CDW modulations in the density of states above and below the chemical potential at the energy scale $\Delta_{1}$ is a generic feature of the CDW in these materials.

\subsection{Doping-Dependence of the CDW Wave-vector}

Figure 4.12 shows the dispersion of the scattering vectors, $\vec{q}_{2}, \ldots . \vec{q}_{7}$, arising from Bogoliubov QPI for $|E|<\Delta_{0}$ (extracted from $\left|S^{\prime Z}(\vec{q},|E|)\right|$ ) as red points. As detailed in section 2.7 , these scattering vectors originate from scattering between the tips of "bananas" formed by equal energy contours of the Bogoliubon dispersion. These banana tips trace out the $\vec{k}$-space path of the ungapped Fermi surface $[27,87]$. As such, the octet of scattering vectors can be inverted to infer position of the underlying Fermi surface in $\vec{k}$-space.

It is known that for $\mathrm{Bi}_{2} \mathrm{Sr}_{2} \mathrm{CaCu}_{2} \mathrm{O}_{8+\delta}$ with $p \lesssim 0.19$ the tips of these bananas do not trace out a complete Fermi surface [29]. Rather, the QPI modulations, which extinguish at $|E| \approx \Delta_{0}$, trace out incomplete "Fermi-arcs". These arcs are shown for a series of hole concentrations in figure 4.17 (a).

For energies $|E|>\Delta_{0}$ the wave-vectors of $d$-symmetry form factor CDW modulations, $\left|\vec{Q}_{x, y}\right|$, (extracted from $\left.\left|D^{Z}(\vec{q},|E|)\right|\right)$ are shown as blue points. As stated above and as expected for a CDW, the modulations are non-dispersive over a wide range of energies. Adopting the common convention in x-ray studies [119-121] for estimating the CDW wave-vector magnitude $|\vec{Q}|$, we measure $\left|\tilde{D}^{Z}(\vec{q},|E|)\right|$ along lines in the high symmetry directions $(1,0)$ and $(0,1)$ that pass through the CDW peak and fit these data to a background plus Gaussian. The peak positions of the two Gaussians are then assigned to be the values of $\vec{Q}_{x}$ and $\vec{Q}_{y}$.

It is important to appreciate that a wide range of $\vec{Q}$ values can actually be detected under each peak in $\left|\tilde{D}^{Z}(\vec{q},|E|)\right|$ and in x-ray scattering intensities. It remains to be determined whether these broad peaks with incommensurate maxima are due to domains of continuously incommensurate CDW or domains of commensurate CDW separated by 
(a)

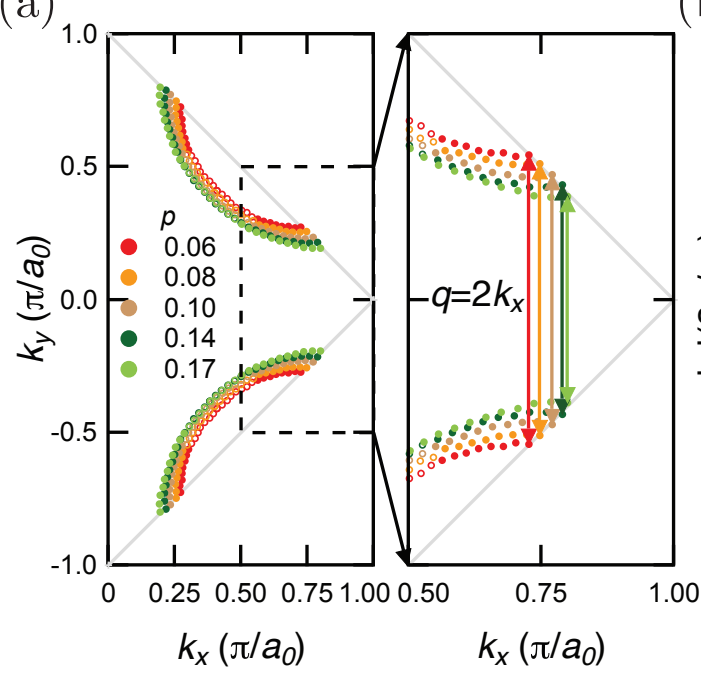

(b)

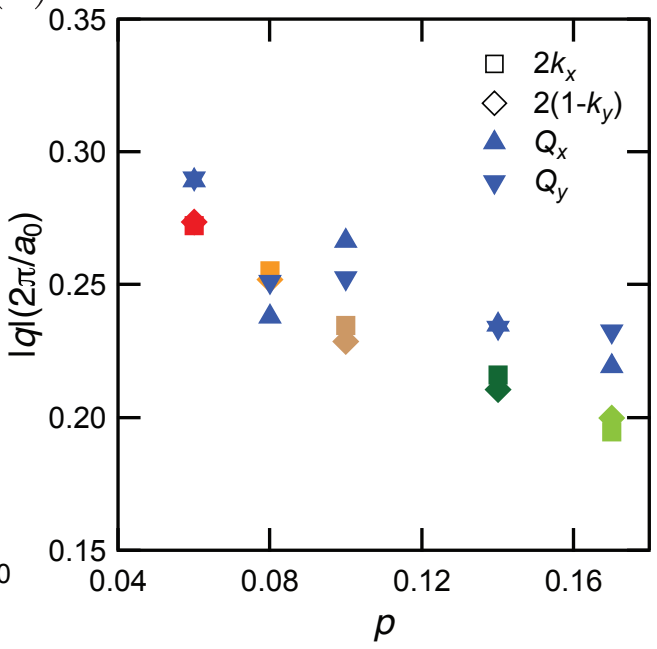

Figure 4.17: (a) Measured doping dependence of $\vec{k}$-space arcs traced out by Bogoliubov QPI vector $\vec{q}_{4}$ indicating the position of arc like sections of the Fermi surface. The Bogoliubov QPI is observed to terminate at the anti-ferromagnetic Brillouin zone boundary (grey line) for $p<0.19$ in $\mathrm{Bi}_{2} \mathrm{Sr}_{2} \mathrm{CaCu}_{2} \mathrm{O}_{8+\delta}$. Coloured double headed arrows indicate the doping dependence of the $\vec{q}$-vector linking tips of these arcs. (b) Doping dependence of the CDW $\vec{Q}_{x}$ and $\vec{Q}_{y}$ in underdoped $\mathrm{Bi}_{2} \mathrm{Sr}_{2} \mathrm{CaCu}_{2} \mathrm{O}_{8+\delta}$ as measured from the position of the peak maxima in $\left|\tilde{D}^{Z}\left(\vec{q}, \Delta_{1}\right)\right|$ is shown using blue symbols. The measured doping dependence of the $\vec{q}$-vector linking tips of arcs beyond which the signature of Bogoliubov QPI disappears (figure 4.12 and references $[29,112]$ ) is shown by all other colours.

discommensurations [129]. Indeed recent work in our group concludes that the latter is true $[130]$.

In figure 4.17 (b), the doping dependence of the CDW wave-vector is shown using blue symbols. It is a decreasing function of hole concentration in agreement with x-ray studies of $\mathrm{Bi}_{2} \mathrm{Sr}_{2} \mathrm{CaCu}_{2} \mathrm{O}_{8+\delta}, \mathrm{Bi}_{2-y} \mathrm{~Pb}_{y} \mathrm{Sr}_{2-x} \mathrm{La}_{x} \mathrm{CuO}_{6+\delta}, \mathrm{YBa}_{2} \mathrm{Cu}_{3} \mathrm{O}_{6+x}$ and $\mathrm{HgBa}_{2} \mathrm{CuO}_{4+\delta}$ [58]. On the same axes I also plot the magnitude of the shortest vector joining the tips of the arcs shown in figure 4.17 (a) for the same series of hole concentrations. There is reasonable quantitative agreement between these two evolutions. This trend is also reported in combined ARPES and x-ray studies linking the CDW wave-vector the the vector linking the tips of the Fermi-arcs observed in the pseudogap regime $\left(T>T_{c}\right)$ of $\mathrm{Bi}_{2-y} \mathrm{~Pb}_{y} \mathrm{Sr}_{2-x} \mathrm{La}_{x} \mathrm{CuO}_{6+\delta}$ [121]. In contrast, in $\mathrm{La}_{2} \mathrm{CuO}_{4}$ derived cuprates the wavevector increases with increasing hole doping, consistent with segregation into hole-rich conducting regions separated by insulating regions. 
These results are curious because they are in clear agreement with many of the predictions of Fermi-surface driven CDW instability whilst being in similarly clear disagreement with others. Specifically, for a CDW of wave-vector $\vec{Q}$ formed by nesting of two sections of Fermi surface for which $\vec{k}_{1}-\vec{k}_{2}=\vec{Q}$ over some finite domain, a gap should open at the Fermi energy in the nested regions. The resulting wave-functions would then form bonding/anti-bonding states below/above the Fermi level which are proportional to $e^{i \vec{k}_{1} \cdot \vec{r}} \pm e^{i \vec{k}_{2} \cdot \vec{r}}$. The local density of states would then exhibit spatial modulations given by $\left|e^{i \vec{k}_{1} \cdot \vec{r}} \pm e^{i \vec{k}_{2} \cdot \vec{r}}\right|^{2}=2(1 \pm \cos (\vec{Q} \cdot \vec{r}))$. Here modulations above and below the chemical potential are $\pi$ out of phase as is observed in our measurements.

However, it has been established that the doping evolution of the CDW wave-vector does not match that connecting the anti-nodal sections of the Fermi surface but rather the vector joining the tips of Fermi arcs [121]. If it were strong coupling between these eight points, or "hot-spots", that leads to CDW formation the CDW gap formed should be maximal at these points. Clearly what is observed is that this is exactly the point where the pseudogap closes $[24,27]$.

Nevertheless, that the CDW wave-vector in $\mathrm{Bi}_{2} \mathrm{Sr}_{2} \mathrm{CaCu}_{2} \mathrm{O}_{8+\delta}$, $\mathrm{Bi}_{2-y} \mathrm{~Pb}_{y} \mathrm{Sr}_{2-x} \mathrm{La}_{x} \mathrm{CuO}_{6+\delta}, \mathrm{YBa}_{2} \mathrm{Cu}_{3} \mathrm{O}_{6+x}$ and $\mathrm{HgBa}_{2} \mathrm{CuO}_{4+\delta}$ evolves in the same way as a vector linking sections of the Fermi surface suggests that the Fermi surface plays some role in determining it.

\subsection{Conclusions and Proposed Future Experiments}

In this chapter I have shown that the short-ranged CDW present in $\mathrm{Bi}_{2} \mathrm{Sr}_{2} \mathrm{CaCu}_{2} \mathrm{O}_{8+\delta}$ and $\mathrm{Na}_{x} \mathrm{Ca}_{2-x} \mathrm{CuO}_{2} \mathrm{Cl}_{2}$ has a predominantly $d$-symmetry form factor. While predicted for some time, this is the first CDW with a $d$-symmetry form factor to be observed in nature. Similar observations have also been made in $\mathrm{YBa}_{2} \mathrm{Cu}_{3} \mathrm{O}_{6+x}$ by x-ray scattering, which is in contrast to the predominantly $s^{\prime}$-symmetry form factor observed in $\mathrm{La}_{2-x} \mathrm{Ba}_{x} \mathrm{CuO}_{4+\delta}[114-116]$.

A $d$-symmetry form factor arises naturally in spin-fermion "hot-spot" models of the cuprates, where it has a deep connection with the $d$-wave superconductivity because they arise out of the same fermions and the same effective interaction [94-97, 101, 103, 104]. A $d$-symmetry form factor is also a natural partner to $d$-wave superconductivity in 
the sense that it acts to protect the coherence of nodal quasi-particles against scattering from the disordered CDW in so called "cold-spots" [118].

Another possible explanation for the observed $d$-symmetry form factor is the Coulomb repulsion between holes on the oxygen sites of the $\mathrm{CuO}_{2}$ plane, which acts to favour their unequal occupation within the unit cell, i.e a $d$-symmetry form factor $[98,102,105,106]$. The observation of a short ranged CDW in the electron doped cuprate $\mathrm{Nd}_{2-x} \mathrm{Ce}_{x} \mathrm{CuO}_{4}$, where carriers are first doped into bands derived from the $\mathrm{Cu} 3 \mathrm{~d}_{x^{2}-y^{2}}$ orbitals, provides a way to falsify this hypothesis [131]. If measurements sensitive to the CDW form factor reveal that it is predominantly $d$-symmetry in electron doped cuprates the hypothesis cannot be true.

I also showed in this chapter that the magnitude of the incommensurate CDW wavevector in $\mathrm{Bi}_{2} \mathrm{Sr}_{2} \mathrm{CaCu}_{2} \mathrm{O}_{8+\delta}$ decreases with increasing hole concentration, as also observed by x-ray studies of $\mathrm{Bi}_{2} \mathrm{Sr}_{2} \mathrm{CaCu}_{2} \mathrm{O}_{8+\delta}, \mathrm{Bi}_{2-y} \mathrm{~Pb}_{y} \mathrm{Sr}_{2-x} \mathrm{La}_{x} \mathrm{CuO}_{6+\delta}, \mathrm{HgBa}_{2} \mathrm{CuO}_{4+\delta}$ and $\mathrm{YBa}_{2} \mathrm{Cu}_{3} \mathrm{O}_{6+x}$ but in contrast to the doping dependence in $\mathrm{La}_{2} \mathrm{CuO}_{4}$ derived cuprates which shows the opposite trend [58]. This phenomenology clearly suggests a role for the Fermi surface geometry in determining the CDW wave-vector.

This chapter demonstrates that the charge order in $\mathrm{Bi}_{2} \mathrm{Sr}_{2} \mathrm{CaCu}_{2} \mathrm{O}_{8+\delta}$ and $\mathrm{Na}_{x} \mathrm{Ca}_{2-x} \mathrm{CuO}_{2} \mathrm{Cl}_{2}$ consists of nanoscale domains of unidirectional $d$-symmetry form factor CDW. Recent high-magnetic field measurements in $\mathrm{YBa}_{2} \mathrm{Cu}_{3} \mathrm{O}_{6+x}$ reveal a magneticfield induced CDW that is completely unidirectional $[55,56]$. This is suggestive that the $\mathrm{CuO}_{2}$ plane has an intrinsic instability to unidirectional ordering. A cautionary caveat is that $\mathrm{YBa}_{2} \mathrm{Cu}_{3} \mathrm{O}_{6+x}$ is structurally orthorhombic, and so posseses no symmetry between $\mathrm{Cu}-\mathrm{O}$ directions. I propose that measurements of magnetic field induced CDW in cuprates with a structural symmetry between the $\mathrm{Cu}-\mathrm{O}$ directions be performed with the aim of directly demonstrating this unidirectional tendency of the $\mathrm{CuO}_{2}$ plane. 



\section{Chapter 5}

\section{The Scanned Josephson Tunnelling Microscope}

The main results of this chapter are reported in "Direct Detection of a Cooper-pair Density Wave in $\mathrm{Bi}_{2} \mathrm{Sr}_{2} \mathrm{CaCu}_{2} \mathrm{O}_{8+\delta}$ ", Nature, 532, 343 (2016).

This chapter details the development of a scanned Josephson tunnelling microscope. Motivated by the search for a pair density wave in cuprates, we employ a high temperature $d$-wave superconducting STM tip operating at temperatures $<50 \mathrm{mK}[132]$.

Scanned Josephson tunnelling microscopy (SJTM) is a direct probe of the superconducting order parameter on the nanometer scale [133]. It utilises the tunnelling of Cooper-pairs between a superconducting STM tip and a superconducting sample to measure the Cooper-pair condensate directly. In contrast, traditional STM measures the spectrum of single-particle excitations. Prior to the development of SJTM, superconductivity could be probed on the nanometer scale only indirectly using STM (by measuring the single-particle tunnelling gap) or with atomic site sensitivity using NMR or $\mu \mathrm{SR}$.

There are many cases where the gap in the single-particle tunnelling spectrum is not sufficient to characterise spatial variations in superconductivity. Firstly, it is known that at magnetic impurities in $s$-wave superconductors the superconducting order parameter, $\Psi$, is suppressed whereas the single-particle gap, $\Delta$, is unperturbed [134-137]. Similarly, 
there is also a gap at the centre of superconducting vortices in cuprate superconductors where $\Psi \rightarrow 0$ [138] . Finally, there are pair density wave states which we would not expect to exhibit a modulation in $\Delta$ and thus cannot be unambiguously detected by STM.

In the face of these adversities a handful of groups have initiated efforts to develop SJTM as a direct probe of the superconducting order parameter [139-145]. In our group we have focussed on an implementation of SJTM using high temperature $d$-wave superconducting tips to search for a pair density wave in cuprate superconductors [132].

\subsection{Fundamentals of SJTM Operation}

The purpose of the scanned Josephson tunnelling microscope is to directly visualise spatial variations in the superconducting order parameter on the nanometer scale. To achieve this we employ a superconducting STM tip, as shown in figure 5.1 (a). In contrast to normal STM, this allows the tunnelling of Cooper pairs between the tip and a superconducting sample, otherwise known as the Josephson effect. As will be detailed in section 5.3, this tunnelling of Cooper pairs leads to a peak in the tunnelling current near zero voltage bias. The magnitude of this peak current can be used as a direct measure of the superconducting order parameter.

The superconducting tip also allows superconductor-insulator-superconductor (SIS) single-particle tunnelling between the tip and sample, as discussed in section 2.1.4. Thus, the same tip-sample junction can be used to measure both the superconducting order parameter and properties of the single-particle excitation spectrum in the sample, such as the superconducting gap energy or the energy of an impurity resonance.

SJTM operates like an enhanced version of the spectroscopic-imaging STM (SI-STM) that was discussed in chapter 2. As with SI-STM, junctions between the tip and sample will be established with set-point voltage and current, $V_{s}$ and $I_{s}$, on a fine twodimensional grid of points $\left\{\vec{r}_{i}\right\}$. At each of these points the STM feedback loop will be turned off upon attaining the set-point current and the tip-sample separation, $z_{i}$, recorded in the topograph, $T(\vec{r})$, as $T\left(\vec{r}_{i}\right)=z_{i}$. 


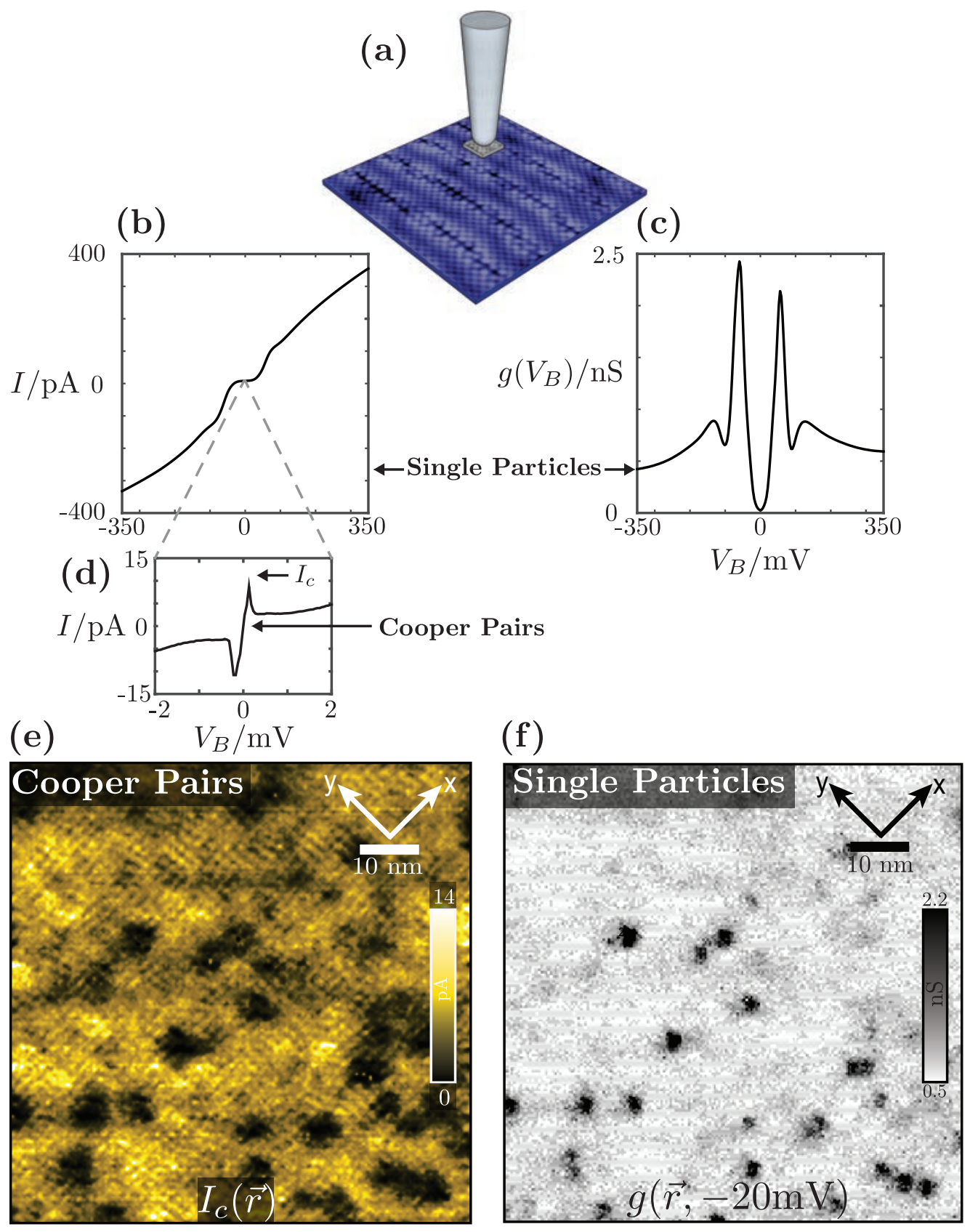

Figure 5.1: (a) A high temperature $d$-wave scanned Josephson tunnelling microscope (SJTM). A nanometer sized piece of $\mathrm{Bi}_{2} \mathrm{Sr}_{2} \mathrm{CaCu}_{2} \mathrm{O}_{8+\delta}$ is attached to a tungsten STM tip to form a superconducting STM tip. (b) A typical $I\left(V_{B}\right)$ characteristic for tunnelling between a $\mathrm{Bi}_{2} \mathrm{Sr}_{2} \mathrm{CaCu}_{2} \mathrm{O}_{8+\delta}$ sample and our $\mathrm{Bi}_{2} \mathrm{Sr}_{2} \mathrm{CaCu}_{2} \mathrm{O}_{8+\delta}$ nano-flake tip. For $\left|V_{B}\right|>1 \mathrm{mV}$ the current is carried almost entirely by single-particle tunnelling. (c) $g\left(V_{B}\right)=d I / d V_{B}$ corresponding to (b). (d) Enlargement of (b) near $V_{B}=0$ showing a region with far greater conductance than the single-particle channel that terminates in a peak current $I_{c}$. In this region the current is carried predominantly by Cooper pair tunnelling. (e) $I_{c}(\vec{r})$ map visualising variations in the superconducting order parameter. It is derived from $I\left(V_{B}\right)$ curves of the type shown in $(\mathrm{d})$, measured on a fine grid of points in the field of view. (f) $g(\vec{r},-20 \mathrm{mV})$ for the same field of view as (e), simultaneously visualising variations in the single-particle excitation spectrum. 
The bias voltage, $V_{B}{ }^{1}$, is then ramped and the current and differential conductance, $I\left(V_{B}\right)$ and $g\left(V_{B}\right)=d I / d V_{B}$, recorded. For each point on the surface of the sample, $I\left(V_{B}\right)$ and $g\left(V_{B}\right)$ such as those shown in figures 5.1 (b) and (c) are recorded. Combining the measurements from all points in the field of view yields the familiar spectroscopic maps $I\left(\vec{r}, E=e V_{B}\right)$ and $g\left(\vec{r}, E=e V_{B}\right)$.

For $\left|V_{B}\right|,\left|V_{s}\right|>1 \mathrm{mV}, T(\vec{r}), I\left(\vec{r}, E=e V_{B}\right)$ and $g\left(\vec{r}, E=e V_{B}\right)$ are entirely equivalent to those obtained using normal SI-STM, albeit with a more complicated tip density of states. This is because, in this voltage range, the current is carried almost entirely by single particles. Conversely, for $\left|V_{B}\right| \lesssim 10-100 \mu \mathrm{V}$ the current is carried predominantly by Cooper pairs. This allows us to extract another spectroscopic map, $I_{c}(\vec{r})$, which directly measures spatial variations in the superconducting order parameter.

In section 5.3 I will show that the expected signature of Cooper pair tunnelling is a steep quasi-linear increase in current away for $V_{B}=0$, reaching a maximum current $I_{c}$, before dropping sharply. Figure 5.1 (d) shows an enlarged portion of figure 5.1 (b) near $V_{B}=0$ that exhibits just this phenomenology. On increasing $V_{B}$ beyond the point where the current drops sharply, the current once again increases but with a much smaller gradient. Here the current is predominantly carried by single particles.

The Cooper pair tunnelling is confined to a small range of voltages around $V_{B}=0$ and exhibits a much larger differential conductance than the single-particle tunnelling channel (steeper gradient near $V_{B}=0$ in figure 5.1 (d)). This allows us to effectively separate single-particle and Cooper pair tunnelling contributions and hence simultaneously visualise superconducting order parameter and local density of states variations using the spectroscopic maps $I_{c}(\vec{r})$ and $g\left(\vec{r}, E=e V_{B}\right)$ respectively. $I_{c}(\vec{r})$ and $g\left(\vec{r}, E=e V_{B}\right)$ measured in the same field of view are shown in figures 5.1 (e) and (f). This ability to simultaneously and directly visualise both the ground state condensate and its single-particle excitation spectrum lies at the heart of SJTM's utility.

Having briefly described the utility and operation of SJTM I will now discuss how the Josephson effect may be used as a probe of the superconducting order parameter and how, for our ultra-small junctions, it leads to $I\left(V_{B}\right)$ characteristics of the form shown in figure $5.1(\mathrm{~d})$.

\footnotetext{
${ }^{1}$ In this chapter the bias voltage output by the STM electronic control unit will be denoted $V_{B}$ to distinguish it from the voltage dropped across the tunnelling junction $V_{J J}$. See section 5.3 for details.
} 


\subsection{The Josephson Effect as a Measure of the Supercon- ducting Order Parameter}

In chapter 1 I described superconductivity as being defined by the phase rigidity of a charged condensate. Perhaps the most striking physical manifestation of this phase rigidity is the Josephson effect [72]. Josephson realised that if two superconductors were coupled, for instance through a tunnel junction, that this phase rigidity should extend to coupling the phases of the two superconductors. This insightful, yet controversial, assertion led to him being awarded the Nobel Prize in 1973 following the experimental confirmation of his theory [146].

To examine the consequences of coupling between superconductors let us follow a simple phenomenological treatment before returning later to a microscopic description which is closer to Josephson's original reasoning. Consider two isolated superconductors with order parameters $\Psi_{1}=\left|\Psi_{1}\right| e^{i \theta_{1}}$ and $\Psi_{2}=\left|\Psi_{2}\right| e^{i \theta_{2}}$. Then introduce a coupling between these superconductors and consider its first symmetry allowed term in the free energy expansion,

$$
\begin{aligned}
F_{J} & =\frac{1}{2} \kappa\left(\Psi_{1} \Psi_{2}^{*}+\Psi_{2} \Psi_{1}^{*}\right) \\
& =\kappa\left|\Psi_{1}\right|\left|\Psi_{2}\right| \cos \left(\theta_{1}-\theta_{2}\right) \\
& =\kappa\left|\Psi_{1}\right|\left|\Psi_{2}\right| \cos (\phi),
\end{aligned}
$$

where $\kappa$ is a constant that characterises the strength of the coupling between the condensates.

The coupling between the superconductors depends linearly on the magnitude of each the superconducting order parameters and, crucially, on the difference in phase between them, $\phi$. Thus, this coupling term represents a rigidity to changes in the relative phase of the two superconductors.

However, equation 5.3 is not gauge invariant. To satisfy local gauge symmetry then under a transformation of the magnetic vector potential, $\vec{A}(\vec{r}, t) \rightarrow \vec{A}(\vec{r}, t)+\nabla \chi(\vec{r}, t)$, the phase 
of the superconductors and the electric potential, $\Phi(\vec{r}, t)$, must undergo transformations,

$$
\begin{aligned}
\theta_{1} & \rightarrow \theta_{1}-\frac{2 e}{\hbar} \chi\left(\vec{r}_{1}, t\right) \\
\theta_{2} & \rightarrow \theta_{2}-\frac{2 e}{\hbar} \chi\left(\vec{r}_{2}, t\right) \\
\Phi(\vec{r}, t) & \rightarrow \Phi(\vec{r}, t)-\frac{\partial \chi(\vec{r}, t)}{\partial t} .
\end{aligned}
$$

Thus to make our original coupling free energy in equation 5.3 invariant under local gauge transformations, we should replace $\phi$ with a gauge invariant phase difference,

$$
\gamma=\phi+\frac{2 e}{\hbar} \int_{1}^{2} \vec{A} \cdot d \vec{l}
$$

where the integral is along any line joining the two superconductors. This yields a coupling free energy,

$$
F_{J}=\kappa\left|\Psi_{1}\right|\left|\Psi_{2}\right| \cos (\gamma)
$$

which is invariant under local gauge transformations.

We can also use local gauge invariance to address the relationship between the phase difference and voltage, $V_{J J}$, across the junction. In the absence of a magnetic field, the voltage across the junction is given by the difference in the electric potential, $\Delta \Phi=$ $\Phi\left(\vec{r}_{2}\right)-\Phi\left(\vec{r}_{1}\right)$. If there is no voltage across the junction then $\phi$ should take the value that minimises equation 5.3 and there should be no time evolution of the phase. This must be true in any gauge. By reference to equations 5.4-5.6 one can see that the only way of relating $\phi$ and $\Delta \Phi$ that enforces this is through

$$
\frac{d \phi}{d t}=\frac{2 e}{\hbar} \Delta \Phi
$$

which one can verify is gauge invariant. 
To find a gauge invariant relationship between the phase difference and $V_{J J}$ we should now take the time derivative of the gauge invariant phase,

$$
\begin{aligned}
\frac{d \gamma}{d t} & =\frac{d \phi}{d t}+\frac{2 e}{\hbar} \frac{d}{d t} \int_{1}^{2} \vec{A} \cdot d \vec{l} \\
& =\frac{d \phi}{d t}-\frac{2 e}{\hbar} \int_{1}^{2}(\nabla \Phi+\vec{E}) \cdot d \vec{l} \\
& =\frac{d \phi}{d t}-\frac{2 e}{\hbar} \Delta \Phi-\frac{2 e}{\hbar} \int_{1}^{2} \vec{E} \cdot d \vec{l} .
\end{aligned}
$$

Then substituting for $\frac{d \phi}{d t}$ using the gauge-invariant relationship in equation 5.9 we obtain

$$
\frac{d \gamma}{d t}=\frac{2 e V_{J J}}{\hbar}
$$

This is one of Josephson's famous equations relating the rate of change of the gauge invariant phase to the voltage across the junction.

In chapter 1 I showed that phase rigidity in a superconductor led directly to the possibility of equilibrium supercurrents. Anticipating that phase rigidity in a Josephson junction will do the same we can calculate the reversible work done by an external current source in changing the phase difference across the junction using,

$$
\begin{aligned}
d F_{J} & =I_{p} V_{J J} d t \\
& =I_{p} \frac{\hbar}{2 e} \frac{\partial \gamma}{\partial t} d t
\end{aligned}
$$

and thus

$$
I_{p}=\frac{\hbar}{2 e} \frac{d F_{J}}{d \gamma} .
$$

Substituting for $F_{J}$ from equation 5.8 we then arrive at Josephson's other equation,

$$
I_{p}=I_{J} \sin (\gamma)
$$

where

$$
I_{J}=\frac{\hbar}{2 e} \kappa\left|\Psi_{1}\right|\left|\Psi_{2}\right|
$$

Remembering that this is an equilibrium current that must flow without dissipation, these equations imply that there is a phase-difference dependent Cooper pair current that can flow between the junctions without any voltage dropped, i.e a supercurrent. 
Importantly for the research presented in this thesis, the maximum supercurrent that can flow is proportional directly to $\left|\Psi_{1}\right|$ and $\left|\Psi_{2}\right|$. The significance of all of this to SJTM is that if one can measure the quantity $I_{J}$ as a function of position on a sample's surface, then it follows directly from equation 5.18 that we can map spatial variations in the sample's order parameter.

Recapping, we have made three assumptions:

1. Superconductors are phases of matter which break a global phase symmetry.

2. Local gauge-invariance is preserved.

3. The order parameters of the superconductors are allowed to weakly couple.

The consequences of these assumptions were the Josephson equations,

$$
\begin{aligned}
& I_{p}=I_{J} \sin (\phi) \\
& \frac{d \phi}{d t}=\frac{2 e V_{J J}}{\hbar},
\end{aligned}
$$

which I have chosen to write in a form that assumes $\vec{B}=0$, as I will not discuss magnetic fields in the remainder of this chapter. Thus, with a minimal number of assumptions we conclude that any SJTM measurement of $I_{J}$ will map spatial variations in the superconducting order parameter, regardless of the microscopic details of the condensate. The subject of sections 5.3 and 5.6 will be how to measure $I_{J}$ from the current-voltage characteristics of the ultra-small Josephson junctions used in SJTM when they are embedded in realistic experimental circuits.

The most famous objector to Josephson's idea was Bardeen. The essence of his objection was that assumption 3) above was violated. Quoting from reference [73] Bardeen writes:

"In a recent note, Josephson uses a somewhat similar formulation to discuss the possibility of superfluid flow across the tunnelling region, in which no quasi-particles are created. However, as pointed out by the author, pairing does not extend into the barrier, so that there can be no such superfluid flow." 
However, it is possible to microscopically demonstrate that the phase of two superconductors can couple through a tunnelling junction. The approach taken by Josephson was the tunnelling Hamiltonian formalism introduced in chapter 2 with left and right electrodes described by BCS wave-functions with gaps $\Delta_{1}=\left|\Delta_{1}\right| e^{i \theta_{1}}$ and $\Delta_{2}=\left|\Delta_{2}\right| e^{i \theta_{2}}$ and same the tunnelling Hamiltonian we used to calculate single-particle tunnelling rates,

$$
\hat{H}_{T}=\sum_{\vec{k}, \vec{q}} M_{\vec{k}, \vec{q}}\left(\hat{c}_{\vec{k} \sigma}^{\dagger} \hat{c}_{\vec{q} \sigma}+\hat{c}_{-\vec{q}-\sigma}^{\dagger} \hat{c}_{-\vec{k}-\sigma}\right)+H \cdot C
$$

where $\hat{c}_{\vec{k} \sigma}^{\dagger}$ creates an electron in state $\vec{k}$ with spin $\sigma$ on the left electrode and $\hat{c}_{\vec{q} \sigma}^{\dagger}$ creates an electron in state $\vec{q}$ with spin $\sigma$ on the right electrode [69].

Following the treatment of Anderson in reference [147], the second order perturbative correction to the ground state energy due to $\hat{H}_{T}$ at $T=0$ is given by

$$
\Delta E=-N_{1}(0) N_{2}(0)|M|^{2} \frac{\left|\Delta_{1}\right|\left|\Delta_{2}\right|}{\left|\Delta_{1}\right|+\left|\Delta_{2}\right|} K\left(\left|\frac{\left|\Delta_{1}\right|-\left|\Delta_{2}\right|}{\left|\Delta_{1}\right|+\left|\Delta_{2}\right|}\right|\right) \cos (\phi),
$$

where $N_{1}(0)$ and $N_{2}(0)$ are the densities of states of the two electrodes, $K(x)$ is a complete elliptical integral of the first kind, $\left|M_{\vec{k}, \vec{q}}\right|=|M|$ and terms independent of $\phi$ have been omitted. This microscopically establishes that, in as much as the tunnelling Hamiltonian formalism is a valid description of a tunnelling junction, there is a coupling between the phases of the two superconductors.

Comparing equation 5.22 to equation 5.3 and noting that the normal state resistance of the junction, $R_{N}$, is given by,

$$
\frac{1}{R_{N}}=\frac{4 \pi e^{2}}{\hbar}|M|^{2} N_{1}(0) N_{2}(0)
$$

one arrives at a expression for $I_{J}$ in terms of microscopic parameters of the superconductors:

$$
I_{J} R_{N}=\frac{2}{e} \frac{\left|\Delta_{1}\right|\left|\Delta_{2}\right|}{\left|\Delta_{1}\right|+\left|\Delta_{2}\right|} K\left(\left|\frac{\Delta_{1}-\Delta_{2}}{\Delta_{1}+\Delta_{2}}\right|\right)
$$

For non-zero temperature Ambegaokar and Baratoff found a similar analytic expression in the case where $\left|\Delta_{1}\right|=\left|\Delta_{2}\right|=|\Delta(T)|$ :

$$
I_{J} R_{N}=\frac{\pi|\Delta(T)|}{2 e} \tanh \left(\frac{|\Delta(T)|}{2 k_{B} T}\right) .
$$


We can make a connection between this expression and the phenomenological one in equation 5.18 using the work of Gor'kov, who showed that for $T \approx T_{c}, \Delta \propto \Psi$ [148]. Expanding equation 5.25 about $\Delta=0$ gives $I_{J} \propto|\Delta|^{2} \propto|\Psi|^{2}$. Equation 5.18 has the same dependence on $|\Psi|$, demonstrating the consistency of both approaches. For $T \rightarrow 0$ equation 5.25 yields $I_{J} \propto \Delta$. In this limit one can demonstrate consistency between equations 5.25 and 5.18 by noting that the superfluid density, $\rho_{s} \propto|\Psi|^{2}$, is proportional to $N(0) \Delta$ for $T \rightarrow 0$.

Later in this chapter I will present SJTM measurements of the cuprate superconductor $\mathrm{Bi}_{2} \mathrm{Sr}_{2} \mathrm{CaCu}_{2} \mathrm{O}_{8+\delta}$ which has a predominantly $d$-wave superconducting order parameter. The internal structure of the pair wave-functions will only enter into our phenomenological calculation of the Josephson current in the coupling constant $\kappa$, leaving the form of the Josephson equations unchanged. The value of $\kappa$ will depend on the pairing symmetry on either side of the junction, as well as details of the junction itself, such as its orientation with respect to the crystallographic axes of the superconductors.

$\breve{S}$ makov et al. calculated $I_{J}$ using the tunnelling Hamiltonian formalism for c-axis tunnelling between an $s$-wave superconductor with gap $\left|\Delta_{k}\right|=\left|\Delta_{0}\right|$ and a $d$-wave superconductor with gap $\left|\Delta_{k}\right|=\left|\Delta_{0}\right|\left(\cos \left(k_{x}\right)-\cos \left(k_{y}\right)\right)$, finding that $I_{J}=0^{2}$ [133]. Anticipating that $I_{J}$ will be highly suppressed for Josephson tunnelling between a $d$-wave superconductor and an $s$-wave superconducting tip our implementation of cuprate SJTM uses a $d$-wave tip, whose fabrication is detailed in section 5.5.

For a $d$-wave to $d$-wave junction, $I_{J}$ is sensitive to the relative orientation of the tip and sample order parameters. It is maximal when they are aligned and zero when rotated from each other by an azimuthal angle $\alpha=\frac{\pi}{4}$ [133]. In section 5.5 I present evidence that the crystallographic axes of our $\mathrm{Bi}_{2} \mathrm{Sr}_{2} \mathrm{CaCu}_{2} \mathrm{O}_{8+\delta}$ nano-flake tip are aligned with those of the sample in our measurements. Generally though, the relative orientation of the tip and sample only affects the absolute magnitude of $I_{J}$ and does not impact the ability of SJTM to measure relative spatial variations in $I_{J}$.

\footnotetext{
${ }^{2}$ Physical quantities are not identically zero by coincidence, this is a result of an underlying symmetry. Consider two tetragonal superconductors with order parameters $\Psi_{1}$ and $\Psi_{2}$ separated along their c-axis by a junction that is invariant under $90^{\circ}$ rotations about that axis such as vacuum or an amorphous insulator. The coupling free energy, $F_{J}=\frac{1}{2} \kappa\left(\Psi_{1} \Psi_{2}^{*}+\Psi_{2} \Psi_{1}^{*}\right)$, must be invariant under those same $90^{\circ}$ rotations. Consequently, if $\Psi_{1}$ and $\Psi_{2}$ transform as different irreducible representations of the tetragonal point group then $\kappa$ must be identically zero. Thus $\kappa$ is identically zero if $\Psi_{1}$ and $\Psi_{2}$ transform as $B_{1 g}$ $\left(\mathrm{d}_{x^{2}-y^{2}}\right)$ and $\mathrm{A}_{1 g}(s$-wave) representations respectively.
} 


\subsection{Josephson Current-Voltage Characteristics in Ultra- Small Junctions}

In the above I demonstrated that the intrinsic Josephson critical current, $I_{J}$, of a tunnelling junction formed between a superconducting sample and a superconducting STM tip can be used as a direct measure of the superconducting order parameter in the sample, regardless of microscopic details of the condensate. In SJTM we hope to measure $I_{J}$ using the current-voltage characteristic, $I\left(V_{J J}\right)$, of the tip-sample Josephson junction. However, the ultra-small tip-sample junctions required to attain nanometer resolution can have radically different current-voltage characteristics to the micron scale junctions most of us are familiar with. At the root of this are the strong phase fluctuations present in ultra-small junctions.

In the following I will discuss the physics of ultra-small Josephson junctions and their expected current-voltage characteristics under conditions applicable to SJTM. To this end, I will begin by reviewing the more familiar current-voltage characteristics of Josephson junctions with strong phase coupling, which are typically larger.

The material contained in this section is necessarily quite technical. While included here for logical flow, one way to use this material would be to refer back to it as necessary from the sections 5.4-5.8 which detail the experimental implementation and validation of SJTM.

\subsection{1 $I\left(V_{J J}\right)$ Characteristics for Josephson Junctions with Strong Phase Coupling}

The Josephson equations,

$$
\begin{aligned}
I_{p} & =I_{J} \sin \phi \\
\frac{d \phi}{d t} & =\frac{2 e}{\hbar} V_{J J},
\end{aligned}
$$

demonstrate that in order to calculate the current-voltage characteristic of a Josephson junction, $I\left(V_{J J}\right)$, we must first understand the dynamics of the phase difference, $\phi$, across the junction. 
We can model the dynamics of $\phi$ semi-classically using the parallel combination of a Josephson circuit element, a capacitance and a resistance as shown in figure 5.2. The Josephson element represents the tunnelling current of Cooper-pairs and obeys equation 5.26a. The capacitance, $C_{J}$, is that between the electrodes and the resistance, $R$, represents their single-particle tunnelling channel. This model is known as the Resistively and Capacitively Shunted Junction (RCSJ) model [149, 150].

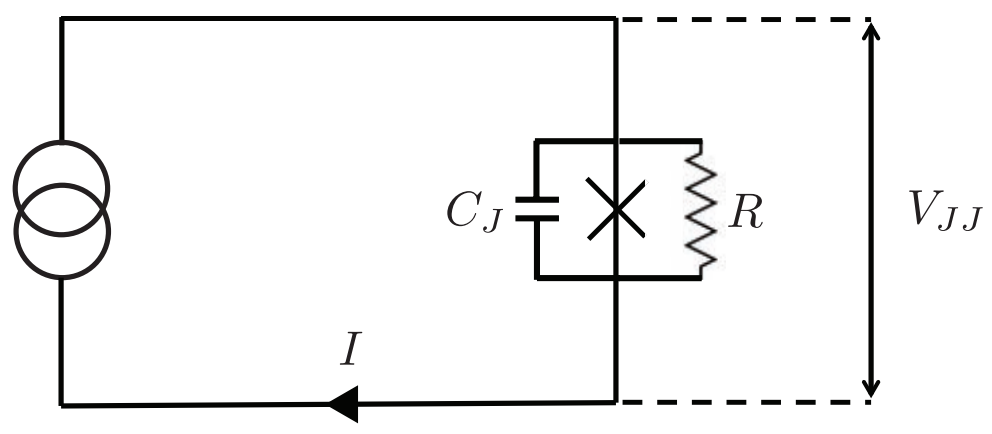

FIgURE 5.2: Circuit diagram for the Resistively and Capacitively Shunted Junction (RCSJ) model. A Josephson circuit element carrying a Cooper-pair current is current biased in parallel with a capacitance, $C_{J}$, and ohmic resistance, R. For an SJTM experiment, $C_{J}$ is between the tip and sample and $\mathrm{R}$ represents the single-particle tunnelling channel of the junction (which would in reality be non-ohmic). A voltage, $V_{J J}$, develops across the junction as a result of the current bias, $I$.

The equation of motion for $\phi$,

$$
I=I_{J} \sin \phi+\frac{V_{J J}}{R}+C_{J} \frac{d V_{J J}}{d t}
$$

is obtained by conservation of current. Substituting for $V_{J J}$ from equation $5.26 \mathrm{~b}$ we obtain

$$
\frac{d^{2} \phi}{d \tau^{2}}+\frac{1}{Q} \frac{d \phi}{d \tau}+\sin \phi=\frac{I}{I_{J}},
$$

where $\tau=\omega_{p} t, Q=\omega_{p} R C_{J}$ and $\omega_{p}=\sqrt{\frac{2 e I_{J}}{\hbar C}}$. In the limit where $\phi(\bmod 2 \pi) \rightarrow 0$ this describes a damped driven harmonic oscillator with quality factor $Q$ and resonant frequency $\omega_{p}$, known as the Josephson plasma frequency. In analogy to a mechanical system, the capacitance provides the inertia, the shunt resistance the damping and the Josephson element is the "spring".

If we bias the junction with a DC current, the dynamics of $\phi$ have an intuitive mechanical analogue. When $I(t)=I_{\mathrm{DC}}$ equation 5.28 describes a massive particle, moving with 
viscous drag, through a potential,

$$
U(\phi)=-E_{J} \cos \phi-\frac{\hbar I_{\mathrm{DC}}}{2 e} \phi
$$

resembling a "tilted washboard". $E_{J}=\hbar I_{J} / 2 e$, the Josephson energy, is the potential barrier between adjacent minima in this Josephson potential. The famous zero voltage state of the DC Josephson effect corresponds to the particle resting in metastable equilibrium in a minimum of the potential. We will use this washboard analogy, depicted in figure 5.3, to understand the different dynamic regimes of Josephson tunnelling and their $I\left(V_{J J}\right)$ characteristics.

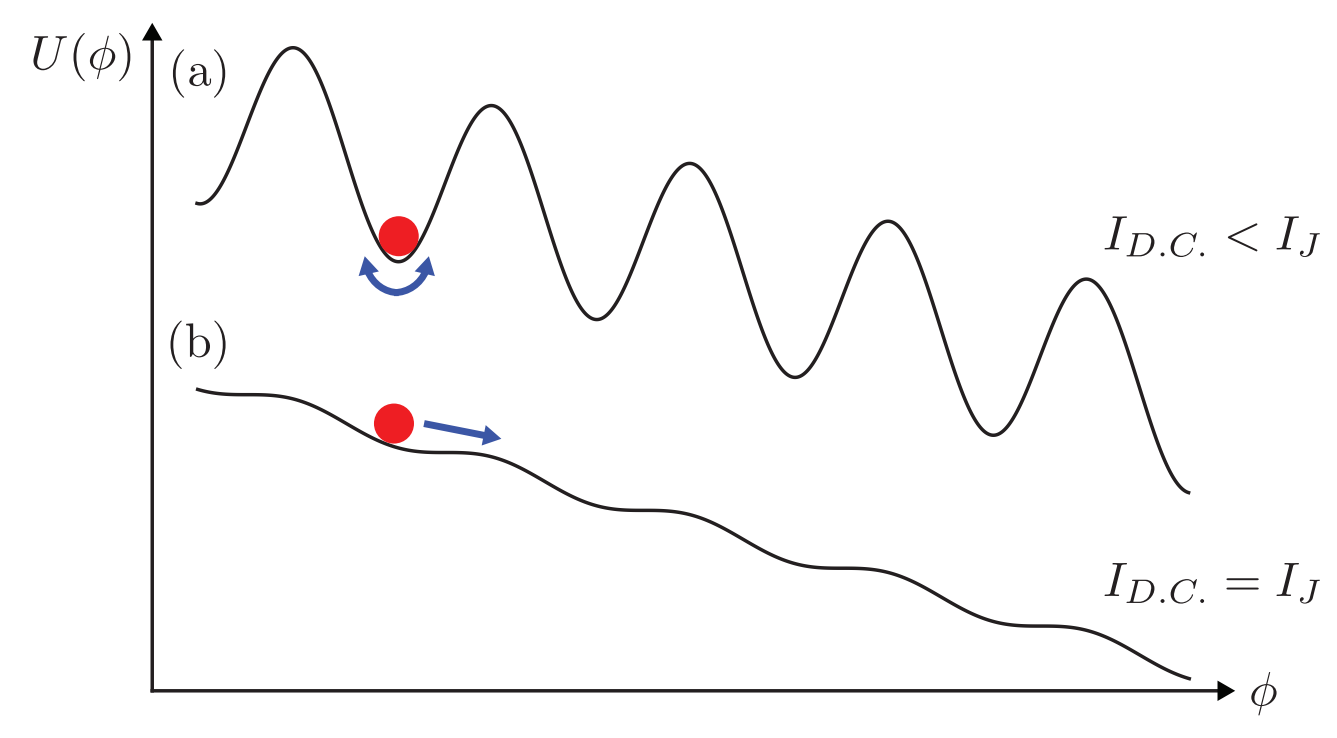

FiguRE 5.3: The tilted washboard potential of the RCSJ model presented in fig. 5.2 for the cases (a) $I_{\mathrm{DC}}<I_{J}$ and (b) $I_{\mathrm{DC}}=I_{J}$. The red circle symbolises a fictitious point-particle whose dynamics, under gravity and viscous damping, are analogous to that of the $\phi$.

As for a mechanical oscillator, the dynamical regimes of a Josephson junction are determined by its quality factor $Q$. If $Q^{2} \gg 1$ the junction is under-damped and its dynamics are dominated by its capacitance. In the mechanical analogy this means that the work done by gravity in moving the particle to an adjacent minimum of the washboard potential is much greater than the energy dissipated by the viscous damping. Conversely, if $Q^{2} \ll 1$ the junction is over-damped and the phase dynamics are viscous, with the velocity of the particle directly proportional to the force on it. Whether the dynamics are over or under-damped will be critical for the form of the $I\left(V_{J J}\right)$ characteristic. 


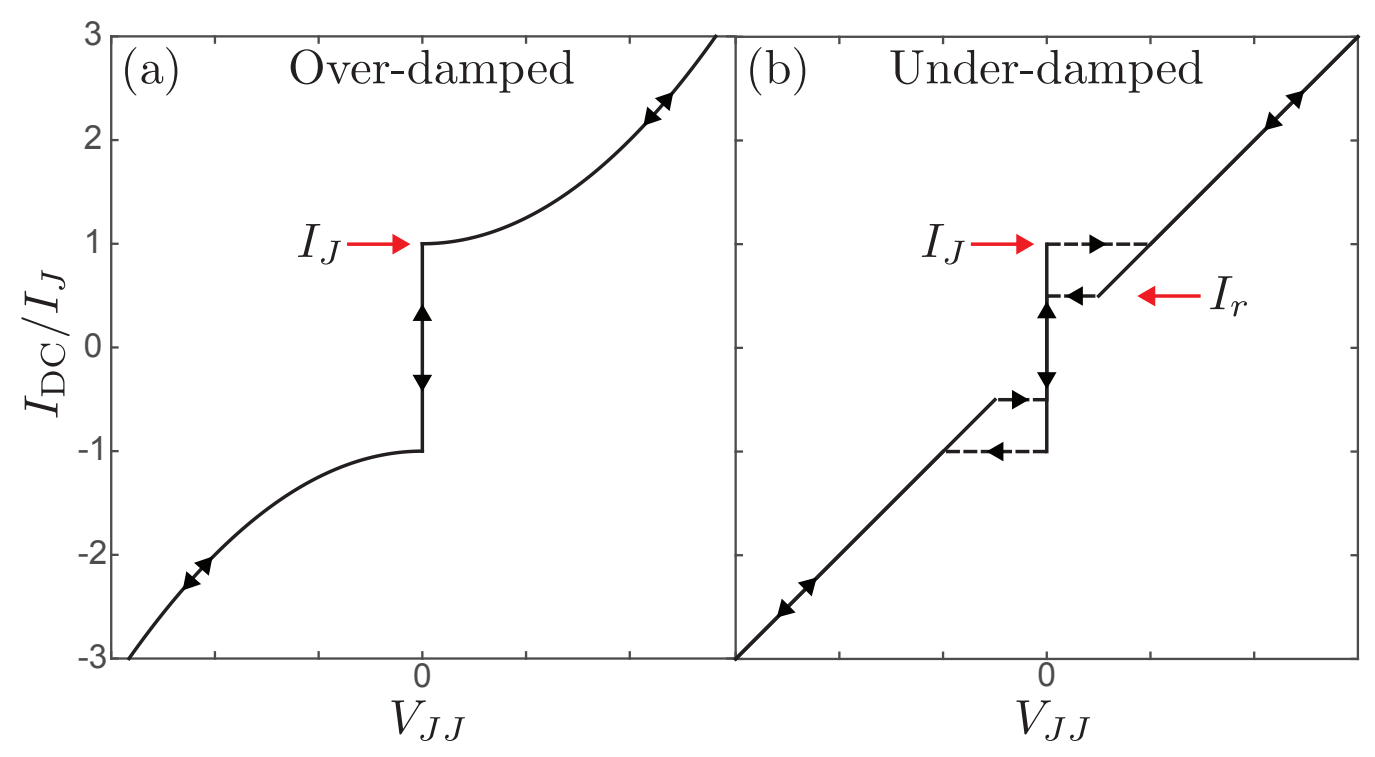

FiguRE 5.4: $I\left(V_{J J}\right)$ characteristic for Josephson junctions with coherent phase dynamics when they are (a) over-damped and (b) under-damped. In the case of the under-damped junction there is significant hysteresis with a difference between the Josephson critical current, $I_{J}$, and the re-trapping current, $I_{r}$.

As the DC current bias in increased, the slope of the washboard potential increases. For $I_{\mathrm{DC}}<I_{J}$ the phase will remain stationary in a minimum of the potential. Equation $5.26 \mathrm{~b}$ tells us that this means $V_{J J}=0$. As such, all current will flow without resistance through the Josephson junction and none will flow through the shunt resistance. When $I_{\mathrm{DC}}=I_{J}$ there ceases to be a minimum in the potential and the particle will roll down the slope resulting in $V_{J J} \neq 0$ and current flowing through the shunt resistance as shown in figure 5.4. This behaviour is the same regardless of whether the junction is over-damped or under-damped.

The $I\left(V_{J J}\right)$ characteristics of the under and over-damped regimes differ when $I_{\mathrm{DC}}$ is subsequently decreased. For the over-damped junction, where the phase has negligible inertia, it will become re-trapped as soon as $I_{\mathrm{DC}}<I_{J}$ and a minimum exists in the potential. The re-trapped current, $I_{r}$, equals $I_{J}$ and there is no hysteresis in the $I\left(V_{J J}\right)$ curve.

For the under-damped junction, the inertia of the mass is sufficient to carry it over the maxima in the potential that appear for $I_{\mathrm{DC}}<I_{J}$. For a current $I_{r}<I_{J}$, the damping will be sufficient to re-trap the phase in a minimum of the potential and the voltage 
across the junction will return to zero. This results in the significant hysteresis shown in figure 5.4(b).

For real Josephson junctions fluctuations in $\phi$ will inevitably result from thermal voltage noise $[151,152]$. For a junction biased with current $I_{\mathrm{DC}}<I_{J}$ the phase will oscillate around a minimum in the tilted-washboard potential. A sufficiently strong thermal fluctuation in $\phi$ will knock the phase into an adjacent minimum. The subsequent dynamics of the junction will depend upon the value of $Q^{2}$.

For an under-damped junction with $Q^{2} \gg 1$ the phase will continue to roll down the washboard potential, resulting in premature switching to the resistive state of the junction. For the over-damped junction with $Q^{2} \ll 1$ the phase will be re-trapped in the adjacent minimum and the junction will return to the zero-resistance state. The phase slip will give rise to a voltage pulse.

If the phase dynamics of a Josephson junction are over-damped and phase fluctuations sufficiently strong that these phase slip events occur frequently, then the time-average of the associated voltage pulses will result in a non-zero DC voltage across the junction. Consequently the resistance for the Cooper pair current through the junction will be small but non-zero.

A general criterion for whether a zero-resistance DC Josephson current will be observable can be obtained by comparing the height of the potential barrier between adjacent minima in the Josephson potential, $2 E_{J}$, to the characteristic energy of thermal fluctuations, $k T$. A zero-resistance DC Josephson effect will only be observed if

$$
\frac{E_{J}}{k T} \gg 1
$$

For the ultra-small junctions used in SJTM, $E_{J}$ is typically $\lesssim k T$. Consequently, no zero-resistance DC Josephson effect will be observed. However, its turns out that we are still able to measure a Cooper pair current in these junctions. From their $I\left(V_{J J}\right)$ characteristics we can extract the intrinsic critical current, $I_{J}$, and hence measure the superconducting order parameter in our sample. I will now move on to discuss the form we expect these $I\left(V_{J J}\right)$ characteristics to take and how $I_{J}$ can be extracted from them. 


\subsubsection{Thermal Phase Fluctuations in Ultra-Small Josephson Junctions}

The ultra-small size of SJTM junctions means that they have $I_{J} \sim 1 \mathrm{nA}$ which corresponds to an $E_{J} / k$ of $\sim 10 \mathrm{mK}$. In the experimentally accessible temperature range we will have $E_{J} \lesssim k T$. Consequently, the DC Josephson effect presented in figure 5.4 will typically not be observed in SJTM. In section 5.3.3 I will discuss how this can be true even at $T=0$ due to quantum phase fluctuations arising from the junction's charging energy.

Another facet of the ultra-small size of SJTM junctions is that they can have a junction capacitance, $C_{J}$, as small as $\sim 1 \mathrm{fF}$ and a shunt resistance provided by the SIS single-particle tunnelling channel of $\sim 0.1-100 \mathrm{M} \Omega$ [139-145]. On the face of it the small capacitance would favour over-damping while the large resistance favours underdamping. However, the single-particle tunnelling channel presented by $R\left(V_{J J}\right)$ in figure 5.2 is not the relevant characteristic resistance in this problem.

The frequency scale of phase slippage and re-trapping events is given by the Josephson plasma frequency, $\omega_{p}$, which is typically $\sim 10^{11} \mathrm{~Hz}$. At these frequencies the damping of ultra-small Josephson junctions used in SJTM experiments will be dominated by the shunt impedance of the bias circuitry and not the DC resistance of the single-particle tunnelling channel [74]. This shunting of the junction by its electromagnetic environment is given a lumped circuit representation in figure 5.5 by including a frequency dependent shunt impedance $Z(\omega)$ in parallel with the junction.

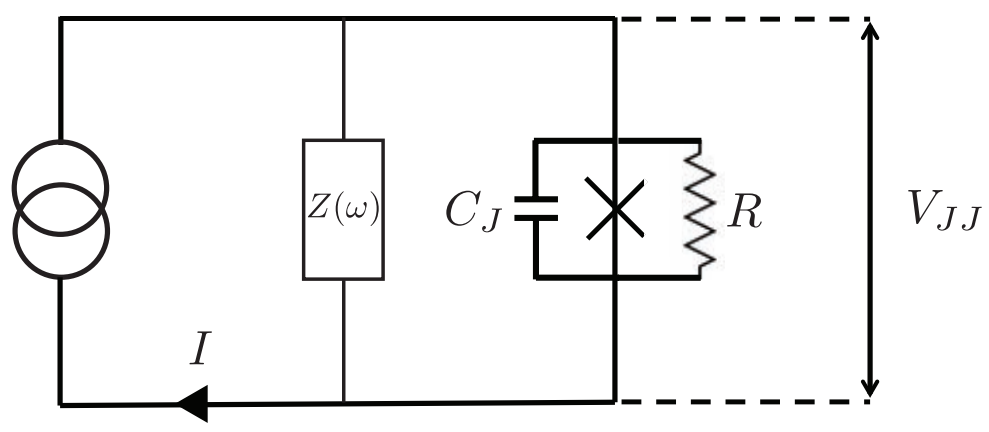

Figure 5.5: A modified circuit diagram for a current biased RCSJ model that is shunted by an frequency dependent impedance $Z(\omega)$.

At frequencies near DC, $Z(\omega)$ is effectively infinite. However, for frequencies $\sim 10^{11} \mathrm{~Hz}$ the junction can dissipate energy by radiating microwaves. In this frequency range 
the shunting impedance of the leads attached to the junction can be modelled as an infinite transmission line [153-156]. This is justified because the impedance to radiating microwaves will be controlled by the first few millimeters ( $\sim$ one wavelength) of the leads around the junction and any discontinuities in the leads will occur on a length scale much greater than this.

The high-frequency impedance of such a transmission line is given by $Z_{1} \sim \frac{Z_{0}}{2 \pi} \sim 100 \Omega$, where $Z_{0}$ is the impedance of free space [153-156]. This real, dissipative impedance is much less than that provided by the single-particle tunnelling channel and leads to the junction being over-damped at high-frequencies.

This over-damping of SJTM junctions at high frequencies allows the observation of a Cooper-pair current even if $E_{J} \lesssim k T$. In this case, using the tilted washboard analogy, the rate of hopping to adjacent minima will be large but the over-damping will ensure that the phase is re-trapped during each event. This allows the dynamics to be modelled as a biased random-walk with the phase diffusing down the washboard potential. The smaller barrier height for hopping down the washboard will lead to a time-average phase velocity $\langle\dot{\phi}\rangle$ and hence DC voltage $\frac{\hbar}{2 e}\langle\dot{\phi}\rangle$.

Before calculating the $I\left(V_{J J}\right)$ expected from this phase-diffusion process we must first address whether the junction is under current or voltage bias conditions. While this may at first seem to be obvious from the type of source used in the circuit, there is a subtlety that arises from the parasitic capacitance of the leads attached to the junction.

In figure 5.6 (a) I take the ideal current biased circuit in figure 5.5, explicitly include a parasitic capacitance between the junction leads, $C_{P}$, and replace the ideal current source by a voltage source in series with a large biasing resistor $R_{B}{ }^{3}$. In SJTM experiments $C_{J}$ can be as small as $\sim 1 \mathrm{fF}$ and $C_{P}$ is typically $\sim 1 \mathrm{nF}$. As a result, the voltage across $C_{P}$ can be well approximated as independent of time even though the current flowing through the Josephson junction consists of current spikes corresponding to tunnelling events [155].

This time-independence of the voltage across $C_{P}$ renders the junction effectively voltage biased. Thus, in modelling the behaviour of the junction we can replace the circuit in

\footnotetext{
${ }^{3}$ Note that although in going from figure 5.5 to figure 5.6 (a) the impedance $Z(\omega)$ has been moved from being in parallel with the junction to in series with it, this still represents a high-frequency dissipative shunt because it is in series with the junction capacitance $C_{J}$.
} 
figure 5.6 (a) by that in figure 5.6 (b). In this scheme the actual voltage source and biasing resistor are replaced by an effective voltage source with potential difference $V_{J J}$ across it. $V_{J J}$ must be determined self-consistently from the DC current flowing through the junction, i.e $V_{J J}=V_{B}-R_{B} I\left(V_{J J}\right)$.

(a)

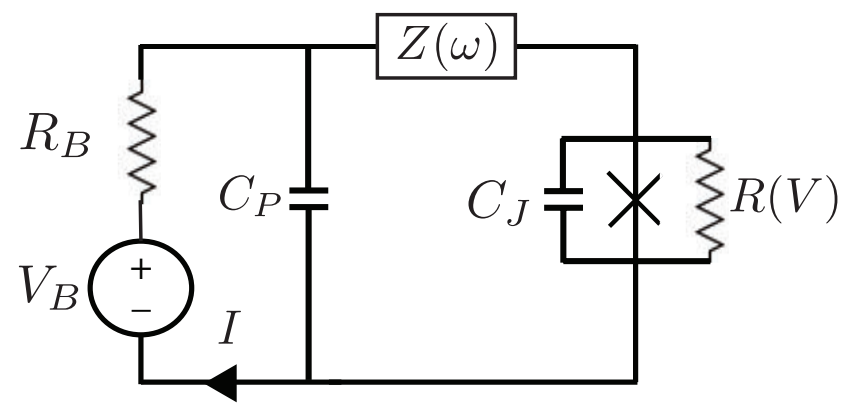

(b)

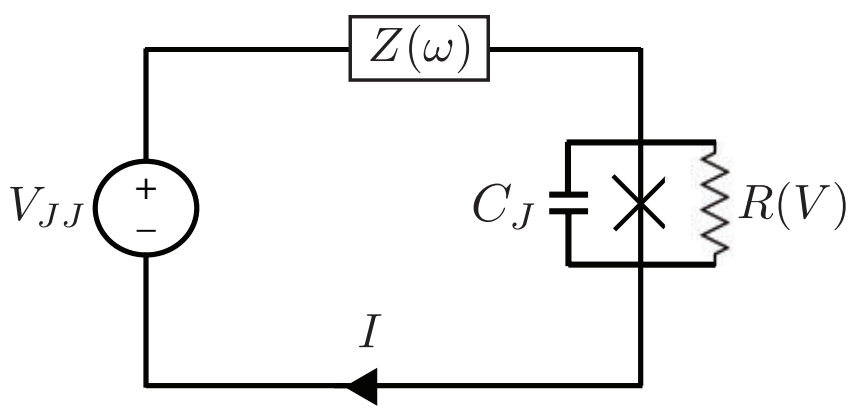

Figure 5.6: (a) An RCSJ model is biased by a non-ideal current source consisting of a voltage source in series with a large biasing resistance $R_{B}$. A parasitic capacitance, $C_{P}$, and a frequency dependent impedance, $Z(\omega)$, representing the electromagnetic environment of the junction are included. For an SJTM experiment $C_{P}$ is typically $\gg C_{J}$. As a result the voltage across the junction is approximately time-independent and it may be regarded as effectively voltage biased as shown in (b).

To calculate the $I\left(V_{J J}\right)$ characteristic for a junction in the phase-diffusion regime Ivanchenko and Zi'lberman used a circuit as in figure 5.6 (b) with $R(V)=\infty$ and real impedance, $Z(\omega)=Z$, in thermal equilibrium at temperature $T$ acting as source of Johnson voltage noise across the junction [152]. In the limit where $E_{J} \ll k T$ and $C \rightarrow 0$, the solution to the resulting stochastic equation is

$$
I_{p}\left(V_{J J}\right)=\frac{I_{J}^{2} Z}{2} \frac{V_{J J}}{V_{J J}^{2}+V_{C}^{2}},
$$

where $V_{C}=2 e Z k T / \hbar$. These $I_{p}\left(V_{J J}\right)$ curves are plotted in figure 5.7. Note that the subscript $p$ has been applied to denote that this current arises entirely from the flow of 
Cooper pairs.

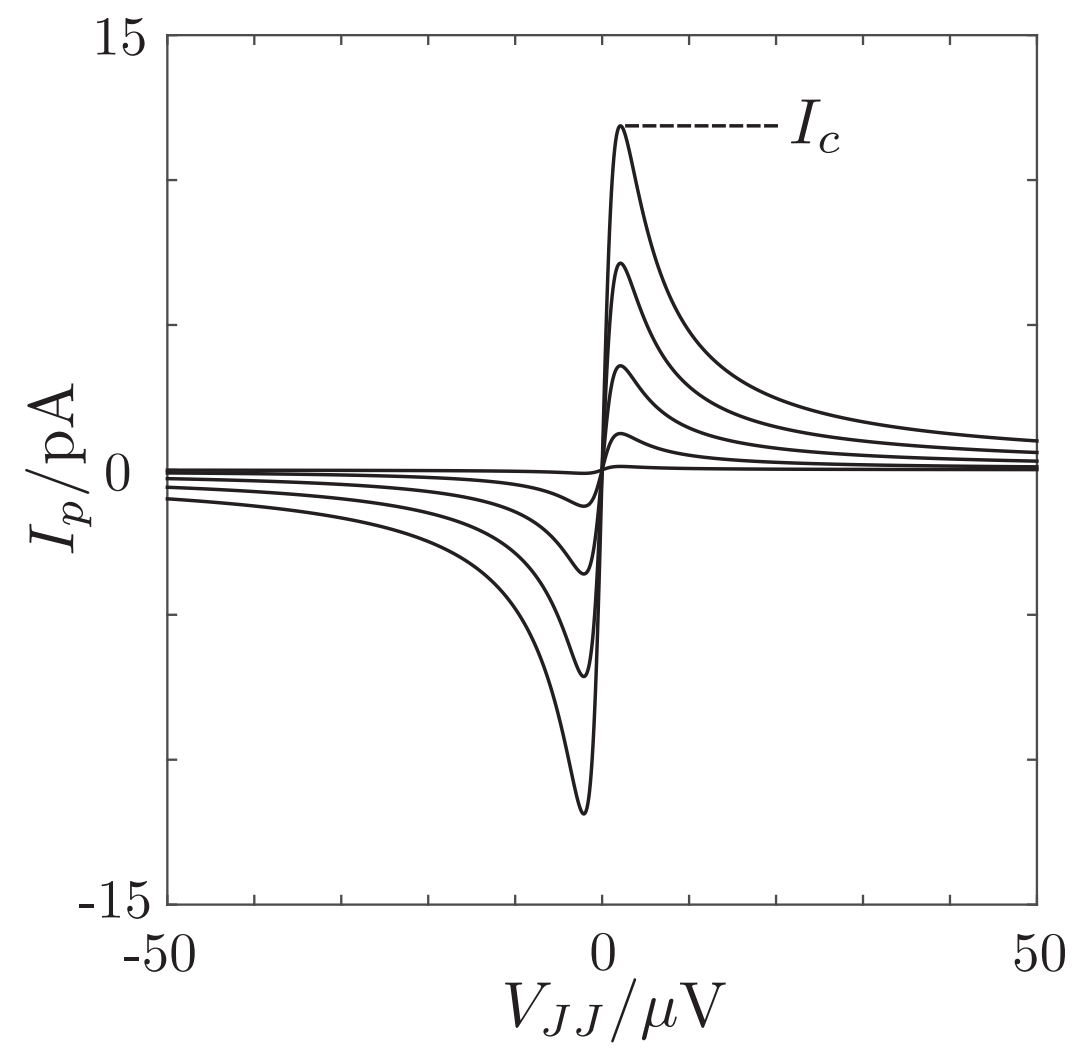

FIGURE 5.7: $I\left(V_{J J}\right)$ characteristics for an over-damped junction in the presence of strong thermal fluctuations. The curves plotted correspond to equation 5.31 with $T=$ $500 \mathrm{mK}, Z=100 \Omega$ and $I_{J}=100 \mathrm{pA}-1 \mathrm{nA}$ in equal increments. $I_{c} \propto I_{J}^{2}$ denotes the maximum Cooper pair current.

There are several pertinent features of these $I_{p}\left(V_{J J}\right)$ curves. Firstly, as $I_{J}$ is decreased the average diffusive phase velocity increases and with it the resistance of the junction near $V_{J J}=0$. Comparing figures 5.7 and 5.4 we can see that the effect of strong phase fluctuations has been to give a finite slope to the $I_{p}\left(V_{J J}\right)$ curves at $V_{J J}=0$. Secondly, they exhibit a peak in the Cooper-pair current at non-zero voltage, $V_{C}$, whose magnitude, $I_{c}$ is proportional to $I_{J}^{2}$. This fact is crucial for the operation of SJTM because measuring spatial variations in $I_{c}$ allows one to measure spatial variations in $I_{J}$ and hence the superconducting order parameter.

\subsubsection{Including both Thermal and Quantum Phase Fluctuations}

In the previous section I treated the dynamics of the phase classically, despite its quantum origin. The conclusion was that strong thermal fluctuations in $\phi$ lead to $I_{p}\left(V_{J J}\right)$ 
curves that have a finite slope at $V_{J J}=0$ and a peak Cooper-pair current proportional to $I_{J}^{2}$. In this section I will take into account quantum fluctuations of the phase resulting from the Coulomb charging of the junction and find that these properties of $I_{p}\left(V_{J J}\right)$ persist.

In the limit of strong fluctuations of the phase difference across a Josephson junction there is no DC Josephson effect. However, one can still measure $I_{J}$ through the current that arises from incoherent tunnelling of Cooper pairs between the electrodes. As the Josephson effect is often described as the tunnelling of Cooper pairs, the distinction between incoherent Cooper pair tunnelling and the coherent tunnelling that occurs in the standard Josephson effect warrants some explanation.

Consider the following model Hamiltonian of a junction between two superconducting electrodes labelled 1 and 2, each with optimum Cooper pair number, $N_{0}$, that minimises its electrostatic self-energy,

$$
\hat{H}=E_{C} \hat{N}^{2}-E_{J} \cos (\hat{\phi})
$$

where $\hat{N}=\hat{N}_{1}-\hat{N}_{2}$ is the Cooper pair number imbalance operator. $E_{C}=2 e^{2} / C_{J}$ is the charging energy of the junction and $E_{J}=\hbar I_{J} / 2 e$ is the Josephson energy. Note that $\hat{N}$ and $\hat{\phi}$ do not commute and obey an uncertainty relationship $\Delta N \Delta \phi \leq \frac{1}{2}$.

Let's work in the basis of number projected BCS wave-functions $|N\rangle_{1}$ and $|N\rangle_{2}$ for the electrodes. Writing the Josephson term in the form,

$$
E_{J} \cos (\hat{\phi})=\frac{E_{J}}{2}\left(e^{i \hat{\theta_{2}}} e^{-i \hat{\theta_{1}}}+e^{-i \hat{\theta_{2}}} e^{i \hat{\theta_{1}}}\right)
$$

and noting that $e^{-i \hat{\theta}_{i}}|N\rangle_{i}=|N+1\rangle_{i}$, we see that this term acts to transfer a Cooper pair between the two electrodes. Consequently, it has vanishing expectation if the state of the two electrodes has a definite number of Cooper pairs on each electrode.

In the limit where $E_{J} \gg E_{C}$ the ground state will be well approximated by a coherent superposition of product states with total Cooper pair number $2 N_{0}$ but different numbers of Cooper pairs on each electrode,

$$
|\Psi\rangle=\sum_{j} \alpha_{j}\left|N_{0}-j\right\rangle_{1}\left|N_{0}+j\right\rangle_{2}
$$


so as to give the ground state a definite phase difference [74]. Thus, the ground state energy is phase difference dependent and this phase rigidity will result in a phase dependent equilibrium Cooper pair current, i.e the Josephson effect. This is what I will term coherent Cooper pair tunnelling because the ground state of the junction has coherence between states that differ by the transfer of a Cooper pair between the electrodes.

In the opposite limit where $E_{C} \gg E_{J}$ the ground state is well approximated by a product state with a definite number of Cooper pairs on each electrode,

$$
|\Psi\rangle=\left|N_{0}\right\rangle_{1}\left|N_{0}\right\rangle_{2}
$$

so as to minimise the charging energy of the junction. The expectation of the Josephson term is then zero, resulting in the absence of a phase dependence to the ground state energy and consequently no equilibrium Josephson current.

However, we can regard the Josephson term as a Cooper pair tunnelling Hamiltonian and treat it perturbatively using Fermi's golden rule. At $T=0$ the Cooper pair tunnelling rate from electrode 1 to 2 is given by,

$$
\Gamma_{1 \rightarrow 2}=\frac{2 \pi}{\hbar}\left|\left\langle N_{0}+\left.1\right|_{2}\left\langle N_{0}-\left.1\right|_{1} E_{J} e^{-i \hat{\phi}} \mid N_{0}\right\rangle_{1} \mid N_{0}\right\rangle_{2}\right|^{2} \delta\left(\mu_{1}-\mu_{2}\right)
$$

where $\mu_{1}$ and $\mu_{2}$ are the chemical potentials for Cooper pairs in the two electrodes. This process I will refer to as incoherent Cooper pair tunnelling.

Two important points arise from this. In contrast to single-particle tunnelling there is not a continuum of initial and final states. Thus $\Gamma_{1 \rightarrow 2}$ is only non-zero when $\mu_{1}-\mu_{2}=$ $2 e V_{J J}=0$, where $V_{J J}$ is the voltage across the junction. But in this case $\Gamma_{1 \rightarrow 2}=$ $\Gamma_{2 \rightarrow 1}$ by symmetry and there is no net Cooper pair tunnelling current. The only way that a net Cooper pair current can occur is if Cooper pairs tunnel inelastically, by exchanging energy $2 e V_{J J}$ with their environment when they tunnel, as depicted in figure ??. This means that the exact form of $I_{p}\left(V_{J J}\right)$ will depend sensitively on the details of the interaction with the environment.

However, what we can tell from equation 5.36 is that the current will scale with $E_{J}^{2} \propto I_{J}^{2}$. It is this property of incoherent Cooper pair tunnelling that will allow us to measure $I_{J}$ in ultra-small SJTM junctions despite the absence of a coherent Josephson effect. Note that this is exactly the same scaling behaviour exhibited by the classical phase diffusion 


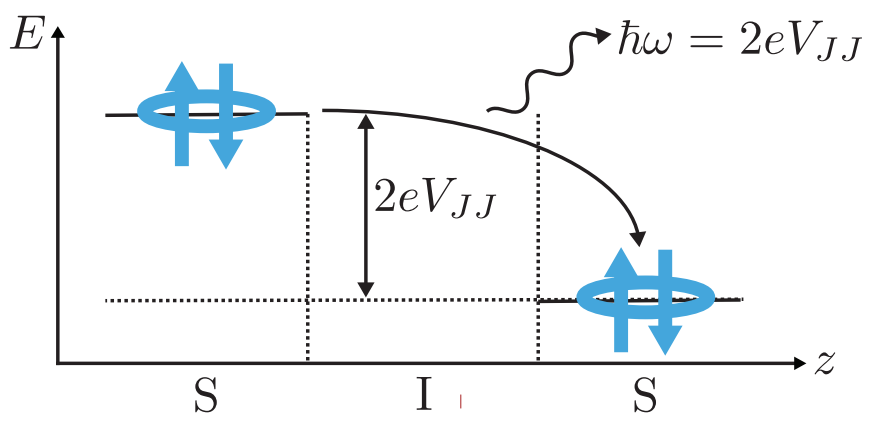

Figure 5.8: A Cooper-pair (blue) tunnels to the condensate on the other side of a potential barrier under voltage bias $V_{J J}$ by emitting a photon of energy $\hbar \omega=2 e V_{J J}$.

model in the limit of strong thermal phase fluctuations. This is a result of the fact that in both cases, phase fluctuations mean that states with a different number inbalance of Cooper pairs on the electrodes are combined only incoherently and hence the Cooper pair current arises from incoherent tunnelling.

What follows below is a extension of the argument above to include the effects of finite temperature and the electromagnetic environment. I will follow the treatment of Devoret et al. [157] who treated single-particle tunnelling junctions as open quantum systems and coupled them to a Caldera-Leggett model for the electromagnetic environment. This approach was subsequently extended to Josephson junctions in references [158-161]. Full details of the calculation below can be found in reference [155].

A Hamiltonian for the Josephson junction embedded in its environment is given by,

$$
\hat{H}=\hat{H}_{0}-E_{J} \cos (\hat{\phi})+\hat{H}_{\mathrm{env}},
$$

where $\hat{H}_{0}$ describes the Cooper pair condensates in the electrodes and the environment Hamiltonian,

$$
\hat{H}_{\mathrm{env}}=\frac{\hat{Q}^{2}}{2 C_{J}}+\sum_{n=1}^{\infty}\left[\frac{\hat{q}_{n}^{2}}{2 C_{n}}+\left(\frac{\hbar}{e}\right)^{2} \frac{1}{2 L_{n}}\left(\hat{\tilde{\phi}}-\hat{\phi}_{n}\right)^{2}\right],
$$

encodes the the electrostatic charging energy of the junction capacitance and the coupling to a dissipative reservoir [161]. Here $\hat{Q}$ is the charge on the junction capacitance $C_{J}$. Note that in using the operator $\hat{\tilde{\phi}}=\hat{\phi}-\frac{2 e}{\hbar} V_{J J} t$ we are subtracting the trivial time dependence of $\phi$. The reservoir in this open quantum system is modelled as an infinite 
number of LC resonators of resonant frequency, $\omega_{n}=1 / \sqrt{L_{n} C_{n}}$, bi-linearly coupled to the phase difference across the junction. Models for the reservoir of this type were first introduced by Caldera and Leggett [162].

If $E_{J} \ll E_{C}$ or $k T$ then we may treat the term $-E_{J} \cos (\phi)$ perturbatively. Applying Fermi's golden rule the forward tunnelling rate is given by,

$$
\Gamma_{i \rightarrow f}=\frac{2 \pi}{\hbar}\left|\left\langle f\left|E_{J} e^{-i \hat{\phi}}\right| i\right\rangle\right|^{2} \delta\left(E_{i}-E_{f}\right)
$$

where the initial and final states $|i\rangle$ and $|f\rangle$ are the combined states of the electrodes and the environment, with $E_{i}$ and $E_{f}$ their corresponding energies. Tracing out the environmental degrees of freedom as detailed in reference [155] the forward tunnelling rate is given by,

$$
\vec{\Gamma}=\frac{E_{J}^{2}}{4 \hbar^{2}} \int_{-\infty}^{\infty} d t \exp \left(i \frac{2 e}{\hbar} V t\right)\left\langle e^{\hat{\tilde{\phi}}(t)} e^{\hat{\tilde{\phi}}(0)}\right\rangle
$$

with the average $\langle\ldots\rangle$ taken with respect to the partition function $\operatorname{Tr}\left\{\exp \left(-\hat{H}_{\text {env }} / k T\right)\right\}$. This can be rewritten in the more compact form,

$$
\vec{\Gamma}=\frac{E_{J}^{2}}{4 \hbar^{2}} P(2 e V)
$$

where,

$$
P(E)=\frac{1}{2 \pi \hbar} \int_{-\infty}^{\infty} d t \exp \left[J(t)+\frac{i}{\hbar} E t\right],
$$

with phase correlation function $J(t)=\langle[\tilde{\tilde{\phi}}(t)-\hat{\tilde{\phi}}(0)] \hat{\tilde{\phi}}(0)\rangle$. $P(E)$ can be interpreted as the probability of a Cooper pair exchanging energy $E$ with the environment.

Using the Caldera-Leggett model in equation 5.38 for the environment, $J(t)$ can be expressed in terms of a single parameter $Z_{t}(\omega)=\left[i \omega C_{J}+Z^{-1}(\omega)\right]^{-1}$ to be

$$
J(t)=2 \int_{-\infty}^{\infty} \frac{d \omega}{\omega} \frac{\operatorname{Re} Z_{t}(\omega)}{R_{Q}}\left(\frac{e^{-i \omega t}-1}{1-e^{-\hbar \omega / k T}}\right)
$$

where $Z_{t}(\omega)$ is the input impedance of the environment and $R_{Q}=h / 4 e^{2}$ is the quantum of resistance. Assuming a knowledge of the total impedance, $Z_{t}(\omega)$, then the tunnelling current is given by the difference between the forwards and backwards tunnelling rates 
of Cooper-pairs:

$$
I_{p}\left(V_{J J}\right)=2 e\left[\vec{\Gamma}\left(2 e V_{J J}\right)-\vec{\Gamma}\left(-2 e V_{J J}\right)\right]=\frac{\hbar \pi e I_{J}^{2}}{4 e}\left[P\left(2 e V_{J J}\right)-P\left(-2 e V_{J J}\right)\right]
$$

This is a simple and powerful expression for the Cooper pair tunnelling current $I_{p}$. Its physical interpretation is the difference in probability for a Cooper pair to emit energy or absorb energy $2 e V_{J J}$ into the environment, multiplied by a pre-factor $\frac{\hbar \pi I_{J}^{2}}{4 e}$ which depends only on the superconducting order parameters in the electrodes and the strength of coupling between them.

\subsubsection{Expected Pair-Current $I_{P}(V)$ in Ultra-Small Josephson Junctions}

The details of $Z_{t}(\omega)$ for the junctions used in SJTM experiments are in general difficult to know or control. However, as discussed in section 5.3.2, at high frequencies the leads attached to the junction provide an impedance well approximated as ohmic and equal to $Z_{1} \approx Z_{0} / 2 \pi \approx 100 \Omega$. In this case, at $T=0, P(E)$ is sharply peaked at $E=0$ as shown in figure 5.9 (a) and equal to zero for $E<0$ [155].

Non-zero temperatures redistribute weight in $P(E)$ to larger voltages, with the weight at negative energies given by the principle of detailed balance, $P(-E)=e^{-\beta E} P(E)$, as shown schematically in figure 5.9 (b). From equation 5.44 the pair current can then be directly calculated as shown in figure 5.9 (c).

Figure 5.9 (c) is extremely similar to the $I\left(V_{J J}\right)$ curves in figure 5.7 that were calculated in the presence of strong classical phase fluctuations. Both exhibit a resistance at $V_{J J}=0$ that increases with decreasing $I_{J}$ as well as a peak in the pair current whose magnitude is proportional to $I_{J}^{2}$. This reflects the fact that the effects of both quantum and thermal phase fluctuations are roughly the same. They both prohibit coherent Cooper pair tunnelling and thus the pair current must flow through incoherent Cooper pair tunnelling.

In an SJTM experiment we wish to measure spatial variations in $I_{J}$ because it is directly proportional the superconducting order parameter in the sample. We will impose a constant temperature and $Z_{t}(\omega)$, being dominated by the measurement apparatus rather than sample, will also remain constant. Relative spatial variations in $I_{J}$, and hence $\Psi$ 
can thus be mapped by picking any feature of the $I\left(V_{J J}\right)$ curve that is easy to identify experimentally and taking the current or derivative at this point. A sensible experimental strategy is to measure the spatial variations in the peak Cooper-pair current, which we will call $I_{c}$. Using equation 5.44 we see that $I_{c} \propto I_{J}^{2}$. Having established in section 5.2 that $I_{J} \propto|\Psi|$, this shows us that using SJTM to measure $I_{c}$ as a function of position will yield a spatial map of relative variations in $|\Psi|^{2}$.
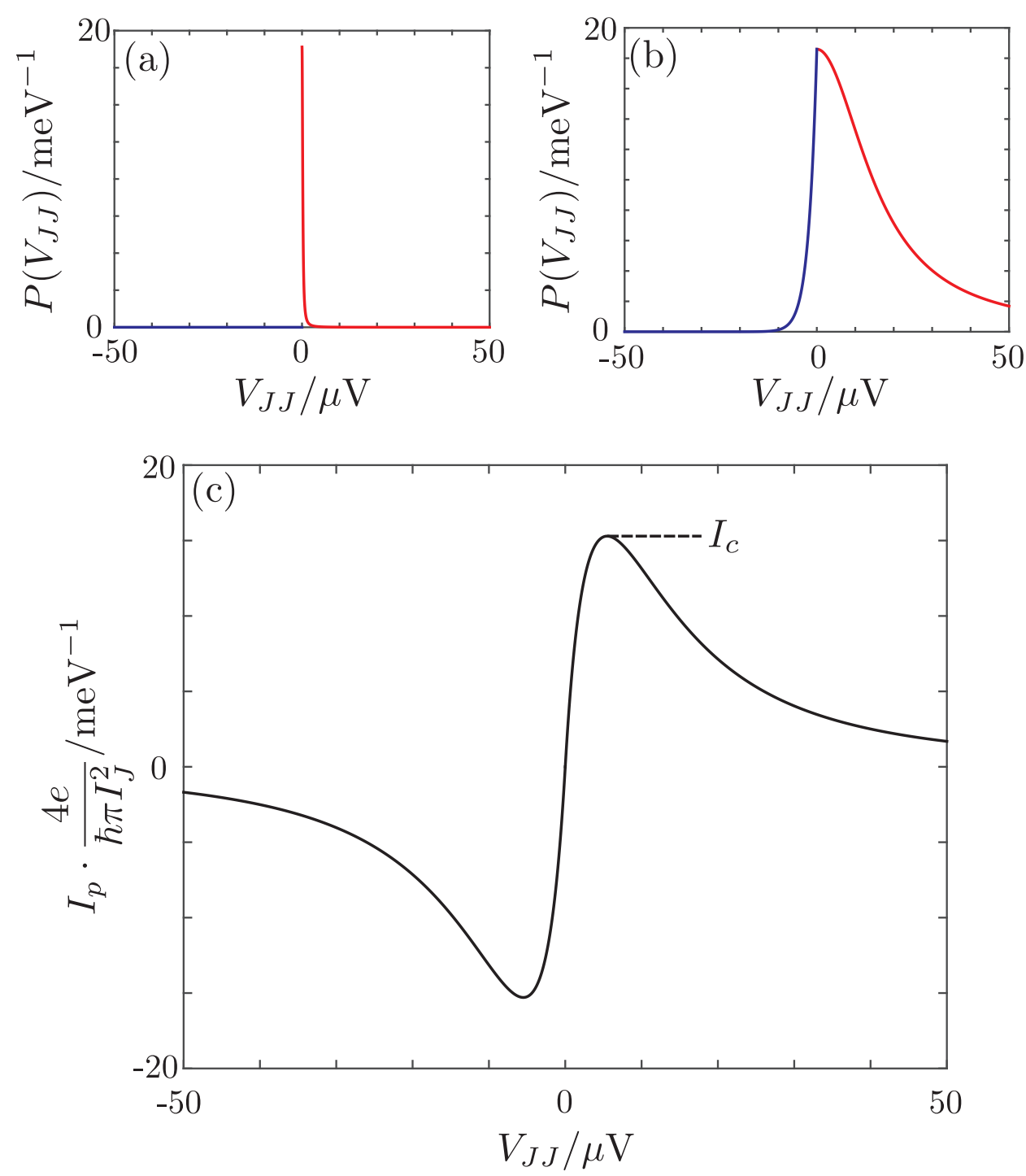

FIGURE 5.9: (a) Schematic $P(E)$ for an SJTM junction at $T=0$. The probability for a Cooper-pair to tunnel whilst emitting a photon (red) is strongly peaked at $V_{J J}=0$. The probability for tunnelling with absorption of a photon (blue) is zero. (b) Schematic $P(E)$ for an SJTM junction at $T \neq 0$. The effect of $T \neq 0$ is to broaden the distribution and populate the modes of the environment so that a Cooper-pair may absorb a photon whilst tunnelling. (c) $I\left(V_{J J}\right)$ calculated directly from the difference of the red and blue curves in (b) (see equation 5.42). It exhibits a peak current at $V_{J J} \neq 0$ whose magnitude is proportional to $I_{J}^{2}$. This peak may be used to spatially map variations in the superconducting order parameter $\Psi$. 


\subsection{Experimental Strategy for Cuprate SJTM}

The technical challenge of large field-of-view nanometre-resolution SJTM imaging is to measure, in a reasonable time (days), an array of $I_{c}$ values with $\sim 1 \%$ precision using typically $256 \times 256$ pixels. To enact this scheme you require the following:

1. As large a superconducting tip gap, $\Delta_{T}$, as possible so as to maximise $I_{J}$ and hence the observed $I_{c}$ (see equation 5.25).

2. A $d$-wave superconducting STM tip so as to maximise its Josephson coupling to the predominantly $d$-wave superconducting condensate of the cuprates.

3. An ultra-low-vibration STM that is engineered for sub-picometre stability in taking spectroscopic data while out of feedback. It should operate at the lowest practical temperatures to minimise the surpression of $I_{c}$ by thermal fluctuations.

4. Environmental radio-frequency (RF) shielding to protect the Josephson junctions formed by the tip and sample from ambient microwave radiation.

Motivated by point 1) above we achieve a large $\Delta_{T}$ by picking up a nanometre-sized flake of $\mathrm{Bi}_{2} \mathrm{Sr}_{2} \mathrm{CaCu}_{2} \mathrm{O}_{8+\delta}$ on the end of our tungsten tip. This also satisfies point 2) because the tip has a $d$-wave superconducting order parameter. Points 3) and 4) are fulfilled by using a dilution refrigerator based STM operating below $50 \mathrm{mK}$, housed in an RF shielded, vibrationally isolated room of the type described in section 2.5 .

Clearly the fabrication of $\mathrm{Bi}_{2} \mathrm{Sr}_{2} \mathrm{CaCu}_{2} \mathrm{O}_{8+\delta}$ nano-flake superconducting tips was critical to our experimental scheme. In the following section I will describe the fabrication and characterisation of these tips in greater detail.

\subsection{Superconducting STM Tips for Cuprate SJTM}

In the following $\mathrm{I}$ will discuss the fabrication of the $\mathrm{Bi}_{2} \mathrm{Sr}_{2} \mathrm{CaCu}_{2} \mathrm{O}_{8+\delta}$ nano-flake tips used in our cuprate SJTM experiments. I will also characterise the superconductivity and geometry of these tips as well as demonstrating their stability during spectroscopic imaging. 


\subsubsection{Fabrication of Superconducting $\mathrm{Bi}_{2} \mathrm{Sr}_{2} \mathrm{CaCu}_{2} \mathrm{O}_{8+\delta}$ Nano-flake Tips}

For the reasons discussed above, in implementing cuprate SJTM we chose to make superconducting tips by picking up a piece of our $\mathrm{Bi}_{2} \mathrm{Sr}_{2} \mathrm{CaCu}_{2} \mathrm{O}_{8+\delta}$ sample on the end of normal tungsten STM tips. This was achieved by manually adjusting the $z$-piezo voltage to extend the tip into the fragile $\mathrm{Bi}_{2} \mathrm{Sr}_{2} \mathrm{CaCu}_{2} \mathrm{O}_{8+\delta}$ surface. However, this does not always result in a superconducting tip.

The first crucial test of whether the tip is superconducting is to measure the differential tunnelling conductance spectrum $g\left(V_{B}\right)$. For a normal tungsten STM tip on a $\mathrm{Bi}_{2} \mathrm{Sr}_{2} \mathrm{CaCu}_{2} \mathrm{O}_{8+\delta}$ surface this is shown as the dashed curve in figure 5.10. This conductance spectrum can be thought of as the superconducting density of states in the material (see section 2.1.4). If, after picking up a piece of the sample, both tip and sample have a superconducting density of states, this converts the measured superconductor-insulatornormal (SIN) tunnelling spectrum to a superconductor-insulator-superconductor (SIS) spectrum.

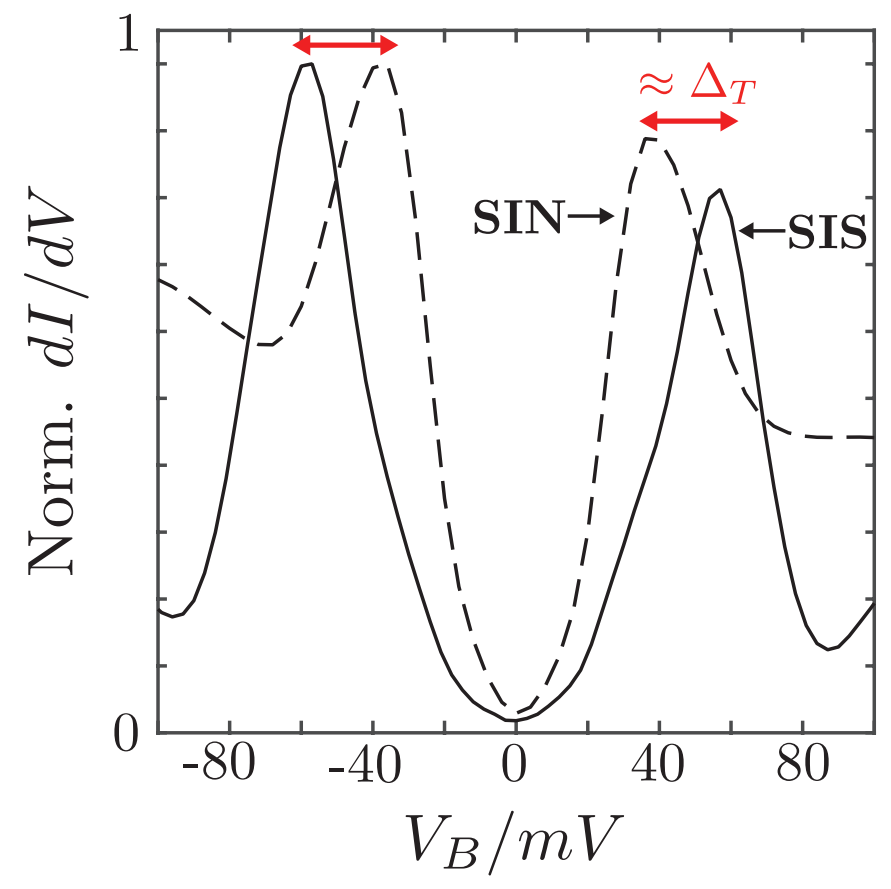

Figure 5.10: Conversion of single-particle SIN (dashed) to single-particle SIS (solid) spectra when $\mathrm{Bi}_{2} \mathrm{Sr}_{2} \mathrm{CaCu}_{2} \mathrm{O}_{8+\delta}$ nano-flake adheres to tungsten tip. The tip gap $\Delta_{T} \approx$ $25 \mathrm{meV}$ is the difference between the SIN and SIS peaks (red double-headed arrows). 
As detailed in section 2.1.4, the SIS single-particle tunnelling current, $I\left(V_{B}\right)$, is given by,

$$
I\left(V_{B}\right)=\frac{2 \pi e}{\hbar}|M|^{2} \int_{0}^{e V_{B}} n_{S}(\epsilon) n_{T}\left(E+e V_{B}\right) d \epsilon,
$$

and the differential conductance,

$$
g\left(V_{B}\right)=\frac{d I}{d V_{B}}=\frac{2 \pi e^{2}}{\hbar}|M|^{2}\left[n_{S}(E) n_{T}\left(E+e V_{B}\right)+\int_{0}^{e V_{B}} n_{S}(\epsilon) \frac{\partial}{\partial\left(e V_{B}\right)} n_{T}\left(\epsilon+e V_{B}\right) d \epsilon\right]
$$

where $n_{S}(E)$ and $n_{T}(E)$ are the sample and tip superconducting density of states respectively.

For tip and sample superconductors with single-particle coherence peaks separated by $2 \Delta_{T}$ and $2 \Delta_{S}$ respectively, this leads to an SIS spectrum with an energy separation $2 \Delta_{S I S} \approx 2\left(\Delta_{S}+\Delta_{T}\right)$ between conductance peaks. This occurs because the differential tunnelling conductance will peak when $V_{B}$ is such that the unfilled coherence peak of the tip aligns in energy with the filled coherence peak of the sample and visa versa. This is most easily understood graphically as shown in figure 5.11.

Figure 5.10 demonstrates this phenomenology for one of our $\mathrm{Bi}_{2} \mathrm{Sr}_{2} \mathrm{CaCu}_{2} \mathrm{O}_{8+\delta}$ nanoflake tips. The dashed line is a spatially averaged SIN tunnelling spectrum taken with a normal tungsten STM tip on a $\mathrm{Bi}_{2} \mathrm{Sr}_{2} \mathrm{CaCu}_{2} \mathrm{O}_{8+\delta}$ sample with hole concentration $p=0.17$. The solid line is an average SIS spectrum between a $\mathrm{Bi}_{2} \mathrm{Sr}_{2} \mathrm{CaCu}_{2} \mathrm{O}_{8+\delta}$ nanoflake tip and $\mathrm{a}_{2} \mathrm{Sr}_{2} \mathrm{CaCu}_{2} \mathrm{O}_{8+\delta}$ sample of the same hole concentration. The difference in energy between the coherence peaks of the two spectra (indicated by red double headed arrows) indicates that the tip has a gap $\Delta_{T} \approx 25 \mathrm{meV}$.

That the tunnelling spectra of our nano-flake tips show strong agreement with our expectations for SIS tunnelling is good evidence that they are indeed superconducting. Of course, the strongest evidence comes from the fact that the nano-flake tips exhibit an $I\left(V_{B}\right)$ for $V_{B} \lesssim 100 \mu \mathrm{V}$ that is characteristic of Cooper-pair tunnelling. This will be discussed in detail in section 5.6.

\subsubsection{Characterisation of Nano-flake Tip Geometry}

Having characterised the superconductivity in our nano-flake tips I will now move on to discuss their geometry, i.e their size, resolution and orientation with respect to the 


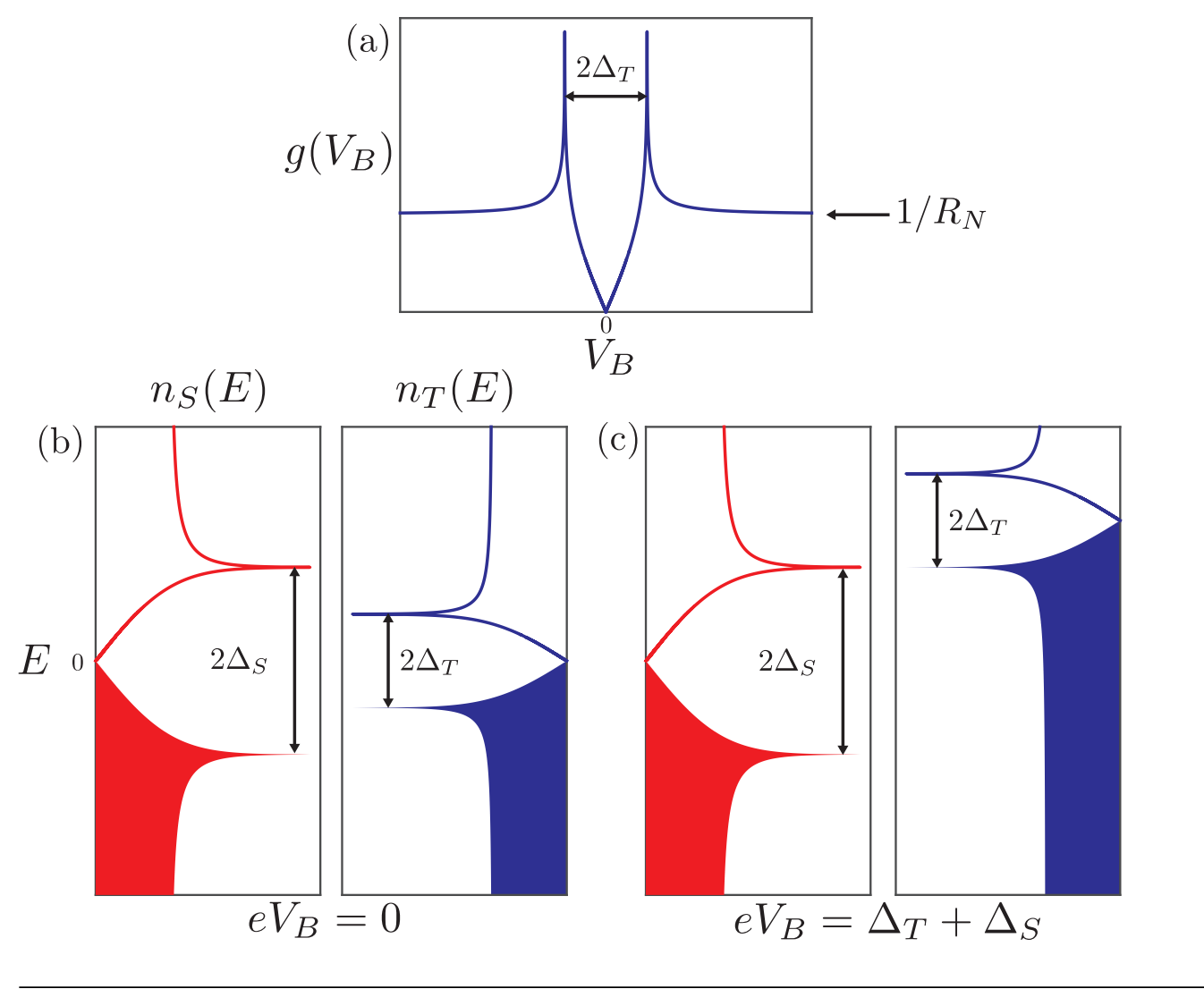

FiguRE 5.11: (a) Schematic $d I / d V_{B}$ spectrum for a $d$-wave superconductor with gap $\Delta_{T}$. (b) Schematic of SIS tunnelling between a superconducting tip with energy gap $\Delta_{T}$ and sample with gap $\Delta_{S}$. Filled states are shaded. No bias has been applied across the junction and there is no net tunnelling current. (c) A bias $\left(\Delta_{S}+\Delta_{T}\right) / e$ is applied across the junction that aligns the filled coherence peak of the tip and the empty coherence peak of the sample. This gives rise to a peak in $d I / d V_{B}$.

crystallographic axes of the sample crystal.

In figure $5.12 \mathrm{I}$ compare the performance of two completely different $\mathrm{Bi}_{2} \mathrm{Sr}_{2} \mathrm{CaCu}_{2} \mathrm{O}_{8+\delta}$ nano-flake tips. Figures 5.12 (a) and (b) show their measured $d I / d V_{B}$ spectra; they are obviously very similar. Figures 5.12 (c) and (d) show SIS topographic images for these two tips and figures 5.12 (e) and (f) are their corresponding Fourier transforms.

In both the real-space topographs and their Fourier transforms the $\mathrm{Bi}_{2} \mathrm{Sr}_{2} \mathrm{CaCu}_{2} \mathrm{O}_{8+\delta}$ bulk crystal super-modulation (see section 2.6.1) is easily resolved. Further, for both tips, topographic inhomogeneities can be resolved on the scale of $\sim 1 \mathrm{~nm}$. Together these observations indicate that our nano-flake tips have a spatial resolution of $\sim 1 \mathrm{~nm}$.

Although Bragg peaks at reciprocal lattice vectors can be seen in both Fourier transforms (figures 5.12 (e) and (f)), this does not indicate that our tips have true atomic resolution. Strong atomic modulations would occur for a tip of infinite extent, as long 

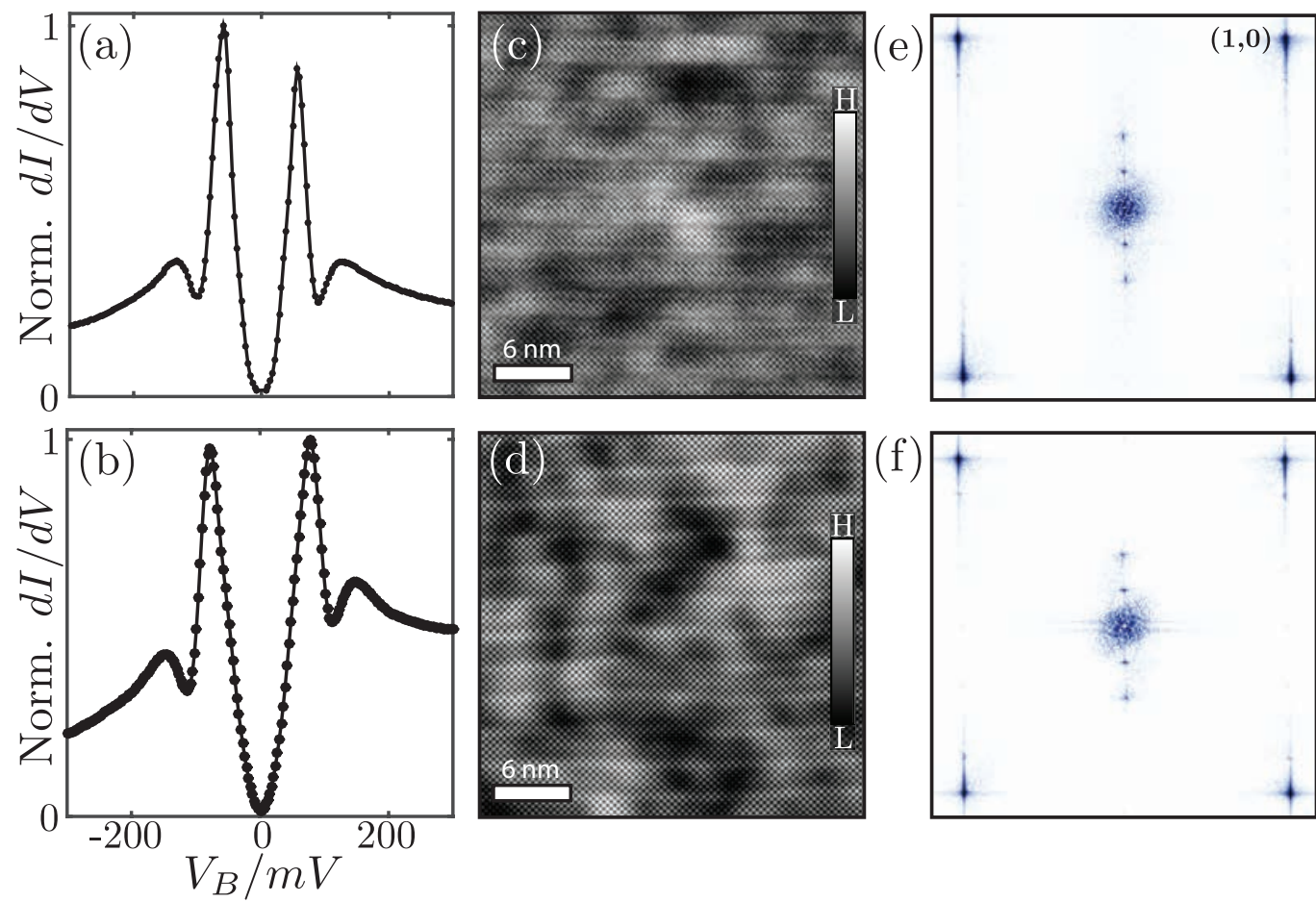

Figure 5.12: Typical $d I / d V_{B}$ spectrum, topography and magnitude of its Fourier transform $\tilde{T}(\vec{q})$ measured with two completely distinct $\mathrm{Bi}_{2} \mathrm{Sr}_{2} \mathrm{CaCu}_{2} \mathrm{O}_{8+\delta}$ tips on two different $\mathrm{Bi}_{2} \mathrm{Sr}_{2} \mathrm{CaCu}_{2} \mathrm{O}_{8+\delta}$ samples: tip 1 ((a), (c) and (e)) and tip 2 ((b), (d) and (f)).

as its crystallographic axes are aligned with the sample, due to the strong variation in tunnelling matrix elements as the atoms in the tip and sample move in and out of alignment.

To check the relative orientation of the $\mathrm{Bi}_{2} \mathrm{Sr}_{2} \mathrm{CaCu}_{2} \mathrm{O}_{8+\delta}$ nano-flake crystal axes to the axes of the sample, we evaluate simulations to see what might be expected of such a tip. Figures 5.13 (a) and (b) show a measured SIN topograph $T(\vec{r})$ of $\mathrm{Bi}_{2} \mathrm{Sr}_{2} \mathrm{CaCu}_{2} \mathrm{O}_{8+\delta}$ taken with a conventional tungsten tip, and its Fourier transform. To simulate what would be observed if the crystal axes of a $\mathrm{Bi}_{2} \mathrm{Sr}_{2} \mathrm{CaCu}_{2} \mathrm{O}_{8+\delta}$ nano-flake were oriented (or not) to those of the sample crystal axes, we extract a typical $\sim 2$-nm-diameter patch from this image and then convolute the patch image with $T(\vec{r})$. This results in a simulated SIS topograph that is expected when using a $\mathrm{Bi}_{2} \mathrm{Sr}_{2} \mathrm{CaCu}_{2} \mathrm{O}_{8+\delta}$ nano-flake tip with (or without) axes aligned to the crystal axes.

If the axes of the patch are aligned to the crystal as in figure 5.13 (c), the result is as in figure 5.13 (d). If the axes are misaligned with the crystal as in figure 5.13 (e), the result is as in figure 5.13 (f) (see the Fourier transform in the inset). As the vivid Moiré 

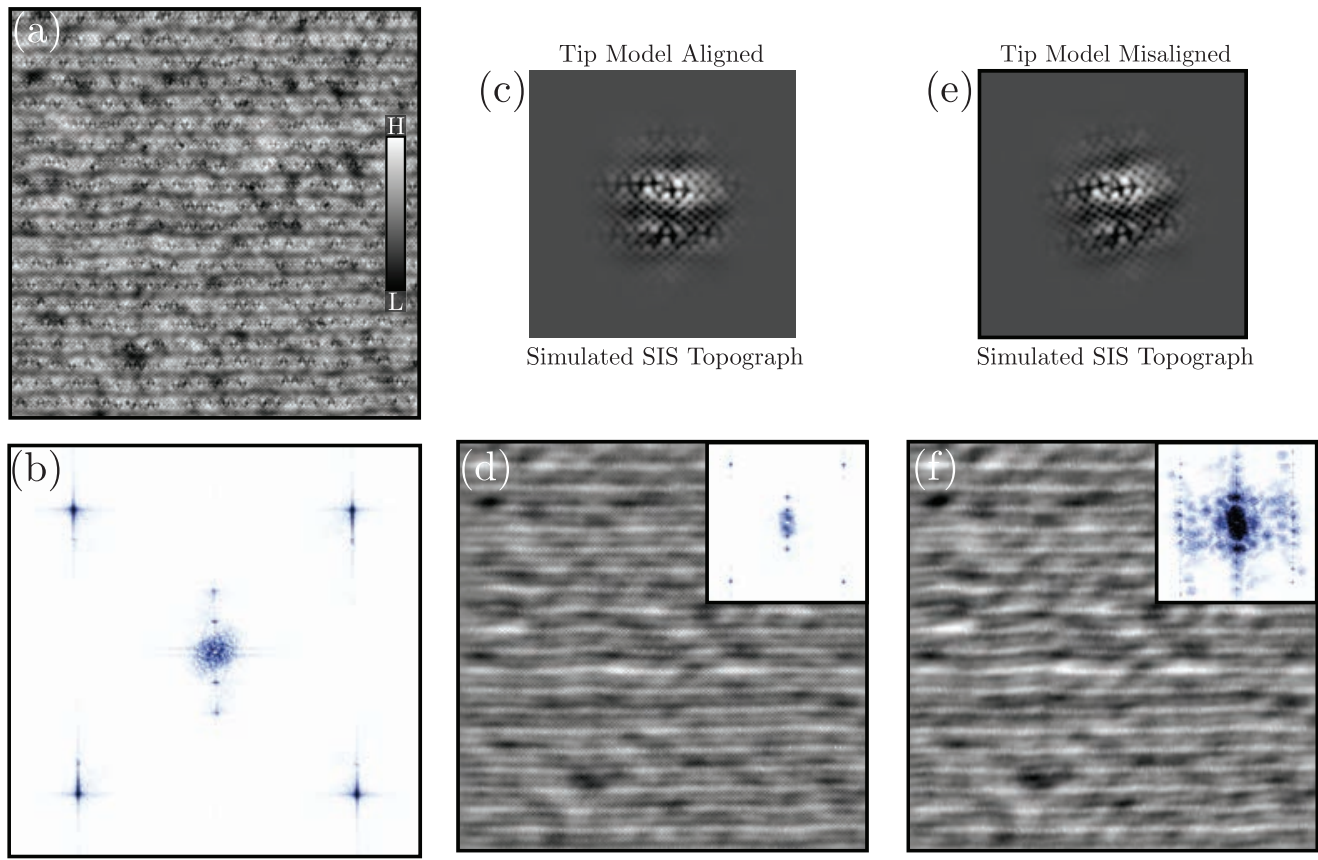

Figure 5.13: (a) Surface topography $T(\vec{r})$ of $\mathrm{Bi}_{2} \mathrm{Sr}_{2} \mathrm{CaCu}_{2} \mathrm{O}_{8+\delta}$ sample, obtained with a conventional metallic tungsten tip. (b) Magnitude of Fourier transform (FT) of (a). (c) Model for $\mathrm{Bi}_{2} \mathrm{Sr}_{2} \mathrm{CaCu}_{2} \mathrm{O}_{8+\delta}$ nanoflake tip that is aligned with the $\mathrm{Bi}_{2} \mathrm{Sr}_{2} \mathrm{CaCu}_{2} \mathrm{O}_{8+\delta}$ surface in (a). (d) Convolution of $\mathrm{Bi}_{2} \mathrm{Sr}_{2} \mathrm{CaCu}_{2} \mathrm{O}_{8+\delta}$ surface image in (a) and aligned $\mathrm{Bi}_{2} \mathrm{Sr}_{2} \mathrm{CaCu}_{2} \mathrm{O}_{8+\delta}$ nanoflake tip in (c). Inset shows resultant Fourier Transform with Bragg peaks in the corners and supermodulation peaks. (e) Model for BSCCO nanoflake tip that is misaligned with the $\mathrm{Bi}_{2} \mathrm{Sr}_{2} \mathrm{CaCu}_{2} \mathrm{O}_{8+\delta}$ surface in (a). (f) Convolution of $\mathrm{Bi}_{2} \mathrm{Sr}_{2} \mathrm{CaCu}_{2} \mathrm{O}_{8+\delta}$ surface image in (a) and misaligned $\mathrm{Bi}_{2} \mathrm{Sr}_{2} \mathrm{CaCu}_{2} \mathrm{O}_{8+\delta}$ nanoflake tip in (e). Inset shows resulting Fourier transform with many additional broad peaks caused by Moiré pattern effects. Comparison to (d) and figures 5.12 (e) and (f) demonstrates that the tip used in the studies reported here was well aligned with the sample.

effects in the Fourier transform of the latter are not observed in the actual experiments (figures 5.12 (e) and (f)), we conclude that the axes of the nano-flake are aligned with those of the $\mathrm{Bi}_{2} \mathrm{Sr}_{2} \mathrm{CaCu}_{2} \mathrm{O}_{8+\delta}$ bulk crystal. This is perhaps not surprising given that the nano-flake tips were extracted from the very same surface that they are measuring.

\subsubsection{Stability of Tips during $I_{c}(\vec{r})$ Measurement}

Given that our nano-flake tips are extended objects extracted from the fragile $\mathrm{Bi}_{2} \mathrm{Sr}_{2} \mathrm{CaCu}_{2} \mathrm{O}_{8+\delta}$ surface it is important to establish that our tips are in the vacuum tunnelling limit, as opposed to the point contact regime, and that they exhibit the stability necessary for spectroscopic imaging. The latter requires that the tip is not altered by 
interaction with the surface during measurement, so that a repeat of that measurement yields an almost identical result.

In figure 5.14 (a) and (b) we show topographic images taken with the same $\mathrm{Bi}_{2} \mathrm{Sr}_{2} \mathrm{CaCu}_{2} \mathrm{O}_{8+\delta}$ nano-flake tip before and after a typical $I_{c}(\vec{r})$ mapping experiment. Comparison of the topographs shows them to be virtually identical and demonstrates that no significant changes occur in the geometry of the tip during the map. The implication is that the nano-flake at the end of the tip never once touches the surface and that all such $I_{c}(\vec{r})$ studies reported are in the vacuum tunnelling limit.
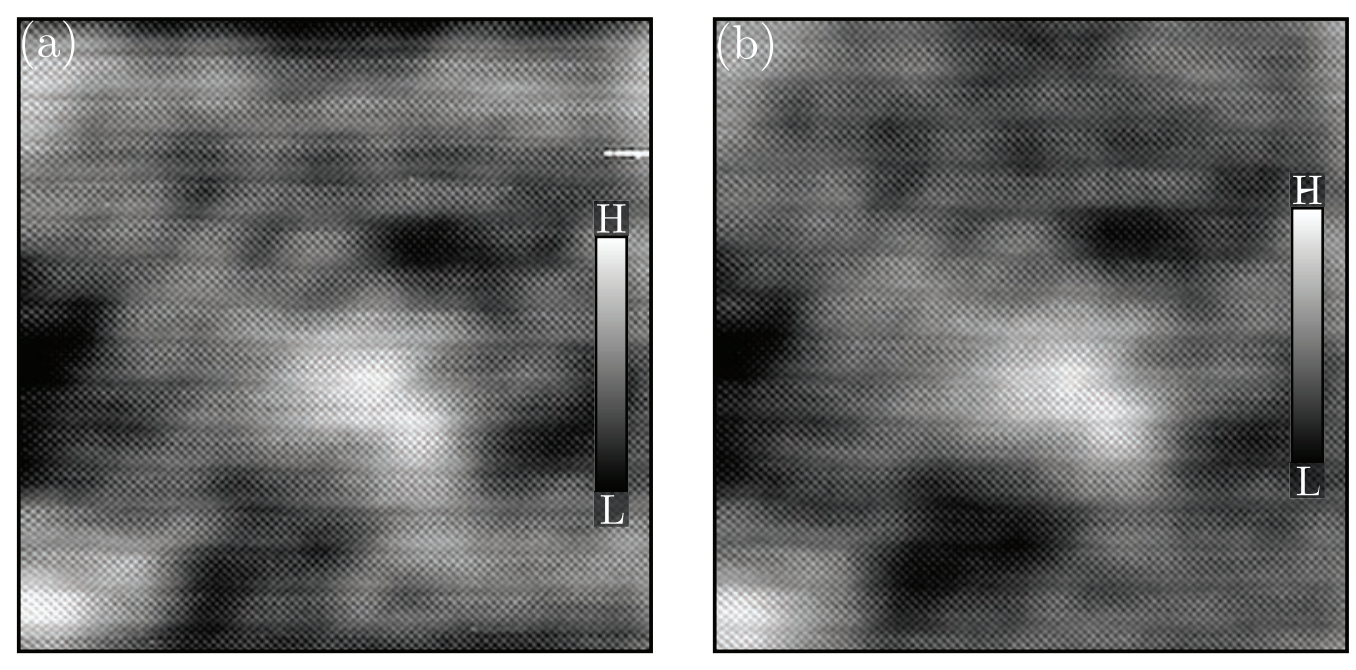

Figure 5.14: (a) Topograph taken with $\mathrm{Bi}_{2} \mathrm{Sr}_{2} \mathrm{CaCu}_{2} \mathrm{O}_{8+\delta}$ tip before typical $I_{c}(\vec{r})$ SJTM map. (b) Topograph taken with same $\mathrm{Bi}_{2} \mathrm{Sr}_{2} \mathrm{CaCu}_{2} \mathrm{O}_{8+\delta}$ tip after the same $I_{c}(\vec{r})$ SJTM map. Comparison of (a) and (b) shows that tip and surface are very well preserved in our SJTM protocol.

\subsection{Experimental Josephson Current-Voltage Characteris- tics}

In the following I will make predictions for the $I\left(V_{B}\right)$ and $I\left(V_{J J}\right)$ we expect to measure for Josephson tunnelling between a $\mathrm{Bi}_{2} \mathrm{Sr}_{2} \mathrm{CaCu}_{2} \mathrm{O}_{8+\delta}$ sample and the $\mathrm{Bi}_{2} \mathrm{Sr}_{2} \mathrm{CaCu}_{2} \mathrm{O}_{8+\delta}$ nano-flake tips characterised in the previous section. The physics of ultra-small Josephson junctions such as these was discussed in section 5.3.1. The strong non-linearity of the $I\left(V_{J J}\right)$ characteristics expected for these Josephson junctions means that the form of $I\left(V_{B}\right)$ measured in experiments is very sensitive to other elements in the measurement 
circuit. Here I will include details of the measurement circuit used in our SJTM experiments and predict $I\left(V_{B}\right)$ and $I\left(V_{J J}\right)$ that can be compared directly with experiment.

Figure 5.15 (a) shows a diagram of the hybrid STM/SJTM measurement circuit used in our experiments. A load resistor $R_{B}=10 \mathrm{M} \Omega$ is biased, in series with the Josephson junction formed between the STM tip and the sample, by a voltage $V_{B}$. The tip-sample junction is represented by the parallel combination of an ideal Josephson element with intrinsic critical current, $I_{J}$, a junction capacitance, $C_{J}$, and non-linear resistor, $R\left(V_{J J}\right)$, that represents the SIS single-particle tunnelling channel. The voltage dropped across the junction is denoted as $V_{J J}$. The ideal ammeter in this diagram is a low-noise currentto-voltage pre-amplifier in actual experiments, as detailed in figure 2.9.

To model the $I\left(V_{B}\right)$ characteristics of the circuit I will take the current due to incoherent Cooper-pair tunnelling, $I_{P}\left(V_{J J}\right)$, to be,

$$
I_{P}\left(V_{J J}\right)=a I_{J}^{2} \frac{V_{J J}}{V_{J J}^{2}+V_{C}^{2}},
$$

which is the form predicted by Ivanchenko and Zi'lberman for classical phase diffusion in the limit $C_{J} \rightarrow 0$ (see section 5.3.1) [152]. Here I use this function only as an approximate analytic form for the pair current because it exhibits a pair current peak at voltage $V_{C} \neq 0$ which scales in magnitude with $I_{J}^{2}$. As detailed in section 5.3.4 these are generic features of ultra-small Josephson junctions in a low impedance environment and so this approach is justified. Equation 5.47 is not intended to be used to extract the absolute value of $I_{J}$.

Although the Josephson junction could be regarded as current biased due to the use of a large series resistance, it can be effectively treated as voltage biased, as discussed in detail in section 5.3.2 [155, 157]. This is because the parasitic capacitance between the leads on either side of the junction, $C_{P}$, is typically much larger than the capacitance of the junction itself. As a result, the voltage across $C_{P}$ can be well approximated as independent of time even though the current flowing through the Josephson junction consists of current spikes corresponding to tunnelling events. This renders the junction effectively voltage biased. 

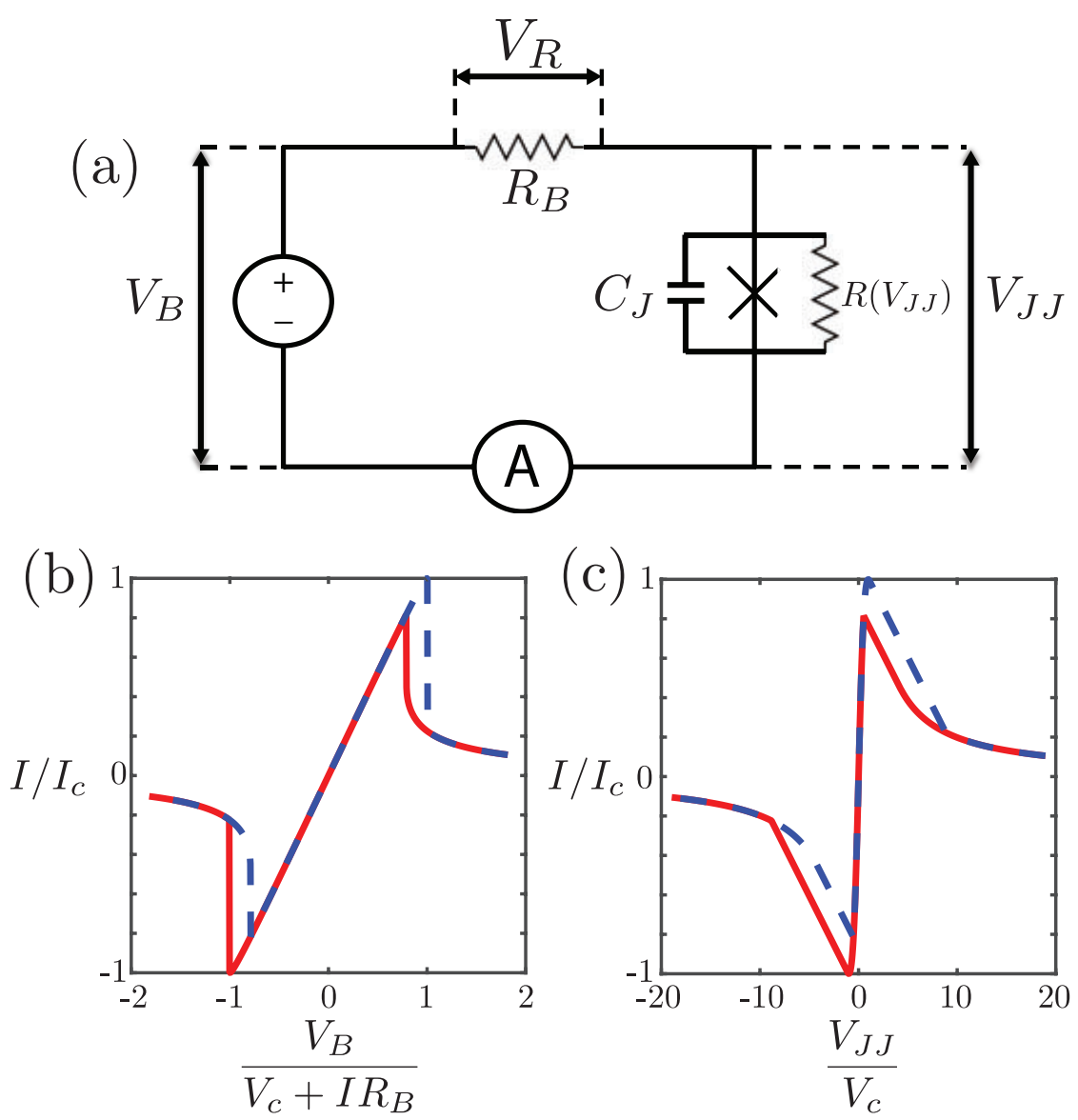

Figure 5.15: (a) Circuit diagram of the hybrid STM/ SJTM setup used in these experiments. The voltage bias $V_{B}$ is from the usual STM bias controller; the load resistor $R_{B}=10 \mathrm{M} \Omega$; the single-particle tunnelling resistance of the Josephson junction formed between the tip and sample is $R\left(V_{J J}\right)$, and the voltage actually developed across the junction is $V_{J J}$. (b) The dynamics of this circuit produces two predominant effects. First there is a discontinuous change in the $I\left(V_{B}\right)$ characteristic when the current reaches a value $I_{c} \propto I_{J}^{2}$ measured with the external ammeter shown. The second predicted effect is strong hysteresis depending on which direction the external voltage is swept; this is shown as the difference between the solid-red and dashed-blue lines. Both effects are seen very clearly and universally in the measured $I\left(V_{B}\right)$ throughout our studies. (c) The current as in (b) but plotted against the voltage across the Josephson junction $V_{J J}$.

The DC $I\left(V_{B}\right)$ for the circuit shown in figure 5.15 (a) is then given by the simultaneous solution of

$$
I=\frac{V_{R}}{R_{B}}=a I_{J}^{2} \frac{V_{J J}}{V_{J J}^{2}+V_{C}^{2}}
$$

and

$$
V_{B}=V_{R}+V_{J J}
$$

We set $R\left(V_{J J}\right)=\infty$ because the shunt provided by the SIS single-particle tunnelling channel has a characteristic resistance that is typically much larger than that of the 
Josephson junction for small $V_{J J}$.

Figure 5.15 (b) shows the numerical solutions to equations 5.48. The red and blue lines show the trajectories that the forwards and backwards sweeps take through the $I\left(V_{B}\right)$ plane. The non-linearity of the circuit introduces discontinuities in the measured $I\left(V_{B}\right)$. A discontinuity occurs at the point where the current reaches the maximum pair current that the junction can pass, $I=I_{c}$, and thus makes this current experimentally identifiable by the sharp $I\left(V_{B}\right)$ feature associated with it. Figure 5.15 (c) shows the same trajectories but as a function of the voltage across the junction $V_{J J}$.

This circuit's non-linearity also generates hysteresis in the $I\left(V_{B}\right)$ characteristic even if the dynamics of the Josephson junction itself are non-hysteretic. This hysteresis is indeed observed throughout our SJTM studies of $\mathrm{Bi}_{2} \mathrm{Sr}_{2} \mathrm{CaCu}_{2} \mathrm{O}_{8+\delta}$ and systematic errors due to the hysteresis are avoided during our $I=I_{c}(\vec{r})$ imaging experiments by sweeping the applied voltage always in the same direction once the Josephson junction has been formed at each location $\vec{r}$.

We are now in a position to compare the above predictions with the Josephson tunnelling signatures detected in our experiment. Figure 5.16 shows the $I\left(V_{J J}\right)$ characteristic between our $\mathrm{Bi}_{2} \mathrm{Sr}_{2} \mathrm{CaCu}_{2} \mathrm{O}_{8+\delta}$ nano-flake tip and a $\mathrm{Bi}_{2} \mathrm{Sr}_{2} \mathrm{CaCu}_{2} \mathrm{O}_{8+\delta}$ sample, for $T=$ $45 \mathrm{mK}$ and fixed $\vec{r}$, as a function of decreasing junction resistance, $R_{J}=V_{s} / I_{s}$, and thus decreasing tip-sample distance.

First notice that, as predicted in the previous section and with comparison to figure 5.15 (c), these $I\left(V_{J J}\right)$ curves have a sharp discontinuity at their maximum current and exhibit pronounced hysteresis. Further, as the Josephson critical current $I_{J}$ increases with diminishing distance, the magnitude of the pair current peak increases as expected. These features are in accord with our predictions for $I\left(V_{J J}\right)$ arising from incoherent Cooper pair tunnelling between a superconducting tip and sample. Taken together they further demonstrate that our $\mathrm{Bi}_{2} \mathrm{Sr}_{2} \mathrm{CaCu}_{2} \mathrm{O}_{8+\delta}$ nano-flake tip is indeed superconducting.

As discussed in section 5.3, the maximum Cooper pair current arising from incoherent Josephson tunnelling, $I_{c}$, is proportional to $I_{J}^{2}$ and $I_{J}$ is directly proportional to the amplitude of the sample superconducting order parameter $|\Psi|$. Thus, $I_{c}$ as measured from one of our $I\left(V_{J J}\right)$ curves is related to the sample's superconducting order parameter through $I_{c} \propto|\Psi|^{2} \propto \rho_{s}$, where $\rho_{s}$ is the sample superfluid stiffness. This means that 


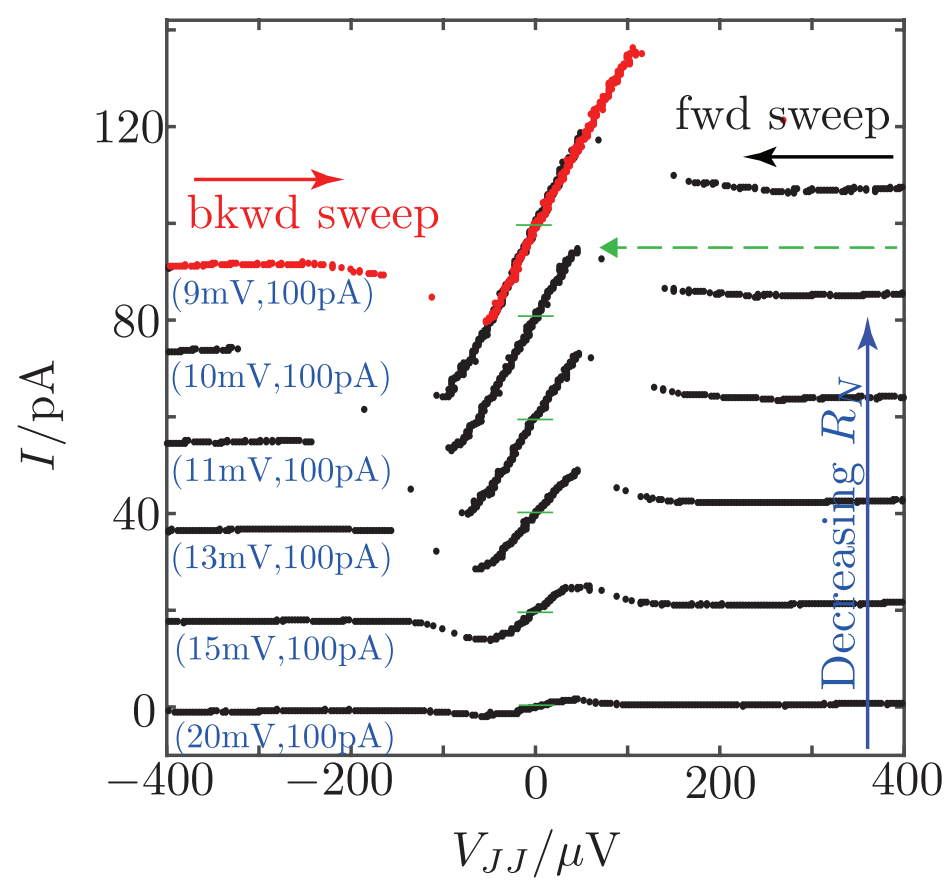

Figure 5.16: Measured evolution of the SJTM $I\left(V_{J J}\right)$ with diminishing tip-sample distance (diminishing $R_{N}$ ) at $T=45 \mathrm{mK}$ (junction formation conditions given in blue text). The maximum current $I_{c}$ for a typical $I\left(V_{J J}\right)$ used in an $I_{c}(\vec{r})$ measurement is indicated by a dashed green arrow.

by measuring $I\left(V_{J J}\right)$ at a series of points across the surface of a sample it is possible to create a spectroscopic map, $I_{c}(\vec{r})$, that directly measures spatial variations in the superconducting condensate through $I_{c}(\vec{r}) \propto \mid \Psi\left(\left.\vec{r}\right|^{2} \propto \rho_{s}(\vec{r})\right.$.

In general, we functionally define the quantity $I_{c}(\vec{r})$ to be the maximum magnitude of current achieved on sweeping away from $V_{B}=0$ with the tip at position $\vec{r}$ on the surface. An example of an $I_{c}(\vec{r})$ map is shown in figure 5.17. The most immediately apparent features are a strong quasi-periodic modulation along the vertical axis and dark regions where $I_{c}$ is almost completely suppressed. These are due to the $\mathrm{Bi}_{2} \mathrm{Sr}_{2} \mathrm{CaCu}_{2} \mathrm{O}_{8+\delta}$ bulk crystal super-modulation and individual Zn impurity atoms respectively. In the following section I will use these features to validate SJTM as a probe of the superconducting order parameter using their known effect on the superconductivity in $\mathrm{Bi}_{2} \mathrm{Sr}_{2} \mathrm{CaCu}_{2} \mathrm{O}_{8+\delta}$. 


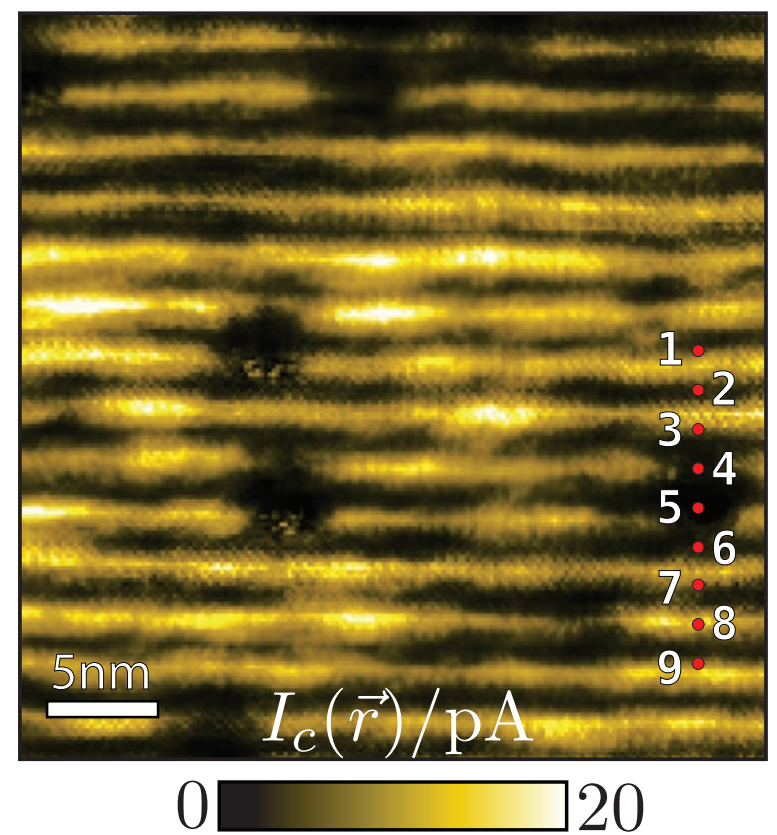

Figure 5.17: Typical SJTM $I_{c}(\vec{r})$ measurement of $\mathrm{Bi}_{2} \mathrm{Sr}_{2} \mathrm{CaCu}_{2} \mathrm{O}_{8+\delta}$ sample with $p=17 \%$ using a $\mathrm{Bi}_{2} \mathrm{Sr}_{2} \mathrm{CaCu}_{2} \mathrm{O}_{8+\delta}$ nanoflake tip. Strong quasi-periodic modulations along the vertical axis are due to the $\mathrm{Bi}_{2} \mathrm{Sr}_{2} \mathrm{CaCu}_{2} \mathrm{O}_{8+\delta}$ bulk crystal super-modulation. Red dots numbered 1-9 pass through the location of zinc impurity atom and are the locations for a series of $I\left(V_{B}\right)$ curves shown in figure 5.19 that demonstrate the almost complete suppression of $I_{c}$ at the impurity site.

\subsection{Validating SJTM as a Probe of the Order Parameter}

The experiments reported in this chapter are the first that successfully map spatial variations in the superconducting order parameter with nanometre resolution using SJTM. As such, it is pertinent to validate our SJTM method as a faithful probe of spatial variations in the superfluid density, in a model independent way. In the following we achieve this using known features of the superconductivity in $\mathrm{Bi}_{2} \mathrm{Sr}_{2} \mathrm{CaCu}_{2} \mathrm{O}_{8+\delta}$, namely a modulation of the superconducting gap with the bulk structural super-modulation and the suppression of the superfluid density at individual $\mathrm{Zn}$ impurity atoms.

\subsubsection{Imaging the Suppression of $\Psi(\vec{r})$ at Individual Zinc Impurities}

To test the Cooper-pair condensate visualisation capabilities of this $I_{c}(\vec{r})$ imaging technique directly, we use two approaches. First we study $I_{c}(\vec{r})$ in the vicinity of $\mathrm{Zn}$ impurity atoms. This is an excellent test of our technique because muon spin rotation studies 
have shown that the superfluid density is completely suppressed in a radius of a few nanometers around each Zn atom [163].

Figure 5.18 (a) shows a $76 \mathrm{~nm} \times 76 \mathrm{~nm}$ image of the SIS single-particle tunnelling conductance $g(\vec{r},-20 \mathrm{meV})$. One can locate each $\mathrm{Zn}$ impurity atom by its characteristic scattering resonance peak, which is shifted by convolution with the superconducting tip density of states from its resonance energy, $\Omega=1.5 \mathrm{meV}$, to $|E|=\Omega+\Delta_{T} \approx 25 \mathrm{meV}$ (vertical red line in the inset to figure 5.18 (a) ) [164]. Thus, the Zn impurity atoms are at the dark spots in figure 5.18 (a).

In figure 5.18 (b) I show $I_{c}(\vec{r})$ measured at $45 \mathrm{mK}$ in the same field of view as figure 5.18 (a). This reveals how $I_{c}(\vec{r})$ is suppressed within a radius of approximately $3 \mathrm{~nm}$ around each $\mathrm{Zn}$ atom site. The evolution of measured $I\left(V_{B}\right)$ curves at a specific sequence of locations along a line passing through a $\mathrm{Zn}$ impurity is shown in figure 5.19. The almost complete $(\sim 95 \%)$ suppression of $I_{c}$ as this sequence passes through the site of a $\mathrm{Zn}$ atom is seen in panel 5 of 5.19. These typical data demonstrate directly the unprocessed signal-to-noise ratio for measurement of $I_{c}$ and how the spatial evolution of unprocessed $I\left(V_{B}\right)$ spectra yields the high fidelity seen in the $I_{c}(\vec{r})$ images.

The almost complete suppression of $I_{c}(\vec{r})$ at positions which are independently determined to be $\mathrm{Zn}$ impurity sites (through their single-particle tunnelling signature) is a model independent verification that $I_{c}(\vec{r})$ measured in our SJTM experiments is a faithful probe of variations in the superconducting order parameter.

\subsubsection{Modulation of Superconductivity by the Bulk Structural Super- Modulation}

A second test is possible because of the bulk crystal super-modulation at wave-vector $\vec{Q}_{S M}$ present in $\mathrm{Bi}_{2} \mathrm{Sr}_{2} \mathrm{CaCu}_{2} \mathrm{O}_{8+\delta}$ (see section 2.6.1). One way in which this is manifest is the strong quasi-periodic modulations in the topograph $T(\vec{r})$, as shown using an normal tungsten tip in figure 5.20 (a). This super-modulation is known to modulate the single-particle tunnelling gap at the same wave-vector, as demonstrated by SIN tunnelling measurements shown in figures 5.20 (c) - (f)[88]. We searched for a concomitant modulation in $I_{c}(\vec{r})$ to further validate our cuprate SJTM method. 
(a)

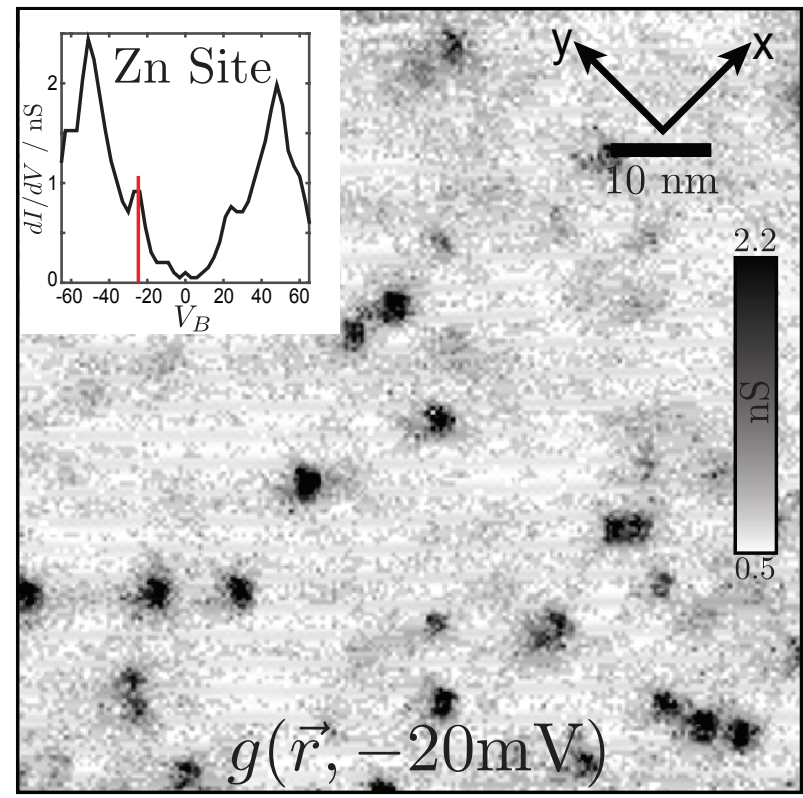

(b)

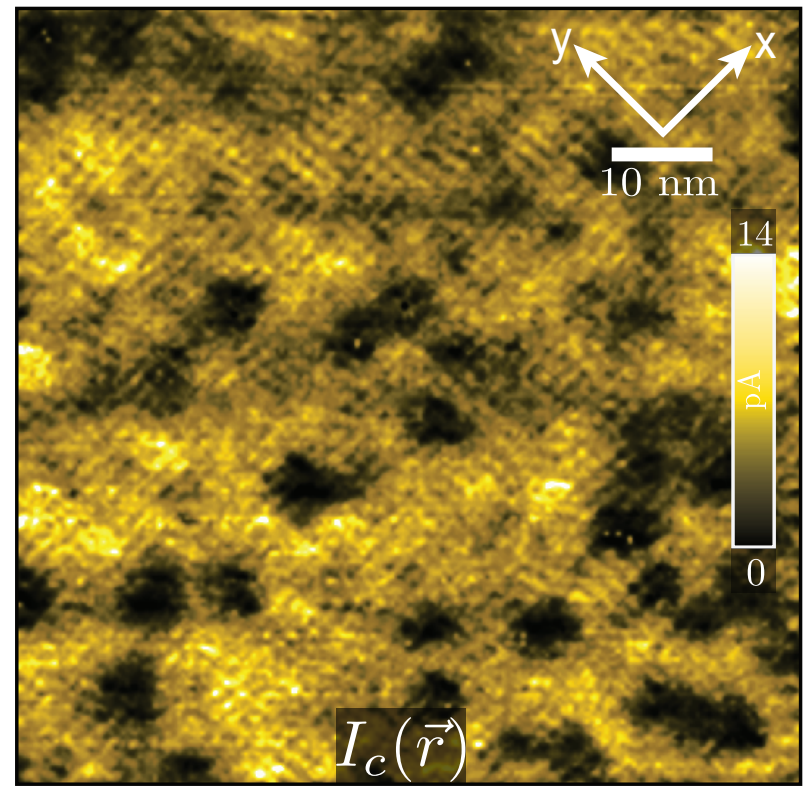

Figure 5.18: (a) Single-particle SIS conductance map $g(r, E=-20 \mathrm{meV})$. The inset is the conductance spectrum at a Zinc impurity site. The convolution of $\Omega=1.5 \mathrm{meV}$ Zn impurity resonance with the superconducting spectrum of the tip shifts the resonance to $|E| \approx|\Omega|+\left|\Delta_{T}\right|$. The measured SIS $g(r, E=-20 \mathrm{meV})$ image near this energy (vertical red line) identifies the site of each $\mathrm{Zn}$. (b) $I_{c}(\vec{r})$ image measured at $45 \mathrm{mK}$ in the same field of view as (a). A deep minimum $(\approx 95 \%$ suppression $)$ occurs in $I_{c}(\vec{r})$ surrounding each Zn site where the Cooper-pair condensate is suppressed. For visual clarity modulations at the wave-vector of the crystal super-modulation have been removed by the Fourier filtration method described in section 2.8 . 


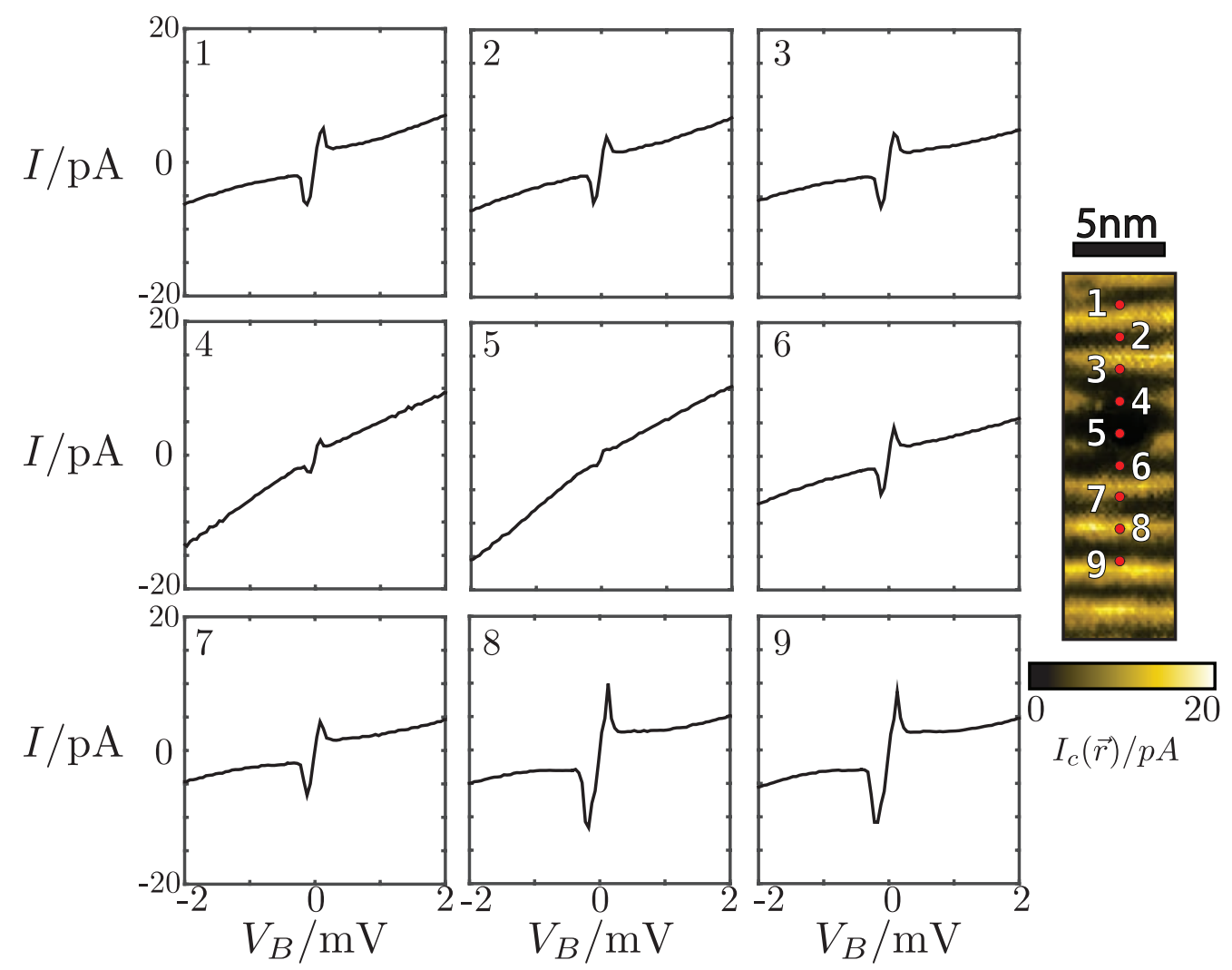

Figure 5.19: Nine individual $I\left(V_{B}\right)$ spectra measured at the locations passing through a zinc impurty site, indicated by numbers 1-9 in the $I_{c}(\vec{r})$ image (right hand side). The $I_{c}(\vec{r})$ image is a small section of that shown in figure 5.17. As the sequence passes through the site of a $\mathrm{Zn}$ atom, the value of $I_{c}$ diminishes by $\approx 95 \%$ from its maximum, as expected from $\mu \mathrm{SR}$ experiments.

Turning back to measurements made with $\mathrm{Bi}_{2} \mathrm{Sr}_{2} \mathrm{CaCu}_{2} \mathrm{O}_{8+\delta}$ nano-flake tips, figure 5.21 (a) shows clear modulations in $I_{c}$ at $\vec{Q}_{S M}$. In figure 5.21 (b) we plot the Fourier transform of the SIS topograph, $\tilde{T}(\vec{q})$, in grey and $\tilde{I}_{c}(\vec{q})$ in orange, both measured simultaneously along the $(1,1)$ direction through $\vec{Q}_{S M}$. This directly demonstrates the capability of SJTM to visualise Cooper-pair density modulations induced by the $\mathrm{Bi}_{2} \mathrm{Sr}_{2} \mathrm{CaCu}_{2} \mathrm{O}_{8+\delta}$ crystal super-modulation.

Overall, these tests show that $I_{c}(\vec{r})$ faithfully measures spatial variations in the superconducting order parameter. Thus, we have achieved nanometer resolution Cooper pair condensate visualisation using our $\mathrm{Bi}_{2} \mathrm{Sr}_{2} \mathrm{CaCu}_{2} \mathrm{O}_{8+\delta}$ nano-flake tip SJTM approach. 

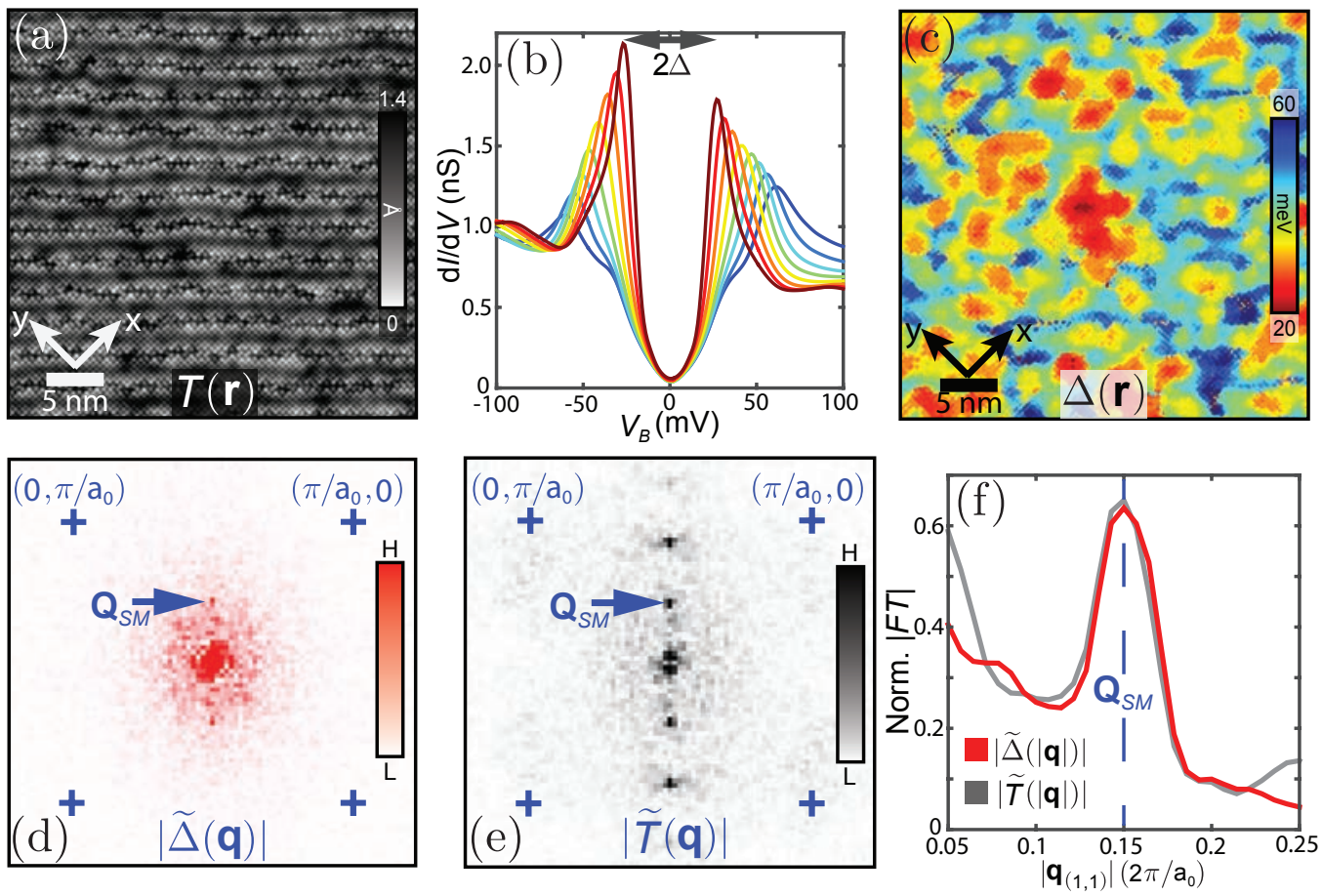

Figure 5.20: (a) Typical $35 \mathrm{~nm} \times 35 \mathrm{~nm}$ topographic image $T(\vec{r})$ of $\mathrm{Bi}_{2} \mathrm{Sr}_{2} \mathrm{CaCu}_{2} \mathrm{O}_{8+\delta}$ surface (crystal supermodulation runs vertically). (b) Typical $g(E)=d I / d V_{B}(E=\mathrm{eV})$ differential conductance spectra of $\mathrm{Bi}_{2} \mathrm{Sr}_{2} \mathrm{CaCu}_{2} \mathrm{O}_{8+\delta}$. The maximum energy gap $\Delta$ is half the distance between peaks. (c) $\Delta(\vec{r})$ (gapmap) for $p \sim 17 \% \mathrm{Bi}_{2} \mathrm{Sr}_{2} \mathrm{CaCu}_{2} \mathrm{O}_{8+\delta}$ in same field of view as (a). (d) Magnitude of Fourier transform of (c), $|\tilde{\Delta}(\vec{q})|$ (crosses at $\left.q=\left(\pi / \mathrm{a}_{0}, 0\right) ;\left(0, \pi / \mathrm{a}_{0}\right)\right)$ showing a single peak due to the super-modulation (blue arrow). (e) Fourier transform magnitude of topograph $|\tilde{T}(\vec{q})|$ (crosses at $q=$ $\left.\left(\pi / \mathrm{a}_{0}, 0\right) ;\left(0, \pi / \mathrm{a}_{0}\right)\right)$. The super-modulation is a quasi-periodic modulation along the $(1,1)$ direction with wavevector $\vec{Q}_{S M}$ indicated by blue arrow. (f) Simultaneously measured $|\tilde{\Delta}(\vec{q})|$ and $|\tilde{T}(\vec{q})|$ from (d),(e) along the $(1,1)$ direction. Their primary peaks coincide.

\subsection{Conclusions and Future Directions of SJTM}

Using a $\mathrm{Bi}_{2} \mathrm{Sr}_{2} \mathrm{CaCu}_{2} \mathrm{O}_{8+\delta}$ nanoflake tip SJTM we have made the first direct, nanometerresolution visualisation of the condensate in any superconductor. In doing so we have visualised the suppression of the superconducting order parameter at Zn impurities in $\mathrm{Bi}_{2} \mathrm{Sr}_{2} \mathrm{CaCu}_{2} \mathrm{O}_{8+\delta}$, confirming a well known result of $\mu \mathrm{SR}$ studies and validating our method.

\subsubsection{Future State of the Art in Condensate Imaging}

In detecting atomic-scale spatial variations in the superconducting condensate directly, our SJTM has fulfilled much of the promise it showed in its conception [133]. The 

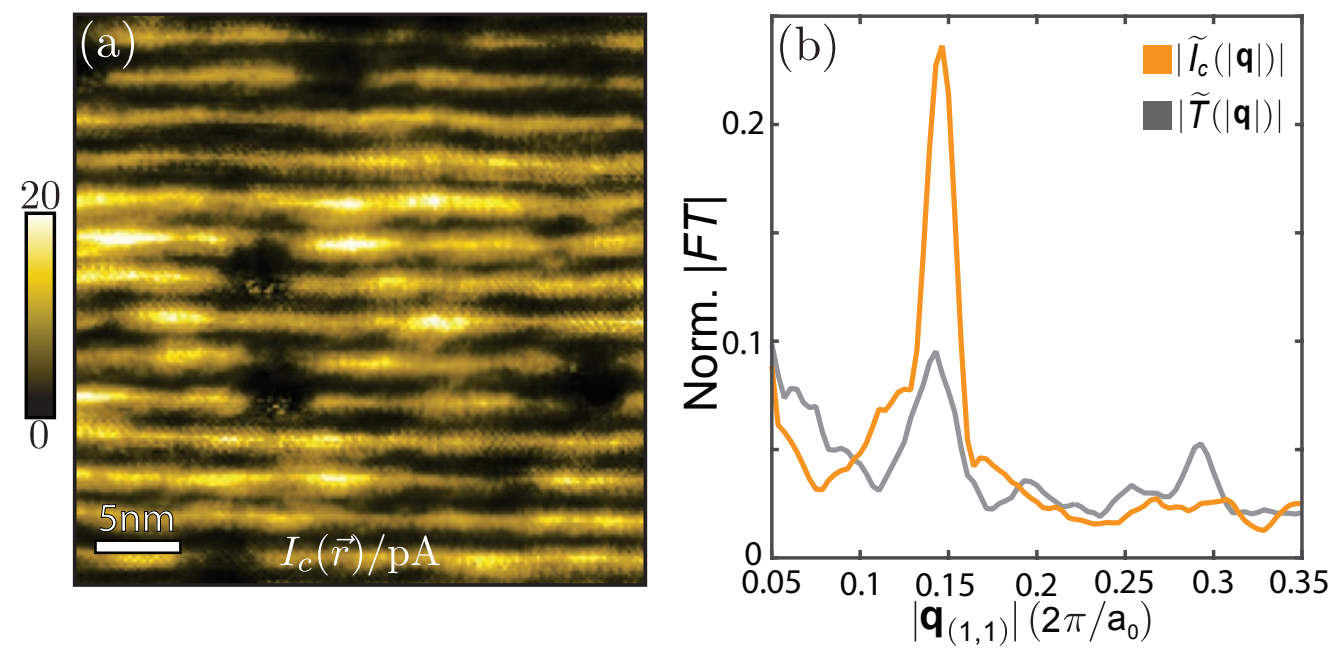

FiguRE 5.21: (a) $I_{c}(\vec{r})$ map showing clear modulations at $\vec{Q}_{S M}$. (b) Simultaneously measured magnitude of $\tilde{I}_{c}(\vec{q})$ (Fourier transform of (a)) and $\tilde{T}(\vec{q})$ along the $\mathrm{CuO}_{2}(1,1)$ direction. The primary peaks in $\tilde{I}_{c}(\vec{q})$ and $\tilde{T}(\vec{q})$ from the super-modulation coincide, demonstrating the ability of SJTM to visualise modulations in $\Psi(\vec{r})$.

next advance in the state of the art will be to achieve intra unit cell resolution imaging capabilities on a cuprate superconductor. Such resolution has recently been achieved on the surface of lead, using a lead coated tungsten tip [145]. For cuprate SJTM however, our nanoflake tip approach is unlikely to ever yield intra unit cell resolution.

An obvious scheme to improve the resolution of cuprate SJTM would be to use a superconducting tip material that can be formed into a sharp point either mechanically or by chemical/physical etching. However, these materials typically have a superconducting gap that is at least an order of magnitude smaller than a $\mathrm{Bi}_{2} \mathrm{Sr}_{2} \mathrm{CaCu}_{2} \mathrm{O}_{8+\delta}$ nanoflake tip, greatly reducing $I_{c}$ for a given junction resistance.

Furthermore, because the practical material choices are $s$-wave superconductors we expect a strong suppression of $I_{c}$ (at a given junction resistance) due to the fact that the $s$-wave component, $\Delta_{s}$ of the cuprate order parameter ranges from $\Delta_{s} \approx 0-0.1 \Delta_{d}$, depending on cuprate material $[165,166]$. To achieve a similar magnitude of $I_{c}$ as those reported here, the junction resistance would have to be greatly reduced.

The micaceous nature of $\mathrm{Bi}_{2} \mathrm{Sr}_{2} \mathrm{CaCu}_{2} \mathrm{O}_{8+\delta}$ makes its surface unstable to a sharp metallic tip at low junction resistances. However, $\mathrm{YBa}_{2} \mathrm{Cu}_{3} \mathrm{O}_{6+x}$ has $\Delta_{s} \sim 0.1 \Delta_{d}$ and a stable surface. This material, in combination with a conventional superconducting STM tip, could yield intra unit cell resolution cuprate SJTM. 


\subsubsection{Inelastic Cooper-pair Tunnelling Spectroscopy}

SJTM utilises the inelastic tunnelling of Cooper-pairs to access the superconducting order parameter. As discussed in section 5.3.3, this is the simplest form of inelastic tunnelling because the Cooper-pair condensate does not form a continuum of initial and final states. This allows an easier route to determine the environmental impedance, $Z_{t}(\omega)$, than inelastic single-particle tunnelling spectroscopy.

While the super-current peak observed in our SJTM $I\left(V_{J J}\right)$ is a generic result of the small ohmic impedance provided by the environment, in principle we can detect resonances in $Z_{t}(\omega)$. Indeed, resonances arising from the antenna modes of the tip have already been observed in the Josephson tunnelling current [142, 143, 145].

If a molecule absorbed onto a superconducting substrate has an absorption line at a frequency $\nu$, it could be detected in the inelastic Cooper-pair tunnelling current as a peak at $2 e V_{J J}=h \nu$. Alternatively the $\mathrm{GHz}$ radiation field emitted from a typical SJTM junction could be used in the coherent manipulation of single spins as suggested in reference [143], where large GHz photon fluxes have already been demonstrated. The principle advantage of performing atomically resolved spectroscopy or atomic manipulation of this kind with SJTM as opposed to a normal STM tip, is that the source and detector of high frequency signals is the junction itself. As such, normal low-temperature STM cryostats with wiring designed for low frequency signals can be used, greatly simplifying the experimental design. 



\section{Chapter 6}

\section{Pair Density Waves in Cuprates}

In this chapter I will define what is meant by a pair density wave (PDW). I will also discuss how the PDW couples to charge density wave, spin density wave and uniform superconducting orders. After reviewing the experimental evidence and theoretical proposals for a PDW in cuprate superconductors, I will discuss how to experimentally distinguish between these proposals.

\subsection{The Pair Density Wave}

In 1964 Fulde, Ferrel, Larkin and Ovchinnikov (FFLO) predicted a new state of matter : a superconductor where the pairing amplitude modulates periodically in space [61, 62]. The family of states with such a periodically modulating pairing amplitude are known as pair density waves [167]. Phenomenologically we can examine these states by expanding the pairing amplitude in order parameters,

$$
\begin{aligned}
& \left\langle\hat{\psi}_{\sigma}^{\dagger}\left(\vec{r}_{1}\right) \hat{\psi}_{-\sigma}^{\dagger}\left(\vec{r}_{2}\right)\right\rangle=F_{0}\left(\vec{r}_{1}-\vec{r}_{2}\right) \Psi_{0}(\vec{R}) \\
+ & F_{1}\left(\vec{r}_{1}-\vec{r}_{2}\right)\left[\Psi_{\vec{Q}_{x}}(\vec{R}) e^{i \vec{Q}_{x} \cdot \vec{R}}+\Psi_{-\vec{Q}_{x}}(\vec{R}) e^{-i \vec{Q}_{x} \cdot \vec{R}}+\Psi_{\vec{Q}_{y}}(\vec{R}) e^{i \vec{Q}_{y} \cdot \vec{R}}+\Psi_{-\vec{Q}_{y}}(\vec{R}) e^{-i \vec{Q}_{y} \cdot \vec{R}}\right]+\ldots,
\end{aligned}
$$

where $\hat{\psi}_{\sigma}^{\dagger}\left(\vec{r}_{1}\right)$ creates a quasi-particle of spin $\sigma$ at location $\vec{r}_{1}$ and $\vec{R}=\left(\vec{r}_{1}+\vec{r}_{2}\right) / 2 . \Psi_{0}$ is a complex scalar field corresponding to a uniform charge 2e superconductor. $\Psi_{\vec{Q}_{x}}, \Psi_{-\vec{Q}_{x}}$, 
$\Psi_{\vec{Q}_{y}}$ and $\Psi_{-\vec{Q}_{y}}$ are pair density wave (PDW) order parameters $[63,167]$. They also are complex scalar fields, but they carry momenta $\vec{Q}_{ \pm x}$ and $\vec{Q}_{ \pm y}$ running along orthogonal directions $\vec{x}$ and $\vec{y}$.

With the uniform superconducting component $\Psi_{0}$ equal to zero, the PDW will itself break a global U(1) phase symmetry making the pair density wave a superconductor, capable of exhibiting flux quantisation and a Meissner effect. It will also breaks lattice translational symmetry and may additionally break lattice rotational and time-reversal symmetry. In general $\Psi_{\vec{Q}}$ and $\Psi_{-\vec{Q}}$ are independent complex numbers so as to encode both the broken global $\mathrm{U}(1)$ phase symmetry that makes it a superconductor and the broken $\mathrm{U}(1)$ symmetry that corresponds to the phase of the spatial modulation.

Here I have chosen to consider a tetragonal system with quasi two-dimensional order so that the PDW wave-vectors lie in the square planes of the tetragonal lattice. In the context of the cuprate high-temperature superconductors $\vec{x}$ and $\vec{y}$ will be the two symmetry equivalent $\mathrm{Cu}-\mathrm{O}$ directions in the $\mathrm{CuO}_{2}$ planes. The symmetry of the uniform superconducting order parameter $\Psi_{0}$ is manifest in how $F_{0}\left(\vec{r}_{1}-\vec{r}_{2}\right)$ transforms under a $90^{\circ}$ rotation of the co-ordinate system. An $\mathrm{A}_{1 g}$ (s-wave) order parameter is invariant under such a rotation whereas a $\mathrm{B}_{1 g}\left(\mathrm{~d}_{x^{2}-y^{2}}\right)$ order parameter will change sign.

The function $F_{1}\left(\vec{r}_{1}-\vec{r}_{2}\right)$ is the form factor of the PDW. As for the case of the CDW discussed in chapters 1 and 4, the fact that the PDW breaks translational symmetry means that its form factor is not sufficient in itself to determine which irreducible representation of the point group the PDW transforms as. However, if $F_{1}\left(\vec{r}_{1}-\vec{r}_{2}\right)$ is even under $90^{\circ}$ rotation then the PDW can be termed to have an $s$-wave symmetry form factor whereas if it is odd it has a $d$-wave symmetry form factor [99].

The expansion in equation 6.1 can be regarded as the wave-function of a Cooper-pair and thus the coordinate $\vec{R}$ as the centre of mass of the Cooper-pair. For the uniform superconducting component, $\Psi_{0}$, the conjugate momentum, $\vec{Q}$, of the Cooper-pair is zero, as we would expect from the pairing of quasi-particles at opposite momenta in BCS theory. In the PDW state the order parameters have non-zero momentum $\pm \vec{Q}_{x, y}$ associated with them because they arise from the pairing of quasi-particles at momenta $\vec{k}$ and $-\vec{k}+\vec{Q}$ to form a Cooper-pair with centre of mass momentum $\vec{Q}$. This is shown schematically in figure 6.1 (a). 

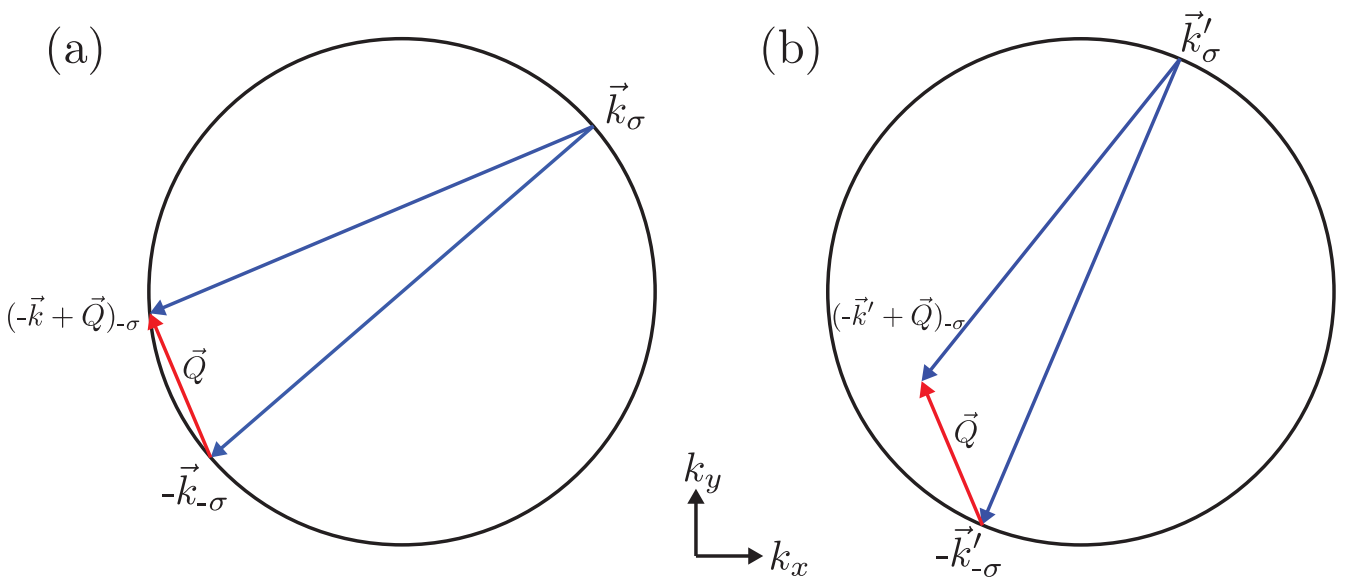

Figure 6.1: (a) Schematic of Cooper pairing on a cylindrical Fermi surface, leading to the formation of PDW state. A quasi-particle with momentum $\vec{k}$ and spin $\sigma$ pairs with another at momentum $-\vec{k}+\vec{Q}$ and spin $-\sigma$. They form a Cooper pair with centre of mass momentum $\vec{Q}$ and hence a PDW with wave-vector $\vec{Q}$. In general, there is no weak-coupling instability to the PDW state because $\vec{k}$ and $-\vec{k}+\vec{Q}$ only lie on the Fermi surface for one choice of $\vec{k}$ as depicted in (b).

In chapter $1 \mathrm{I}$ discussed how for arbitrarily weak effective interaction $U<0$, the RPA particle-particle susceptibility

$$
\chi_{p p}(\vec{q}, \omega)=\frac{\chi_{p p}^{0}(\vec{q}, \omega)}{1+U \chi_{p p}^{0}(\vec{q}, \omega)},
$$

always diverges for finite temperature for $\vec{q}, \omega \rightarrow 0$. This is because, in the presence of time-reversal symmetry, the bare susceptibility $\chi_{p p}^{0}$ diverges logarithmically with decreasing temperature, as discussed in chapter 1. This famous "BCS log" leads to uniform superconducting phases such as that represented by $\Psi_{0}$. For finite momentum pairing there is, in general, no divergent susceptibility to particle-particle pairing based on fermiology alone, as was also the case for the particle-hole (CDW) susceptibility in dimension higher than one [168]. This is apparent in figure 6.1 (b) from the fact that $\vec{k}^{\prime}=-\vec{k}+\vec{Q}$ only lies on the Fermi surface for one choice of $\vec{k}$. Therefore we expect a phase transition to a PDW state only in the presence of a special Fermi surface or strong interactions between quasi-particles.

FFLO considered an example of a special Fermi surface, i.e that of a metal in a large applied magnetic field [62]. Here, Fermi surfaces of opposite spin will be Zeeman split by an energy $g \mu_{B} B$, where $g$ is the effective g-factor for quasi-particles in the normal metal (fig. 6.2). If the material were to form a uniform superconductor, where the formation 
of spin-singlet Cooper pairs does not allow any magnetisation of the itinerant quasiparticles, the paramagnetic energy of the superconductor would be higher than that of the normal metal by $E_{p}=1 / 2 \chi_{n} H^{2}$ where $\chi_{n}$ is the spin-susceptibility of the normal metal and $H$ the magnetic induction. However, the energy of the superconductor will be lowered from that of the normal metal by its condensation energy $E_{c o n}=1 / 2 N(0) \Delta_{0}^{2}$ [8], where $\Delta_{0}$ is the BCS gap.

Somewhat rarely, it will be the competition between the energy scales $E_{p}$ and $E_{\text {con }}$ that determines the upper critical field $H_{c 2}$ of a type-II superconductor. If this were the only contribution to pair-breaking then at $T=0$ we would expect a first-order phase transition between the superconducting and normal metal states when $E_{p}=E_{c o n}$, leading to a pauli-limited upper critical field $H_{c 2}^{p}=\sqrt{2} \Delta_{0} / g \mu_{B}[169,170]$. The more usual situation is that $H_{c 2}$ is dominated by the orbital contribution; that is the decrease in condensation energy caused by the kinetic energy of the super-currents surrounding the vortex cores. In the case where the upper critical field is determined only by this orbital contribution, the upper critical field $H_{c 2}^{\text {orb }}=\Phi_{0} / 2 \pi \xi^{2}$ is reached when vortex cores overlap [171]. The relative importance of these two contributions can be measured by the Maki parameter [171]

$$
\alpha=\sqrt{2} \frac{H_{c 2}^{o r b}}{H_{c 2}^{p}} .
$$

Fulde and Ferrel noted that for materials with $\alpha \ll 1$ superconductivity can be extended to magnetic fields larger than $H_{c 2}^{p}$ by forming a pair density wave state,

$$
\left\langle\psi_{\sigma}^{\dagger}\left(\vec{r}_{1}\right) \psi_{-\sigma}^{\dagger}\left(\vec{r}_{2}\right)\right\rangle=\Psi_{\vec{Q}_{H}} e^{i \vec{Q}_{H} \cdot \vec{R}}
$$

where pairing between sections of the Zeeman split Fermi surfaces results in Cooper-pairs with centre-of-mass momentum $\vec{Q}_{H} \approx 2 \mu_{B} \vec{H} / \hbar v_{F}$ [62]. This is shown schematically in figure 6.2 (a). In this Fulde-Ferrel (FF) state the magnitude of the superconducting order parameter is constant, while its phase rotates with period $2 \pi /\left|\vec{Q}_{H}\right|$. As shown in figure $6.2(\mathrm{~b})$, only part of the Fermi surface is paired in this state with the other de-paired sections allowing a net magnetisation. While the condensation energy of the FF state is less than that of the uniform BCS state, this net magnetisation reduces the 
Zeeman energy and leads the FF state to be favoured over the uniform BCS state at high fields.

(a)

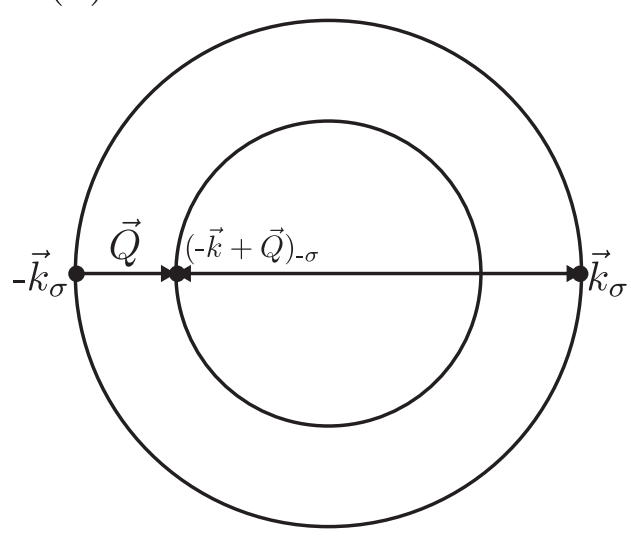

(b)

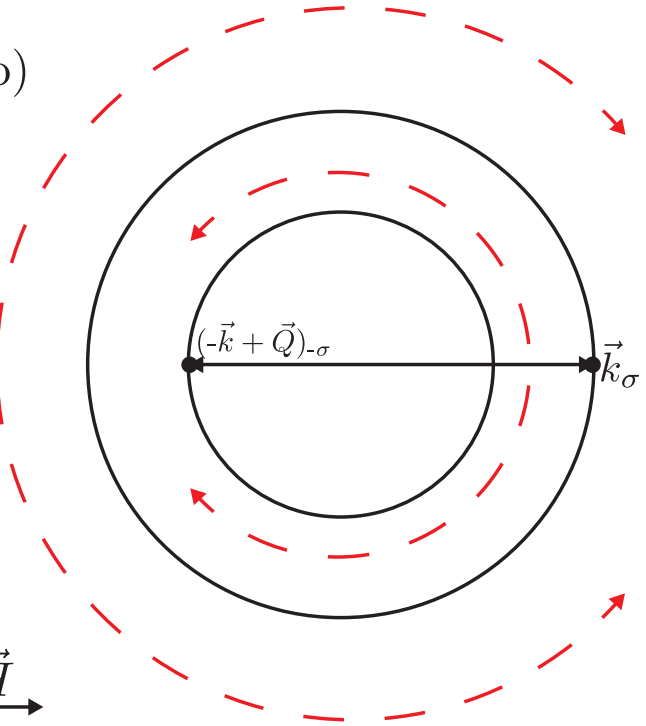

Figure 6.2: (a) Spin singlet Cooper pairing between Zeeman split Fermi surfaces leads to the formation of an FF state with wave-vector $\vec{Q}_{H} \approx 2 \mu_{B} \vec{H} / \hbar v_{F}$. (b) In such an FF state pairing only occurs on sections of the Fermi surface near the black dots, leaving large sections of Fermi surface un-paired as indicated by the dashed red lines. This allows for a net magnetisation and renders the condensation energy of the FF state less than that of the uniform BCS state.

The FF state breaks time-reversal symmetry by including a modulation of the pairing amplitude at $\overrightarrow{Q_{H}}$ but not $-\overrightarrow{Q_{H}}$. Larkin and Ovchinnikov considered a state that preserves time-reversal symmetry by taking a linear combination of these two degenerate states to give

$$
\left\langle\hat{\psi}_{\sigma}^{\dagger}\left(\vec{r}_{1}\right) \hat{\psi}_{-\sigma}^{\dagger}\left(\vec{r}_{2}\right)\right\rangle=\Psi_{\vec{Q}_{H}} e^{i \vec{Q}_{H} \cdot \vec{R}}+\Psi_{-\vec{Q}_{H}} e^{-i \vec{Q}_{H} \cdot \vec{R}}
$$

with $\Psi_{-\vec{Q}_{\vec{H}}}=\Psi *_{\vec{Q}_{\vec{H}}}[61]$. This state has a lower free-energy than the FF state (eqn. 6.4) [170]. In contrast to the FF state, in the LO state the density of Cooper-pairs is modulated.

Despite decades of searching there is still no unambiguous detection of an FFLO state in a superconductor, though its signatures have been detected ultra-cold ${ }^{6} \mathrm{Li}$ gas [172]. This is likely because the conditions under which it can be formed are rather stringent. The superconductor must be strongly type-II and have a small Maki parameter. Additionally it must be very clean, because the FFLO state is readily destroyed by disorder [170]. The 
strongest contenders for fulfilling these criteria are heavy fermion superconductors and organic superconductors. The status of the search for such a phase in these materials is reviewed in detail by Matsuda and Shimahara in reference [170]. Although there is a great deal of indirect evidence suggesting the existence of an FFLO phase, there is no consensus that an FFLO phase has been observed.

The states considered by FFLO are just two of a number of possible PDW states. By writing down the Ginzburg-Landau expansion for the free-energy density of the PDW we can examine the range of possible states. For a quasi-two dimensional system with square lattice planes this is

$$
\begin{aligned}
& f=\alpha \sum_{i}\left|\Psi_{\vec{Q}_{i}}\right|^{2}+\beta_{1}\left(\sum_{i}\left|\Psi_{\vec{Q}_{i}}\right|^{2}\right)^{2}+\beta_{2} \sum_{i<j}\left|\Psi_{\vec{Q}_{i}}\right|^{2}\left|\Psi_{\vec{Q}_{j}}\right|^{2} \\
& +\beta_{3}\left(\left|\Psi_{\vec{Q}_{x}}\right|^{2}\left|\Psi_{-\vec{Q}_{x}}\right|^{2}+\left|\Psi_{\vec{Q}_{y}}\right|^{2}\left|\Psi_{-\vec{Q}_{y}}\right|^{2}\right)+\beta_{4}\left[\Psi_{\vec{Q}_{x}} \Psi_{-\vec{Q}_{x}}\left(\Psi_{\vec{Q}_{y}} \Psi_{-\vec{Q}_{y}}\right)^{*}+\left(\Psi_{\vec{Q}_{x}} \Psi_{-\vec{Q}_{x}}\right)^{*} \Psi_{\vec{Q}_{y}} \Psi_{-\vec{Q}_{y}}\right] \\
& +\kappa_{1} \sum_{i}\left|\vec{D} \Psi_{\vec{Q}_{i}}\right|^{2}+\kappa_{2} \sum_{i}(-1)^{i}\left[\left|D_{x} \Psi_{\vec{Q}_{i}}\right|^{2}-\left|D_{y} \Psi_{\vec{Q}_{i}}\right|^{2}\right]+\kappa_{3} \sum_{i}\left|D_{z} \Psi_{\vec{Q}_{i}}\right|^{2}+\frac{1}{2}(\nabla \times \vec{A})^{2},
\end{aligned}
$$

where $(-1)^{i}$ is -1 for $\vec{Q}_{i}= \pm \vec{Q}_{x}$ and 1 for $\vec{Q}_{i}= \pm \vec{Q}_{y}$ with $\vec{D}=-i \nabla-2 e \vec{A}$ and $\vec{B}=\nabla \times \vec{A}$.

The possible PDW phases resulting from this free-energy density in equation 6.6 are listed in table 6.1. The FF and LO states discussed above are numbered 1 and 3 respectively. In section 6.4 I will explicitly discuss the coupling of the PDW order parameter to that of a charge density wave (CDW) and spin density wave (SDW), with the result that different PDW states will induce CDW and SDW at different wave-vectors. These results are summarised in table 6.1 and may be used as a tool to differentiate between different PDW states [167].

Clearly the case considered by FFLO is a very special situation and in general we do not expect PDW formation in weakly coupled superconductors. However, we might still expect a divergent susceptibility to a PDW state to arise from a strongly correlated many-body state. It has been proposed that a PDW exists in the cuprates for this very reason. In the following sections I will review the experimental evidence and theoretical proposals for the cuprate PDW. 


\begin{tabular}{|c|c|c|c|c|c|c|}
\hline Phase & $\Psi_{\vec{Q}_{x}}$ & $\Psi_{\vec{Q}_{y}}$ & $\Psi_{-\vec{Q}_{x}}$ & $\Psi_{-\vec{Q}_{y}}$ & CDW Modes & SDW Modes \\
\hline 1 & $e^{i \phi_{1}}$ & 0 & 0 & 0 & None & None \\
\hline 2 & $e^{i \phi_{1}}$ & $e^{i \phi_{2}}$ & 0 & 0 & $\rho_{\vec{Q}_{x}-\vec{Q}_{y}}$ & $S_{\vec{Q}_{x}-\vec{Q}_{y}}^{Z}$ \\
\hline 3 & $e^{i \phi_{1}}$ & 0 & $e^{i \phi_{2}}$ & 0 & $\rho_{2 \vec{Q}_{x}}$ & None \\
\hline 4 & $e^{i \phi_{1}}$ & $e^{i \phi_{2}}$ & $e^{i \phi_{3}}$ & $e^{i\left[\phi_{1}+\phi_{3}-\phi_{2}\right]}$ & $\rho_{2 \vec{Q}_{x}}, \rho_{2 \vec{Q}_{y}}, \rho_{\vec{Q}_{x}-\vec{Q}_{y}}$ & None \\
\hline 5 & $e^{i \phi_{1}}$ & $i e^{i \phi_{2}}$ & $e^{i \phi_{3}}$ & $i e^{i\left[\phi_{1}+\phi_{3}-\phi_{2}\right]}$ & $\rho_{2 \vec{Q}_{x}}, \rho_{2 \vec{Q}_{y}}$ & $S_{\vec{Q}_{x}-\vec{Q}_{y}}^{Z}, S_{\vec{Q}_{x}+\vec{Q}_{y}}^{Z}$ \\
\hline
\end{tabular}

TABLE 6.1: Possible PDW phases (numbered 1-5) allowed by the free energy density in equation 6.6. These phases will induce CDW and SDW at the wave-vectors listed in the table due to their symmetry allowed coupling to the PDW in the free energy density as detailed in section 6.4.

\subsection{Experimental Evidence for a Cuprate PDW}

Prior to the new results presented in chapter 7 there was no direct evidence for a spatially modulating pairing amplitude in the cuprates. However, there were many intriguing pieces of indirect experimental evidence that led to proposals of a PDW in cuprates. In this section I will review this evidence before going on to discuss the various theoretical proposals for the cuprate PDW state in the next section.

\subsubsection{Decoupling of Superconductivity between the $\mathrm{CuO}_{2}$ Planes}

The strongest evidence for a PDW state comes from the group of cuprate materials based on the insulator $\mathrm{La}_{2} \mathrm{CuO}_{4}$. Many of these materials form "stripe" order where co-linear anti-ferromagnetic spin and charge modulations $\vec{Q}_{s}$ and $\vec{Q}_{c}$ exist with the relationship $\overrightarrow{Q_{c}}=2 \vec{Q}_{s} \approx\left(\frac{1}{4}, 0\right) ;\left(0, \frac{1}{4}\right)[40]$. The stripe order is strongest in the vicinity of hole-doping $p \approx 1 / 8$ where there is an anomalous suppression in the bulk superconducting $T_{c}$, known as the "1/8th anomaly" [41]. The principal observation pointing to PDW order in these materials is that there are many signatures that the individual $\mathrm{CuO}_{2}$ planes become superconducting at a temperature much higher than the bulk $T_{c}$.

In $\mathrm{La}_{2-x} \mathrm{Ba}_{x} \mathrm{CuO}_{4}$ a large anisotropy between the in-plane resistivity, $\rho_{a b}$, and outof-plane resistivity, $\rho_{c}$, onsets at the same temperature as spin-stripe order $T_{\mathrm{spin}}=$ $42 \mathrm{~K}$ [173]. This anisotropy is most pronounced in the temperature range $10 \mathrm{~K}<T<$ 16K. Here $\rho_{a b}$ is, to within error, zero; whilst $\rho_{c}$ has a value characteristic of a bad metal $(1-10 \mathrm{~m} \Omega \mathrm{cm})$. For $16 \mathrm{~K}<T<T_{\text {spin }}, \rho_{a b}$ exhibits a temperature dependence that 
fits that predicted above a Berezinskii-Kosterlitz-Thouless transition, indicating that superconducting fluctuations are confined to the $2 \mathrm{D}$ planes.

Similarly, below $T_{\text {spin }}$ a diamagnetic response is seen in the magnetic susceptibility for fields perpendicular to the $\mathrm{CuO}_{2}$ planes (requiring screening currents in the plane) whereas none is seen for a field within the planes (requiring screening currents along the c-axis) [174]. Together, these observations are highly indicative of the fact that superconductivity onsets within the $\mathrm{CuO}_{2}$ planes at a temperature significantly higher than the bulk $T_{c}$.

On tuning, by chemical doping, to within the stripe-ordered region of the phase diagram there is also strong suppression of the superfluid stiffness along the c-axis [175]. The layered, quasi-2D nature of the cuprate superconductors means that their response to an electric field polarised along the c-axis can be modeled as a stack of intrinsic Josephson junctions where the $\mathrm{CuO}_{2}$ planes are the superconducting electrodes, separated by weak links formed by the dopant layers [176].

As discussed in section 5.3.1, the dynamics of a Josephson junction are analogous to that of a damped pendulum with resonant Josephson plasma frequency $\hbar \omega_{J}=\sqrt{2 E_{J} E_{C}}$, where $E_{J}$ is the Josephson coupling energy and $E_{C}$ the capacitive charging energy. In c-axis polarised optical spectroscopy, this leads to an adsorption edge with frequency proportional to $\sqrt{E_{J}}$ for fixed interlayer capacitance. This is known as the Josephson plasma resonance.

In $\mathrm{La}_{1.85-y} \mathrm{Nd}_{y} \mathrm{Sr}_{0.15} \mathrm{CuO}_{4}$ the Josephson plasma resonance rapidly shifts to lower frequencies as y is increased towards the stripe-ordered region. Upon exceeding the critical $\mathrm{Nd}$ concentration where stripe-order is stabilised, the Josephson plasma resonance all but disappears [175]. Furthermore, when a c-axis magnetic field is applied to $\mathrm{La}_{1.9} \mathrm{Sr}_{0.1} \mathrm{CuO}_{4}$ there is a pronounced reduction of the Josephson plasma resonance frequency while the in-plane superfluid density shows little change [177]. These results point towards a situation where entry into the stripe-ordered phase strongly suppresses the Josephson coupling between adjacent $\mathrm{CuO}_{2}$ planes, so that the bulk $T_{c}$ for $3 \mathrm{D}$ superconductivity is much less than that at which the $\mathrm{CuO}_{2}$ planes themselves become superconducting.

It has been proposed that the frustration of interlayer Josephson coupling needed to explain these anomalous observations would naturally be provided by the existence of 
a particular PDW state within the stripe-ordered region of the $\mathrm{La}_{2} \mathrm{CuO}_{4}$ family phase diagram $[178,179]$. What is known experimentally is that co-linear CDW and SDW order with wave-vectors along the $\mathrm{Cu}-\mathrm{O}$ directions exists with periodicity $\approx 4 \mathrm{a}_{0}$ and $8 \mathrm{a}_{0}$ respectively [40]. This is attributed to the segregation of holes in the $\mathrm{CuO}_{2}$ plane into regions of un-doped antiferromagnetic insulator separated by domain walls of holes with a periodicity of $4 \mathrm{a}_{0}[42]$.

Across the domain walls the $(\pi, \pi)$ anti-ferromagnetism undergoes a $\pi$ phase-shift resulting in an SDW periodicity of $8 a_{0}$. The picture put forward by Berg et al. is that the hole rich regions superconduct but are separated by regions of correlated insulator that result in a negative Josephson coupling between them. This leads to a $\pi$ phase-shift of the pairing amplitude across the insulating region. The result of this is a spatially modulated superconducting order parameter with periodicity $8 \mathrm{a}_{0}$ and a mean of zero.

In the low temperature tetragonal (LTT) phase of $\mathrm{La}_{2-x} \mathrm{Ba}_{x} \mathrm{CuO}_{4}$ where stripe-order is found there are two $\mathrm{CuO}_{2}$ planes per unit cell [180, 181]. It is widely thought that the stripes are orthogonal in adjacent planes and that the stripes are offset from each other by $2 \mathrm{a}_{0}$ in adjacent unit cells to minimise coulomb repulsion as shown in figure 6.3 [42]. This would lead to an exact cancellation of the Josephson coupling between the first, second and third neighbour planes [179].

Within the planes however, a sufficiently long range ordered PDW state would exhibit many of the same properties as a uniform superconductor such as zero resistance, a Meissner effect and flux quantisation [63], due to its broken gauge symmetry. As such, this specific PDW proposal for the stripe ordered cuprates would offer an explanation for the observed resistivity anisotropy and suppression of the Josephson plasma resonance.

Is is natural to ask whether cuprate materials beyond the $\mathrm{La}_{2} \mathrm{CuO}_{4}$ family exhibit the same phenomenology, thus suggesting that a PDW is ubiquitous in cuprates. The answer is that there are similar signatures but they are much less clear. In $\mathrm{YBa}_{2} \mathrm{Cu}_{3} \mathrm{O}_{6+\delta}$ there is evidence for a Josephson plasma resonance at temperatures far above the bulk $T_{c}$ [182]. There are also magnetisation studies in high magnetic fields that are interpreted as pointing towards PDW correlations [183]. However, beyond the $\mathrm{La}_{2} \mathrm{CuO}_{4}$ family of cuprates, the most persuasive arguments for the existence of a PDW come from the interpretation of photoemission spectra, which I will now discuss. 

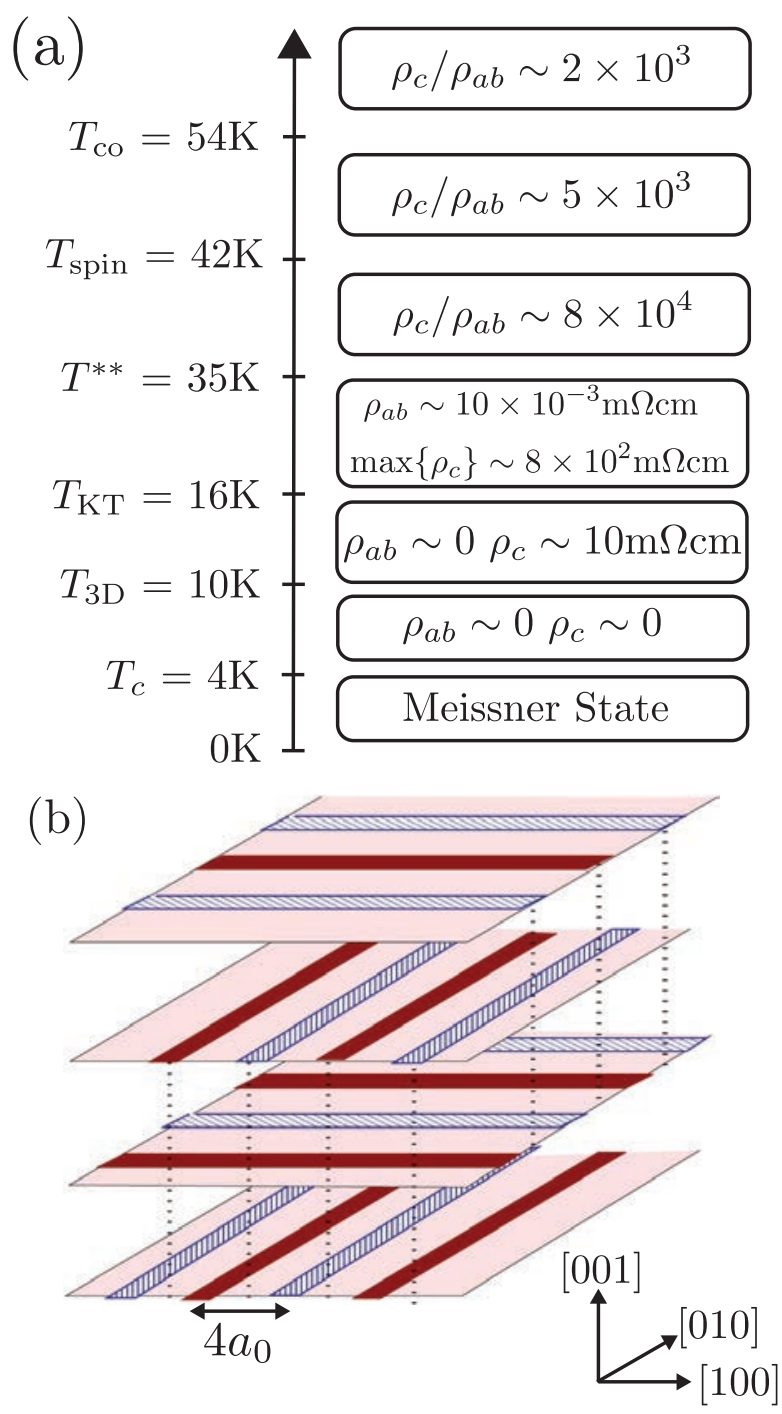

FiguRE 6.3: (a) Hierarchy of temperature scales associated with spin-stripe order at $x=1 / 8$ in $\mathrm{La}_{2-x} \mathrm{Ba}_{x} \mathrm{CuO}_{4}$. A large resistivity anisotropy sets in below $T_{\text {spin }}$. For $10 \mathrm{~K}<T<16 \mathrm{~K}$ the in plane resistivity, $\rho_{a b}$, is zero to within error whilst the out-ofplane resistivity, $\rho_{c}$, takes the value of a bad metal. This indicates that $\mathrm{CuO}_{2}$ planes themselves are superconducting at temperatures above $T_{c}$. A PDW state has been proposed to explain these observations [179]. (b) Schematic of the PDW state proposed

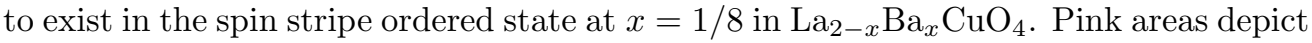
regions of un-doped antiferromagnetic insulator. Red and grey regions are domain walls between the insulator regions where holes aggregate at intervals of $4 a_{0}$ forming a CDW with $\vec{Q}_{C}=\left(\frac{1}{4}, 0\right) ;\left(0, \frac{1}{4}\right) \frac{2 \pi}{a_{0}}$. These hole-rich regions are superconducting. Red and grey depict a superconducting order parameter of opposite sign. The state is hence a PDW (of the LO type) with wave-vector $\vec{Q}_{P}=\left(\frac{1}{8}, 0\right) ;\left(0, \frac{1}{8}\right) \frac{2 \pi}{a_{0}}$. The stripes are thought to be orthogonal in nearest neighbour layers due to the LTT crystal structure $[40,180,181]$ and stripes in 2 nd nearest neighbour layers are offset by $2 \mathrm{a}_{0}$ [42]. The dashed lines demonstrate that this configuration leads to a cancellation of the c-axis Josephson coupling between first, second and third nearest neighbours. Figure adapted with permission from reference [179]. 


\subsubsection{Photoemission Spectra}

P. A. Lee has put forward a detailed argument that the anti-nodal gap present for $T<T^{*}$, as observed by ARPES on $\mathrm{Bi}_{2} \mathrm{Sr}_{2} \mathrm{CuO}_{6+\delta}$, is compatible with a PDW and incompatible with a CDW or uniform $d$-wave pairing [184].

At temperatures above $T^{*}$ a full Fermi surface is observed [185]. For a series of cuts parallel to $\vec{k}_{x}$ in momentum space (figure 6.4 (a)) the dispersion of this band is shown as red points in figures $6.4(\mathrm{~b})-(\mathrm{e})$. Clear Fermi momenta $k_{F 1}$ and $k_{F 2}$ can be determined from where this band crosses the Fermi energy.

For $T_{c}<T<T^{*}$ a pseudo-gap opens in the $(1,0)$ directions leaving only arcs of spectral weight along the sections of Fermi surface proximal to the $(1,1)$ directions [24]. The tips of these Fermi arcs are formed when a band moves up to touch the Fermi level from below. At the tip of the arc, the gap to excitations is above the chemical potential. This is incompatible with a mean-field CDW order parameter which would produce a gap below the Fermi energy at these locations $[184,186]$.

For $T<T_{c}$ the whole Fermi surface is gapped except at the nodes along the $(1,1)$ directions. Near these nodes a particle-hole symmetric gap is observed that is consistent with a $\mathrm{d}_{x^{2}-y 2}$ superconducting gap [185, 187-190]. The spectrum of excitations below the Fermi energy exhibits maxima at momenta $k_{G 1}$ and $k_{G 2}$ that are approximately equal to $k_{F 1}$ and $k_{F 2}$ [185]. Such "back-bending" is familiar from the Bogoliubov spectrum of the BCS Hamiltonian, discussed in section 1.1.2. However, in the anti-nodal region $k_{G 1}$ and $k_{G 2}$ diverge from $k_{F 1}$ and $k_{F 2}$ with increasing proximity to the anti-node [185]. This suggests that the anti-nodal gap does not have its origins in $\vec{Q}=0$ (uniform) superconductivity.

Taken together, these features indicate that the anti-nodal gap does not originate from a CDW or $\vec{Q}=0$ pairing. Lee explicitly calculates that for an $\approx 8 \mathrm{a}_{0}$ unit cell LO type PDW, driven by a mechanism he calls Amperean pairing (see section 6.5.3), all qualitative features of the photoemission spectra can be reproduced [184].

Having reviewed the experimental evidence for a PDW in cuprates, I will now discuss theoretical proposals for how it may arise. 

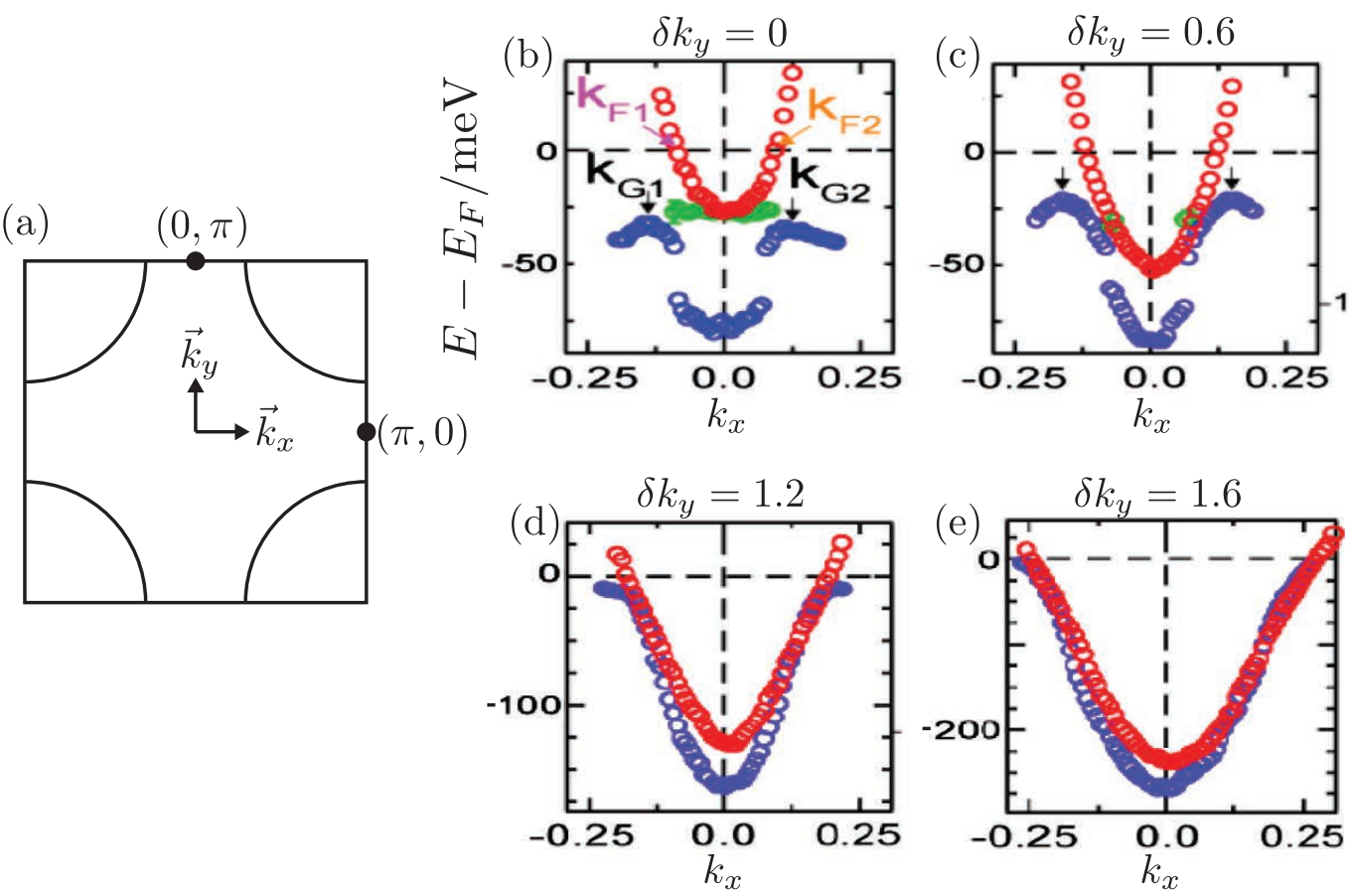

Figure 6.4: (a) Schematic Brillouin zone for the single layer cuprate $\mathrm{Bi}_{2} \mathrm{Sr}_{2} \mathrm{CuO}_{6+\delta}$ showing a full, hole-like, Fermi surface. (b)-(e) ARPES data on $\mathrm{Bi}_{2} \mathrm{Sr}_{2} \mathrm{CuO}_{6+\delta}$ from R. He et al. [185] reproduced with permission from reference [184]. The four panels show data extracted dispersions for cuts in momentum space parallel to $\vec{k}_{x}$ at $\delta \vec{k}_{y}=\pi-\vec{k}_{y}$. Red circles show the dispersion of the full Fermi surface observed for $T>T^{*}$. Fermi momenta $k_{F 1}$ and $k_{F 2}$ are extracted from the points where this band crosses the Fermi energy $\left(E_{F}\right)$. Blue circles show the dispersion of the band below the Fermi energy that is observed for $T<T_{c}$. It exhibits maxima at momenta $k_{G 1}$ and $k_{G 2}$ that diverge from $k_{F 1}$ and $k_{F 2}$ with increasing proximity to the anti-node. This indicates that the anti-nodal gap is not a result of uniform $d$-wave pairing (see text).

\subsection{Coupled Order Parameters}

The cuprates habour many putative broken symmetries. The list includes superconducting phase, lattice translational, point group and time-reversal symmetries as well as combinations thereof [22]. Ideally we would like to formulate a description of (at least parts of) the cuprate phase diagram in terms of a minimal set of order parameters whose couplings to each other produce the full complexity of the system. In this section I will discuss how PDW order couples to other order parameters and hence how it may fit into such a framework.

A common situation in modern condensed matter physics is to be faced with a material where multiple degrees of freedom break a symmetry and to ask the question "which degrees of freedom are in the driving seat and which go along for the ride?" $[168,191]$. 
It is sometimes useful to cast this question in terms of primary and secondary order parameters. To understand what that means one can consider the following free energy expansion,

$$
F\left[\Psi_{1}, \Psi_{2}\right]=\frac{1}{2} \chi_{1}^{-1} \Psi_{1}^{2}+\frac{1}{4} b \Psi_{1}^{4}+\frac{1}{2} \chi_{2}^{-1} \Psi_{2}^{2}+\lambda_{12} \Psi_{1} \Psi_{2}+\ldots,
$$

where $\Psi_{1}$ and $\Psi_{2}$ are real order parameters. $\chi_{1}^{-1}$ goes to zero at $T=T_{c}$ and is negative for $T<T_{c}$. In contrast, $\chi_{2}^{-1}$ and $b$ are positive for all $T$. For $T<T_{c}$ the free energy will be minimised by $\Psi_{1}$ taking on a non-zero value and spontaneously breaking a $\mathbb{Z}_{2}$ symmetry. $\mathbb{Z}_{n}$ refers to an invariance of the free energy under rotations by $2 \pi / n$, which for $n=2$ is the symmetry of the Ising model.

Considering the third term in equation 6.7 in isolation, it always raises the free energy to make $\Psi_{2} \neq 0$. However, if you consider the coupling term $\lambda_{12} \Psi_{1} \Psi_{2}$ then as long as $\Psi_{1} \neq 0$ there is always some small non-zero $\Psi_{2}$,

$$
\Psi_{2}=-\chi_{2} \lambda_{12} \Psi_{1}
$$

that lowers the free energy regardless of the sign of $\lambda_{12}$. This is because $\Psi_{2}$ enters the Landau expansion at linear order. Thus in this example, $\Psi_{2}$ orders concurrently with $\Psi_{1}$ even though it would never have ordered in and of itself. Here we would term $\Psi_{1}$ a primary order parameter that induces secondary order in $\Psi_{2}$.

The question of which order parameter is in the driving seat is only meaningful if the coupling $\lambda_{12}$ is relatively small. Otherwise the reduction in free energy from the coupling term can be comparable to that of the quadratic term of the "primary" order parameter. In this case neither order parameter is "in the driving seat", rather they are "intertwined" in a single microscopic instability towards both orders [168].

Nevertheless it can still be useful to describe the thermodynamics of a system in terms of a set of primary order parameters whose products form accompanying secondary orders. For example, as I will shown in section 6.4 , a CDW with wave-vector $2 \vec{Q}$ can be expressed as a product of PDW order parameters $\Psi_{-\vec{Q}}^{*} \Psi_{\vec{Q}}$. While this is largely a matter of convenience and economy, if a PDW exists anywhere in the phase diagram (in a region where $\Psi_{0}=0$ ) then it should be regarded as primary. A pure PDW phase can only be 
expressed as a composite of uniform superconductivity and CDW by fractionalisation [168].

This prescription also allows for the fact that these secondary order parameters, which are expressed as products of the primary order parameters, may have non-zero expectation even if the expectation of the primary order parameters themselves vanish. This can occur because for a general observable $O,\left\langle O^{2}\right\rangle \not \equiv\langle O\rangle^{2}$.

For instance, a stripe-CDW $\left(\left\langle\Psi_{\vec{Q}_{x}}\right\rangle \neq 0,\left\langle\Psi_{\vec{Q}_{y}}\right\rangle=0\right)$ breaks rotational and translational symmetry. This stripe-CDW phase can "melt" via two transitions [192]. At the first transition, translation symmetry is restored $\left(\left\langle\rho_{\vec{Q}_{x}}\right\rangle=0\right)$ but rotational symmetry is still broken in what is called a nematic phase. In the nematic phase the rotational symmetry breaking is encoded in the nematic order parameter $\mathcal{N}=\left\langle\left|\rho_{x}\right|^{2}\right\rangle-\left\langle\left|\rho_{y}\right|^{2}\right\rangle$ which is nonzero even though $\left\langle\rho_{x}\right\rangle=\left\langle\rho_{y}\right\rangle=0$ [192]. At higher temperatures still a second transition will restore the rotational symmetry.

Having considered conceptually how order parameters couple, I will now go on to discuss the coupling of the PDW to other order parameters.

\subsection{Coupling of PDW to Other Order Parameters}

In discussing the coupling of a PDW to other order parameters I will, for brevity, consider only uniform superconductivity, CDW and SDW. These are ordering tendencies that are unambiguously experimentally confirmed to exist at zero magnetic field in at least some, if not all, cuprate materials $[58,111]$.

\subsubsection{Coupling to CDW}

CDW and PDW order parameters $\rho_{\vec{Q}}$ and $\Psi_{-\vec{Q}}$ may not couple bi-linearly. Although they carry opposite momenta, making the product $\rho_{\vec{Q}} \Psi_{-\vec{Q}}$ translationally invariant, this term is not invariant under phase symmetry transformations. The lowest order coupling that can be admitted is a product of two PDW and one CDW order parameters [167]. The CDW therefore provides an additional contribution to the free energy

$$
F_{\mathrm{CDW}}=\sum_{i, j}\left\{\frac{1}{2} \rho_{i, j} \chi_{C, \vec{Q}_{i}}^{-1} \rho_{\vec{Q}_{i}} \rho_{-\vec{Q}_{j}}+\lambda_{i, j}^{C} \rho_{\vec{Q}_{j}-\vec{Q}_{i}}\left[\Psi_{\vec{Q}_{i}} \Psi_{\vec{Q}_{j}}^{*}+\Psi_{-\vec{Q}_{j}} \Psi_{-\vec{Q}_{i}}^{*}\right]\right\}+\ldots
$$


which allows for a PDW to induce a CDW of the form

$$
\rho_{\vec{Q}_{i}-\vec{Q}_{i}}=-\chi_{C, \vec{Q}_{i}-\vec{Q}_{j}} \lambda_{i, j}^{C}\left[\Psi_{\vec{Q}_{i}} \Psi_{\vec{Q}_{j}}^{*}+\Psi_{-\vec{Q}_{j}} \Psi_{-\vec{Q}_{i}}^{*}\right]
$$

This implies that, for example, a uni-directional PDW $\left\{\Psi_{\vec{Q}_{x}}, \Psi_{\vec{Q}_{y}}, \Psi_{-\vec{Q}_{x}}, \Psi_{-\vec{Q}_{y}}\right\}=$ $\left\{e^{i \phi_{1}}, 0, e^{i \phi_{2}}, 0\right\}$ will induce a uni-directional CDW with wave-vector $2 \vec{Q}$. The converse is not necessarily true because the PDW appears at quadratic order in this term.

\subsubsection{Coupling to SDW}

A PDW may only induce SDW order if it itself breaks time-reversal symmetry. Furthermore the types of PDW order described by eqn. 6.6 may only couple to the $z$-component of spin if the coupling is to be invariant under time-reversal [167]. The additional contribution to the free energy takes the form

$$
F_{\mathrm{SDW}}=\sum_{i, j}\left\{\frac{1}{2} \delta_{i, j} \chi_{S, \vec{Q}_{i}}^{-1} S_{\vec{Q}_{i}}^{z} S_{-\vec{Q}_{j}}^{z}+\lambda_{i, j}^{S} S_{\vec{Q}_{j}-\vec{Q}_{i}}^{z} i\left[\Psi_{\vec{Q}_{i}} \Psi_{\vec{Q}_{j}}^{*}-\Psi_{-\vec{Q}_{j}} \Psi_{-\vec{Q}_{i}}^{*}\right]\right\}
$$

which allows for a PDW to induce an SDW of the form

$$
S_{\vec{Q}_{i}-\vec{Q}_{i}}^{z}=-\chi_{S, \vec{Q}_{i}-\vec{Q}_{j}} \lambda_{i, j}^{S}\left[\Psi_{\vec{Q}_{i}} \Psi_{\vec{Q}_{j}}^{*}-\Psi_{-\vec{Q}_{j}} \Psi_{-\vec{Q}_{i}}^{*}\right]
$$

In contrast to the coupling to $\mathrm{CDW}$, the uni-directional PDW with $\left\{\Psi_{\vec{Q}_{x}}, \Psi_{\vec{Q}_{y}}, \Psi_{-\vec{Q}_{x}}, \Psi_{-\vec{Q}_{y}}\right\}=\left\{e^{i \phi_{1}}, 0, e^{i \phi_{2}}, 0\right\}$ does not induce any SDW order because it does not itself break time-reversal symmetry. However, a bi-directional PDW $\left\{\Psi_{\vec{Q}_{x}}, \Psi_{\vec{Q}_{y}}, \Psi_{-\vec{Q}_{x}}, \Psi_{-\vec{Q}_{y}}\right\}=\left\{e^{i \phi_{1}}, e^{i \phi_{2}}, 0,0\right\}$ would induce SDW with wave-vector $\vec{Q}_{x}-\vec{Q}_{y}$. The induced CDW and SDW for the stable PDW phases of the free energy in eqn. 6.6 are summarised in table 6.1 .

\subsubsection{Coupling to Uniform Superconductivity}

Finally I will now consider the coupling of a PDW to a uniform charge-2e $d$-wave superconducting order parameter $\Psi_{0}$. The lowest order direct coupling between the two is bi-quadratic because it is not possible to construct a bi-linear product of these order parameters that is simultaneously invariant under phase and translational symmetry 
transformations. However, if CDW and PDW with the same wave-vector, $\vec{Q}$, are present in the system then there is an allowed term that couples all three [63]:

$$
F_{\Psi}=\sum_{i, j} \delta_{i, j} \lambda_{i, j}^{\Psi}\left[\Psi_{0}^{*} \rho_{\vec{Q}_{i}} \Psi_{-\vec{Q}_{j}}+\Psi_{0} \rho_{\vec{Q}_{i}}^{*} \Psi_{-\vec{Q}_{j}}^{*}\right]
$$

A corollary of eqn. 6.13 is that if any two of the order parameters $\Psi_{0}, \Psi_{-\vec{Q}}$ and $\rho_{\vec{Q}}$ are non-zero, they will induce ordering in the third. For example, if a material has an instability towards co-existing superconducting and CDW orders then there must be some PDW induced that reflects the influence of the new periodicity introduced by the CDW on superconductivity. This result will be very important for the conclusions drawn at the end of this chapter.

This section has shown that many secondary order parameters can be induced as products of primary PDW order parameters. In the next section I will discuss various theoretical proposals for PDW order in cuprates and its proposed relationship to other broken symmetries in the phase diagram.

\subsection{Theoretical Proposals for a Cuprate PDW}

Physical reasoning for the existence of translational symmetry breaking in cuprates typically falls into one of three categories and the same is true of the PDW. In the first, hole doping leads to frustration of a Mott insulator and mesoscopic phase separation results [193]. In the second these phases condense from a spin-liquid formed from doping the Mott insulator [194]. In a third picture, the problem is approached from the opposite end of the doping axis. In these approaches the multitude of phases in the under-doped region are seen as instabilities of the itinerant metal with a full Fermi surface that exists in the over-doped region of the phase diagram [103, 195].

Theories for PDW falling into these categories will be discussed below. First however, I will examine the evidence from numerics for the existence of a PDW. 


\subsubsection{Variational Numerical Calculations}

The lowest energy variational solution to date for the $t-J$ model on the square lattice at intermediate coupling is that of Corboz et al. [196]. For $J / t$ between 0.2 and 0.8 and hole doping $p<0.16$ they found two modulated superconducting states that, within the accuracy of the method used, could feasibly be the ground state. Two of these states are shown in figure 6.5.

The first of these (figure 6.5 (a)) consists of a uniform $d$-wave superconducting component $\left\langle\Psi_{0}\right\rangle \neq 0$ coexisting with $\mathrm{CDW}\left\langle\rho_{2 \vec{Q}}\right\rangle \neq 0$. This state necessarily also has a PDW component $\left\langle\Psi_{2 \vec{Q}}\right\rangle \neq 0$ (see equation 6.13) that, in this case, is in-phase with the CDW. The other state (figure $6.5(\mathrm{~b})$ ) is a pure-PDW where $\left\langle\Psi_{0}\right\rangle=0$ and $\left\langle\Psi_{\vec{Q}}\right\rangle \neq 0$. Through equation 6.9 this necessarily has a CDW component $\left\langle\rho_{2 \vec{Q}} \neq 0\right\rangle$. Which, again is in-phase with the CDW. Both of these have co-existing SDW modulation of the antiferromagnetic spin texture. In both cases the nodes of the SDW lie at the maxima of the pairing amplitude.

(a)

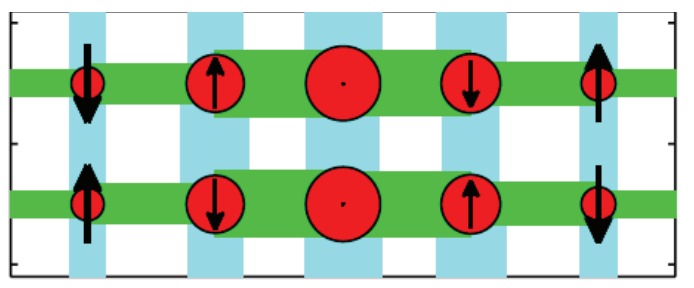

(b)

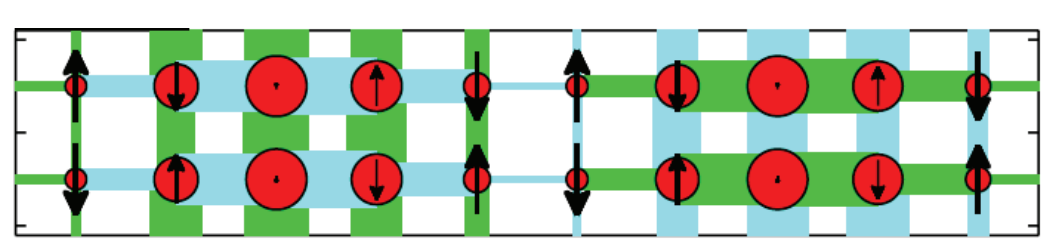

FiguRe 6.5: Striped variational solutions to the $t$ - $J$ model at intermediate coupling calculated using the iPEPS method by Corboz et al. [196]. The size of the red circles indicates the density of holes on that site. The thickness of the rectangles on the bonds indicate the magnitude of the pairing amplitude with green and blue indicating different signs of the amplitude. The length and orientation of the black arrows denotes the magnetic moment on each site. In (a), a state with $\left\langle\Psi_{0}\right\rangle \neq 0$ coexisting with CDW and $\left\langle\Psi_{2 \vec{Q}}\right\rangle$ and $\left\langle\rho_{2 \vec{Q}}\right\rangle \neq 0$ is depicted. In this case $2 \pi /|2 \vec{Q}|=5 a_{0}$. In (b) a pure PDW phase with $\left\langle\Psi_{0}\right\rangle=0,\left\langle\Psi_{\vec{Q}}\right\rangle \neq 0$ and $\left\langle\rho_{2 \vec{Q}}\right\rangle \neq 0$ is shown. The periodicity of the PDW is twice that in (a) as can be seen from the change in sign of the pairing amplitude every $5 a_{0}$. Reproduced from reference [196] with permission. 
In as much as the $t-J$ model at intermediate coupling accurately reflects the physics of the cuprates, this makes the existence of a spatially modulated pairing amplitude (with or without a uniform superconducting component) highly plausible.

\subsubsection{PDW within Mean-Field Models}

Several works have now found evidence for a PDW type ground state within mean-field type treatments of the superconducting state of strongly correlated electron systems [178, 197-202]. Striped phases where superconducting hole-rich regions are separated by regions of anti-ferromagnetism are found. These phases either have the pairingamplitude changing sign between adjacent regions $\left(\left\langle\Psi_{0}\right\rangle=0,\left\langle\Psi_{\vec{Q}}\right\rangle \neq 0,\left\langle\rho_{2 \vec{Q}}\right\rangle \neq 0\right)$ or maintaining the same sign $\left(\left\langle\Psi_{0}\right\rangle \neq 0,\left\langle\Psi_{\vec{Q}}\right\rangle=0,\left\langle\rho_{2 \vec{Q}}\right\rangle \neq 0,\left\langle\rho_{2 \vec{Q}}\right\rangle \neq 0\right)$.

\subsubsection{Amperean Pairing}

In the preceding section I have discussed attempts to establish a PDW as the ground state of a realistic model of the cuprates. Some authors have instead explored the consequences of a physically motivated ansatz for the form of PDW order that may occur. One such model would be the "Amperean Pairing" proposal of Patrick Lee which is based on a resonating valence bond (RVB) description of the cuprate pseudo-gap [184, 194, 203].

In some RVB type theories the electron is decomposed into a spinless charged boson (the holon) and a neutral spin- $1 / 2$ fermion (the spinon) which are coupled by a fluctuating gauge field [194]. It has been shown that a uniform $d$-wave superconducting state can arise from this kind of liquid [204]. In this picture the holons Bose condense and the spinons form a Fermi surface. The spinons may then form $d$-wave pairs via an attractive interaction mediated by the gauge bosons.

The spinons are not charged, meaning that this is not a true superconductor. However the Bose condensation of the charged holons converts the spinons to electrons with a reduced spectral weight and turns this state into a true superconductor [194].

The coupling of the spinons to the transverse gauge field is strong and unscreened. In electromagnetism a current of charged particles creates a transverse magnetic (gauge) 
field. If there is a second particle with parallel momentum, the particles will experience an attractive force, described by Ampere's law. Similarly, spinons of parallel momentum experience an attractive interaction through the transverse gauge fields they create [205]. P. Lee has argued that pairing of spinons with nearly parallel momenta, mediated by this interaction, can lead to the formation of a PDW state through a process called "Amperean pairing" [184]. As shown in figure 6.6 (a), pairing between spinons in the anti-nodal region of the Brillouin zone in cuprates can lead to Cooper-pairs with non-zero centre of mass momentum: a PDW.

In this picture, the wave-vector of the PDW would be determined by the separation between pieces of the Fermi surface in the anti-nodal region. It would hence consist of a state with PDW order parameter $\left\langle\Psi_{\vec{Q}}\right\rangle \neq 0$ where $\vec{Q}$ decreases with increasing hole-doping and is approximately equal to $\left(\frac{2 \pi}{8 a_{0}}, 0\right) ;\left(0, \frac{2 \pi}{8 a_{0}}\right)$. Through equation 6.9 this would necessarily imply an accompanying $\mathrm{CDW} \rho_{2 \vec{Q}}$ which would be be in agreement with experiment in $\mathrm{YBa}_{2} \mathrm{Cu}_{3} \mathrm{O}_{6+x}, \mathrm{Bi}_{2} \mathrm{Sr}_{2} \mathrm{CaCu}_{2} \mathrm{O}_{8}, \mathrm{Bi}_{2} \mathrm{Sr}_{2} \mathrm{CuO}_{6+\delta}, \mathrm{Na}_{x} \mathrm{Ca}_{2-x} \mathrm{CuO}_{2} \mathrm{Cl}_{2}$ and $\mathrm{HgBa}_{2} \mathrm{CuO}_{4+\delta}[58]$.

Within this picture charge order is an entirely subsidiary effect to PDW formation. A schematic phase diagram resulting from the Amperean pairing scenario is shown in figure 6.6 (b). The PDW, rendered short-range by disorder, onsets at $T_{\mathrm{PDW}-\mathrm{MF}}$, resulting in a pseudo-gap (blue region). At a lower temperature, $T_{\mathrm{CDW}}$, a secondary short-range CDW, $\rho_{2 \vec{Q}}$, onsets. On entering the uniform superconducting phase (dSC) the PDW and secondary CDW persist but with amplitude that decreases with temperature as a result of Fermi surface competition between PDW and d-SC. This is consistent with the gradual decreases in scattering intensity at $2 \vec{Q}$ observed by x-ray scattering in $\mathrm{YBa}_{2} \mathrm{Cu}_{3} \mathrm{O}_{6+x}$ [124]. As outlined in section 6.2.2, Lee argues argued that this Amperean PDW state would explain many of the peculiar features of the APRES data in $\mathrm{Bi}_{2} \mathrm{Sr}_{2} \mathrm{CuO}_{6+\delta}$, including a Fermi arc $[184,185]$.

Having considered this instability of an exotic spinon Fermi surface, I next discuss an instability to PDW order arising from a more conventional one. 
(a)

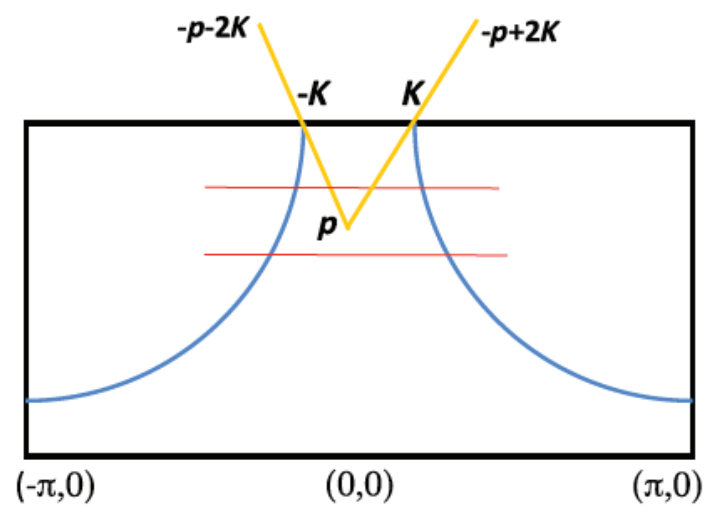

(b)

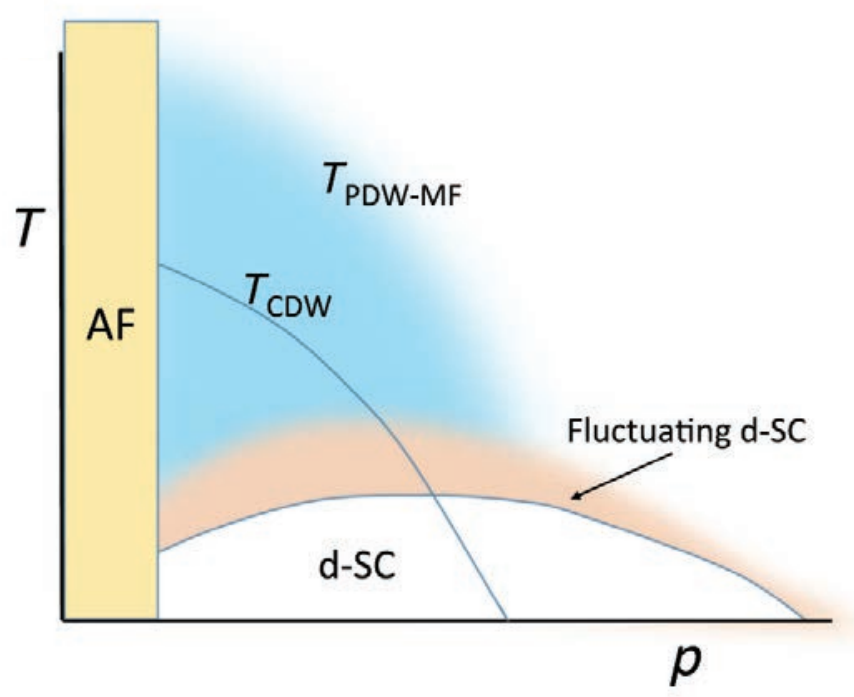

Figure 6.6: (a) Cartoon diagram of Amperean pairing reproduced from reference [184] with permission. Momenta are measured with respect to the $(0, \pi)$ point. Fermions near the point $\vec{K}$ with momenta $\vec{p}$ and $-\vec{p}+2 \vec{K}$ form a Cooper pair with centre of mass momentum $\vec{Q}=2 \vec{K}$. Similarly those near $-\vec{K}$ with momenta $\vec{p}$ and $-\vec{p}-2 \vec{K}$ form a Cooper pair with centre of mass momentum $\vec{Q}=-2 \vec{K}$. The non-zero centreof-mass momenta of the Cooper-pairs leads to a PDW. (b) Schematic phase diagram in Amperean pairing scenario reproduced from [184]. PDW, rendered short-range by disorder, onsets at $T_{\text {PDW-MF }}$ resulting in a pseudo-gap (blue region). At a lower temperature, $T_{\mathrm{CDW}}$, a secondary short-range CDW, $\rho_{2 \vec{Q}}$, onsets. On entering the uniform $d$-wave superconducting phase (d-SC) the PDW and secondary CDW persist but with amplitude that decreases with temperature as a result of Fermi surface competition between PDW and d-SC. 


\subsubsection{Spin-Fermion "Hot-Spot" Models}

Spin-Fermion models of the cuprates take a complete hole-like Fermi surface and couple the fermions via $(\pi, \pi)$ spin-fluctuations in the vicinity of an anti-ferromagnetic quantum critical point. These $(\pi, \pi)$ fluctuations strongly couple eight points on the Fermi surface where it intersects the anti-ferromagnetic zone boundary [117].

These interactions can give rise to $d$-wave superconducting, CDW and PDW orders. However, it is well known that these models do not have a leading instability at the mean-field level to CDW or PDW at the experimentally observed wave-vector, $(2 Q, 0) ;(0,2 Q)$, instead favouring those at $(2 Q, 2 Q)[97,103,117]$. Nevertheless, by studying sub-dominant instabilities towards order at $(2 Q, 0) ;(0,2 Q)$ it is hoped that insight into the nature of the superconducting and density wave states formed can be obtained [95, 99, 206, 207].

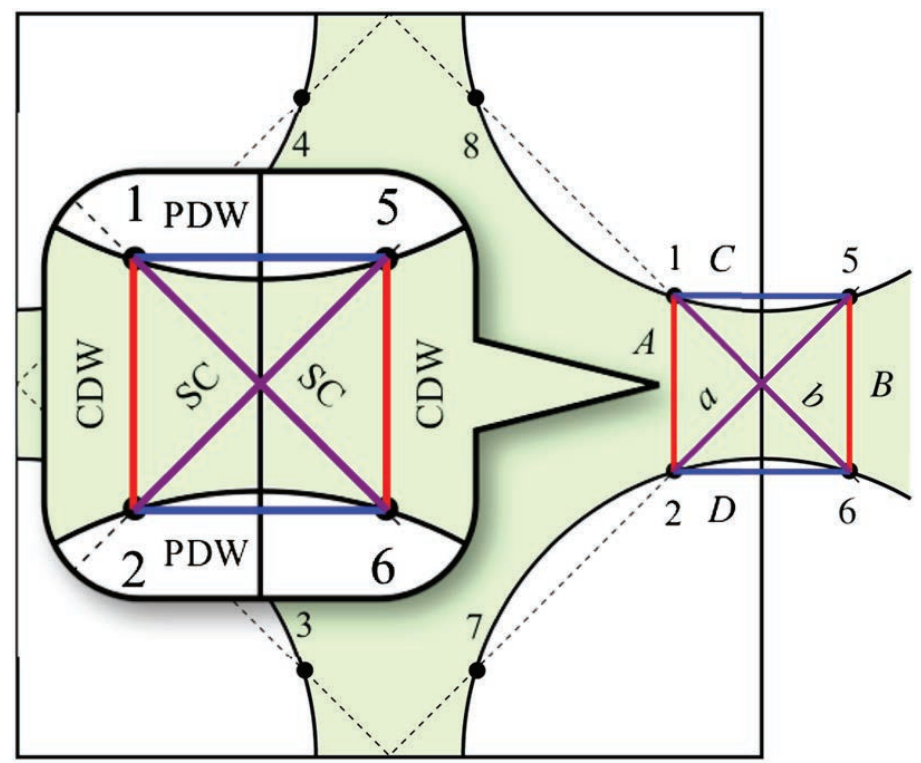

Figure 6.7: Cartoon of an orthogonal CDW/PDW state in a spin-fermion model reproduced from [207]. $(\pi, \pi)$ spin-fluctuations strongly couple eight "hot-spots" on the Fermi surface where it intersects the anti-ferromagnetic zone boundary. These interactions can give rise to $d$-wave superconducting, CDW and PDW orders. Here a PDW component with wave-vector $(2 Q, 0)$ is created by the pairing of hot fermions separated long the $\mathrm{x}$-direction (blue lines). A CDW component with wave-vector $(0,2 Q)$ is created by hot-spots separated along the y-direction (red lines). The CDW and PDW have the same wave-vector because a PDW field carries the total momentum of the paired fermions whereas a CDW field carries the momentum transfer. Within this type of model uniform $d$-wave superconductivity arises by pairing of hot fermions diagonally opposite from each other. 
It was noticed that if the non-linearity of the dispersion and Fermi surface curvature are neglected, spin-fermion models have an emergent SU(2) symmetry linking uniform $d$-wave superconductivity and $d$-symmetry form factor CDW with wave-vector $(2 Q, 2 Q)$ $[97,103,117]$. This means that these orders are exactly degenerate. In a similar vein, a symmetry between CDW and PDW at $(2 Q, 0) ;(0,2 Q)$ has been established [95, 207]. It has also been shown that for large values of the spin-fermion coupling, uni-directional (stripe) CDW/PDW order is preferred over bi-directional (chequerboard) order [95].

Wang et al. considered the scenario depicted in figure 6.7. In their model, a mixed CDW/PDW order develops where the CDW and PDW (of the LO type) are orthogonal to each other. With reference to figure 6.7 (a), hot fermions separated along the x-direction, say, will develop PDW order at $(2 Q, 0)$ whereas those along y would develop CDW order at $(0,2 Q)$. In doing so they spontaneously break the $C_{4}$ lattice rotation symmetry. Time-reversal symmetry is also broken due to spontaneous currents associated with the CDW [95].

The attraction of this model is that CDW and PDW appear at the same wave-vector (modulo $90^{\circ}$ rotation), which decreases with increasing hole doping. This is consistent with the wave-vectors observed by STM and scattering techniques in $\mathrm{YBa}_{2} \mathrm{Cu}_{3} \mathrm{O}_{6+x}$, $\mathrm{Bi}_{2} \mathrm{Sr}_{2} \mathrm{CaCu}_{2} \mathrm{O}_{8}, \mathrm{Bi}_{2} \mathrm{Sr}_{2} \mathrm{CuO}_{6+\delta}, \mathrm{Na}_{x} \mathrm{Ca}_{2-x} \mathrm{CuO}_{2} \mathrm{Cl}_{2}$ and $\mathrm{HgBa}_{2} \mathrm{CuO}_{4+\delta}$ [58]. Wang et al. also show that this orthogonal PDW/CDW state is consistent with the anti-nodal dispersion observed in the ARPES data of He et al. that motivated the Amperean pairing proposal (see section 6.2.2). Finally, because this model breaks $C_{4}$ rotational and time-reversal symmetry it is consistent with reports of such symmetry breaking in cuprates $[32-37]$.

\subsection{Experimentally Distinguishing PDW Scenaria}

In the previous section I discussed models which are, to varying degrees of approximation, representative of cuprate physics and show evidence for the existence of a PDW state. Where they differ is in the relationship of CDW to the PDW and whether the PDW exists with or without a uniform superconducting component.

Without reference to microscopics we can categorise these models into three groups as summarised in table 6.2. In all three scenaria uniform $d$-wave superconductivity is taken 


\begin{tabular}{|c|c|c|c|c|c|}
\hline Scenario & Temperature & $\begin{array}{c}\text { Primary } \\
\text { PDW }\end{array}$ & $\begin{array}{c}\text { Primary } \\
\text { CDW }\end{array}$ & $\begin{array}{c}\text { Secondary } \\
\text { PDW }\end{array}$ & $\begin{array}{c}\text { Secondary } \\
\text { CDW }\end{array}$ \\
\hline 1 & $T>T_{c}$ & $\vec{Q}$ & None & None & $2 \vec{Q}$ \\
\hline- & $T<T_{c}$ & $\vec{Q}$ & None & None & $2 \vec{Q}, \vec{Q}$ \\
\hline 2 & $T>T_{c}$ & $2 \vec{Q}$ & $2 \vec{Q}$ & None & None \\
\hline- & $T<T_{c}$ & $2 \vec{Q}$ & $2 \vec{Q}$ & None & None \\
\hline 3 & $T>T_{c}$ & None & $2 \vec{Q}$ & None & None \\
\hline- & $T<T_{c}$ & None & $2 \vec{Q}$ & $2 \vec{Q}$ & None \\
\hline
\end{tabular}

TABLE 6.2: Table characterising the possible PDW scenarios in cuprates by which order parameters are primary. Here $2 \vec{Q}=\vec{Q}_{C}$, the experimentally observed CDW wave-vector. PDW with wave-vector $\vec{Q}$ refers to $\Psi_{\vec{Q}}+\Psi_{-\vec{Q}}$ which is a uni-directional LO state (phase 3 in table 6.1). This is preferred in the models presented in section 6.5. PDWs of type 4 and 2 in table 6.1 would differ would differ from the type considered here by an additional CDW at $\vec{Q}_{x}-\vec{Q}_{y}$ which has never been experimentally detected. PDWs of type 5 and 2 in table 6.1 would differ from the type considered here by an additional SDW modulation at $\vec{Q}_{x}-\vec{Q}_{y}$ and also $\vec{Q}_{x}+\vec{Q}_{y}$ in the case of phase 2 . An FF type PDW (phase 1 in table 6.1) has no allowed CDW or SDW modes.

to be a primary order parameter. The three scenaria also include CDW order at the experimentally observed wave-vector $\vec{Q}_{C}=2 \vec{Q} \approx(0.25,0) \frac{2 \pi}{a_{0}}$ and $(0,0.25) \frac{2 \pi}{a_{0}}$, either as a primary order parameter or as secondary order expressed in terms of a primary PDW. Table 6.2 shows the relationship between the CDW and any PDW wave-vector present both above and below the uniform $T_{c}$.

As can be seen from table 6.2, by determining the relationship between the PDW wavevector and any CDW wave-vector(s) both above and below the uniform $T_{c}$ one can readily distinguish these scenarios. For instance, in scenario 1 , a new secondary CDW at wave-vector $\vec{Q}_{C} / 2$ is allowed upon cooling below $T_{C}$ which would positively identify this scenario.

Here I have outlined how to experimentally distinguish different PDW scenaria using the relationship between PDW and CDW wave-vectors above and below $T_{c}$. In the following chapter I will report on the use of scanned Josephson tunnelling microscopy (SJTM) a direct probe of the superconducting condensate in cuprates. Periodic modulations of the condensate are detected. Comparing their wavelength to those of the CDW reported on in chapter $4 \mathrm{I}$ will draw conclusions about the possible PDW scenaria in $\mathrm{Bi}_{2} \mathrm{Sr}_{2} \mathrm{CaCu}_{2} \mathrm{O}_{8+x}$. 



\section{Chapter 7}

\section{Detection of a Cooper-pair}

\section{Density Wave in}

\section{$\mathrm{Bi}_{2} \mathrm{Sr}_{2} \mathrm{CaCu}_{2} \mathrm{O}_{8+\delta}$}

The main results of this chapter are reported in "Direct Detection of a Cooper-pair Density Wave in $\mathrm{Bi}_{2} \mathrm{Sr}_{2} \mathrm{CaCu}_{2} \mathrm{O}_{8+\delta}$ ", Nature, 532, 343 (2016).

The quantum condensate of Cooper pairs forming a superconductor was originally conceived as being translationally invariant [8]. In theory, however, pairs can exist with non-zero momentum $\vec{Q}$, thus generating a state with a spatially modulated Cooper-pair density [61, 62]. Such a state has been created in ultracold ${ }^{6} \mathrm{Li}$ gas but never observed directly in any superconductor [172]. It is now widely hypothesised that the pseudogap phase of the cuprate superconductors contains such a "pair density wave" state $[103,167$, 168, 178, 179, 184, 195-197, 199, 201, 202, 207-211]. This chapter details the use of nanometre-resolution scanned Josephson tunnelling microscopy to image Cooper pair tunnelling from a $d$-wave superconducting STM tip to the condensate of the superconductor $\mathrm{Bi}_{2} \mathrm{Sr}_{2} \mathrm{CaCu}_{2} \mathrm{O}_{8+x}$. By using Fourier analysis of scanned Josephson tunnelling images, we discover the direct signature of a Cooper-pair density modulation. 


\subsection{Four Unit-Cell Periodic Modulations in the Cooper- Pair Current}

In this chapter I will report on the use of cuprate scanned Josephson tunnelling microscopy (SJTM), whose development is detailed in chapter 5, to study $\mathrm{Bi}_{2} \mathrm{Sr}_{2} \mathrm{CaCu}_{2} \mathrm{O}_{8+x}$ samples. This technique directly probes the superconducting condensate, through the tunnelling of Cooper-pairs, by forming a nanometer-resolution scannable Josephson junction between a superconducting STM tip and the sample. In our approach to cuprate SJTM this superconducting STM tip is formed by picking up a nanoflake of our $\mathrm{Bi}_{2} \mathrm{Sr}_{2} \mathrm{CaCu}_{2} \mathrm{O}_{8+x}$ sample with a normal tungsten STM tip. In operation, a maximum Cooper-pair current $I_{c}(\vec{r})$ between the tip and sample is measured at a fine grid of points. This allows one to map spatial variations of the superconducting condensate at the nanometer scale.

I will present results for $\mathrm{Bi}_{2} \mathrm{Sr}_{2} \mathrm{CaCu}_{2} \mathrm{O}_{8+x}$ samples with $T_{c}=88 \mathrm{~K}$ and hole density $p=17 \%$ at $T=45 \mathrm{mK}$. The two states already reported to coexist at this $p$ value are the high-temperature $d$-wave superconductor and a CDW with $\vec{Q}=(0.22 \pm 0.02,0) \frac{2 \pi}{a_{0}}$ and $(0,0.22 \pm 0.02) \frac{2 \pi}{a_{0}}$ (see chapter 4$)$. Figure 7.1 shows the measured $I_{c}(\vec{r})$ in a $76 \mathrm{~nm} \times$ $76 \mathrm{~nm}$ field of view. A $35 \times 35 \mathrm{~nm}$ portion of this image (dashed box) is shown enlarged in figure $7.2(\mathrm{a})$. Clear $I_{c}(\vec{r})$ modulations are immediately visible in both.

The magnitude of the Fourier transform of $I_{c}(\vec{r}),\left|\tilde{I}_{c}(\vec{q})\right|$, is shown in figure 7.2 (b) revealing that modulations in $I_{c}(\vec{r})$ occur at the wave-vectors $\vec{Q}_{P}=(0.25 \pm 0.02,0) \frac{2 \pi}{a_{0}}$ and $(0,0.25 \pm 0.02) \frac{2 \pi}{a_{0}}$ (dashed red circles in figure $\left.7.2(\mathrm{~b})\right)$. No peak at $\vec{q} \approx(0.125,0) \frac{2 \pi}{a_{0}}$ or $(0,0.125) \frac{2 \pi}{a_{0}}$ is observed above the background.

In figure $7.2(\mathrm{c})$ I show (as blue dots) the measured value of $I_{c}(\vec{r})$ along the blue line in figure 7.2 (a). The average amplitude and wavelength of the $I_{c}(\vec{r})$ modulations (determined from the magnitude and central $\vec{q}$ value of the peaks in figure $7.2(\mathrm{c}))$ are shown as the red line. From these data, and in general from the magnitude of the peaks at $\vec{Q}_{x}$ and $\vec{Q}_{x}$ in figure $7.2(\mathrm{~b})$, we conclude that the modulation amplitude in $I_{c}(\vec{r})$ is $\sim 5 \%$ of its average value. Therefore, at this doping $(p=17 \%)$, the modulation represents a small modulation on top of the uniform component, at least deep within the superconducting state $\left(T<<T_{c}\right)$ where these measurements were taken. 


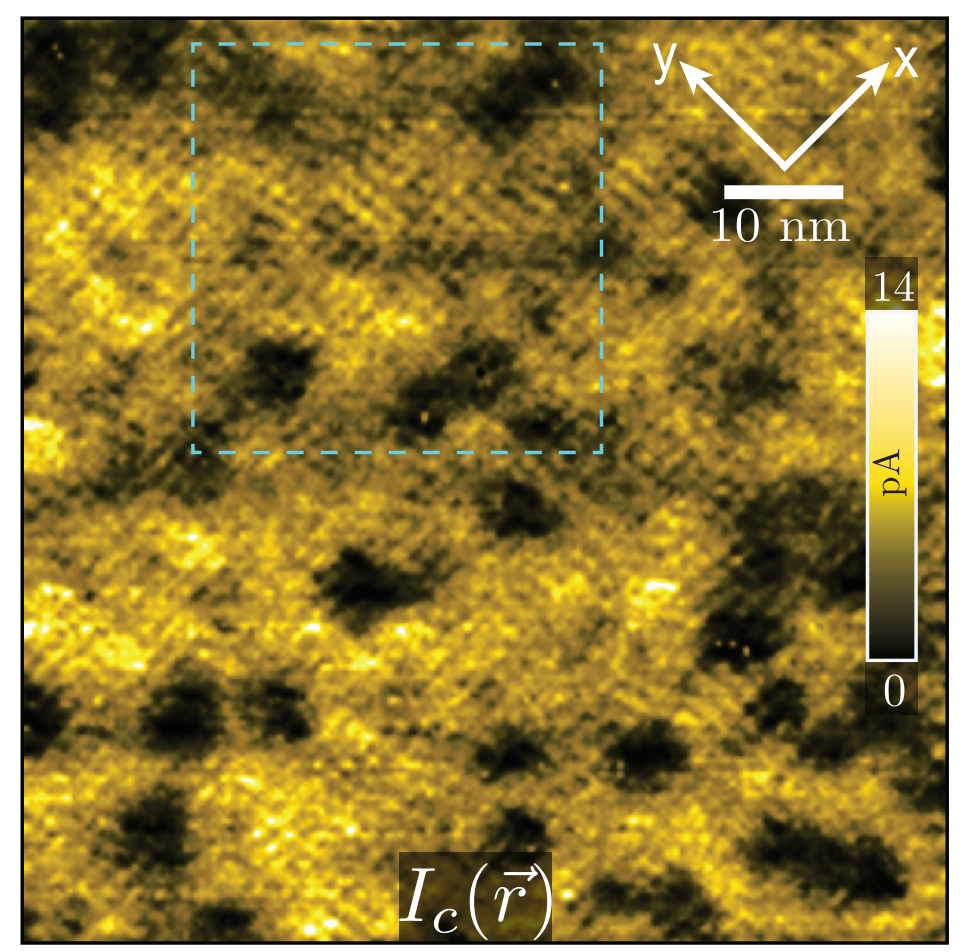

FiguRE 7.1: A $76 \mathrm{~nm} \times 76 \mathrm{~nm} I_{c}(\vec{r})$ image measured at $45 \mathrm{mK}$. For visual clarity modulations at the wave-vector of the crystal super-modulation have been removed by the Fourier filtration method described in section 2.8.

Now I turn to the relationship of the $I_{c}(\vec{r})$ modulations the known $d$-symmetry form factor CDW modulations in this material (see chapter 4). Figure 7.2 (d) shows $\tilde{D}(\vec{q})=$ $\tilde{O}_{x}(\vec{q})-\tilde{O}_{y}(\vec{q})$, for a $\mathrm{Bi}_{2} \mathrm{Sr}_{2} \mathrm{CaCu}_{2} \mathrm{O}_{8+x}$ sample with the same hole doping density and $T=2 \mathrm{~K}$. This can be regarded as a Fourier transform that measures the $d$-symmetry from factor component of modulations. The locations of $d$-symmetry form factor CDW peaks in $\tilde{D}(\vec{q})$ (dashed red circles) occur at $\vec{Q}_{C}=(0.22,0) \frac{2 \pi}{a_{0}} ;(0,0.22) \frac{2 \pi}{a_{0}}$.

In the unprocessed data the modulations in electronic structure and topography occur based upon the sum of both oxygen sub-lattices $\left(\tilde{O}_{x}(\vec{q})+\tilde{O}_{y}(\vec{q})\right)$ and at wave-vector $\vec{Q}=\vec{Q}_{\text {Bragg }} \pm \vec{Q}_{C}$. No response of the tip to these modulations could produce a spurious $I_{c}(\vec{r})$ modulation at the wave-vector $\vec{Q}_{P}=(0.25 \pm 0.02,0) \frac{2 \pi}{a_{0}}$ and $(0,0.25 \pm 0.02) \frac{2 \pi}{a_{0}}$. Further, no significant modulations occur in the normal state resistance, $R_{N}(\vec{q})$, at $\vec{Q}_{P}$ indicating that these modulations are not the result of a variations in the tip-sample distance (see appendix $\mathrm{H}$ ).

Obviously, figures 7.2 (b) and (d) are not identical, with $\left|\tilde{I}_{c}(\vec{q})\right|$ exhibiting much narrower peaks, implying a longer spatial coherence length for $I_{c}(\vec{r})$ modulations. Nevertheless, 
(a)

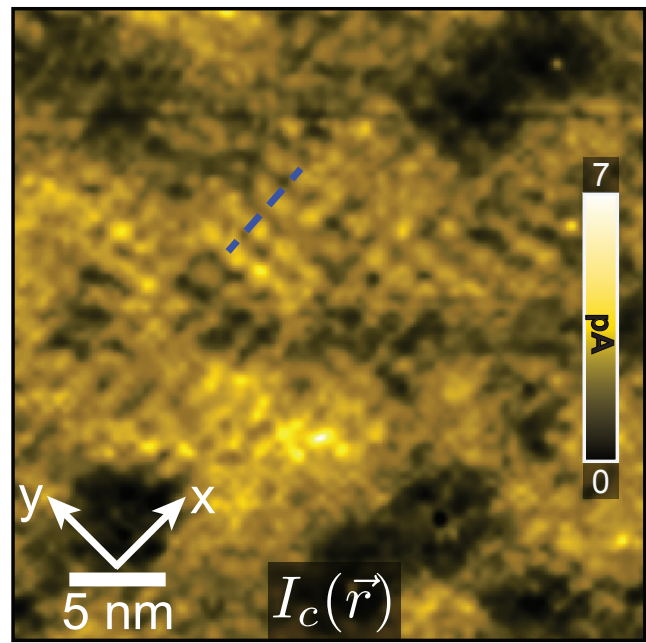

(c)

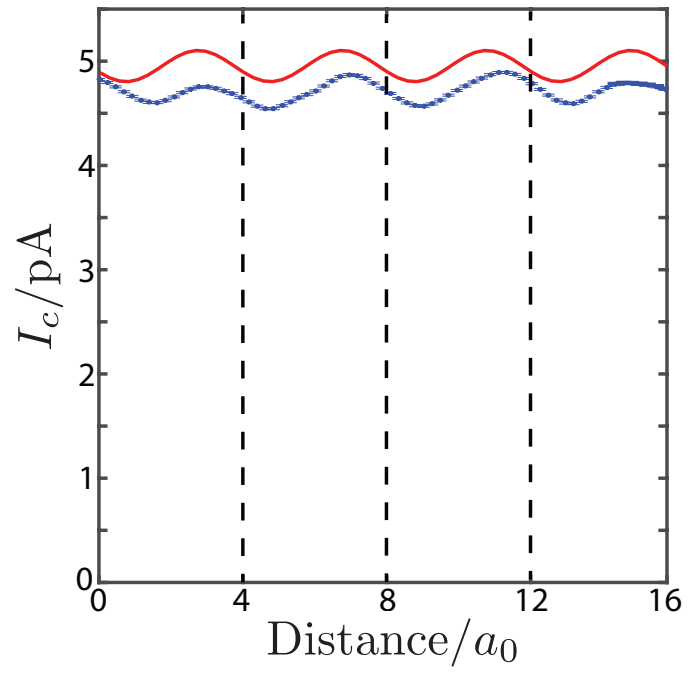

(b)

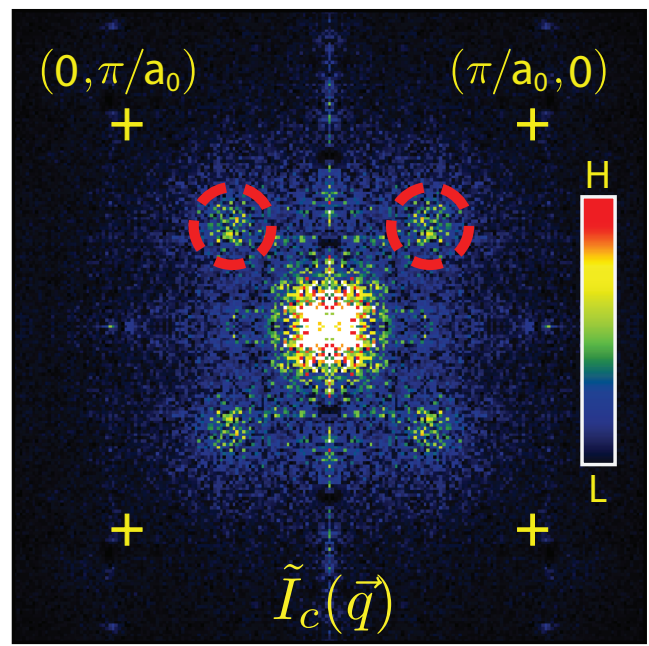

(d)

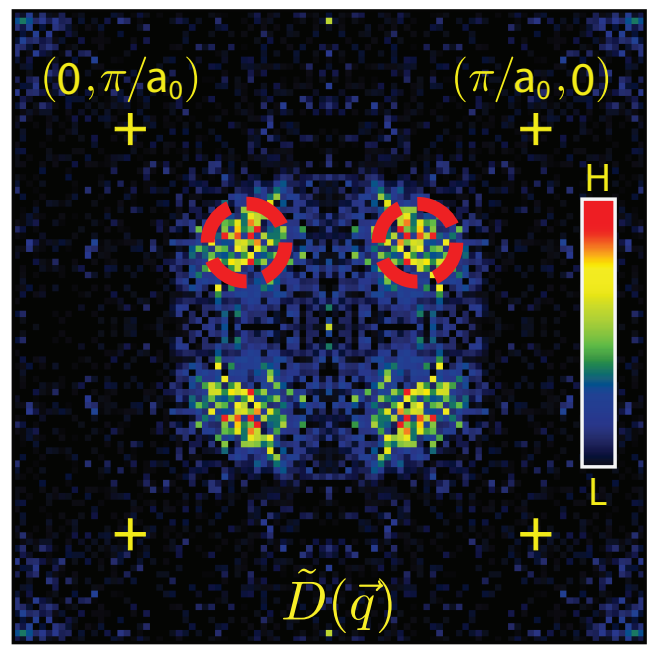

Figure 7.2: (a) A $35 \mathrm{~nm} \times 35 \mathrm{~nm}$ field of view $I_{c}(\vec{r})$ image (dashed blue box in figure 7.1) showing $I_{c}(\vec{r})$ modulations parallel to the $\mathrm{CuO}_{2} \mathrm{x}$ and y axes (supermodulationinduced $I_{c}(\vec{r})$ modulations are removed). (b) $\left|\tilde{I}_{c}(\vec{q})\right|$, the Fourier transform of $I_{c}(\vec{r})$ in (a) (yellow crosses at $\vec{q}=\left(\pi / \mathrm{a}_{0}, 0\right)$ and $\left(0, \pi / \mathrm{a}_{0}\right)$ ). Maxima from $I_{c}(\vec{r})$ modulations (dashed red circles) occur at $\vec{Q}_{P}=(0.25 \pm 0.02,0) \frac{2 \pi}{a_{0}}$ and $(0,0.25 \pm 0.02) \frac{2 \pi}{a_{0}}$. No significant modulations occur in $R_{N}(\vec{q})$ at $\vec{Q}_{P}$ (see appendix H). (c) Measured $I_{c}(\vec{r})$ along the dashed blue line in (a) (blue dotted line); statistical error bars are the variance of $I_{c}(\vec{r})$ transverse to the dashed line. The red line shows the global amplitude and $\vec{Q}_{P}$ of the $I_{c}(\vec{r})$ modulations (from the circled maxima in (b). (d) Magnitude of $\tilde{D}(\vec{q})=\tilde{O}_{x}(\vec{q})-\tilde{O}_{y}(\vec{q})$ (yellow crosses at $\vec{q}=\left(\pi / \mathrm{a}_{0}, 0\right)$ and $\left.\left(0, \pi / \mathrm{a}_{0}\right)\right)$ revealing the $d$-symmetry form factor density wave modulations (dashed red circles) at $\vec{Q}_{C}=(0.22,0) \frac{2 \pi}{a_{0}} ;(0,0.22) \frac{2 \pi}{a_{0}}$ for a sample with the same hole doping as in (b). This Fourier transform is symmetrised about the vertical axis. 
the $I_{c}(\vec{r})$ modulation wave-vector, $\vec{Q}_{P}$, is consistent (within joint error bars) with the wave-vector of the CDW at the same hole doping.

The $I_{c}(\vec{r})$ modulations also exhibit a primarily $s / s^{\prime}$-symmetry form factor. One deduces this because figure 7.2 (b) is a conventional Fourier transform based on the sum of all sub-lattices. This is in contrast to the CDW modulations, which exhibits a primarily $d$-symmetry form factor. Again, no peaks in $\left|\tilde{I}_{c}(\vec{q})\right|$ at $\vec{Q} \approx \vec{Q}_{C} / 2$ are observed above the background. However, if modulations at $\vec{Q} \approx \vec{Q}_{C} / 2$ existed with a $d$-symmetry form factor, our SJTM would likely not resolve them due to lack of intra-unit-cell resolution.

Having demonstrated in chapter 5 that our SJTM faithfully probes variations in the superfluid density, the results presented above provide strong, direct evidence for an approximately four unit-cell periodic modulation of the superconducting condensate in $\mathrm{Bi}_{2} \mathrm{Sr}_{2} \mathrm{CaCu}_{2} \mathrm{O}_{8+x}$. Furthermore, we have demonstrated that this modulation has approximately the same wave-vector as the known predominantly $d$-symmetry form factor CDW in this material at the same doping. In the following section I will discuss these $I_{c}(\vec{r})$ modulations in the context of a PDW component to the superconducting condensate.

\subsection{Discussion of $I_{c}(\vec{r})$ Modulations as a Pair Density Wave}

In chapter 5 I demonstrated that the maximum Cooper-pair current, $I_{c}(\vec{r})$, faithfully measures spatial variations in the superfluid density. In light of this, we ascribe the periodic $I_{c}(\vec{r})$ modulations described above as being due to a pair density wave (PDW) component in the superconducting condensate.

As discussed in chapter 5 , the intrinsic Josephson critical current, $I_{J}$, is proportional to $\left|\Psi_{0}\right| . \Psi_{0}$ is the Ginzburg-Landau order parameter for a uniform superconductor, related to the superfluid density, $\rho_{s}$, through $\rho_{s}=\frac{\hbar^{2}}{2 m}\left|\Psi_{0}\right|^{2}$. As detailed in chapter 5 , the ultrasmall junction in our SJTM supports a maximum Cooper pair current $I_{c} \propto I_{J}^{2}$, which is reduced in magnitude as compared to $I_{J}$ by phase fluctuations across the junction. Thus, measurements of $I_{c}$ are proportional to $\left|\Psi_{0}\right|^{2}$.

To explore the consequences of a PDW for our SJTM experiment we model our tip as point-like, with a uniform superconducting order parameter that couples to a modulating 
order parameter in the sample

$$
\Psi(\vec{r})=\Psi_{0}+\Psi_{\vec{Q}_{P}} e^{i \vec{Q}_{P} \cdot \vec{r}}+\Psi_{-\vec{Q}_{P}} e^{-i \vec{Q}_{P} \cdot \vec{r}}+\ldots
$$

The modulation is superposed on top of a uniform superconducting component because our measurements are made deep within the superconducting phase $\left(T=45 \mathrm{mK}, T_{c}=\right.$ $88 \mathrm{~K})$. This will give a corresponding modulation in the intrinsic Josephson critical current $I_{J}=I_{J}^{0}+I_{P} \cos (\vec{Q} \cdot \vec{r})$. The maximum Cooper-pair current, $I_{c} \propto I_{J}^{2}$, will then modulate as

$$
I_{c} \propto\left(I_{J}^{0}\right)^{2}+2 I_{J}^{0} I_{J}^{P} \cos \left(\vec{Q}_{P} \cdot \vec{r}\right)+\left(I_{J}^{P}\right)^{2} \cos ^{2}(\vec{Q} \cdot \vec{r})+\ldots
$$

The PDW $\Psi_{\vec{Q}_{P}}$ will manifest itself as a modulation at wave-vector $\vec{Q}_{P}$ on top of a background corresponding to the uniform $d$-wave superconductor.

One could also take the opposite point of view and treat the tip as though it were a piece of $\mathrm{Bi}_{2} \mathrm{Sr}_{2} \mathrm{CaCu}_{2} \mathrm{O}_{8+x}$, with large extent in the $x$ and $y$ directions, having both a uniform and PDW superconducting component. This is potentially a valid model because the spatial extent of the tip is of the same order of magnitude as the $I_{c}(\vec{r})$ modulation wavelength. The Josephson coupling between tip and sample is then roughly equivalent to the inter-layer Josephson coupling intrinsic to the material, with a spatial phase shift between the PDW in the two layers that accounts for the position of the tip over the surface.

In this case, one would also expect a PDW with wave-vector $\vec{Q}_{P}$ to modulate the intrinsic Josephson critical current as $I_{J}=I_{J}^{0}+I_{P} \cos (\vec{Q} \cdot \vec{r})$. This would lead to the same form of $I_{c}(\vec{r})$ given in equation 7.2. Again, the PDW $\Psi_{\vec{Q}_{P}}$ would manifest itself in $I_{c}(\vec{r})$ as a modulation at wave-vector $\vec{Q}_{P}$ on top of a background corresponding to the uniform $d$-wave superconductor.

The data presented in the previous section are clear experimental evidence for modulations of the superconducting condensate in $\mathrm{Bi}_{2} \mathrm{Sr}_{2} \mathrm{CaCu}_{2} \mathrm{O}_{8+x}$ with wave-vector $\vec{Q}_{P}=(0.25 \pm 0.02,0) \frac{2 \pi}{a_{0}}$. The simple models of the tip-sample Josephson coupling given in this section demonstrate that this can be viewed as a PDW component coexisting with a robust uniform condensate. 


\subsection{Conclusions}

The visualisation of a PDW, a long-term challenge in physics has now been achieved using SJTM. This represents the first direct detection of a periodically modulating superconducting pair-amplitude, akin to the FFLO phase first predicted in 1964. The PDW is observed in $\mathrm{Bi}_{2} \mathrm{Sr}_{2} \mathrm{CaCu}_{2} \mathrm{O}_{8+x}$ at $17 \%$ hole doping, coexisting with the $d$-wave high-temperature superconductor. The PDW gives rise to modulations in $I_{c}(\vec{r})$ whose amplitude is $\sim 5 \%$ that due to the $d$-wave superconductor. The PDW has approximately the same wave-vector as the $d$-symmetry form factor CDW $\left(\vec{Q}_{P} \approx \vec{Q}_{C}\right)$ that exists at the same doping in this material albeit with an $s / s^{\prime}$-symmetry form factor.

That a PDW is detected at $\vec{Q}_{P} \approx \vec{Q}_{C}$ with a relatively small amplitude compared to the $d$-wave superconducting component is consistent with a PDW arising from the coupling of the CDW to the $d$-wave superconductor (scenario 3 in table 6.2). This follows naturally from the Ginzburg-Landau analysis in section 6.4 (specifically equation 6.13). Furthermore, that no PDW modulations are detected at $\vec{Q} \approx \vec{Q}_{C} / 2$ is seemingly inconsistent with the idea that the CDW should be regarded as a secondary order parameter generated through the product of primary PDW order parameters (scenario 1 in table 6.2 ). However, given that our SJTM may not be sensitive to modulations at $\vec{Q} \approx \vec{Q}_{C} / 2$ if they have a $d$-symmetry form factor, we should not draw that conclusion based on SJTM alone.

If a PDW with wave-vector $\vec{Q}_{P} \approx \vec{Q}_{C} / 2$ co-exists with the $d$-symmetry superconductor it should, in addition the the CDW at $\vec{Q}_{C}$ present above $T_{c}$, induce a CDW at $\vec{Q}=\vec{Q}_{C} / 2$ for $T<T_{c}$. No CDW at this wave-vector has been reported in $\mathrm{Bi}_{2} \mathrm{Sr}_{2} \mathrm{CaCu}_{2} \mathrm{O}_{8+x}$ either by STM or scattering probes. This in turn suggests that if a PDW with $\vec{Q}_{C} / 2$ exists in $\mathrm{Bi}_{2} \mathrm{Sr}_{2} \mathrm{CaCu}_{2} \mathrm{O}_{8+x}$ it must be dynamical.

As well as being consistent with a scenario in which primary CDW and $d$-wave superconducting order parameters couple to form a PDW for $T<T_{c}$, our results would also be consistent with models where CDW and PDW (both primary) co-exist at the same $|\vec{Q}|$ (scenario 3 in table 6.2). Through equation 6.9 this should produce a CDW at wavevector $2 \vec{Q}_{C}$, but it is highly plausible that this modulation is too small to detect with present experimental capabilities. 
This work is only the first foray into direct, nano-resolved, measurements of the pairing amplitude in cuprate superconductors. One would like to search for the existence of a PDW above the bulk $T_{c}$, where $\Psi_{0}=0, \Psi_{\vec{Q}} \neq 0$. This would constitute the detection of a truly new state of matter $[168,179]$. To search for this with SJTM the best candidate material would be $\mathrm{La}_{2-x} \mathrm{Ba}_{x} \mathrm{CuO}_{4}$ at $1 / 8$ th doping. Here, the bulk $T_{c}$ is small enough that SJTM could feasibly operate above it. Additionally, there is a good deal of indirect evidence suggesting that such a state does indeed exist here (see section 6.2). Finally, our SJTM approach to nanometre-resolution Cooper-pair condensate imaging also reveals a direct route to visualisation of FFLO or PDW states in other cuprates, iron-pnictides and unconventional superconductors. 


\section{Chapter 8}

\section{Summary and Future Directions}

In this thesis I have presented research that, through the introduction of two novel techniques, examines the nature of density wave order in cuprates and its relationship to superconductivity.

In chapter 3 I built upon recent advances in STM visualisation of intra unit cell electronic structure to introduce sub-lattice segregated spectroscopic-imaging STM (SI-STM) in cuprates, whereby the atomic sub-lattices of the $\mathrm{CuO}_{2}$ plane can be individually addressed. In chapter $4 \mathrm{I}$ went on to use this technique to reveal that the short-ranged charge density wave (CDW) in $\mathrm{Bi}_{2} \mathrm{Sr}_{2} \mathrm{CaCu}_{2} \mathrm{O}_{8+\delta}$ and $\mathrm{Na}_{x} \mathrm{Ca}_{2-x} \mathrm{CuO}_{2} \mathrm{Cl}_{2}$ has a predominantly $d$-symmetry form factor. In such a CDW, a breaking of rotational symmetry within the unit is modulated periodically in space, giving the CDW a local $d$-wave character. Although its existence has been predicted for many years, this is the first observation of a $d$-symmetry form factor CDW in nature [90-106].

It is natural to ask whether there is any profound connection between the $d$-wave nature of the CDW form factor and the well known $d$-wave superconductivity in cuprates. Within itinerant spin-fermion "hot-spot" models of the CDW and superconductivity in cuprates the connection is indeed a deep one [94-97, 101, 103, 104]. Here it arises because both $d$-symmetry form factor CDW and $d$-wave superconductivity emerge from the same effective interaction between fermions at specific points in momentum space. If these models are indeed a correct description of the superconductivity and CDW in cuprates, the existence of a predominantly $d$-symmetry form factor CDW cannot be disentangled from that of the high-temperature $d$-wave superconductivity. 
However, our measurements do not clearly distinguish between different models of CDW formation in cuprates that exhibit a predominantly $d$-symmetry form factor. For instance, it has also been proposed that the $d$-symmetry form factor results from the Coulomb repulsion between holes on the two oxygen sites in the $\mathrm{CuO}_{2}$ plaquette $[98$, $102,105,106]$. This favours unequal occupation of these sites, naturally resulting in a local $d$-wave character to the CDW.

The observation of a short ranged $\mathrm{CDW}$ in the electron doped cuprate $\mathrm{Nd}_{2-x} \mathrm{Ce}_{x} \mathrm{CuO}_{4}$, where carriers are first doped into bands derived from the $\mathrm{Cu} 3 \mathrm{~d}_{x^{2}-y^{2}}$ orbitals, provides a way to falsify this hypothesis [131]. If measurements sensitive to the CDW form factor reveal that it is predominantly $d$-wave in electron doped cuprates, the hypothesis cannot be true.

Independent of a specific model of CDW formation in cuprates, a $d$-symmetry form factor $\mathrm{CDW}$ is a natural partner to $d$-wave superconductivity in the sense that it acts to protect the coherence of nodal quasi-particles against scattering from the disordered CDW in so called "cold-spots" [118]. That the $\mathrm{CDW}$ in $\mathrm{La}_{2-x} \mathrm{Ba}_{x} \mathrm{CuO}_{4+\delta}$ has a predominantly $s$-symmetry form factor, in contrast to other cuprates materials, raises the question of whether its anomalously large suppression of $T_{c}$ at $1 / 8$ th doping can be attributed to the form factor of its CDW [116].

I have also demonstrated that the charge order in $\mathrm{Bi}_{2} \mathrm{Sr}_{2} \mathrm{CaCu}_{2} \mathrm{O}_{8+\delta}$ and $\mathrm{Na}_{x} \mathrm{Ca}_{2-x} \mathrm{CuO}_{2} \mathrm{Cl}_{2}$ consists of nanoscale domains of unidirectional $d$-symmetry form factor CDW. Recent high-magnetic field measurements in $\mathrm{YBa}_{2} \mathrm{Cu}_{3} \mathrm{O}_{6+x}$ reveal a magneticfield induced CDW that is completely unidirectional $[55,56]$. This is suggestive that the $\mathrm{CuO}_{2}$ plane has an intrinsic instability to unidirectional ordering. A cautionary caveat is that $\mathrm{YBa}_{2} \mathrm{Cu}_{3} \mathrm{O}_{6+x}$ is structurally orthorhombic, and so possesses no symmetry between $\mathrm{Cu}-\mathrm{O}$ directions. I propose that measurements of magnetic field induced CDW in cuprates with a structural symmetry between the $\mathrm{Cu}-\mathrm{O}$ directions be performed with the aim of directly demonstrating this unidirectional tendency of the $\mathrm{CuO}_{2}$ plane.

In chapter 5 I detailed the development of nanometer resolution scanned Josephson tunnelling microscopy (SJTM). By measuring the Cooper pair (Josephson) tunnelling current between a superconducting STM tip and a superconducting sample it was possible to directly visualise the superconducting condensate with nanometer resolution for the first time. 
Utilising SJTM, the visualisation of a pair density wave (PDW), a long-term challenge in physics, has now been achieved. This represents the first direct detection of a periodically modulating superconducting condensate, akin to the FFLO phase first predicted in 1964. The PDW is observed in $\mathrm{Bi}_{2} \mathrm{Sr}_{2} \mathrm{CaCu}_{2} \mathrm{O}_{8+x}$ at $17 \%$ hole concentration, coexisting with the uniform $d$-wave high-temperature superconductivity. The PDW gives rise to modulations in the Cooper pair tunnelling current whose amplitude is $\sim 5 \%$ of that due to the uniform $d$-wave superconducting component. It has approximately the same wave-vector as the $d$-symmetry form factor CDW that exists at the same doping in this material, albeit with an $s / s^{\prime}$-symmetry form factor.

By comparing the observed PDW wave-vector to that of the CDW above and below $T_{c}$, I conclude that the PDW observed in SJTM is either the result of coupling between the CDW and uniform superconductivity, or an instability of the $\mathrm{CuO}_{2}$ plane to both PDW and CDW order at the same wave-vector. This is in contrast with the possibility that a PDW induces a CDW at twice its fundamental wave-vector. A route toward distinguishing between the two remaining scenaria would be to extend our SJTM measurements to cover a wider range of dopings. Additionally, if we could attain intra unit cell resolution cuprate SJTM we could directly examine the spatial relationship between CDW and PDW, thus determining whether they are independent or not.

Beyond the cuprates, SJTM shows great promise as a direct nano-resolved probe of superconducting condensates. As discussed in chapter 5, the local single-particle tunnelling gap of a superconductor is not easily related to the local superfluid stiffness or superconducting order parameter magnitude in all but a few cases. In contrast, SJTM measures the local superfluid stiffness in a way that is independent of any microscopic model of superconductivity.

SJTM has the potential to be a formidable tool in the study of any system where the nanoscale heterogeneity of the superconducting condensate is of interest. This includes superconducting devices and heterostructures, materials where superconductivity is intertwined with orders which break translational symmetry and the study of the effect of impurities on superconductivity.

SJTM could be of considerable use in the study of how to increase the critical current of superconductors through vortex pinning, currently of great technological interest. Because it can be operated in either single-particle or Cooper pair tunnelling modes, 
SJTM offers the ability to image the nature of defects, both natural and artificial, whilst simultaneously visualising the local superfluid stiffness. This could provide significant advances in our understanding of the vortex pinning processes in technologically relevant materials.

Finally, as detailed in chapter 5, SJTM utilises the inelastic tunnelling of Cooper-pairs to access the superconducting condensate. This is the simplest form of inelastic tunnelling because the Cooper-pair condensate does not form a continuum of initial and final states. The emission and absorption of electromagnetic radiation in these processes allows for the possibility of nano-resolved spectroscopy or manipulation of adatoms on a superconducting surface. 


\section{Appendix A}

\section{Publications Arising from Doctoral Studies}

Below I list publications arising from my doctoral studies, grouped by subject matter.

\section{A.1 Piezoelectric Strain Tuning}

C. W. Hicks, D. O. Brodsky, E. A. Yelland, A. S. Gibbs, J. A. N. Bruin, M. B. Barber, S. D. Edkins, K. Nishimura, S. Yonezawa, Y. Maeno, A. P. Mackenzie. "Strong Increase of $\mathrm{T}_{c}$ of $\mathrm{Sr}_{2} \mathrm{RuO}_{4}$ Under Both Tensile and Compressive Strain". Science 344 283-285 (2014)

C. W. Hicks, M. E. Barber, S. D. Edkins, D. O. Brodsky, A. P. Mackenzie. "Piezoelectricbased apparatus for strain tuning". Review of Scientific Instruments 85(6) 065003 (2014).

\section{A.2 Spectroscopic Imaging STM of the Cuprate CDW}

K. Fujita, M. H. Hamidian, S. D. Edkins, Chung Koo Kim, Y. Kohsaka, M. Azuma, M. Takano, H. Takagi, H. Eisaki, S. Uchida, A. Allais, M. J. Lawler, E. -A. Kim, S. Sachdev,

J. C. Davis. "Direct phase-sensitive identification of a d-form factor density wave in 
underdoped cuprates". Proceedings of the National Academy of Sciences 111(30) E3026E3032 (2014).

M. H. Hamidian*, S. D. Edkins*, Chung Koo Kim, JC Davis, AP Mackenzie, H Eisaki, S Uchida, MJ Lawler, E-A Kim, Subir Sachdev, K Fujita. "Atomic-scale electronic structure of the cuprate d-symmetry form factor density wave state". Nature Physics 12150 (2016).

A. Mesaros, K. Fujita, S. D. Edkins, M. H. Hamidian, H. Eisaki, S. Uchida, J. C. Samus Davis, M. J. Lawler, Eun-Ah Kim. "Commensurate $4 \mathrm{a}_{0}$ period Charge Density Modulations throughout the $\mathrm{Bi}_{2} \mathrm{Sr}_{2} \mathrm{CaCu}_{2} \mathrm{O}_{8+x}$ Pseudogap Regime" PNAS (2016) doi:10.1073/pnas.1614247113.

\section{A.3 Scanned Josephson Tunnelling Microscopy}

M. H. Hamidian*, S. D. Edkins*, Sang Hyun Joo*, A. Kostin, H. Eisaki, S. Uchida, M. J. Lawler, E. -A. Kim, A. P. Mackenzie, K. Fujita, Jinho Lee, J. C. Samus Davis. "Detection of a Cooper-pair density wave in $\mathrm{Bi}_{2} \mathrm{Sr}_{2} \mathrm{CaCu}_{2} \mathrm{O}_{8+x}$ ". Nature 532343 (2016).

* These authors contributed equally to this publication. 


\section{Appendix B}

\section{A Form Factor Description of Local Rotational Symmetry in}

\section{Density Waves}

In classifying the local rotational symmetry of density waves there is an ambiguity that arises because the wave-vector already breaks a global rotation symmetry itself. This leads to multiple form factor descriptions of the same state. Here I will adopt the convention introduced by Sachdev and La Placa [99]. Working in a one-site representation of the $\mathrm{CuO}_{2}$ plane with copper sites at positions $\vec{r}_{i}$ and $\vec{r}_{j}$ one can parameterise a CDW order via,

$$
\left\langle\hat{c}_{i \sigma}^{\dagger} \hat{c}_{j \sigma}\right\rangle=\sum_{\vec{Q}}\left[\sum_{\vec{k}} P(\vec{k}, \vec{Q}) e^{i \vec{k} \cdot\left(\vec{r}_{i}-\vec{r}_{j}\right)}\right] e^{i \vec{Q} \cdot\left(\vec{r}_{i}+\vec{r}_{j}\right) / 2}
$$

where $\hat{c}_{j \sigma}$ annihilates a fermion with spin $\sigma$ on a site at position $\vec{r}_{i}$. Here the wave vector $\vec{Q}$ is associated with a modulation in the average coordinate $\left(\vec{r}_{i}+\vec{r}_{j}\right) / 2$. The form factor describes the dependence on the relative co-ordinate $\vec{r}_{i}-\vec{r}_{i}$. An advantage of the formulation in equation B.1 is that it provides an efficient characterisation of symmetries. The operator identity $\left\langle\hat{A}^{\dagger}\right\rangle \equiv\langle\hat{A}\rangle^{*}$ requires that

$$
P^{*}(\vec{k}, \vec{Q})=P(\vec{k},-\vec{Q})
$$

and

$$
P(\vec{k}, \vec{Q})=P(-\vec{k}, \vec{Q})
$$


if time-reversal symmetry is preserved.

Here I will consider a $P(\vec{k}, \vec{Q})$ that is non-zero only for $\vec{Q}=\vec{Q}_{x}=(Q, 0)$ and $\vec{Q}=\vec{Q}_{y}=$ $(0, Q)$, the experimentally detected wave-vector in cuprates $[27,58,59]$. Assuming that time-reversal symmetry is preserved the form factor has the general form

$$
P(\vec{k}, \vec{Q})=P_{S}+P_{S^{\prime}}\left(\cos \left(k_{x}\right)+\cos \left(k_{y}\right)\right)+P_{D}\left(\cos \left(k_{x}\right)-\cos \left(k_{y}\right)\right)+\ldots
$$

For general incommensurate $\vec{Q}$, any even function of $\vec{k}$ is allowed on the right-hand side. The case of a predominantly $d$-symmetry form factor CDW considered in chapter 4 is parameterised by $\left|P_{D}\right| \gg\left|P_{S^{\prime}}\right|$ and $\left|P_{D}\right| \gg\left|P_{S}\right|$

We can now make a connection between this abstract parameterisation of the intra-unitcell rotational symmetry of the CDW and the local observables measured using STM. Making a transformation from the three-site to the single-site model of the $\mathrm{CuO}_{2}$ layer, we can deduce the relationship

$$
\left\langle\hat{c}_{i \sigma}^{\dagger} \hat{c}_{j \sigma}+\hat{c}_{j \sigma}^{\dagger} \hat{c}_{i \sigma}\right\rangle \begin{cases}\frac{1}{K} \rho\left(\vec{r}_{C u}\right) & \text { for } i=j \\ \frac{1}{K^{\prime}} \rho\left(\vec{r}_{O_{x}}\right) & \text { for } i, j \text { n.n along x direction } \\ \frac{1}{K^{\prime}} \rho\left(\vec{r}_{O_{y}}\right) & \text { for } i, j \text { n.n along y direction. }\end{cases}
$$

Here $\rho(\vec{r})$ is any density-like observable (i.e., invariant under time-reversal and spin rotations) such as charge or local density of states and $K$ and $K^{\prime}$ are constants. Combining equations B.1, B.4 and B.5 we can now write

$$
\begin{gathered}
\rho\left(\vec{r}_{C u}\right)=2 K \operatorname{Re}\left\{\left[\sum_{\vec{k}} P(\vec{k}, \vec{Q})\right] e^{i \vec{Q} \cdot \vec{r}_{C u}}\right\} \\
=A_{s} \cos \left(\vec{Q} \cdot \vec{r}_{C u}+\phi_{s}\right) \\
\rho\left(\vec{r}_{O_{x}}\right)=2 K^{\prime} \operatorname{Re}\left\{\left[\sum_{\vec{k}} \cos \left(k_{x}\right) P(\vec{k}, \vec{Q})\right] e^{i \vec{Q} \cdot \vec{r}_{O_{x}}}\right\} \\
=A_{s^{\prime}} \cos \left(\vec{Q} \cdot \vec{r}_{O_{x}}+\phi_{s^{\prime}}\right)+A_{D} \cos \left(\vec{Q} \cdot \vec{r}_{O_{x}}+\phi_{D}\right) \\
\rho\left(\vec{r}_{O_{y}}\right)=2 K^{\prime} \operatorname{Re}\left\{\left[\sum_{\vec{k}} \cos \left(k_{y}\right) P(\vec{k}, \vec{Q})\right] e^{i \vec{Q} \cdot \vec{r}_{O_{y}}}\right\} \\
=A_{s^{\prime}} \cos \left(\vec{Q} \cdot \vec{r}_{O_{y}}+\phi_{s^{\prime}}\right)-A_{D} \cos \left(\vec{Q} \cdot \vec{r}_{O_{y}}+\phi_{D}\right)
\end{gathered}
$$


with $A_{S}=2 K\left|P_{S}\right|, A_{S^{\prime}, D}=2 K^{\prime}\left|P_{S^{\prime}, D}\right|$ and $\phi_{S, S^{\prime}, D}=\arg \left(P_{S, S^{\prime}, D}\right)$.

With the simplification $\phi_{S}=\phi_{S^{\prime}}=\phi_{D}=\phi(\vec{r})$ and allowing for spatial disorder of the phase of the CDW we arrive at

$$
\rho(\vec{r})=\left[S(\vec{r})+S^{\prime}(\vec{r})+D(\vec{r})\right] \cos (\vec{Q} \cdot \vec{r}+\phi(\vec{r})),
$$

with $S(\vec{r})=A_{S}$ if $\vec{r} \in \vec{r}_{C u}$ and otherwise zero; $S^{\prime}(\vec{r})=A_{S^{\prime}}$ if $\vec{r} \in \vec{r}_{O_{x}}$ or $\vec{r}_{O_{y}}$ and otherwise zero; $D(\vec{r})=A_{D}$ if $\vec{r} \in \vec{r}_{O_{x}},-A_{D}$ if $\vec{r} \in \vec{r}_{O_{y}}$ and otherwise zero. This is equation 4.7 in chapter 4 .

The case of a predominantly $d$-symmetry form factor CDW discussed in this chapter is parameterised by $A_{D} \gg A_{S}$ and $A_{D} \gg A_{S^{\prime}}$. By inspection of equations B.6 one can see that a purely $d$-symmetry form factor CDW exhibits density modulations only on the $\mathrm{O}_{x}$ and $\mathrm{O}_{y}$ sites, but with a $\pi$ phase shift between them, as presented in chapter 4 . 



\section{Appendix C}

\section{Calculating Sub-lattice Fourier}

\section{Transforms}

As discussed in chapter 4 , the projection of a CDW into $s^{-}, s^{\prime}-$, and $d$-symmetry form factor components is conceptually appealing. However, for the purposes of this appendix I will work in terms of the segregated oxygen sublattice images $O_{x}(\vec{r})$.

In terms of the segregated sub-lattices, a $d$-symmetry form factor CDW is one for which the CDW on the $\mathrm{O}_{x}$ sites is in anti-phase with that on the $\mathrm{O}_{y}$ sites. For $\vec{q} \neq 0$ ordering the form factor does not uniquely determine the point group symmetry of the CDW and hence in general $s^{-}, s^{\prime}-$, and $d$-symmetry form factors are free to mix. This appendix predicts the consequences of a primarily $d$-symmetry form factor CDW for $\tilde{O}_{x, y}(\vec{q})$ and shows its consistency with the data presented in chapter 4 .

To deduce the logical consequences of a $d$-symmetry form factor CDW for the Fourier transforms of the segregated oxygen site images one can start by constructing the dual real and momentum-space representation of the sub-lattices:

$$
\begin{gathered}
L_{C u}(\vec{r})=\sum_{i, j} \delta\left(\vec{r}-\vec{R}_{i, j}\right) \Leftrightarrow \tilde{L}_{C u}(\vec{q})=\sum_{h, k} \delta\left(\vec{q}-G^{h, k}\right) \\
L_{O_{x}}(\vec{r})=L_{C u}\left(\vec{r}-\frac{a_{0} \hat{\vec{x}}}{2}\right) \Leftrightarrow \tilde{L}_{O_{x}}(\vec{q})=e^{i \frac{a_{0} \hat{\hat{x}}}{2}} \tilde{L}_{C u}(\vec{q}) \\
L_{O_{y}}(\vec{r})=L_{C u}\left(\vec{r}-\frac{a_{0} \hat{\vec{y}}}{2}\right) \Leftrightarrow \tilde{L}_{O_{y}}(\vec{q})=e^{i \frac{a_{0} \hat{\hat{y}}}{2}} \tilde{L}_{C u}(\vec{q}) .
\end{gathered}
$$


The $\left\{R_{i, j}\right\}$ are the set of direct lattice vectors of the square lattice with lattice constant $a_{0}$ and the $G^{h, k}$ are the reciprocal lattice vectors. The displacement of the oxygen sub-lattices from the copper sub-lattice has the effect of modulating the phase of their Bragg peaks along the direction of displacement with periodicity $4 \pi / a_{0}$ in reciprocal space. This is depicted in figure C.1 (a).

Using the convolution theorem, a $d$-symmetry form factor modulation of the oxygen site density takes on the dual description

$$
\begin{gathered}
O_{x}(\vec{r})=L_{O_{x}}(\vec{r}) \cdot A_{O_{x}}(\vec{r}) \Leftrightarrow \tilde{O}_{x}(\vec{q}) \star \tilde{A}_{O_{x}}(\vec{q}) \\
O_{y}(\vec{r})=L_{O_{y}}(\vec{r}) \cdot A_{O_{y}}(\vec{r}) \Leftrightarrow \tilde{O}_{y}(\vec{q}) \star \tilde{A}_{O_{y}}(\vec{q}) \\
A_{O_{x}}(\vec{r})=-A_{O_{y}}(\vec{r})=A(\vec{r}) \Leftrightarrow \tilde{A}_{O_{x}}(\vec{q})=-\tilde{A}_{O_{y}}(\vec{q})=\tilde{A}(\vec{q}) .
\end{gathered}
$$

The functions $O_{x, y}(\vec{r})$ are the segregated oxygen sub-lattice images. The $A_{O_{x, y}}(\vec{r})$ are continuous functions that when multiplied by the sub-lattice functions yield density waves in anti-phase on the separate oxygen sub-lattices (figure C.1 (b)). Figure C.1 (c) shows their Fourier transforms $\tilde{A}_{O_{x . y}}(\vec{q})$. Note that $A(\vec{r})$ may contain arbitrary amplitude and overall phase disorder but maintain the $d$-symmetry form factor as long as the relative phase relation in equation C.6 is maintained.

As shown in figure C.2 (a), the convolutions in equations C.4 and C.5 create an image of $\tilde{A}_{O_{x \cdot y}}(\vec{q})$ at each reciprocal lattice vector that sums to form the total convolution. Labeling the convolution image due to the reciprocal lattice vector $(h, k)$ in the x sublattice $\tilde{O}_{x}^{h, k}(\vec{q})$,

$$
\tilde{O}_{x}(\vec{q})=\sum_{h, k} \tilde{O}_{x}^{h, k}(\vec{q})=\sum_{h, k} e^{i\left(\vec{G}^{h, k}-\frac{a_{0} \hat{\vec{x}}}{2}\right)} \tilde{A}_{O_{x}}\left(\vec{q}-\vec{G}^{h, k}\right)
$$

In creating the $(h, k)$ convolution image, the phase of the sub-lattice Bragg peak at $\vec{G}^{h, k}$ and that of $\tilde{A}_{O_{x}}(\vec{q})$ must be added:

$$
\arg \left\{\tilde{O}_{x}^{h, k}(\vec{q})\right\}=\arg \left\{\tilde{A}_{O_{x}}\left(\vec{q}-\vec{G}^{h, k}\right)\right\}+\arg \left\{e^{i\left(\vec{G}^{h, k}-\frac{a_{0} \hat{\vec{x}}}{2}\right)}\right\}
$$


(a)

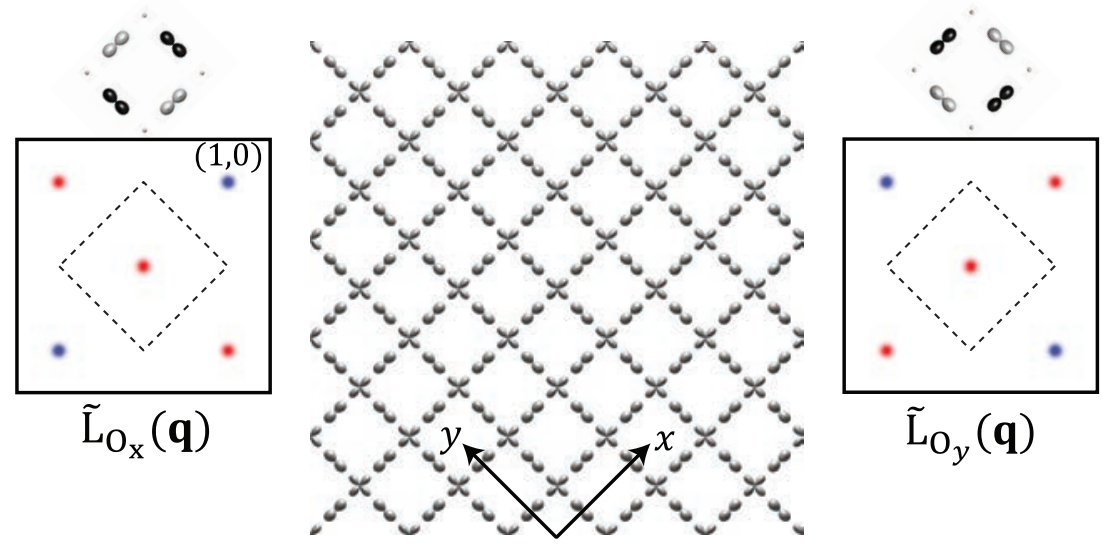

(b)
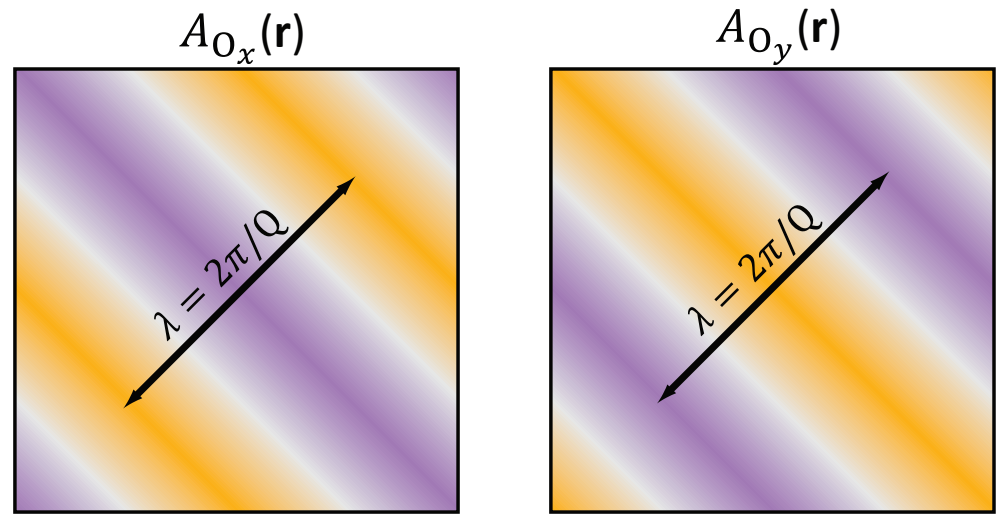

(c)

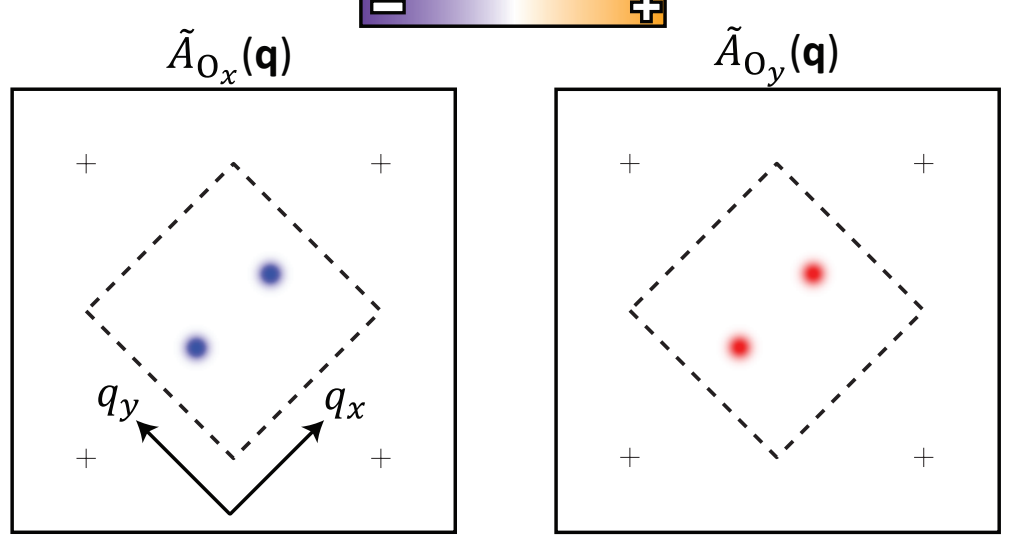

њ

Figure C.1: (a) Fourier transforms of the x-directed and y-directed oxygen sublattices without a CDW modulation. Gray orbitals signify those in the sub-lattice under consideration and black those in the other sub-lattice. (b) Schematic of continuous functions $A_{O_{x, y}}(\vec{r})$ that when multiplied by the sub-lattice functions $L_{O_{x, y}}(\vec{r})$ yield density waves in anti-phase on the two sub-lattices with a modulation along the $\mathrm{x}$ direction. (c) Fourier transforms of the functions $A_{O_{x, y}}(\vec{r})$ exhibiting a relative phase of $\pi$ as required for a $d$-symmetry form factor density wave. 
Thus, is follows immediately that

$$
\begin{gathered}
\tilde{O}_{x}^{0,0}=\tilde{A}(\vec{q}), \tilde{O}_{y}^{0,0}=-\tilde{A}(\vec{q}) \\
\tilde{O}_{x}^{1,0}=-\tilde{A}(\vec{q}), \tilde{O}_{y}^{1,0}=-\tilde{A}(\vec{q}) \\
\tilde{O}_{x}^{0,1}=\tilde{A}(\vec{q}), \tilde{O}_{y}^{0,1}=\tilde{A}(\vec{q})
\end{gathered}
$$

and hence

$$
\begin{array}{r}
\tilde{O}_{x}^{0,0}+\tilde{O}_{y}^{0,0}=0, \tilde{O}_{x}^{0,0}-\tilde{O}_{y}^{0,0}=2 \tilde{A}(\vec{q}) \\
\tilde{O}_{x}^{1,0}+\tilde{O}_{y}^{1,0}=-2 \tilde{A}(\vec{q}), \tilde{O}_{x}^{1,0}-\tilde{O}_{y}^{1,0}=0 \\
\tilde{O}_{x}^{0,1}+\tilde{O}_{y}^{0,1}=2 \tilde{A}(\vec{q}), \tilde{O}_{x}^{0,1}+\tilde{O}_{y}^{0,1}=0
\end{array}
$$

A direct consequence of a $d$-symmetry form factor is that in $\tilde{O}_{x}(\vec{q})+\tilde{O}_{y}(\vec{q})$ the amplitude of the convolution image at $(0,0)$ is canceled exactly whereas those at the $( \pm 1,0)$ and $(0, \pm 1)$ points are enhanced as illustrated in figures C.2 (b) and (c). The converse is true for $\tilde{O}_{x}(\vec{q})+\tilde{O}_{y}(\vec{q})$. This holds for any $d$-symmetry form factor modulation in the presence of arbitrary amplitude and overall phase disorder.

Empirically (as detailed in chapter 3), our data contain modulations at two wave vectors $\vec{Q}_{x}=(Q, 0)$ and $\vec{Q}_{y}=(0, Q)$ with $Q \approx 1 / 4$ but with a great deal of fluctuation in the spatial phase of the CDW [89]. Any $d$-symmetry form factor CDW containing two wave vectors $\vec{Q}_{x}$ and $\vec{Q}_{y}$ can be described by

$$
\begin{gathered}
A(\vec{r})=\operatorname{Re}\left[e^{i \vec{Q}_{x} \cdot \vec{r}} \cdot H_{x}(\vec{r})\right]+\operatorname{Re}\left[e^{i \vec{Q}_{y} \cdot \vec{r}} \cdot H_{y}(\vec{r})\right] \\
\tilde{A}(\vec{q})=\frac{1}{2}\left[\tilde{H}_{x}\left(\vec{q}-\vec{Q}_{x}\right)+\tilde{H}_{x}^{*}\left(\vec{q}+\vec{Q}_{x}\right)+\tilde{H}_{y}\left(\vec{q}-\vec{Q}_{y}\right)+\tilde{H}_{y}^{*}\left(\vec{q}+\vec{Q}_{y}\right)\right]
\end{gathered}
$$

The complex valued functions $H_{x, y}(\vec{r})$ locally modulate the amplitude and phase of the density wave and hence encode its disorder. The problem now reduces to performing the convolutions contained in equations C.4 and C.5.

For the specific example of $\vec{Q}_{x} \approx(1 / 4$,$) and \vec{Q}_{y} \approx(0,1 / 4)$ considered in our study, the predominantly $d$-symmetry form factor requires that the peaks at $\pm \vec{Q}_{x}$ and $\pm \vec{Q}_{y}$ present in both $\tilde{O}_{x}(\vec{q})$ and $\tilde{O}_{y}(\vec{q})$ must cancel exactly in $\tilde{O}_{x}(\vec{q})+\tilde{O}_{y}(\vec{q})$ and be enhanced in $\tilde{O}_{x}(\vec{q})-\tilde{O}_{y}(\vec{q})$. Conversely the peaks at $\vec{Q}^{\prime}=(1,0) \pm \vec{Q}_{x, y}$ and $\vec{Q}^{\prime \prime}=(0,1) \pm \vec{Q}_{x, y}$ will be enhanced in $\tilde{O}_{x}(\vec{q})+\tilde{O}_{y}(\vec{q})$ but will cancel exactly in $\tilde{O}_{x}(\vec{q})-\tilde{O}_{y}(\vec{q})$. These are 
(a)
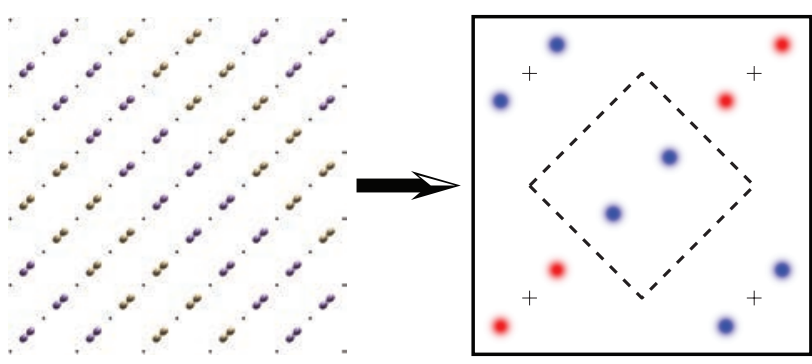

$\tilde{O}_{x}(\mathbf{q})$

$\tilde{A}_{\mathrm{O}_{x}}(\mathbf{q}) * \tilde{\mathrm{L}}_{\mathrm{O}_{\mathrm{x}}}(\mathbf{q})$

口 占
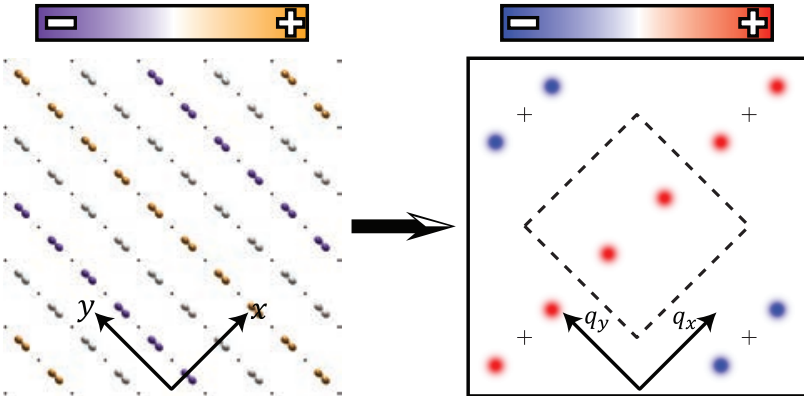

$\tilde{O}_{y}(\mathbf{q})$

$\tilde{A}_{\mathrm{O}_{y}}(\mathbf{q}) * \tilde{\mathrm{L}}_{\mathrm{O}_{\mathbf{y}}}(\mathbf{q})$

(b)
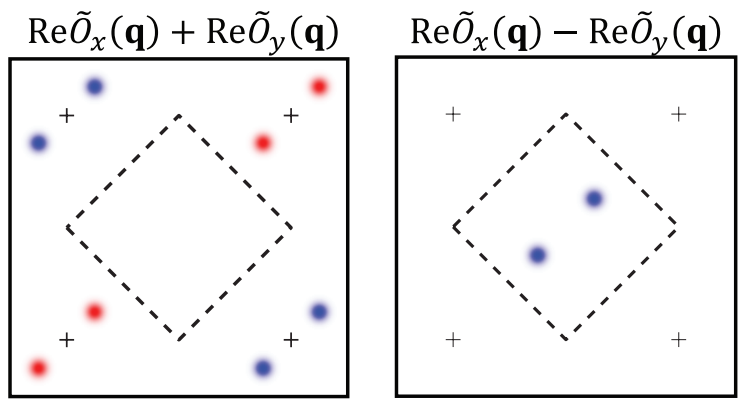

(c)
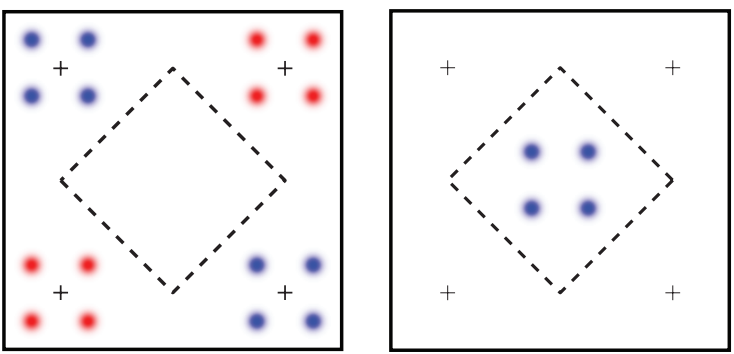

Figure C.2: (a) Schematic of the segregated sub-lattice images $O_{x, y}(\vec{r})$ and their Fourier transforms $\tilde{O}_{x, y}(\vec{q})$ that can be obtained from figure C.1 by application of the convolution theorem. (b) Sum and difference of $\operatorname{Re} \tilde{O}_{x}$ and $\operatorname{Re} \tilde{O}_{y}$ for a $d$-symmetry form factor CDW with modulation along the x direction at $\vec{Q}_{x}=(Q, 0)$. Note that the origin of coordinates in real space has been chosen such that the Fourier transforms are purely real. (c) Sum and difference of $\operatorname{Re} \tilde{O}_{x}$ and $\operatorname{Re} \tilde{O}_{y}$ for a $d$-symmetry form factor CDW with modulations along the $\mathrm{x}$ and y directions at $\vec{Q}=(Q, 0),(0, Q)$. The key signature of the d-symmetry form factor is the absence of the peaks at $(Q, 0),(0, Q)$ in $\operatorname{Re} \tilde{O}_{x}(\vec{q})+\operatorname{Re} \tilde{O}_{y}(\vec{q})$ and their presence in $\operatorname{Re} \tilde{O}_{x}(\vec{q})-\operatorname{Re} \tilde{O}_{y}(\vec{q})$; the converse is true for the CDW peaks surrounding $( \pm 1,0)$ and $(0, \pm 1)$. 
necessary consequences of a CDW with a $d$-symmetry form factor. This is discussed in the chapter 4 and in accord with the observations in figures 4.7 . 


\section{Appendix D}

\section{Sub-Lattice Phase-Resolved}

\section{Fourier Transforms}

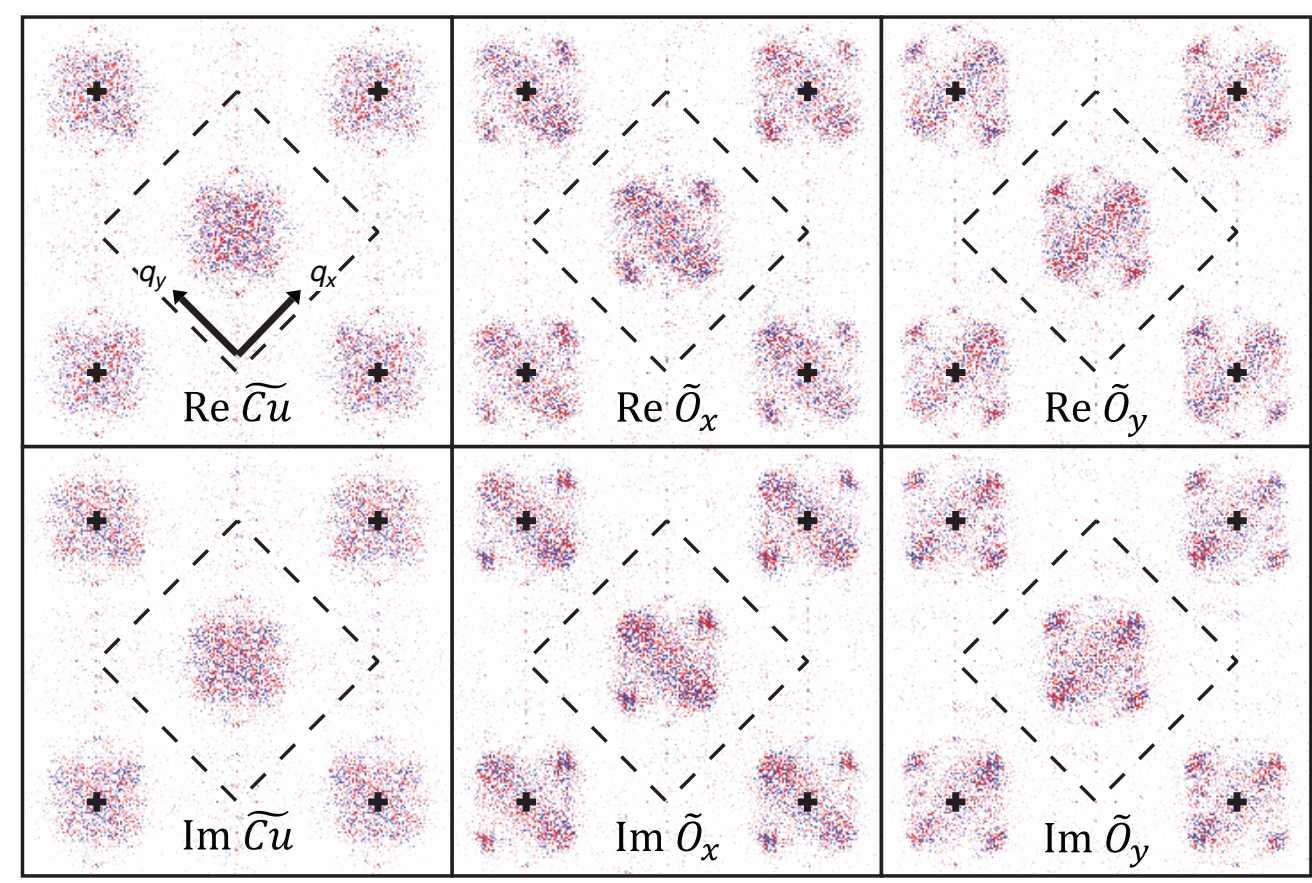

口 फ

Figure D.1: Real and imaginary parts of the sub-lattice segregated Fourier transforms $\tilde{C} u(\vec{q}), \tilde{O}_{x}(\vec{q})$ and $\tilde{O}_{y}(\vec{q})$ derived from $R(\vec{r},|E|=150 \mathrm{meV}$ ) (figure 4.6) for a $\mathrm{Bi}_{2} \mathrm{Sr}_{2} \mathrm{CaCu}_{2} \mathrm{O}_{8+\delta}$ sample with hole concentration $p=8 \pm 1 \%$. 


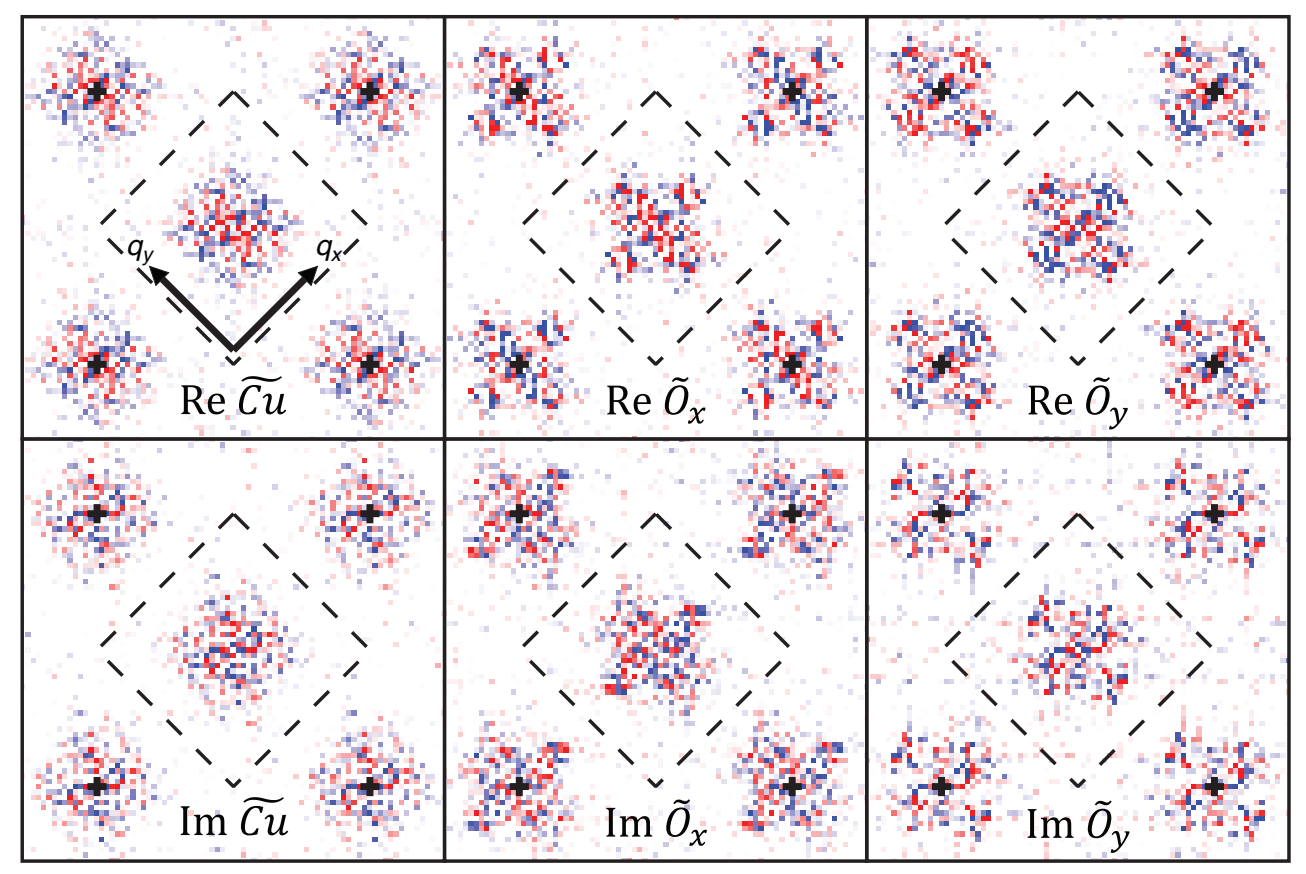

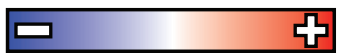

Figure D.2: Real and imaginary parts of the sub-lattice segregated Fourier transforms $\tilde{C} u(\vec{q}), \tilde{O}_{x}(\vec{q})$ and $\tilde{O}_{y}(\vec{q})$ derived from $Z(\vec{r},|E|=150 \mathrm{meV}$ ) (figure 3.1 ) for a $\mathrm{Na}_{x} \mathrm{Ca}_{2-x} \mathrm{CuO}_{2} \mathrm{Cl}_{2}$ sample with hole concentration $p=12 \pm 1 \%$. 


\section{Appendix E}

\section{Topographic Imaging of the Cuprate $d$-Symmetry Form}

\section{Factor Charge Density Wave}

In chapter 4 we studied the $d$-symmetry form factor charge density wave (CDW) in under-doped cuprates using spectroscopic-imaging STM (SI-STM) by analysing the spectroscopic maps $g(\vec{r}, E), I(\vec{r}, E)$ defined in chapter 2, and their derivatives $Z(\vec{r},|E|)=$ $g(\vec{r},+|E|) / g(\vec{r},-|E|)$ and $R(\vec{r},|E|)=I(\vec{r},+|E|) / I(\vec{r},-|E|)$. From these we then determined that the CDW in under-doped $\mathrm{Bi}_{2} \mathrm{Sr}_{2} \mathrm{CaCu}_{2} \mathrm{O}_{8+\delta}$ and $\mathrm{Na}_{x} \mathrm{Ca}_{2-x} \mathrm{CuO}_{2} \mathrm{Cl}_{2}$ has a predominantly $d$-symmetry form factor by examining the sub-lattice segregated Fourier transform $\tilde{D}(\vec{q})=\tilde{O}_{x}(\vec{q})-\tilde{O}_{y}(\vec{q})$ as detailed in chapters 3 and 4 .

At dopings near optimal, it is known from several experimental techniques that the intensity of the $d$-symmetry form factor density wave diminishes rapidly in $\mathrm{Bi}_{2} \mathrm{Sr}_{2} \mathrm{CaCu}_{2} \mathrm{O}_{8+\delta}$ [29]. Therefore, to improve the visualisation of the $d$-symmetry form factor density wave signature at such high-dopings as used here, we use topographic information instead. In constant-current topographic imaging, the STM feedback system adjusts the tip-sample separation, $z$, as it scans over the sample surface to maintain a set-point current, $I_{s}$, at a constant applied tip-sample bias $V_{s}$. The topographic image $T(\vec{r})$ is related to the superconductor-insulator-normal metal (SIN) tunnelling current

$$
I(\vec{r}, z, E=e V)=f(\vec{r}, z) \int_{0}^{e V_{s}} n_{s}(\vec{r}, \epsilon) d \epsilon
$$


where $n_{s}(\vec{r}, \epsilon)$ is the sample density of states. Here $f(\vec{r}, z)$ encodes all matrix-element variations, which includes those due to surface corrugation, work-function variation and the form of sample and tip wave-functions.

Motivated by standard results from the WKB approximation in the strongly attenuating barrier limit and the work of Tersoff and Hamman described in chapter 2 we assume that $f(\vec{r}, z)$ takes the form

$$
f(\vec{r}, \vec{z})=\exp (-\kappa z) A(\vec{r})
$$

where $\kappa$ is a tunnelling decay factor that depends on the work functions of the sample and tip. In constant-current topographic mode the value of $z$ recorded in the topographic image $T(\vec{r})$ is then

$$
T(\vec{r})=\frac{1}{\kappa} \ln \left[\int_{0}^{e V_{s}} n_{s}(\vec{r}, \epsilon) d \epsilon\right]+\frac{1}{\kappa} \ln \left[\frac{I_{s}}{A(\vec{r})}\right] .
$$

Thus high signal-to-noise topographic images obtained by constant-current STM imaging reveal contributions from both the surface structure and variations in $n_{s}(\vec{r}, E)$.

While a single cuprate topographic image will contain the signature of the $d$-symmetry form factor CDW, subtracting one topographic image from another taken at opposite bias polarity can further enhance the cuprate CDW signal. This is because, as demonstrated in chapter 4 , the $d$-symmetry form factor CDW modulations at the pseudo-gap energy scale, $|E| \sim \Delta_{1}$ in $\mathrm{Bi}_{2} \mathrm{Sr}_{2} \mathrm{CaCu}_{2} \mathrm{O}_{8+\delta}$ and $\mathrm{Na}_{x} \mathrm{Ca}_{2-x} \mathrm{CuO}_{2} \mathrm{Cl}_{2}$ modulates in antiphase between empty and filled states. Hence, a subtraction of topographic images $T^{+}(\vec{r})$ and $T^{-}(\vec{r})$, formed with biases $+V_{s}$ and $-V_{s}$ and with $e V_{s} \gtrsim \Delta_{1}$ should amplify the CDW contrast. Indeed using equation E.3 one can see that this should be related to the spectroscopic map $R(\vec{r},|E|)$ via

$$
T^{+}(\vec{r})-T^{-}(\vec{r})=\frac{1}{\kappa} \ln \left[\frac{\int_{0}^{e V_{s}} n_{s}(\vec{r}, \epsilon) d \epsilon}{\int_{0}^{-e V_{s}} n_{s}(\vec{r}, \epsilon) d \epsilon}\right]=\frac{1}{\kappa} \ln \left[R\left(\vec{r},\left|e V_{s}\right|\right)\right],
$$

under the assumption that the factor $A(\vec{r})$ is the same for positive and negative biases.

In figures E.1 (a) and (b) I demonstrate this procedure using two high-resolution, highsignal-to-noise ratio topographs $T(\vec{r})$ taken at opposite bias polarities $(80 \mathrm{mV}$ and $80 \mathrm{mV}$ ) with the same conventional tungsten tip for a $\mathrm{Bi}_{2} \mathrm{Sr}_{2} \mathrm{CaCu}_{2} \mathrm{O}_{8+\delta}$ sample with 
$p=0.17$. They are spatially registered to each other with picometre precision using the algorithm given in section 3.5. Figure E.1 (c) is the difference of these topographs, from which the two oxygen sub-lattice segregated images $O_{x}(\vec{r}) ; O_{y}(\vec{r})$ are then extracted and $\tilde{D}(\vec{q})=\tilde{O}_{x}(\vec{q})-\tilde{O}_{y}(\vec{q})$ calculated. $\tilde{D}(\vec{q})$, shown here in figure E.1 (d) and figure 7.2 (d) in chapter 7 , reveals a predominantly $d$-symmetry form factor CDW because of the existence of four maxima at the CDW wave-vector $Q_{C}=( \pm 0.22,0) 2 \pi / a_{0}$ and $(0, \pm 0.22) 2 \pi / a_{0}$.
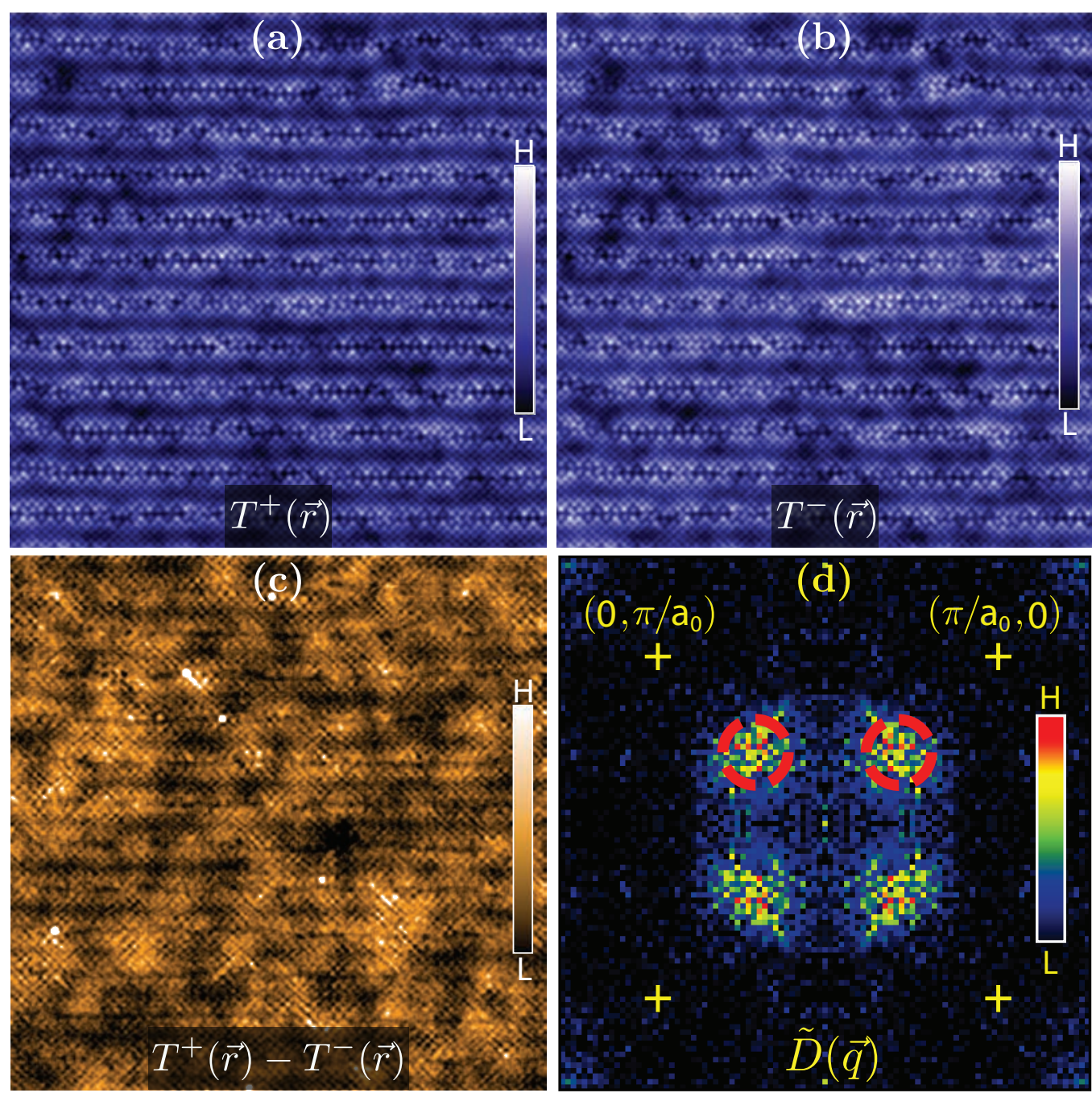

FiguRE E.1: (a) Intra unit-cell resolution topograph of $\mathrm{Bi}_{2} \mathrm{Sr}_{2} \mathrm{CaCu}_{2} \mathrm{O}_{8+\delta}$ sample with $p=0.17$, taken with $V_{s}=+80 \mathrm{mV}$ and $I_{s}=30 \mathrm{pA}$. (b) Topograph in identical field of view as (a) and registered to (a) with picometer precision. An setup bias polarity opposite to that in (a) was used with $V_{s}=-80 \mathrm{mV}$ and $I_{s}=30 \mathrm{pA}$. (c) Difference between (a) and (b) showing clear $d$-symmetry form factor CDW modulations. Their contrast is increased in this subtraction due to a $\pi$ phase shift between the modulations present in (a) and (b). (d) Fourier transform $\tilde{D}(\vec{q})=\tilde{O}_{x}(\vec{q})-\tilde{O}_{y}(\vec{q})$ calculated from (c) revealing $d$-symmetry form factor CDW modulations at wave-vector $Q_{C}=( \pm 0.22,0) 2 \pi / a_{0}$ and $(0, \pm 0.22) 2 \pi / a_{0}$. This Fourier transform is symmetrised about the vertical axis. 



\section{Appendix F}

\section{Absence of Setup Effect in Relative Spatial Phase}

\section{Measurements}

In section 4.6 I reported a spatial $\pi$ phase shift between CDW modulations at wavevectors $\vec{Q} \approx(0,0.25)(0.25,0) 2 \pi / a_{0}$ in $g\left(\vec{r}, E=+\Delta_{1}\right)$ and $g\left(\vec{r}, E=-\Delta_{1}\right)$ for a $\mathrm{Bi}_{2} \mathrm{Sr}_{2} \mathrm{CaCu}_{2} \mathrm{O}_{8+\delta}$ sample with $p \approx 6 \%$. In this appendix I demonstrate that this is not due to the setup effect artifact discussed in section 2.4.1.

As detailed in chapter 2 the differential tunnelling conductance is given by

$$
g(\vec{r}, E=e V)=I_{S} \frac{n(\vec{r}, e V)}{\int_{0}^{e V_{S}} n(\vec{r}, \epsilon) d \epsilon},
$$

where $I_{S}$ and $V_{S}$ are the set-point current and voltage respectively. The term in the denominator is responsible for the setup effect since it imprints the integral of the local density of states (LDOS) over the range of energies 0 to $e V_{s}$ onto every energy in $g(\vec{r}, E=$ $e V)$.

For example, if $\int_{0}^{e V_{S}} n(\vec{r}, \epsilon) d \epsilon$ contains modulations at wave-vector $\vec{Q}$, and $n\left(\vec{r}, E=E_{1}\right)$ does not contain any modulations at this wave-vector, $g\left(\vec{r}, E_{1}\right)$ will exhibit modulations at wave-vector $\vec{Q}$ but in spatial anti-phase with $\int_{0}^{e V_{S}} n(\vec{r}, \epsilon) d \epsilon$. Clearly this modulation in $g\left(\vec{r}, E_{1}\right)$ does not reflect any physical modulation in the local density of states at energy $E_{1}$. 
To guard against this effect in the collection of the data presented in section 4.6, we found set-point parameters $V_{S}$ and $I_{S}$ for which $\int_{0}^{e V_{S}} n(\vec{r}, \epsilon) d \epsilon$ does not exhibit any $d$-symmetry form factor modulations at $\vec{Q} \approx(0,0.25)(0.25,0) 2 \pi / a_{0}$ for a specific hole concentration of $p=6 \%$. To establish that we had indeed found $V_{S}$ and $I_{S}$ satisfying these conditions we used the simultaneous topograph to the spectroscopic data presented in figure $4.16(p=6 \%)$.

As detailed in appendix E, the topographic image $T(\vec{r})$ can be expressed as

$$
T(\vec{r})=\frac{1}{\kappa} \ln \left[\int_{0}^{e V_{s}} n_{s}(\vec{r}, \epsilon) d \epsilon\right]+\frac{1}{\kappa} \ln \left[\frac{I_{s}}{A(\vec{r})}\right],
$$

where $\kappa$ is a tunnelling decay factor that depends on the work functions of the sample and tip. $A(\vec{r})$ encodes all other spatial dependencies of the tunnelling matrix elements. The first term in equation F.2 measures exactly the denominator of equation F.1 that gives rise to the setup effect, i.e the integrated density of states. Thus, any modulations in $\int_{0}^{e V_{s}} n_{s}(\vec{r}, \epsilon) d \epsilon$ should be apparent in $T(\vec{r})$.

In figure F.1 (a) I show the topographic image, $T(\vec{r})$, simultaneous to the spectroscopic data shown in figure 4.16. In figure F.1 (b) I show the Fourier transform $\left|\tilde{D}^{T}(\vec{q})\right|$ derived from the topograph in (a). No modulations are detected at wavevectors $\vec{Q} \approx(0,0.25)(0.25,0) 2 \pi / a_{0}$, signifying that there are no $d$-symmetry form factor modulations in $\int_{0}^{e V_{S}} n(\vec{r}, \epsilon) d \epsilon$ at these wave-vectors. Thus, the spatial $\pi$ phase shift between $d$-symmetry form factor CDW modulations in $g\left(\vec{r}, E=+\Delta_{1}\right)$ and $g\left(\vec{r}, E=-\Delta_{1}\right)$ reported in section 4.6 is not due to the setup effect. 
(a)

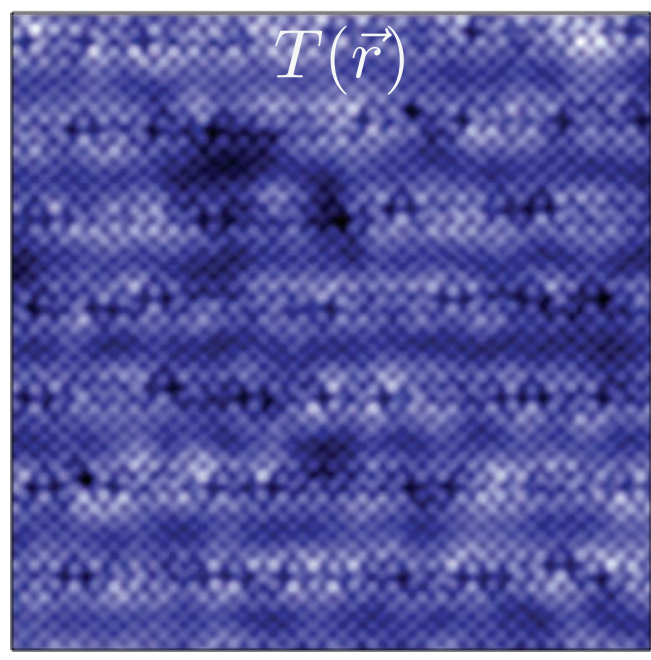

(b)

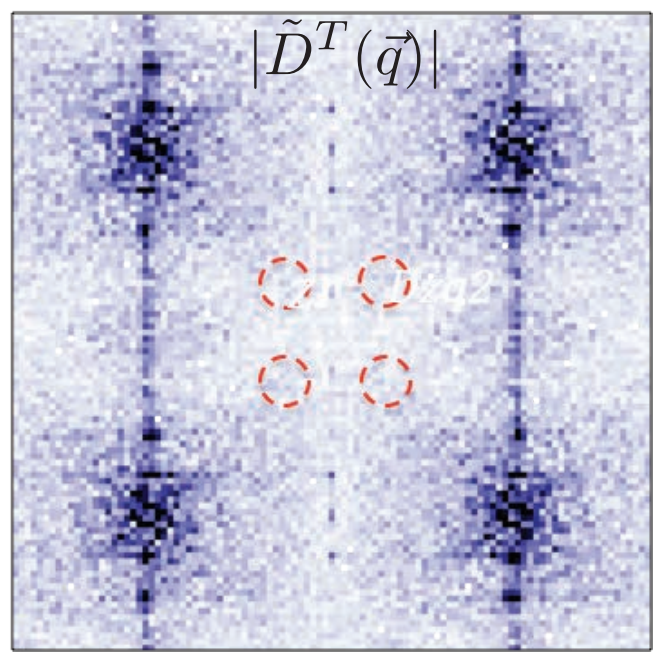

Figure F.1: (a) Simultaneous topograph, $T(\vec{r})$ to spectroscopic data shown in figure 4.16 for a $\mathrm{Bi}_{2} \mathrm{Sr}_{2} \mathrm{CaCu}_{2} \mathrm{O}_{8+x}$ sample with $p \sim 6 \%$. A set-point voltage $V_{S}=250 \mathrm{mV}$ was used to acquire the topograph. (b) Fourier transform $\left|\tilde{D}^{T}(\vec{q})\right|$ derived from the topograph in (a). There are no peaks at the wave-vectors $\vec{Q} \approx(0,0.25)(0.25,0) 2 \pi / a_{0}$ (red circles). 

Appendix G

Antiphase Modulation of Filled and Empty States at $|E|=\Delta_{1}$ 

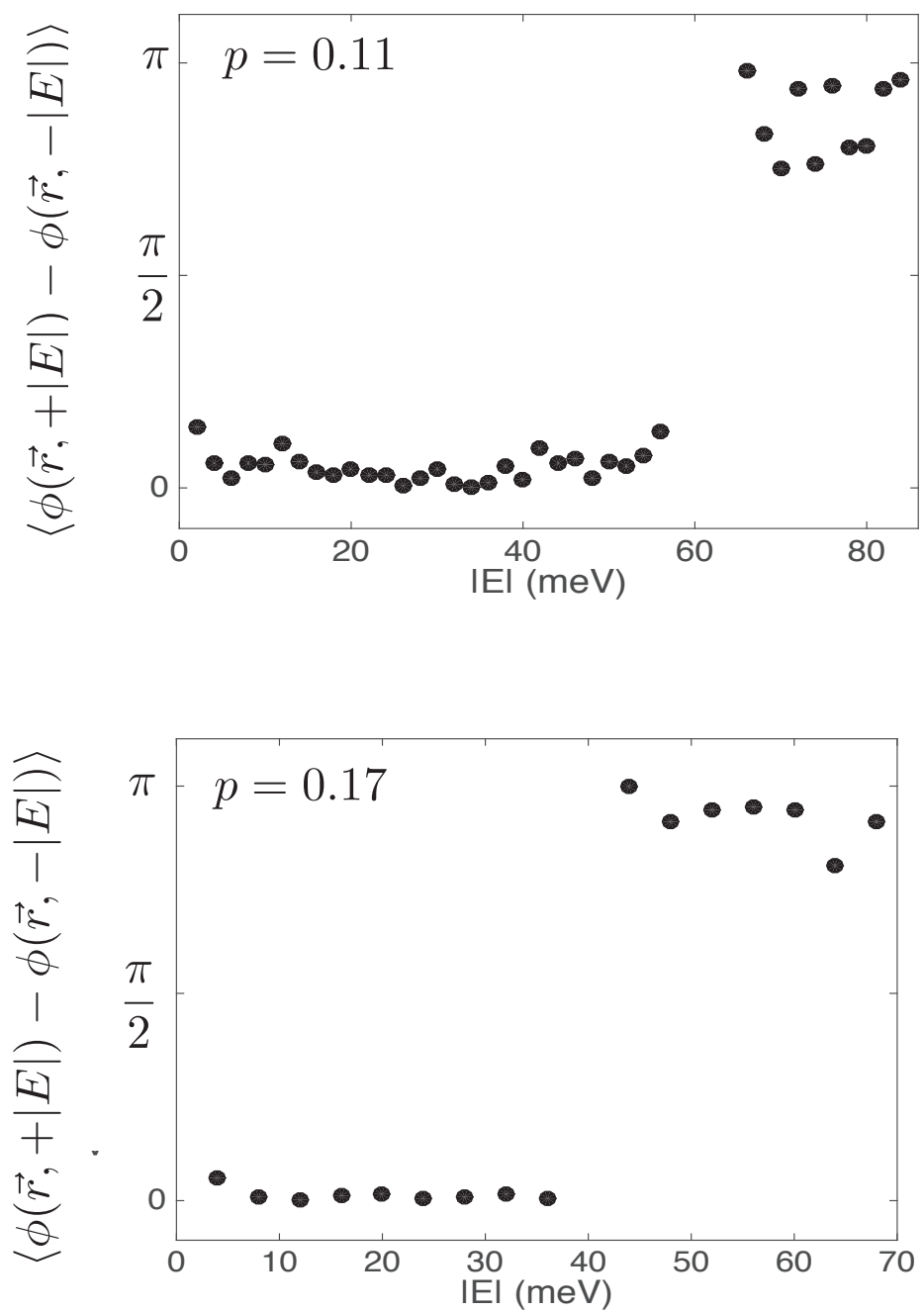

Figure G.1: Field of view average of difference in spatial phase of $d$-symmetry form factor CDW modulations in $g(\vec{r}, E)$ above and below the chemical potential. Data is shown for $\mathrm{Bi}_{2} \mathrm{Sr}_{2} \mathrm{CaCu}_{2} \mathrm{O}_{8+\delta}$ samples with $p=0.11$ and $p=0.17$. As in figure 4.16, CDW modulations at $|E| \sim \Delta_{1}$ acquire a $\pi$ phase shift between empty and filled states. In conjunction with the results presented in figure 4.16 this demonstrates that this phenomenology is consistent across a range of doings $0.06 \leq p \leq 0.17$, spanning the pseudogap regime. 


\section{Appendix $\mathbf{H}$}

\section{Measuring $R_{N}$ for Cuprate SJTM}

In principle, SJTM allows direct measurement of modulations in the superconducting order parameter amplitude through the product $I_{J} R_{N}$. Here $I_{J}$ is the intrinsic Josephson critical current of the junction and $R_{N}$ is a characteristic resistance of the single-particle tunnelling channel. In taking this product, variations that affect the matrix elements for tunnelling of Cooper pairs and the single-particle tunnelling conductance $\left(1 / R_{N}\right)$ in the same way cancel out.

We use the differential conductance $d I / d V_{B}$ at a bias voltage $V_{B}$ far higher than the SIS gap edge as an estimate of the ungapped, "normal state", junction conductance $G_{N}$. Simultaneous measurement of this value and $I_{c}(\vec{r})$ were not possible because the former requires $V_{B}$ in the hundreds of millivolts while the latter requires $V_{B}$ of a few tens of microvolts. Moreover, in the picometer range tip-sample separations used in measuring $I_{c}(\vec{r})$, the single-particle tunnelling current can become destructively large to our $\mathrm{Bi}_{2} \mathrm{Sr}_{2} \mathrm{CaCu}_{2} \mathrm{O}_{8+\delta}$ nanoflake tip. Thus the following procedure was adopted:

1. Measure $I_{c}(\vec{r})$ with set-point current $I_{s}=I_{s 1}$ and bias voltage $V_{s}=V_{s 1}$, as shown in figure H.1 (a).

2. In the same field of view measure $g_{1}(\vec{r}, E)$, defined as $d I / d V$ for bias voltages $V_{s 1}$ to $-V_{s 1}$ with set-point condition $I_{s}=I_{s 1}, V_{s}=V_{s 1}$.

3. In the same field of view measure $g_{2}(\vec{r}, E)$ defined as $d I / d V$ with set-point condition $I_{s}=I_{s 2}, V_{s}=V_{s 2}$ for bias voltages $V_{s 2}$ to $-V_{s 2}$, where $V_{s 2} \gg V_{s 1}$ and $V_{s 2}>\Delta_{S I S}$. 
4. Over the range $V=\left[-V_{s 1}, V_{s 1}\right]$ determine the coefficients $\alpha(\vec{r})$ and $\beta(\vec{r})$ which scale $g_{2}(\vec{r}, E)$ onto $g_{1}(\vec{r}, E)$ via $g_{1}(\vec{r}, E)=\alpha(\vec{r}) g_{2}(\vec{r}, E)+\beta(\vec{r})$. In doing the scaling a small region of voltages near $V=0$ where the Cooper-pair tunnelling (Josephson) signal exhibits is excluded.

5. $R_{N}(\vec{r})$ for junctions used to measure $I_{c}(\vec{r})$ is then given by $1 / R_{N}(\vec{r})=G_{N}(\vec{r})=$ $\frac{\alpha(\vec{r})}{2}\left[g_{2}\left(\vec{r},+V_{s 2}\right)+g_{2}\left(\vec{r},-V_{s 2}\right)\right]+\beta(\vec{r})$ as shown in figure H.1 (b).

Now we consider the case of the cuprate supermodulation PDW. The bulk quasi-periodic distortion of the $\mathrm{Bi}_{2} \mathrm{Sr}_{2} \mathrm{CaCu}_{2} \mathrm{O}_{8+\delta}$ crystal at wave-vector $\vec{Q}_{S M}$ leads to modulations of the measured $I_{c}(\vec{r})$ at the same wave-vector superposed on the larger background,

$$
\begin{aligned}
|\Psi(\vec{r})| \propto I_{J}(\vec{r}) R_{N}(\vec{r}) & =\left(I_{J}^{0}+I_{J}^{P} \cos \left(\vec{Q}_{S M} \cdot \vec{r}\right)\right)\left(R_{N}^{0}+R_{N}^{P} \cos \left(\vec{Q}_{S M} \cdot \vec{r}+\phi\right)\right) \\
& =I_{J}^{0} R_{N}^{0}+R_{N}^{0} I_{J}^{P} \cos \left(\vec{Q}_{S M} \cdot \vec{r}\right)+I_{J}^{0} R_{N}^{P} \cos \left(\vec{Q}_{S M} \cdot \vec{r}+\phi\right)+\ldots
\end{aligned}
$$

where I allow for an arbitrary phase difference $\phi$ between modulations in $I_{J}$ and $R_{N}$ at $\vec{Q}_{S M}$. For any value of $\phi$, one may conclude that the modulation in $I_{J}$ at $\vec{Q}_{S M}$ is, at least in part, due to a modulation in $\Psi(\vec{r})$ if

$$
\frac{R_{N}^{P}}{R_{N}^{0}} / \frac{I_{J}^{P}}{I_{J}^{0}}<1 .
$$

In the experiments presented in chapters 5 and 7 we measure the quantity

$$
I_{c}(\vec{r}) \propto\left(I_{J}^{0}\right)^{2}+2 I_{J}^{0} I_{J}^{P} \cos \left(\vec{Q}_{S M} \cdot \vec{r}\right)+\ldots
$$

as shown in figure H.1 (a). In figure H.1 (b) I show $R_{N}(\vec{r})$ derived as above. By comparing the amplitude of $\tilde{I}_{c}(\vec{q})$ and $\tilde{R}_{N}(\vec{q})$ at $\vec{q}=\vec{Q}_{S M}$, normalised to their $\vec{q}=0$ value, one may determine $\frac{R_{N}^{P}}{R_{N}^{0}} / \frac{I_{J}^{P}}{I_{J}^{0}}$ as in figure H.1 (c). We find that this ratio has a value of $\approx 0.25$ and thus modulations in $I_{c}(\vec{r})$ at $\vec{Q}_{S M}$ can be regarded as being due to those in $\Psi(\vec{r})$.

The inset to figure H.1 (c) is $\left|\tilde{R}_{N}(\vec{q})\right|$, showing directly that variations in $R_{N}(\vec{r})$ near $\vec{q} \approx( \pm 0.25,0) ;(0, \pm 0.25) 2 \pi / a_{0}$ are negligible, so that modulations in $I_{c}(\vec{r})$ at those wave-vectors are those of the condensate itself. 

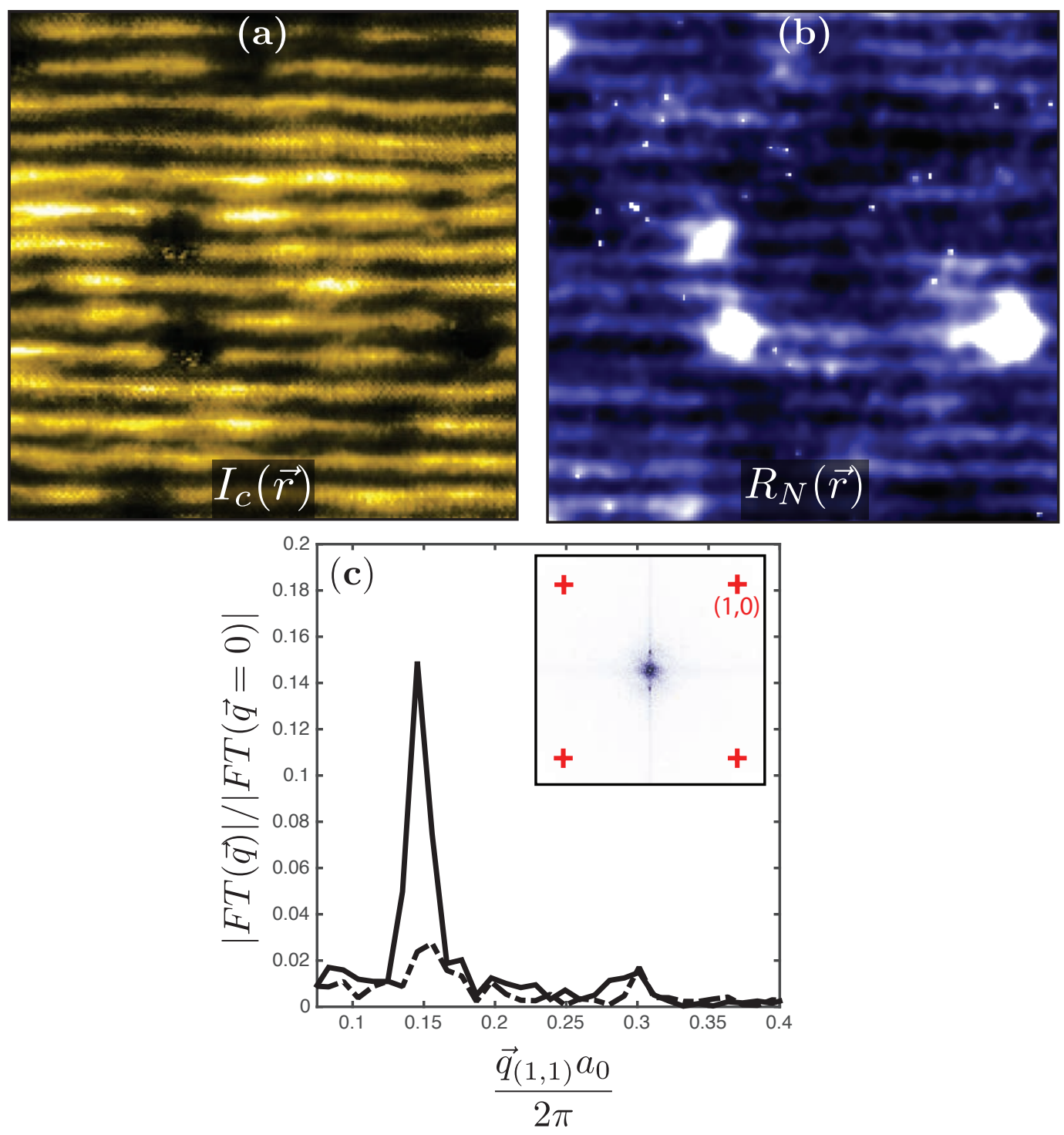

Figure H.1: (a) A typical measured $I_{c}(\vec{r})$ image of $\mathrm{Bi}_{2} \mathrm{Sr}_{2} \mathrm{CaCu}_{2} \mathrm{O}_{8+\delta}$ with the crystal super-modulation effect retained and apparent as strong spatial modulations in $I_{c}$ along the vertical axis. (b) Measured $R_{N}(\vec{r})$ image in the same field of view as (a), with the crystal super-modulation effect retained. The spatial modulations in $R_{N}(\vec{r})$ along the vertical axis are greatly diminished in relative amplitude compared to $I_{c}(\vec{r})$ modulations in (a). (c) The inset shows $\left|\tilde{R}_{N}(\vec{q})\right|$, the magnitude of the Fourier transform of $R_{N}(\vec{r})$ from (b). It shows that variations in $R_{N}(\vec{r})$ near $\vec{q} \approx( \pm 0.25,0) ;(0, \pm 0.25) 2 \pi / a_{0}$ are negligible. Plotting the simultaneously measured Fourier amplitudes of $\tilde{I}_{c}(\vec{q})$ (solid line) and $\widetilde{R}_{N}(\vec{q})$ (dashed line) along the $(1,1)$ direction passing through the wave-vector of the super-modulation $\vec{Q}_{S M}$ shows that $\frac{R_{N}^{P}}{R_{N}^{0}} / \frac{I_{J}^{P}}{I_{J}^{0}} \approx 0.25<1$. This, together with the inset, indicates that modulations in $I_{c}(\vec{r})$ at $\vec{Q}_{S M}$ and near $\vec{q} \approx( \pm 0.25,0) ;(0, \pm 0.25) 2 \pi / a_{0}$ are primarily arising from the variations of the superconducting condensate. 



\section{Bibliography}

[1] J. Bednorz and K. Muller. "Possible High $\mathrm{T}_{c}$ Superconductivity in the Ba - La Cu - O System". Zeitschrift für Physik B: Condensed Matter 64 (1986), pp. 189193.

[2] J. G. Bednorz and K. A. Müller. "Perovskite-Type Oxides - The New Approach to High-T ${ }_{c}$ Superconductivity". Nobel Lectures, Physics 1981-1990 (1993), pp. 424457.

[3] A. P. Drozdov et al. "Conventional superconductivity at 203 kelvin at high pressures in the sulfur hydride system". Nature 525.7567 (2015), pp. 73-76.

[4] P. W. Anderson. Basic Notions in Condensed Matter Physics. Menlo Park, CA: The Benjamin/Cummings Publishing Company, 1984.

[5] L. D. Landau. Collected papers of L. D. Landau. Gordon and Breach, 1965.

[6] W. Meissner and R. Ochsenfeld. "Ein neuer Effekt bei Eintritt der Supraleitfhigkeit". Die Naturwissenschaften 21.44 (1933), pp. 787-788.

[7] D. V. Delft and P. Kes. "The discovery of superconductivity". Physics Today 63.9 (2010), pp. 38-42.

[8] J. Bardeen, L. N. Cooper, and J. R. Schrieffer. "Theory of Superconductivity". Physical Review 108.5 (1957), pp. 1175-1204.

[9] T. Giamarchi. Quantum Physics in One Dimension. Vol. 6. 38. Oxford University Press, 2003, pp. 1-28.

[10] N. N. Bogoliubov. "On a new method in the theory of superconductivity". Il Nuovo Cimento 7.6 (1958), pp. 794-805.

[11] C. Tsuei and J. Kirtley. "Pairing symmetry in cuprate superconductors". Reviews of Modern Physics 72.4 (2000), pp. 969-1016. 
[12] M. Tinkham. "Tunneling generation, relaxation, and tunneling detection of holeelectron imbalance in superconductors". Physical Review B 6.5 (1972), pp. 17471756.

[13] M. Ogata and H. Fukuyama. "The $\mathrm{t}-\mathrm{J}$ model for the oxide high- T c superconductors". Reports on Progress in Physics 71.3 (2008), p. 36501.

[14] J. Zaanen, G. A. Sawatzky, and J. W. Allen. "Band gaps and electronic structure of transition-metal compounds". Physical Review Letters 55.4 (1985), pp. 418421.

[15] A. Fujimori et al. "Spectroscopic evidence for strongly correlated electronic states in La-Sr-Cu and Y-Ba-Cu oxides". Physical Review B 35.16 (1987), pp. 88148817.

[16] T. Takahashi et al. "Synchrotron-radiation photoemission study of the high- $\mathrm{T}_{c}$ Superconductor $\mathrm{YBa}_{2} \mathrm{Cu}_{3} \mathrm{O}_{7-\delta}$ ". Physical Review B 36.10 (1987), pp. 5686-5689.

[17] A. Bianconi et al. "Localization of $\mathrm{Cu} 3 \mathrm{~d}$ levels in the high $\mathrm{T}_{c}$ superconductor $\mathrm{YBa}_{2} \mathrm{Cu}_{3} \mathrm{O}_{7}$ by $\mathrm{Cu} 2 \mathrm{p}$ X-ray photoelectron spectroscopy". Solid State Communications 63.12 (1987), pp. 1135-1139.

[18] V. J. Emery. "Theory of high-Tc superconductivity in oxides". Physical Review Letters 58.26 (1987), pp. 2794-2797.

[19] F. C. Zhang and T. M. Rice. "Effective Hamiltonian for the superconducting $\mathrm{Cu}$ oxides". Physical Review B 37.7 (1988), pp. 3759-3761.

[20] P. Fazekas. Lecture Notes on Electron Correlation and Magnetism. 1999.

[21] L. Gao et al. "Superconductivity up to $164 \mathrm{~K}$ in $\mathrm{HgBa}_{2} \mathrm{Ca}_{m-1} \mathrm{Cu}_{m} \mathrm{O}_{2 m+2+\delta}$ ( $\mathrm{m}$ $=1,2$, and 3) under quasihydrostatic pressures". Physical Review B 50.6 (1994), pp. $4260-4263$.

[22] B. Keimer et al. "From quantum matter to high-temperature superconductivity in copper oxides." Nature 518.7538 (2015), pp. 179-86.

[23] J. N. Bruin et al. "Similarity of Scattering Rates in Metals Showing T-Linear Resistivity". Science 339.6121 (2013), pp. 804-807.

[24] A. Damascelli, Z. Hussain, and Z.-X. Shen. "Angle-resolved photoemission studies of the cuprate superconductors". Reviews of Modern Physics 75.2 (2003), pp. 473541. 
[25] A. A. Kordyuk. "Pseudogap from ARPES experiment: Three gaps in cuprates and topological superconductivity". Low Temperature Physics 41.5 (2015), pp. 319341.

[26] Ø. Fischer et al. "Scanning tunneling spectroscopy of high-temperature superconductors". Reviews of Modern Physics 79.1 (2007), pp. 353-419.

[27] K. Fujita et al. "Spectroscopic Imaging Scanning Tunneling Microscopy Studies of Electronic Structure in the Superconducting and Pseudogap Phases of Cuprate High-Tc Superconductors". Journal of the Physical Society of Japan 81.1 (2012), p. 011005.

[28] A. Yazdani, E. H. da Silva Neto, and P. Aynajian. "Spectroscopic Imaging of Strongly Correlated Electronic States". Annual Review of Condensed Matter Physics 7.1 (2016), pp. 11-33.

[29] K. Fujita et al. "Simultaneous Transitions in Cuprate Momentum-Space Topology and Electronic Symmetry Breaking”. Science 344.6184 (2014), pp. 612-616.

[30] Y. He et al. "Fermi surface and pseudogap evolution in a cuprate superconductor." Science 344.6184 (2014), pp. 608-11.

[31] I. M. Vishik et al. "A momentum-dependent perspective on quasiparticle interference in $\mathrm{Bi}_{2} \mathrm{Sr}_{2} \mathrm{CaCu}_{2} \mathrm{O}_{8+\delta}$ ". Nature Physics 5.10 (2009), pp. 718-721.

[32] M. J. Lawler et al. "Intra-unit-cell electronic nematicity of the high- $\mathrm{T}_{c}$ copperoxide pseudogap states." Nature 466.7304 (2010), pp. 347-51.

[33] Y. Li et al. "Hidden magnetic excitation in the pseudogap phase of a high-Tc superconductor". Nature 468.7321 (2010), pp. 283-285.

[34] P. Hosur et al. "Kerr effect as evidence of gyrotropic order in the cuprates". Physical Review B 87.11 (2013), pp. 1-8.

[35] J. Xia et al. "Polar kerr-effect measurements of the high-temperature $\mathrm{YBa}_{2} \mathrm{Cu}_{3} \mathrm{O}_{6+x}$ superconductor: Evidence for broken symmetry near the pseudogap temperature". Physical Review Letters 100.12 (2008), pp. 3-6.

[36] H. Karapetyan et al. "Magneto-optical measurements of a cascade of transitions in superconducting $\mathrm{La}_{1.875} \mathrm{Ba}_{0.125} \mathrm{CuO}_{4}$ Single Crystals". Physical Review Letters 109.14 (2012), pp. 1-5. 
[37] P. Hosur et al. "Erratum: Kerr effect as evidence of gyrotropic order in the cuprates [Phys. Rev. B 87 , 115116 (2013)]". Physical Review B 91.3 (2015), p. 039908.

[38] G. Gruner. Density Waves in Solids. Cambridge, MA: Perseus, 1994.

[39] R. Peierls. Quantum Theory of Solids. Oxford: Oxford University Press, 1955.

[40] J. M. Tranquada et al. "Evidence for stripe correlations of spins and holes in copper oxide superconductors". Nature 375.6532 (1995), pp. 561-563.

[41] A. R. Moodenbaugh et al. "Superconducting properties of $\mathrm{La}_{2-x} \mathrm{Ba}_{x} \mathrm{CuO}_{4}$ ". Physical Review B 38.7 (1988), pp. 4596-4600.

[42] P. Abbamonte et al. "Spatially modulated 'Mottness' in $\mathrm{La}_{2-x} \mathrm{Ba}_{x} \mathrm{CuO}_{4}$ ". Nature Physics 1.3 (2005), pp. 155-158.

[43] C. Howald et al. "Periodic density-of-states modulations in superconducting $\mathrm{Bi}_{2} \mathrm{Sr}_{2} \mathrm{CaCu}_{2} \mathrm{O}_{8+\delta}$ ". Physical Review B 67.1 (2003), p. 014533.

[44] W. D. Wise et al. "Charge density wave origin of cuprate checkerboard visualized by scanning tunneling microscopy". Nature Physics 4. (2008), p. 696.

[45] J. E. Hoffman et al. "A four unit cell periodic pattern of quasi-particle states surrounding vortex cores in $\mathrm{Bi}_{2} \mathrm{Sr}_{2} \mathrm{CaCu}_{2} \mathrm{O}_{8+\delta}$ ". Science 295.5554 (2002), pp. 466469.

[46] T. Hanaguri et al. "A 'checkerboard' electronic crystal state in lightly hole-doped Ca2-xNaxCuO2Cl2." Nature 430.August (2004), pp. 1001-1005.

[47] G. Ghiringhelli et al. "Long-Range Incommensurate Charge Fluctuations in (Y,Nd)Ba $\mathrm{Ba}_{2} \mathrm{Cu}_{3} \mathrm{O}_{6+x} "$. Science 337.6096 (2012), pp. 821-825.

[48] E. Blackburn et al. "X-Ray Diffraction Observations of a Charge-Density-Wave Order in Superconducting Ortho-II $\mathrm{YBa}_{2} \mathrm{Cu}_{3} \mathrm{O}_{6.54}$ ". Physical Review Letters 110.13 (2013), p. 137004.

[49] J. Chang et al. "Direct observation of competition between superconductivity and charge density wave order in $\mathrm{YBa}_{2} \mathrm{Cu}_{3} \mathrm{O}_{6.67}$ ". Nature Physics 8.12 (2012), pp. $871-876$.

[50] W. Tabis et al. "Charge order and its connection with Fermi-liquid charge transport in a pristine high- $\mathrm{T}_{c}$ cuprate". Nature Communications 5 (2014), p. 5875. 
[51] N. Doiron-Leyraud et al. "Quantum oscillations and the Fermi surface in an underdoped high- $\mathrm{T}_{c}$ superconductor." Nature 447.7144 (2007), pp. 565-8.

[52] N. Barišić et al. "Universal quantum oscillations in the underdoped cuprate superconductors". Nature Physics 9.12 (2013), pp. 761-764.

[53] S. E. Sebastian, N. Harrison, and G. G. Lonzarich. "Towards resolution of the Fermi surface in underdoped high- $\mathrm{T}_{c}$ superconductors." Reports on progress in Physics 75.10 (2012), p. 102501.

[54] S. Gerber et al. "Three-dimensional charge density wave order in $\mathrm{YBa}_{2} \mathrm{Cu}_{3} \mathrm{O}_{6.67}$ at high magnetic fields". Science 350.6263 (2015), pp. 949-952.

[55] J. Chang et al. "Magnetic field controlled charge density wave coupling in underdoped $\mathrm{YBa}_{2} \mathrm{Cu}_{3} \mathrm{O}_{6+x}$ ". Nature Communications 7.May (2016), p. 11494.

[56] H. Jang et al. "Ideal charge density wave order in the high-field state of superconducting YBCO”. arXiv: (2016), p. 1607.05359.

[57] T. Wu et al. "Magnetic-field-induced charge-stripe order in the high-temperature superconductor $\mathrm{YBa}_{2} \mathrm{Cu}_{3} \mathrm{O}_{y}$." Nature 477.7363 (2011), pp. 191-4.

[58] R. Comin and A. Damascelli. "Resonant x-ray scattering studies of charge order in cuprates". arXiv (2015), pp. 1-26.

[59] M. Vojta. "Lattice symmetry breaking in cuprate superconductors: Stripes, nematics, and superconductivity". Advances in Physics 58.6 (2009), pp. 699-820.

[60] B. J. Ramshaw et al. "Quasiparticle mass enhancement approaching optimal doping in a high-Tc superconductor". Science March (2015), pp. 1-9.

[61] A. I. Larkin and Y. N. Ovchinnikov. "Inhomogeneous state of superconductors". Sov. Phys. JETP 20 (1964), pp. 762-769.

[62] P. Fulde and R. A. Ferrell. "Superconductivity in a Strong Spin-Exchange Field". Physical Review 135.3A (1964), A550-A563.

[63] E. Berg, E. Fradkin, and S. A. Kivelson. "Theory of the striped superconductor". Physical Review B 79.6 (2009), pp. 1-15.

[64] R. Anglani et al. "Crystalline color superconductors". Reviews of Modern Physics 86.2 (2014), pp. 509-561.

[65] G. Binnig and H. Rohrer. "Scanning tunneling microscopy". Surface Science 126.126 (1982), pp. 236-244. 
[66] L. Esaki. "New phenomenon in narrow germanium p-n junctions". Physical Review 109.2 (1958), pp. 603-604.

[67] I. Giaever. "Energy gap in superconductors measured by electron tunneling". Physical Review Letters 5.4 (1960), pp. 147-148.

[68] J. Bardeen. "Tunnelling from a many-particle point of view". Physical Review Letters 6.2 (1961), pp. 6-8.

[69] M. H. Cohen, L. M. Falicov, and J. C. Phillips. "Superconductive Tunneling". Physical Review Letters 8.8 (1962), pp. 316-318.

[70] J. Hoffman. "A Search for Alternative Electronic Order in the by Scanning Tunneling High Temperature Superconductor $\mathrm{Bi}_{2} \mathrm{Sr}_{2} \mathrm{CaCu}_{2} \mathrm{O}_{8+\delta}$ by Scanning Tunneling Microscopy." PhD thesis. University of California, Berkeley, 2003.

[71] W. A. Harrison. "Tunneling from an Independent-Particle Point of View". Physical Review 123.1 (1961), pp. 85-89.

[72] B. Josephson. "Possible new effects in superconductive tunnelling". Physics Letters 1.7 (1962), pp. 251-253.

[73] J. Bardeen. "Tunneling into superconductors". Physical Review Letters 9.4 (1962), pp. 147-149.

[74] M. Tinkham. Introduction to Superconductivity. New York: McGraw-Hill Book Co., 1975 .

[75] Adapted from IAP/TU Wien STM Gallery under Creative Commons Attribution ShareAlike 2.0 Austria License.

[76] J. Tersoff and D. R. Hamann. "Theory of the scanning tunneling microscope". Physical Review B 31.2 (1985), pp. 805-813.

[77] C. J. Chen. Introduction to Scanning Tunneling Microscopy. Oxford University Press, 2007, pp. 1-40.

[78] S. H. Pan, E. W. Hudson, and J. C. Davis. "3He refrigerator based very low temperature scanning tunneling microscope". Review of Scientific Instruments 70.2 (1999), p. 1459.

[79] M. H. Hamidian. "Imaging the Realm of the Strongly Correlated: Visualising Heavy Fermion Formation and The Impact of Kondo Holes in $\mathrm{URu}_{2} \mathrm{Si}_{2} " . \mathrm{PhD}$ thesis. Cornell University, 2011. 
[80] E. W. Hudson. "Investigating High- $\mathrm{T}_{c}$ Superconductivity on the Atomic Scale by Scanning Tunneling Microscopy by" (1994).

[81] C. B. Taylor. "Coexistence of Bogoliubov Quasiparticles and Electronic Cluster Domains in Lightly Hole-Doped Cuprate Superconductors". PhD thesis. Cornell University, 2008, p. 211.

[82] M. Zhiqiang et al. "Relation of the superstructure modulation and extra-oxygen local-structural distortion in Bi”. Physical Review B 55.14 (1997), pp. 9130-9135.

[83] X. B. Kan and S. C. Moss. "Fourdimensional crystallographic analysis of the incommensurate modulation in a $\mathrm{Bi}_{2} \mathrm{Sr}_{2} \mathrm{CaCu}_{2} \mathrm{O}_{8}$ single crystal". Acta Crystallographica Section B 48.2 (1992), pp. 122-134.

[84] J. A. Slezak. "Atomic-Scale Impact of Unit Cell Dimensions on Pairing in a High-Temperature Superconductor". PhD thesis. Cornell University, 2007.

[85] Y. Kohsaka et al. "Growth of Na-doped $\mathrm{Ca}_{2} \mathrm{CuO}_{2} \mathrm{Cl}_{2}$ single crystals under high pressures of several GPa". Journal of the American Chemical Society 124.41 (2002), pp. $12275-12278$.

[86] M. F. Crommie, C. P. Lutz, and D. M. Eigler. "Imaging standing waves in a two-dimensional electron gas". Nature 363 (1993), p. 524.

[87] Q.-H. Wang and D.-H. Lee. "Quasiparticle scattering interference in hightemperature superconductors". Physical Review B 67.2 (2003), p. 020511.

[88] J. A. Slezak et al. "Imaging the impact on cuprate superconductivity of varying the interatomic distances within individual crystal unit cells." Proceedings of the National Academy of Sciences of the United States of America 105.9 (2008), pp. $3203-8$.

[89] M. H. Hamidian et al. "Picometer registration of zinc impurity states in $\mathrm{Bi}_{2} \mathrm{Sr}_{2}$ $\mathrm{CaCu}_{2} \mathrm{O}_{8+\delta}$ for phase determination in intra-unit-cell Fourier transform STM". New Journal of Physics 14.5 (2012), p. 053017.

[90] J.-X. Li, C.-Q. Wu, and D.-H. Lee. "Checkerboard charge density wave and pseudogap of high-Tc cuprate". Physical Review B 74.18 (2006), p. 184515.

[91] K. Seo, H.-D. Chen, and J. Hu. "D-Wave Checkerboard Order in Cuprates". Physical Review B 76.2 (2007), p. 020511. 
[92] D. M. Newns and C. C. Tsuei. "Fluctuating $\mathrm{CuOCu}$ bond model of hightemperature superconductivity". Nature Physics 3.3 (2007), pp. 184-191.

[93] C. Honerkamp, H. C. Fu, and D.-H. Lee. "Phonons and d-wave pairing in the two-dimensional Hubbard model". Physical Review B 75.1 (2007), p. 014503.

[94] M. Metlitski and S. Sachdev. "Instabilities near the onset of spin density wave order in metals". New Journal of Physics 12.10 (2010), p. 105007.

[95] Y. Wang and A. Chubukov. "Charge-density-wave order with momentum $(2 \mathrm{Q}, 0)$ and $(0,2 \mathrm{Q})$ within the spin-fermion model: Continuous and discrete symmetry breaking, preemptive composite order, and relation to pseudogap in hole-doped cuprates". Physical Review B 90.3 (2014), pp. 1-52.

[96] T. Holder and W. Metzner. "Incommensurate nematic fluctuations in twodimensional metals". Physical Review B 85.16 (2012), p. 165130.

[97] K. B. Efetov, H. Meier, and C. Pépin. "Pseudogap state near a quantum critical point". Nature Physics 9.7 (2013), pp. 442-446.

[98] S. Bulut, W. A. Atkinson, and A. P. Kampf. "Spatially modulated electronic nematicity in the three-band model of cuprate superconductors". Physical Review B 88.15 (2013), p. 155132.

[99] S. Sachdev and R. La Placa. "Bond Order in Two-Dimensional Metals with Antiferromagnetic Exchange Interactions". Physical Review Letters 111.2 (2013), p. 027202 .

[100] J. C. S. Davis and D.-H. Lee. "Concepts relating magnetic interactions, intertwined electronic orders, and strongly correlated superconductivity." Proceedings of the National Academy of Sciences of the United States of America (2013), pp. $1-8$.

[101] A. Allais, J. Bauer, and S. Sachdev. "Density wave instabilities in a correlated two-dimensional metal". Physical Review B 90.15 (2014), pp. 1-5.

[102] M. H. Fischer et al. "Nematic and spin-charge orders driven by hole-doping a charge-transfer insulator". New Journal of Physics 16 (2014).

[103] C. Pépin et al. "Pseudogap, charge order, and pairing density wave at the hot spots in cuprate superconductors". Physical Review B 90.19 (2014), pp. 1-16. 
[104] D. Chowdhury and S. Sachdev. "Density-wave instabilities of fractionalized Fermi liquids". Physical Review B 90.24 (2014), pp. 1-10.

[105] W. A. Atkinson, A. P. Kampf, and S. Bulut. "Charge order in the pseudogap phase of cuprate superconductors". New Journal of Physics 17.1 (2015), p. 13025.

[106] Y. Yamakawa and H. Kontani. "Spin-fluctuation-driven nematic charge-density wave in cuprate superconductors: Impact of aslamazov-larkin vertex corrections". Physical Review Letters 114.25 (2015), pp. 1-6.

[107] P. Monthoux. " $\mathrm{d}_{x^{2}-y^{2}}$ Pairing and Spin Fluctuations in the Cuprate Superconductors: A Progress". 56.12 (2003), pp. 1-8.

[108] T. P. Devereaux et al. "Anisotropic electron-phonon interaction in the cuprates". Physical Review Letters 93.11 (2004), pp. 1-4.

[109] M. Le Tacon et al. "Inelastic X-ray scattering in $\mathrm{YBa}_{2} \mathrm{Cu}_{3} \mathrm{O}_{6.6}$ reveals giant phonon anomalies and elastic central peak due to charge-density-wave formation". Nature Physics 10.1 (2013), pp. 52-58.

[110] V. Mishra and M. R. Norman. "Strong coupling critique of spin fluctuation driven charge order in underdoped cuprates". Physical Review B 92.6 (2015), pp. 1-4.

[111] M. Vojta. "Lattice symmetry breaking in cuprate superconductors: stripes, nematics, and superconductivity". Advances in Physics 58.6 (2009), pp. 699-820.

[112] Y Kohsaka et al. "How Cooper pairs vanish approaching the Mott insulator in $\mathrm{Bi}_{2} \mathrm{Sr}_{2} \mathrm{CaCu}_{2} \mathrm{O}_{8+\delta}$." Nature 454.7208 (2008), pp. 1072-8.

[113] Y. Kohsaka et al. "Visualization of the emergence of the pseudogap state and the evolution to superconductivity in a lightly hole-doped Mott insulator". Nature Physics 8.7 (2012), pp. 534-538.

[114] R. Comin et al. "Symmetry of charge order in cuprates". Nature Materials 14.8 (2015), pp. 796-800.

[115] E. M. Forgan et al. "The microscopic structure of charge density waves in underdoped $\mathrm{YBa}_{2} \mathrm{Cu}_{3} \mathrm{O}_{6.54}$ revealed by X-ray diffraction." Nature communications 6 (2015), p. 10064.

[116] A. J. Achkar et al. "Orbital symmetry of charge-density-wave order in $\mathrm{La}_{1.875} \mathrm{Ba}_{0.125} \mathrm{CuO}_{4}$ and $\mathrm{YBa}_{2} \mathrm{Cu}_{3} \mathrm{O}_{6.67}$ ". Nature Materials 15.6 (2016), pp. 616620. 
[117] M. A. Metlitski and S. Sachdev. "Quantum phase transitions of metals in two spatial dimensions. II. Spin density wave order". Physical Review B 82.7 (2010), p. 075128.

[118] K. Lee, S. A. Kivelson, and E.-A. Kim. "Cold-spots and glassy nematicity in underdoped cuprates". 014204 (2016), pp. 1-9.

[119] E. H. Da Silva Neto et al. "Ubiquitous Interplay between Charge Ordering and High-Temperature Superconductivity in Cuprates." Science 393 (2013).

[120] S. Blanco-Canosa et al. "Momentum-dependent charge correlations in $\mathrm{YBa}_{2} \mathrm{Cu}_{3} \mathrm{O}_{6+\delta}$ superconductors probed by resonant x-ray scattering: Evidence for three competing phases". Physical Review Letters 110.18 (2013), pp. 1-5.

[121] R. Comin et al. "Charge Order Driven by Fermi-Arc Instability in $\mathrm{Bi}_{2} \mathrm{Sr}_{2-x} \mathrm{La}_{x} \mathrm{CuO}_{6+\delta}$ ". Science 343. (2014), pp. 390-392.

[122] L. Nie, G. Tarjus, and S. a. Kivelson. "Quenched disorder and vestigial nematicity in the pseudogap regime of the cuprates". Proceedings of the National Academy of Sciences 111.22 (2014), pp. 7980-7985.

[123] A. J. Achkar et al. "Impact of quenched oxygen disorder on charge density wave order in $\mathrm{YBa}_{2} \mathrm{Cu}_{3} \mathrm{O}_{6+x}$ ". Physical Review Letters 113.10 (2014), pp. 1-5.

[124] J. Chang et al. "Direct observation of competition between superconductivity and charge density wave order in $\mathrm{YBa}_{2} \mathrm{Cu}_{3} \mathrm{O}_{6.67}$ ". Nature Physics 8.12 (2012), pp. $871-876$.

[125] G. Ghiringhelli et al. "Long-range incommensurate charge fluctuations in (Y,Nd)Ba $\mathrm{Cu}_{3} \mathrm{O}_{6+x} . "$ Science 337.6096 (2012), pp. 821-5.

[126] R. Comin et al. "Broken translational and rotational symmetry via charge stripe order in underdoped $\mathrm{YBa}_{2} \mathrm{Cu}_{3} \mathrm{O}_{6+y}$ ". Science 347.6228 (2015), pp. 1335-1339.

[127] J. Robertson et al. "Distinguishing patterns of charge order: Stripes or checkerboards". Physical Review B 74.13 (2006), p. 134507.

[128] P. Corboz et al. "Stripes in the two-dimensional t-J model with infinite projected entangled-pair states". Physical Review B 84.4 (2011), pp. 1-5.

[129] B. Burk et al. "Charge-density-wave domains in $1 \mathrm{~T}-\mathrm{TaS}_{2}$ observed by satellite structure in scanning-tunneling-microscopy images". Physical Review Letters 66.23 (1991), pp. 3040-3043. 
[130] A. Mesaros et al. "Commensurate $4 \mathrm{a}_{0}$ period Charge Density Modulations throughout the $\mathrm{Bi}_{2} \mathrm{Sr}_{2} \mathrm{CaCu}_{2} \mathrm{O}_{8+x}$ Pseudogap Regime" (2016).

[131] E. H. da Silva Neto et al. "Charge ordering in the electron-doped superconductor $\mathrm{Nd}_{2-x} \mathrm{Ce}_{x} \mathrm{CuO}_{4}$ ". Science 347.6219 (2015), pp. 282-285.

[132] M. H. Hamidian et al. "Detection of a Cooper-pair density wave in $\mathrm{Bi}_{2} \mathrm{Sr}_{2} \mathrm{CaCu}_{2} \mathrm{O}_{8+x}$ ". Nature 532.7599 (2016), pp. 343-347.

[133] J. Šmakov, I. Martin, and A. V. Balatsky. "Josephson scanning tunneling microscopy". Physical Review B 64.21 (2001), p. 212506.

[134] A. Yazdani et al. "Probing the Local Effects of Magnetic Impurities on Superconductivity". Science 275.5307 (1997), pp. 1767-1770.

[135] M. I. Salkola, A. V. Balatsky, and J. R. Schrieffer. "Spectral Properties of Quasiparticle Excitations Induced by Magnetic Moments in Superconductors". Physical Review B 55.18 (1997), p. 12648.

[136] S. H. Ji et al. "High-resolution scanning tunneling spectroscopy of magnetic impurity induced bound states in the superconducting gap of $\mathrm{Pb}$ thin films". Physical Review Letters 100.22 (2008), pp. 16-18.

[137] K. J. Franke, G. Schulze, and J. I. Pascual. "Competition of Superconducting Phenomena and Kondo Screening at the Nanoscale". Science 332.6032 (2011), pp. 940-944.

[138] S. H. Pan et al. "STM Studies of the Electronic Structure of Vortex Cores in $\mathrm{Bi}_{2} \mathrm{Sr}_{2} \mathrm{CaCu}_{2} \mathrm{O}_{8+\delta}$ ". Physical Review Letters 85.7 (2000), pp. 1536-1539.

[139] O. Naaman, W Teizer, and R. C. Dynes. "Fluctuation dominated Josephson tunneling with a scanning tunneling microscope." Physical Review Letters 87.9 (2001), p. 097004.

[140] J. G. Rodrigo, H. Suderow, and S. Vieira. "On the use of STM superconducting tips at very low temperatures". European Physical Journal B 40.4 (2004), pp. $483-488$.

[141] T. Proslier et al. "Probing the superconducting condensate on a nanometer scale". Europhysics Letters 962 (2006), p. 7.

[142] A. Roychowdhury et al. "Microwave photon-assisted incoherent cooper-pair tunneling in a Josephson STM". Physical Review Applied 4.3 (2015), pp. 1-7. 
[143] B. Jäck et al. "A nanoscale gigahertz source realized with Josephson scanning tunneling microscopy". Applied Physics Letters 106.1 (2015), p. 013109.

[144] B. Jäck et al. "Critical Josephson current in the dynamical Coulomb blockade regime". Physical Review B 93.2 (2016), p. 020504.

[145] M. T. Randeria et al. "Scanning Josephson spectroscopy on the atomic scale" (2016).

[146] P. W. Anderson and J. M. Rowell. "Probable observation of the Josephson superconducting tunneling effect". Physical Review Letters 10.6 (1963), pp. 230232.

[147] P. W. Anderson. Special Effects in Superconductivity. Academic Press Inc., 1964, pp. 113-135.

[148] L. P. Gor'kov. Microscopic Derivation of the Ginzburg-Landau Equations in the Theory of Superconductivity. 1959.

[149] W. C. Stewart. "Current-voltage characteristics of Josephson junctions". Applied Physics Letters 12.8 (1968), pp. 277-280.

[150] D. E. McCumber. "Effect of ac impedance on dc voltage-current characteristics of superconductor weak-link junctions". Journal of Applied Physics 39.7 (1968), pp. 3113-3118.

[151] V. Ambegaokar and B. I. Halperin. "Voltage due to thermal noise in the dc Josephson effect". Physical Review Letters 22.25 (1969), pp. 1364-1366.

[152] Y. M. Ivanchenko and L. A. Zil 'berman. "The Josephson Effect in Small Tunnel Contacts". Soviet Physics JETP 28.6 (1969), pp. 1272-1276.

[153] R. L. Kautz and J. M. Martinis. "Noise-affected I - V curves in small hysteretic Josephson junctions". Physical Review B 42.16 (1990), pp. 9903-9937.

[154] J. M. Martinis and R. L. Kautz. "Classical phase diffusion in small hysteretic Josephson junctions". Physical Review Letters 63.14 (1989), pp. 1507-1510.

[155] H. Grabert and M. H. Devoret. Single Charge Tunneling. Ed. by H. Grabert and M. H. Devoret. Vol. 294. NATO ASI Series. Boston, MA: Springer US, 1992.

[156] A. N. Cleland, J. M. Schmidt, and J. Clarke. "Charge fluctuations in smallcapacitance junctions". Physical Review Letters 64.13 (1990), pp. 1565-1568. 
[157] M. Devoret et al. "Effect of the electromagnetic environment on the Coulomb blockade in ultrasmall tunnel junctions". Physical review ... 65.15 (1990), pp. 2015-2018.

[158] G. Falci, V. Bubanja, and G. Schön. "Quantum tunnelling in small-capacitance Josephson junctions in a general electromagnetic environment". EPL (Europhysics Letters) 109 (1991).

[159] G. Falci, V. Bubanja, and G. Schon. "Quasiparticle and Cooper pair tunneling in small capacitance Josephson junctions". Z.Phys.B-Condensed Matter 85 (1991), pp. $451-458$.

[160] G. L. Ingold and H Grabert. "Finite-Temperature Current-Voltage Characteristics of Ultrasmall Tunnel Junctions". Europhysics Letters (EPL) 14.4 (1991), pp. $371-376$.

[161] G. L. Ingold, H. Grabert, and U. Eberhardt. "Cooper-pair current through ultrasmall Josephson junctions". Physical Review B 50.1 (1994), pp. 395-402.

[162] A. O. Caldeira and A. J. Leggett. "Quantum tunneling in a dissipative system". Annals of Physics 149 (1983), pp. 374-456.

[163] B. Nachumi et al. "Muon spin relaxation studies of Zn-substitution effects in high-T ${ }_{c}$ cuprate superconductors". Physical Review Letters 77 (1996), pp. 54215424.

[164] S. S. Pan et al. "Imaging the effects of individual zinc impurity atoms on superconductivity in $\mathrm{Bi}_{2} \mathrm{Sr}_{2} \mathrm{CaCu}_{2} \mathrm{O}_{8+\delta}$ ". Nature 403.6771 (2000), pp. 746-50.

[165] H. Kimura et al. "Josephson scanning tunneling microscopy: A local and direct probe of the superconducting order parameter". Physical Review B 80.14 (2009), pp. $1-16$.

[166] A. G. Sun et al. "Observation of Josephson pair tunneling between a high- $\mathrm{T}_{c}$ cuprate $\left(\mathrm{YBa}_{2} \mathrm{Cu}_{3} \mathrm{O}_{7-\delta}\right)$ and a conventional superconductor $(\mathrm{Pb})$ ". Physical Review Letters 72.14 (1994), pp. 2267-2270.

[167] D. F. Agterberg and H. Tsunetsugu. "Dislocations and vortices in pair density wave superconductors". Nature Physics 4.8 (2009), p. 6.

[168] E. Fradkin, S. A. Kivelson, and J. M. Tranquada. "Colloquium : Theory of intertwined orders in high temperature superconductors". Reviews of Modern Physics 87.2 (2015), pp. 457-482. 
[169] A. M. Clogston. "Upper limit for the critical field in hard superconductors". Physical Review Letters 9.6 (1962), pp. 266-267.

[170] Y. Matsuda and H. Shimahara. "Fulde-Ferrell-Larkin-Ovchinnikov state in heavy fermion superconductors". Journal of the Physical Society of Japan 76.5 (2007), pp. $1-16$.

[171] D. Saint-James. Type II Superconducivity. New York, NY: Pergamon Press, 1969.

[172] Y.-A. Liao et al. "Spin-imbalance in a one-dimensional Fermi gas." Nature 467.7315 (2010), pp. 567-9.

[173] Q. Li et al. "Two-dimensional superconducting fluctuations in stripe-ordered La1.875Ba0.125CuO4". Physical Review Letters 99.6 (2007), pp. 4-7.

[174] M. Hücker et al. "Stripe order in superconducting $\mathrm{La}_{2-x} \mathrm{Ba}_{x} \mathrm{CuO}_{4}(0.095 \leq x \leq$ 0.155)". Physical Review B 83.10 (2011), p. 104506.

[175] S. Tajima et al. "c-Axis optical response in the static stripe ordered phase of the cuprates". Physical Review Letters 86.3 (2001), pp. 500-503.

[176] J. Orenstein. "Josephson plasmons: The new wave". Nature Physics 2.8 (2006), pp. 503-504.

[177] A. A. Schafgans et al. "Towards a two-dimensional superconducting state of $\mathrm{La}_{2-x} \mathrm{Sr}_{x} \mathrm{CuO}_{4}$ in a moderate external magnetic field". Physical Review Letters 104.15 (2010), pp. 1-4.

[178] A. Himeda, T. Kato, and M. Ogata. "Stripe States with Spatially Oscillating d-Wave Superconductivity in the Two-Dimensional $t-t^{\prime}-J$ Model". Physical Review Letters 88.11 (2002), p. 117001.

[179] E. Berg et al. "Dynamical layer decoupling in a stripe-ordered high- $\mathrm{T}_{c}$ superconductor". Physical Review Letters 99.12 (2007), pp. 1-4.

[180] M. K. Crawford et al. "Lattice instabilities and the effect of copper-oxygen-sheet distortions on superconductivity in doped $\mathrm{La}_{2} \mathrm{CuO}_{4}$ ". Physical Review B 44.14 (1991), pp. 7749-7752.

[181] J. D. Axe et al. "Structural phase transformations and superconductivity in $\mathrm{La}_{2-x} \mathrm{Ba}_{x} \mathrm{CuO}_{4} "$. Physical Review Letters 62.23 (1989), pp. 2751-2754. 
[182] A. Dubroka et al. "Evidence of a precursor superconducting phase at temperatures as high as $180 \mathrm{~K}$ in $\mathrm{RBa}_{2} \mathrm{Cu}_{3} \mathrm{O}_{7-\delta}(\mathrm{R}=\mathrm{Y}, \mathrm{Gd}, \mathrm{Eu})$ superconducting crystals from infrared spectroscopy". Physical Review Letters 106.4 (2011), pp. 1-4.

[183] F. Yu et al. "Diamagnetic response in under-doped $\mathrm{YBa}_{2} \mathrm{Cu}_{3} \mathrm{O}_{6.6}$ in high magnetic fields". arXiv: 1402.7371 (2014),

[184] P. A. Lee. "Amperean pairing and the pseudogap phase of cuprate superconductors". Physical Review X 4.3 (2014), pp. 1-13.

[185] R.-H. He et al. "From a Single-Band Metal to a High-Temperature Superconductor via Two Thermal Phase Transitions". Science 331.6024 (2011), pp. 15791583.

[186] H.-B. Yang et al. "Reconstructed Fermi Surface of Underdoped $\mathrm{Bi}_{2} \mathrm{Sr}_{2} \mathrm{CaCu}_{2} \mathrm{O}_{8+\delta}$ ". Physical Review Letters 107.4 (2011), p. 047003.

[187] T. Kondo et al. "Disentangling Cooper-pair formation above the transition temperature from the pseudogap state in the cuprates". Nature Physics 7.1 (2010), pp. 21-25.

[188] J. H. Ma et al. "Coexistence of competing orders with two energy gaps in real and momentum space in the high temperature superconductor $\mathrm{Bi}_{2} \mathrm{Sr}_{2-x} \mathrm{La}_{x} \mathrm{CuO}_{6+\delta}$ ". Physical Review Letters 101.20 (2008), pp. 1-4.

[189] J. Meng et al. "Monotonic d-wave superconducting gap of the optimally doped $\mathrm{Bi}_{2} \mathrm{Sr}_{1.6} \mathrm{La}_{0.4} \mathrm{CuO}_{6}$ superconductor by laser-based angle-resolved photoemission spectroscopy". Physical Review B 79.2 (2009), pp. 2-5.

[190] K. Nakayama et al. "Evolution of a pairing-induced pseudogap from the superconducting gap of $(\mathrm{Bi}, \mathrm{Pb})_{2} \mathrm{Sr}_{2} \mathrm{CuO}_{6}$ ". Physical Review Letters 102.22 (2009), pp. 1316.

[191] R. M. Fernandes, a. V. Chubukov, and J. Schmalian. "What drives nematic order in iron-based superconductors?" Nature Physics 10.2 (2014), pp. 97-104.

[192] L. Nie, G. Tarjus, and S. a. Kivelson. "Quenched disorder and vestigial nematicity in the pseudogap regime of the cuprates". Proceedings of the National Academy of Sciences 111.22 (2014), pp. 7980-7985.

[193] S. A. Kivelson, E. Fradkin, and V. J. Emery. "Electronic liquid-crystal phases of a doped Mott insulator". Nature 393.6685 (1998), pp. 550-553. 
[194] P. A. Lee, N. Nagaosa, and X. G. Wen. "Doping a Mott insulator: Physics of high-temperature superconductivity". Reviews of Modern Physics 78.1 (2006).

[195] Y. Wang, D. F. Agterberg, and A. Chubukov. "Coexistence of Charge-DensityWave and Pair-Density-Wave Orders in Underdoped Cuprates". Physical Review Letters 114.19 (2015), pp. 1-6.

[196] P. Corboz, T. M. Rice, and M. Troyer. "Competing states in the t - J model: Uniform d -wave state versus stripe state". Physical Review Letters 113.4 (2014), pp. $1-5$.

[197] F. Loder et al. "Mean-field pairing theory for the charge-stripe phase of hightemperature cuprate superconductors". Physical Review Letters 107.18 (2011), pp. $1-4$.

[198] F. Loder, A. P. Kampf, and T. Kopp. "Superconducting state with a finitemomentum pairing mechanism in zero external magnetic field". Physical Review B 81.2 (2010), pp. 1-4.

[199] M. Raczkowski et al. "Unidirectional d -wave superconducting domains in the two-dimensional t-J model". Physical Review B 76.14 (2007), pp. 3-6.

[200] R. Soto-Garrido and E. Fradkin. "Pair-density-wave superconducting states and electronic liquid-crystal phases". Physical Review B 89.16 (2014), pp. 1-19.

[201] M. Zelli, C. Kallin, and A. J. Berlinsky. "Quantum oscillations in a $\pi$-striped superconductor". Physical Review B 86.10 (2012), pp. 1-16.

[202] K. Y. Yang et al. "Nature of stripes in the generalized t-J model applied to the cuprate superconductors". New Journal of Physics 11 (2009).

[203] P. W. Anderson. "Resonating valence bonds: A new kind of insulator?" Materials Research Bulletin 8.2 (1973), pp. 153-160.

[204] G. Kotliar and J. Liu. "Superexchange mechanism and d -wave superconductivity". Physical Review B 38.7 (1988), pp. 5142-5145.

[205] S. S. Lee, P. A. Lee, and T. Senthil. "Amperean pairing instability in the U(1) spin liquid state with fermi surface and application to $\kappa$-(BEDT-TTF $)_{2} \mathrm{Cu}_{2}(\mathrm{CN})_{3}$ ". Physical Review Letters 98.6 (2007), pp. 1-4.

[206] D. Chowdhury and S. Sachdev. "Feedback of superconducting fluctuations on charge order in the underdoped cuprates". Physical Review B 90.13 (2014). 
[207] Y. Wang, D. F. Agterberg, and A. Chubukov. "Interplay between pair- and charge-density-wave orders in underdoped cuprates". Physical Review B 91.11 (2015), p. 115103.

[208] K. Seo, H.-D. Chen, and J. Hu. "Complementary pair-density-wave and d-wavecheckerboard orderings in high-temperature superconductors". Physical Review B 78.9 (2008), p. 094510.

[209] E. Berg, E. Fradkin, and S. A. Kivelson. "Charge-4e superconductivity from pair density wave order in certain high temperature superconductors". Nature Physics 5.11 (2009), p. 5.

[210] H.-D. Chen et al. "Pair density wave in the pseudogap state of high temperature superconductors." Physical Review Letters 93.18 (2004), p. 187002.

[211] H. Freire, V. S. de Carvalho, and C. Pépin. "Renormalization group analysis of the pair-density-wave and charge order within the fermionic hot-spot model for cuprate superconductors". Physical Review B 92.4 (2015), p. 045132. 